\title{
Advanced Algorithms for Modeling and Simulation of High-Speed Interconnects
}

\author{
by \\ Changzhong Chen
}

\author{
A thesis submitted to the \\ Faculty of Graduate Studies and Research \\ in partial fulfillment of the requirements \\ for the degree of \\ Doctor of Philosophy
}
Ottawa-Carleton Institute for Electrical and Computer Engineering
Department of Electronics
Faculty of Engineering
Carleton University
Ottawa, Ontario, Canada

September, 2007

(C)Changzhong Chen, 2007 


$\begin{array}{ll}\begin{array}{l}\text { Library and } \\ \text { Archives Canada }\end{array} & \begin{array}{l}\text { Bibliothèque et } \\ \text { Archives Canada }\end{array} \\ \begin{array}{l}\text { Published Heritage } \\ \text { Branch }\end{array} & \begin{array}{l}\text { Direction du } \\ \text { Patrimoine de l'édition }\end{array} \\ \begin{array}{l}\text { 395 Wellington Street } \\ \text { Ottawa ON K1A ON4 }\end{array} & \begin{array}{l}\text { 395, rue Wellington } \\ \text { Ottawa ON K1A ON4 } \\ \text { Canada }\end{array}\end{array}$

Your file Votre référence ISBN: 978-0-494-33485-0 Our file Notre référence ISBN: 978-0-494-33485-0

NOTICE:

The author has granted a nonexclusive license allowing Library and Archives Canada to reproduce, publish, archive, preserve, conserve, communicate to the public by telecommunication or on the Internet, loan, distribute and sell theses worldwide, for commercial or noncommercial purposes, in microform, paper, electronic and/or any other formats.

The author retains copyright ownership and moral rights in this thesis. Neither the thesis nor substantial extracts from it may be printed or otherwise reproduced without the author's permission.
AVIS:

L'auteur a accordé une licence non exclusive permettant à la Bibliothèque et Archives Canada de reproduire, publier, archiver, sauvegarder, conserver, transmettre au public par télécommunication ou par l'Internet, prêter, distribuer et vendre des thèses partout dans le monde, à des fins commerciales ou autres, sur support microforme, papier, électronique et/ou autres formats.

L'auteur conserve la propriété du droit d'auteur et des droits moraux qui protège cette thèse. $\mathrm{Ni}$ la thèse ni des extraits substantiels de celle-ci ne doivent être imprimés ou autrement reproduits sans son autorisation.
In compliance with the Canadian

Privacy Act some supporting forms may have been removed from this thesis.

While these forms may be included in the document page count, their removal does not represent any loss of content from the thesis.
Conformément à la loi canadienne sur la protection de la vie privée, quelques formulaires secondaires ont été enlevés de cette thèse.

Bien que ces formulaires aient inclus dans la pagination, il n'y aura aucun contenu manquant.

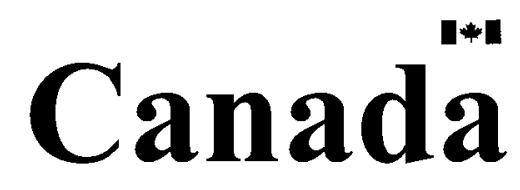




\section{Abstract}

The increasing use of higher operating frequencies has highlighted the previously negligible interconnect effects, such as ringing, delay and crosstalk. Accurate and efficient macromodeling of high-speed interconnect to incorporate these effects has placed new demands on computer-aided-design tools to provide reliable assessment of signal integrity of the whole system. In the meanwhile, due to the skin, edge and proximity effects etc., the perunit-length parameters used to describe the interconnects become frequency-dependent. This puts more strict requirements on the macromodeling techniques.

Passivity and the order of the macromodel are the two key concerns in the macromodeling and simulation of high-speed interconnect networks. Passive macromodels are important to ensure the stability of the overall system. Low order models are desired for efficient simulations. This thesis is intended to provide a systematic way in constructing a passive and reduced-order model for the high-speed interconnect network. To achieve this goal, the following issues are addressed.

First, a novel theory is developed to verify the passivity of method of characteristic based macromodels. This new theory can be employed to those systems described by general algebraic delay differential equations. For those non-passive method of characteristics models, a fast algorithm is also developed to correct the passivity violations, such that the resulting models are guaranteed to be passive. Next, a guaranteed passive macromodeling 
technique based on integrated congruence transform is developed for a general frequencydependent transmission line. The proposed technique provides the advantage that a compact order model can be obtained directly from the Telegrapher's equations without having to model the transmission line using a large lumped network as an intermediate step. Finally, a general model order reduction scheme is developed to reduce a network, including large equivalent circuits with lumped components and delay elements, into a passive and compact model. The resulting model facilitates the analysis of the signal integrity for the entire network. 


\section{Dedication}

To my parents and my wife. 


\section{Acknowledgments}

First, and most importantly, I should thank my supervisors, Prof. Michel Nakhla, Prof. Ram Achar and Prof. Emad Gad, for their relentless support for my work, both intellectually and financially. I am grateful for their helpful comments, suggestions and constructive criticism throughout the entire project. My thesis has benefited substantially from their insightful recommendations. Their suggestions helped me manage difficult problems. Without their support, my thesis would not have been possible.

Special thanks are extended to Prof. Emad Gad for his extensive help in writing the papers, and to Dharmendra Saraswat for the valuable discussions, helpful comments and ideas.

Next, I would like to thank my colleagues for making my research at Carleton an enjoyable experience: Dharmendra Saraswat, Arumika Jerome, Natelie Nakhla, Subhendu Roy, Nigel Rodrigues, Wenliang Tseng, Grace (Zhou) Wang, Yinghong Zhou, Gurpreet Singh and Praveen Pai. I am also grateful for the friendly manner from the office staff, professors and students at Carleton University.

I would like to express my sincere gratitude toward my wife, Li Pan, for her loving support, encouragement, patience and understanding. Her support has helped me a lot in overcoming many difficulties and completing the thesis.

Finally, I leave a special note to my parents, Ziyu and Zhaojuan, and my brothers, Changguo and Changdeng. I am especially grateful to them for many years of patient backing on all things. 


\section{Contents}

Abstract

Acknowledgments

1 Introduction 1

1.1 Background and motivation . . . . . . . . . . . . . 1

1.2 Objectives and contributions .................. 6

1.3 Organization ..................... 8

2 Review of Macromodeling and MOR Techniques 9

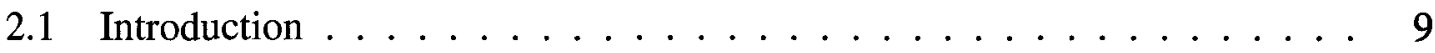

2.2 Passivity conditions . . . . . . . . . . . . . . 11

2.3 Lumped element based models . . . . . . . . . . . . . . . . 13

2.4 Delay extraction based models $\ldots \ldots \ldots \ldots$

2.5 Passivity enforcement . . . . . . . . . . . . . . . . 19

2.6 Model-order reduction $\ldots \ldots \ldots \ldots \ldots \ldots$

2.7 Integrated congruence transform $\ldots \ldots \ldots \ldots$

3 Passivity Verification in Delay-Based Macromodels of Two-Conductor Trans$\begin{array}{ll}\text { mission Lines } & 27\end{array}$

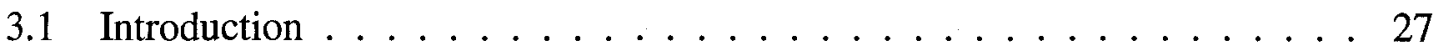

vii 
3.2 Passivity of MoC-based macromodels . . . . . . . . . . . . . . 29

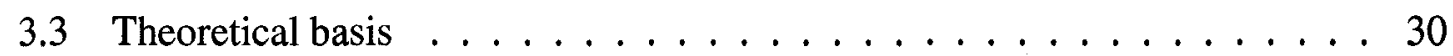

3.3.1 Formulation of ADDE's . . . . . . . . . . . . 32

3.3.2 Passivity verification in MoC macromodels . . . . . . . . . 33

3.3.3 Regions of passivity violation . . . . . . . . . . 36

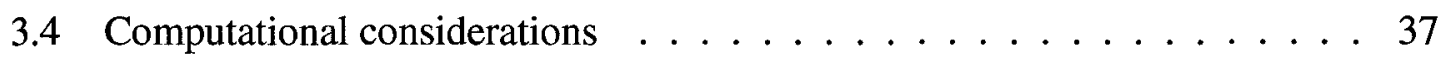

3.4.1 Phase I: Estimation of $\Re\left[\sigma_{k}(s)\right] \ldots \ldots . \ldots \ldots$

3.4.2 Phase II: Locating the PR-ness violations . . . . . . . . . . . . 39

3.5 Computational complexity . . . . . . . . . . . . . 42

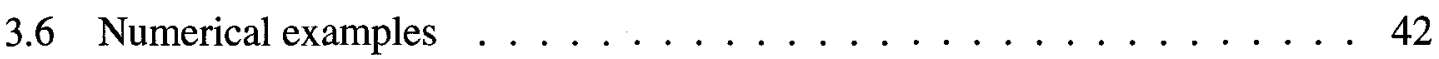

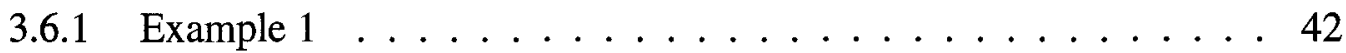

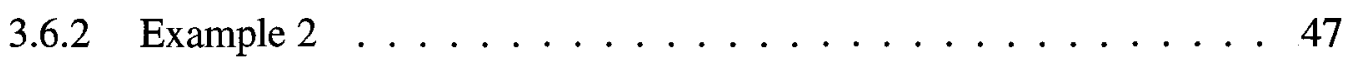

3.6 .3 Example $3 \ldots \ldots \ldots \ldots \ldots \ldots$

4 Passivity Verification in Delay-Based Macromodels of Multi-conductor Transmission Lines

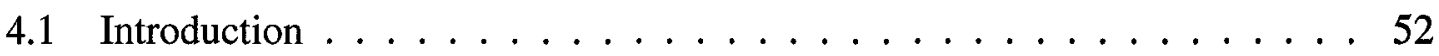

4.2 Passivity of the MoC-based macromodels $\ldots \ldots \ldots \ldots$

4.3 Theoretical basis ............................. 54

4.3.1 Decomposition of MoC macromodels . . . . . . . . . . . . 54

4.3.2 Realization of $\boldsymbol{Y}_{1}(s)$ and $\boldsymbol{Y}_{2}(s) \ldots \ldots \ldots$

4.3.3 Non-negative definiteness of $\boldsymbol{Y}_{1}(s)$ and $\boldsymbol{Y}_{2}(s) \ldots \ldots 7$

4.3.4 Regions of passivity violation ............... 61

4.4 Computational considerations .................... 62

4.5 Numerical examples ..................... 66

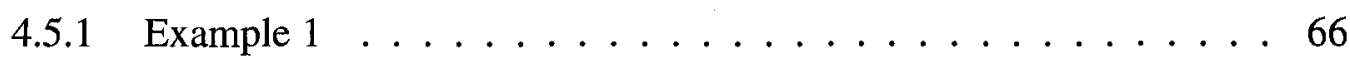

viii 
4.5 .2 Example $2 \ldots \ldots \ldots \ldots \ldots \ldots$

4.5 .3 Example $3 \ldots \ldots \ldots \ldots$. . . . . . . . . . . 69

5 Passivity Enforcement for Method of Characteristics Based Macromodels 72

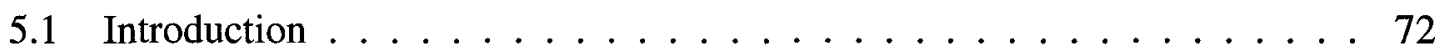

5.2 Proposed passivity enforcement algorithm $\ldots \ldots \ldots \ldots \ldots$

5.2.1 Determine the maximum passivity violation point in a PVR $\ldots 73$

5.2.2 Proposed passivity enforcement $\ldots \ldots \ldots \ldots \ldots$. . . . 74

5.3 Computational complexity . . . . . . . . . . . . . . 79

5.4 Numerical examples $\ldots \ldots \ldots \ldots \ldots \ldots \ldots$

5.4 .1 Example $1 \ldots \ldots \ldots \ldots \ldots \ldots \ldots \ldots \ldots \ldots \ldots \ldots$

5.4 .2 Example $2 \ldots \ldots \ldots \ldots \ldots \ldots \ldots \ldots$

5.4 .3 Example $3 \ldots \ldots \ldots \ldots \ldots$

6 Circuit Modeling for FD-PUL Parameters 91

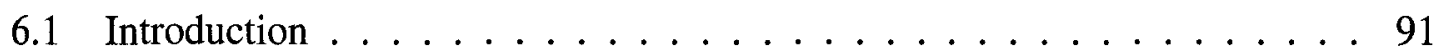

6.2 Development of the proposed modeling algorithm . . . . . . . . 95

6.2.1 Formulation of the approximation $\ldots \ldots \ldots \ldots \ldots$

6.2.2 Error criteria and positive-realness . . . . . . . . . . . 96

6.3 Network synthesis . . . . . . . . . . . . . . . . . . . 97

6.3.1 Realization of the PUL networks using lumped components $\ldots 97$

6.3.2 Realization of the PUL networks using Laplace elements . . . . . 102

6.4 Numerical examples $\ldots \ldots \ldots \ldots \ldots \ldots$. . . . . . . . . . 104

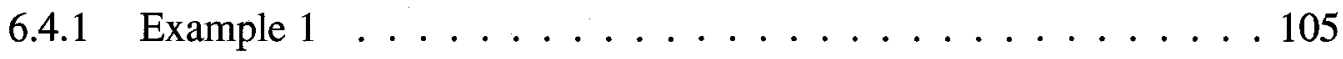

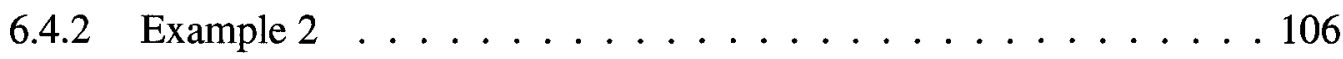

6.4 .3 Example $3 \ldots \ldots \ldots \ldots \ldots \ldots$ 
7 Analysis of Frequency-Dependent Interconnects Using Integrated Congruence

Transform

7.1 Introduction . . . . . . . . . . . . . 115

7.2 Development of the proposed algorithm . . . . . . . . . . 117

7.2.1 Formulation of differential algebraic equations $\ldots \ldots 118$

7.2.2 Construction of the reduced-order model . . . . . . . . 120

7.2.3 Passivity preservation . . . . . . . . . . . . . . 124

7.3 Implementation issues $\ldots \ldots \ldots \ldots \ldots$

7.3.1 Boundary conditions . . . . . . . . . . . 128

7.3.2 Expansion point and error criteria . . . . . . . . . . 131

7.3.3 Complexity analysis $\ldots \ldots \ldots 131$

7.4 Numerical examples . . . . . . . . . . . . . . . . . 132

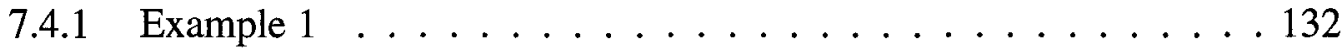

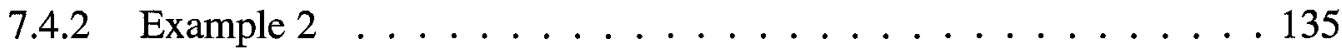

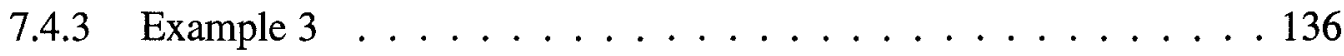

8 Passive Model-Order Reduction for RLC Circuits with Delay Elements 143

8.1 Introduction . . . . . . . . . . . . . . . . . . . 143

8.2 Networks with delay elements . . . . . . . . . . . . . . 145

8.3 Development of the proposed algorithm . . . . . . . . . . . 146

8.3.1 Formulation of ADDEs . . . . . . . . . . . . . 147

8.3.2 Construction of the reduced-order systems . . . . . . . . . . 148

8.3.3 Passivity of the reduced-order system . . . . . . . . . 149

8.4 Arnoldi-based construction of the orthonormal basis . . . . . . . . . . . 153

8.5 Numerical examples . . . . . . . . . . . . . . . 157

8.5.1 Validation of the implicit moment technique . . . . . . . . 157 
8.5.2 One-port network . . . . . . . . . . . . 158

8.5 .3 Two-port network . . . . . . . . . . . . . 161

9 Conclusions and Future Work $\quad 165$

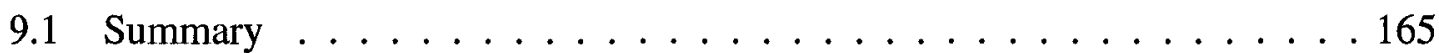

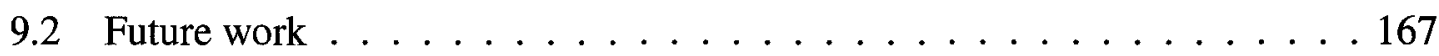

Appendix

A Proof of Lemma 3.2.5 169

B Proof of Proposition 3.3.1 172

$\begin{array}{ll}\text { C Proof of Propositions 4.3.2 and 4.3.3 } & 176\end{array}$

D Numerical Allocation of Passivity Violation 180

E Alternative Implementation Methodology for ICT 183

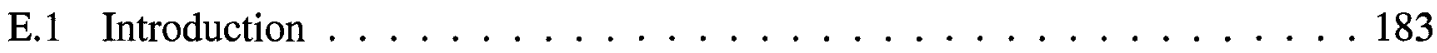

E.2 Proposed formulation . . . . . . . . . . . . . . 184

E.3 Proof of passivity preservation $\ldots \ldots \ldots \ldots 7$

E.4 Complexity analysis . . . . . . . . . . . . . . . . . 190

E.5 Numerical examples . . . . . . . . . . . . . . . . . 190

F Proof of Proposition 8.3.4 194

$\begin{array}{lll}\text { G Proof of Lemma 8.3.9 } & 196\end{array}$

$\begin{array}{ll}\text { References } & 197\end{array}$

$\mathrm{xi}$ 


\section{List of Tables}

3.1 Results of the last few N-R iterations before convergence to four zeroes of $\lambda_{1}(\jmath \omega)$. The 4 zero points are denoted by $\omega_{1}, \omega_{2}, \omega_{3}$ and $\omega_{4}$ and are described in each section of the table (Example 1). . . . . . . . . 46

3.2 CPU time comparison (seconds). . . . . . . . . . . . . 50

4.1 Numerical results for Example $3 \ldots \ldots \ldots$. . . . . . . . . . . . 70

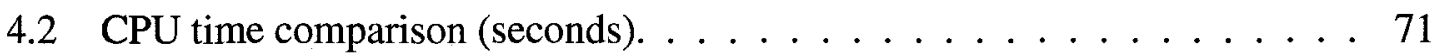

6.1 Transient simulation CPU time (seconds). . . . . . . . . . . . . 114

7.1 Frequency-dependent resistance and inductance matrices. The unit for the PUL parameters are $k \Omega / m$ and $\mu H / m$, respectively. $\left(R_{11}=R_{22}, L_{11}=\right.$ $\left.L_{22}, R_{12}=R_{21}, L_{12}=L_{21}\right) \ldots \ldots \ldots \ldots \ldots \ldots$

8.1 CPU speedup (seconds / seconds) $\ldots \ldots \ldots \ldots \ldots$ 


\section{List of Figures}

2.1 Series lumped model for a two-conductor transmission line. . . . . . . . . . 14

2.2 Series lumped model for a multi-conductor transmission line. . . . . . . . . 14

2.3 Sample MoC model implementation in $\mathrm{HSPICE}^{\circledR} \ldots \ldots \ldots$

2.4 Model realization for the product terms in $(2.18) \ldots \ldots \ldots$

3.1 Loss of passivity problem in MoC models. . . . . . . . . . . . . 31

3.2 Comparison of admittance matrices between the MoC model and the original TL network $($ Example 1$) . \ldots \ldots 43$

$3.3 \lambda_{1}(\jmath \omega)($ Example 1). . . . . . . . . . . . . . 45

3.4 Comparison of approximation and original data for $Y_{c}(s)$ and $P(s)$ (Exam-

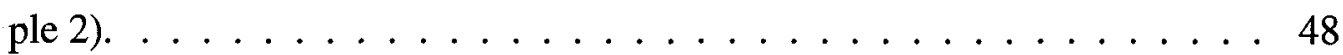

3.5 Comparison of admittance matrices between the MoC model and the original TL network (Example 2). . . . . . . . . . . . . . . . 49

3.6 Zoom of $\lambda_{1}(\jmath \omega)$ at small $\omega$ (Example 2). . . . . . . . . . . . . . . 49

3.7 Numerical results for Example $3 \ldots \ldots \ldots$. . . . . . . . . . . 51

4.1 Eigenvalues of the first submodel. . . . . . . . . . . . . 68

4.2 Eigenvalues of the second submodel. . . . . . . . . . . . 68

5.1 Illustration of the maximum passivity violation in a PVR. . . . . . . . . 74

5.2 Pseudocode of the proposed passivity enforcement. . . . . . . . . 80 
5.3 Eigenvalue spectrum of $\Re\{\tilde{\boldsymbol{Y}}(s)\}$ (Example 1). . . . . . . . . 82

5.4 Comparison of frequency responses, $\left|Y_{11}\right|($ Example 1) $\ldots \ldots 3$

5.5 Comparison of frequency responses, $\left|Y_{12}\right|($ Example 1$) \ldots \ldots 3$

5.6 Eigenvalue spectrum of $\Re\{\tilde{\boldsymbol{Y}}(s)\}$ (Example 2$) \ldots \ldots \ldots$

5.7 Comparison of frequency responses, $\left|\boldsymbol{Y}_{11}\right|$ (Example 2$) \ldots \ldots 5$

5.8 Comparison of frequency responses, $\left|\boldsymbol{Y}_{12}\right|$ (Example 2) $\ldots \ldots 6$

5.9 Comparison of frequency responses, $\left|\boldsymbol{Y}_{22}\right|$ (Example 2) $\ldots \ldots 6$

5.10 Eigenvalue spectrum of $\Re\{\tilde{\boldsymbol{Y}}(s)\}, \lambda_{1}$ (Example 3). . . . . . . 88

5.11 Eigenvalue spectrum of $\Re\{\tilde{\boldsymbol{Y}}(s)\}, \lambda_{2}$ (Example 3 ) . . . . . . . 89

5.12 Comparison of frequency responses, $\left|\boldsymbol{Y}_{11}\right|$ (Example 3). . . . . . . . 89

5.13 Comparison of frequency responses, $\left|Y_{13}\right|$ (Example 3). . . . . . . . . 90

5.14 Comparison of frequency responses, $\left|\boldsymbol{Y}_{16}\right|$ (Example 3). . . . . . . . . 90

6.1 An example of frequency-dependent variation of the PUL parameters. . . . 92

6.2 Impedance topology in approximating $\mathcal{Z}_{i j} \ldots \ldots \ldots 9$

6.3 Admittance topology in approximating $\mathcal{Y}_{i j} \ldots \ldots \ldots$. . . . . . . . 98

6.4 Lumped network realization for $\tilde{\mathcal{Z}}(s)$ of a four-conductor TL . . . . . . . 99

6.5 Lumped network realization for $\tilde{\mathcal{Y}}(s)$ of a four-conductor TL. . . . . . . 99

6.6 Network synthesis for $\tilde{\mathcal{Z}}_{11}(s)$ and $\tilde{\mathcal{Z}}_{22}(s)$ of the example impedance. . . . 101

6.7 Network synthesis for $\tilde{\mathcal{Z}}_{12}(s)$ and $\tilde{\mathcal{Z}}_{21}(s)$ of the example impedance. . . . . 101

6.8 Network synthesis for $\tilde{\mathcal{Z}}(s)$ based on Laplace elements. . . . . . . . . . 103

6.9 Network synthesis for $\tilde{\mathcal{Y}}(s)$ based on Laplace elements. . . . . . . . . . . 104

6.10 Circuit topology for Example $1 . \ldots \ldots$. . . . . . . . . . . . . . . . . .

6.11 Approximation of the PUL impedance and admittance. . . . . . . . . . 107

6.12 Transient responses for Example 1. . . . . . . . . . . . . 108

6.13 Approximation of the PUL impedance and admittance. . . . . . . . . . 109 
6.14 Circuit topology for Example $2 \ldots \ldots \ldots$. . . . . . . . 110

6.15 Transient responses for Example 2 . . . . . . . . . . . . . . . 111

6.16 Circuit topology for Example $3 . \ldots \ldots \ldots$. . . . . . . . . . . . . .

6.17 Transient responses at conductor 1 for the 6 -conductor network. . . . . . 112

6.18 Transient responses at conductor 4 for the 6 -conductor network. . . . . . 113

6.19 Transient responses at conductor 15 for the 25 -conductor network. . . . . . 114

7.1 Pseudocode for computing the orthonormal basis $\mathcal{Q}(x) \ldots \ldots \ldots$. . . . 122

7.2 A sample of the PUL impedance behavior along with the behavior of the equivalent network. . . . . . . . . . . . . . . . 134

7.3 Comparison of the ICT model and the TL stamp (Example 1). . . . . . . 135

7.4 Error comparison between the PRIMA and ICT models (Example 1). . . . 136

7.5 Circuit topology-1 for Example 2. . . . . . . . . . 136

7.6 Circuit topology-2 for Example 2. . . . . . . . . . . . 137

7.7 Time domain responses for the circuit network in Figure 7.5 . . . . . . 137

7.8 Time domain responses for the circuit network in Figure 7.6. . . . . . . . 138

7.9 Comparison of the ICT model and the TL stamp (Example 3). . . . . . . . 139

7.10 Error comparison between the PRIMA and ICT models (Example 3). . . . . 140

7.11 Circuit topology for Example $3 . \ldots \ldots 141$

7.12 Time domain responses for the PCB interconnect network. . . . . . . . . 142

8.1 A p-port RLC network with a lossless TL. . . . . . . . . . . . . . 145

8.2 Pseudocode for computing the orthonormal basis $\mathcal{Q} \ldots \ldots$. . . . . 157

8.3 Frequency-domain responses (Example 1) . . . . . . . . . . . . 159

8.4 Numerical performance of the reduced-order system (Example 2) . . . . 160

8.5 A two-port network (Example 3). . . . . . . . . . . . 161 
8.6 Frequency-domain responses (Example 3) . . . . . . . . . . . . . 162

8.7 Transient simulation results (Example 3). . . . . . . . . . . 163

E.1 Accuracy comparison of the proposed method, $\left|\boldsymbol{Y}_{11}\right| \ldots \ldots$. . . . . . 192

E.2 Accuracy comparison of the proposed method, $\left|\boldsymbol{Y}_{12}\right| \ldots \ldots$. . . . . 192

E.3 Eigenvalue plot of the real part of admittance matrix. . . . . . . . . 193 


\section{List of Abbreviations}

ADDEs

AFM

AWE

BCs

BP

CAD

$\mathrm{CFH}$

C-R

DAEs

DEPACT

F/B

FD-GEP

FD-PUL

GC

$\mathrm{GHz}$

GL

GPEP

HSPICE ${ }^{\circledR}$

ICT
Algebraic Delay-Differential Equations $\ldots \ldots \ldots \ldots \ldots \ldots \ldots$

Artifical Frequency Mapping $\ldots \ldots \ldots \ldots \ldots \ldots \ldots \ldots \ldots \ldots \ldots$

Asymptotic Waveform Evaluation $\ldots \ldots \ldots \ldots \ldots \ldots \ldots \ldots \ldots \ldots$

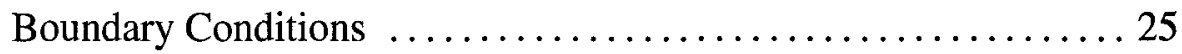

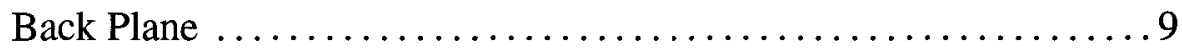

Computer-Aided Design $\ldots \ldots \ldots \ldots \ldots \ldots \ldots \ldots \ldots \ldots \ldots \ldots$

Complex Frequency Hopping $\ldots \ldots \ldots \ldots \ldots \ldots \ldots \ldots \ldots \ldots \ldots \ldots \ldots$

Cauchy-Riemann equations $\ldots \ldots \ldots \ldots \ldots \ldots \ldots \ldots \ldots \ldots$

Differential Algebraic Equations $\ldots \ldots \ldots \ldots \ldots \ldots \ldots \ldots \ldots$

Delay Extraction-Based Passive Compact Transmission-Line Macro-

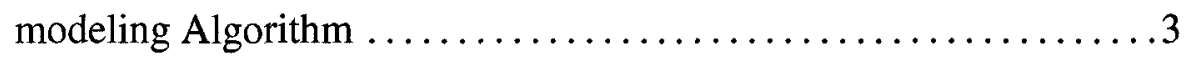

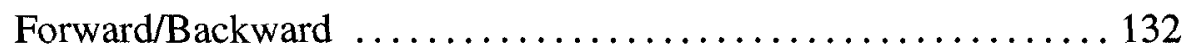

Frequency Dependent-Generalized Eigenvalue Problem ........ 33

Frequency-Dependent Per-Unit-Length $\ldots \ldots \ldots \ldots \ldots \ldots \ldots \ldots 4$

Conductor-Capacitor $\ldots \ldots \ldots \ldots \ldots \ldots \ldots \ldots \ldots \ldots \ldots \ldots . \ldots 9$

Giga-Hertz $\ldots \ldots \ldots \ldots \ldots \ldots \ldots \ldots \ldots \ldots \ldots \ldots \ldots \ldots$

Conductor-Inductor $\ldots \ldots \ldots \ldots \ldots \ldots \ldots \ldots \ldots \ldots \ldots$

Generalized Polynomial Eigenvalue Problem $\ldots \ldots \ldots \ldots \ldots \ldots 40$

A Circuit Simulator from Synopsys Inc. $\ldots \ldots \ldots \ldots \ldots \ldots \ldots$

Integrated Congruence Transform $\ldots \ldots \ldots \ldots \ldots \ldots \ldots \ldots \ldots$

xvii 
MATLAB $^{\circledR} \quad$ A Mathematical Analysis Package from MathWorks Inc.

$\mathrm{MoC}$

Method of Characteristics .3

MOR

Model-Order Reduction .3

MRA

Matrix Rational Approximation .3

MTL

PR

Positive-Real

PRIMA Passive Reduced-Order Interconnect Macromodeling Algorithm ...24

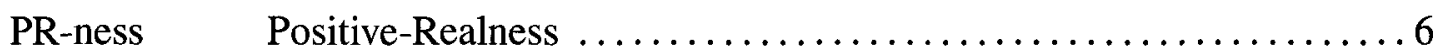

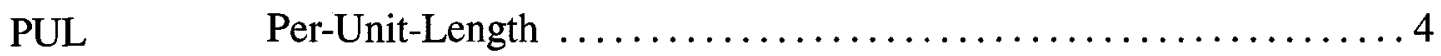

PVRs $\quad$ Passivity Violation Regions $\ldots \ldots \ldots \ldots \ldots \ldots \ldots \ldots \ldots \ldots \ldots \ldots$

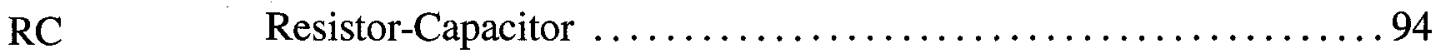

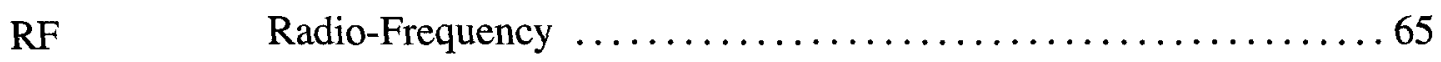

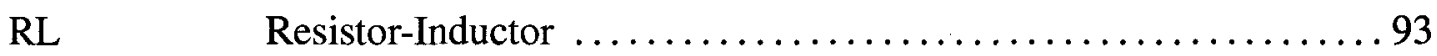

RLC $\quad$ Resistor-Inductor-Capacitor $\ldots \ldots \ldots \ldots \ldots \ldots \ldots \ldots \ldots \ldots \ldots \ldots$

xviii 
RLCM Resistor-Inductor-Capacitor-Mutual Inductor $\ldots \ldots \ldots \ldots \ldots \ldots 134$

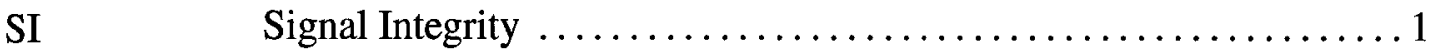

Spectre $^{\mathbb{R}} \quad$ A Circuit Simulator from Cadence Design Systems ........... 102

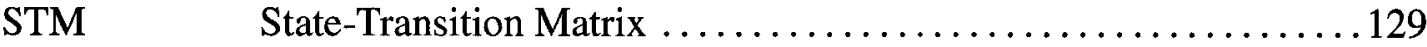

TEs Telegrapher's Equations $\ldots \ldots \ldots \ldots \ldots \ldots \ldots \ldots \ldots \ldots \ldots \ldots$

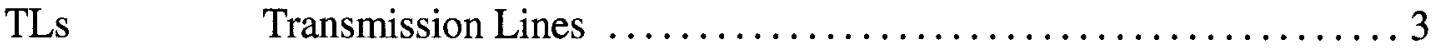

xix 


\section{List of Symbols}

\begin{tabular}{|c|c|}
\hline $\mathbb{R}, \mathbb{C}$ & Real, complex variable space \\
\hline $\boldsymbol{Y}(s)$ & Theoretical admittance matrix of a network \\
\hline$\tilde{\boldsymbol{Y}}(s)$ & Admittance of the approximant macromodel for a network \\
\hline$R, L, G, C$ & $\begin{array}{l}\text { Frequency-independent PUL resistance, inductance, conduc- } \\
\text { tance and capacitance of a transmission line }\end{array}$ \\
\hline $\boldsymbol{R}(s), \boldsymbol{L}(s), \boldsymbol{G}(s)$, & Frequency-dependent PUL resistance, inductance, conductance \\
\hline$C(s)$ & and capacitance of a transmission line \\
\hline $\mathcal{Z}(s) / \mathcal{Y}(s)$ & Theoretical PUL impedance/admittance of a transmission line \\
\hline$\tilde{\mathcal{Z}}(s) / \tilde{\mathcal{Y}}(s)$ & Approximant PUL impedance/admittance of a transmission line \\
\hline $\boldsymbol{Y}_{c}(s) / \tilde{\boldsymbol{Y}}_{c}(s)$ & Theoretical/approximant characteristic admittance \\
\hline $\mathcal{Z}_{o}$ & Characteristic impedance \\
\hline $\boldsymbol{P}(s) / \tilde{\boldsymbol{P}}(s)$ & Theoretical/approximant “delay-free” propagation function \\
\hline$d$ & Length of a transmission line \\
\hline$m$ & $\begin{array}{l}\text { Number of signal conductors in a }(m+1) \text {-conductor transmis- } \\
\text { sion line }\end{array}$ \\
\hline$I_{m}$ & An identity matrix with a size of $m \times m$ \\
\hline $\mathcal{Q}$ & Real orthonormal basis used in model-order reduction \\
\hline $\mathcal{Q}(x)$ & Orthonormal basis in the Hilbert space used in ICT-based MOR \\
\hline $\mathcal{L}(0, d)$ & Hilbert subspace \\
\hline$\left[\begin{array}{l}\boldsymbol{I}_{1}(s) \\
\boldsymbol{I}_{2}(s)\end{array}\right],\left[\begin{array}{l}\boldsymbol{V}_{1}(s) \\
\boldsymbol{V}_{2}(s)\end{array}\right]$ & $\begin{array}{l}\text { Terminal currents and voltages of a transmission line in the } s \text { - } \\
\text { domain }\end{array}$ \\
\hline
\end{tabular}




$$
\begin{array}{ll}
{\left[\begin{array}{l}
\boldsymbol{i}_{1}(t) \\
\boldsymbol{i}_{2}(t)
\end{array}\right],\left[\begin{array}{l}
\boldsymbol{v}_{1}(t) \\
\boldsymbol{v}_{2}(t)
\end{array}\right] \begin{array}{l}
\text { Terminal currents and voltages of a transmission line in the } \\
\text { time-domain }
\end{array}} \\
\begin{array}{ll}
\boldsymbol{i}_{p}(t), \boldsymbol{v}_{p}(t) & \text { Port currents and voltages of a network in the time-domain } \\
\boldsymbol{I}_{p}(s), \boldsymbol{V}_{p}(s) & \text { Port currents and voltages of a network in the } s \text {-domain } \\
\boldsymbol{C}_{\phi}, \boldsymbol{G}_{\phi}, \boldsymbol{b}_{\phi} & \text { MNA matrices for a lumped network } \phi \\
T_{h}(x) & h^{t h} \text { order Chebyshev polynomial of the first kind }
\end{array}
\end{array}
$$




\section{Chapter 1}

\section{Introduction}

\subsection{Background and motivation}

With the ever increasing operating frequency and circuit density, the high-speed interconnect becomes electrically long and behaves as a transmission line. As a result, the previously negligible interconnect effects, such as ringing, delay and crosstalk, become significant [1]. Accurate estimation of these effects has placed new demands on computer-aided design (CAD) tools to provide reliable assessment of signal integrity (SI) of the whole system. The fact that the constitutive equations used to represent the relation between the terminal voltages and currents of the interconnect are best described in the frequency domain, while the verification of SI in the presence of nonlinear circuit elements is a process that is typically done in the time-domain, results in a mixed frequency/time problem for the CAD tools. The widely adopted approach in solving this problem is aimed at constructing a time-domain realizable macromodel for the interconnect.

A typical macromodel for the interconnect should satisfy the following three requirements. Firstly, the macromodel should be accurate. This means that the transfer function of the macromodel should match the theoretical value of the original system within certain frequency range of interest. This is important to ensure the accurate circuit response when 
the macromodel is applied to describe the original system in the simulation. Secondly, the macromodel should be passive. The reason that passivity is an important factor to be preserved in a macromodel is that the interconnect is a passive component by its physical nature, whereas a non-passive macromodel, when connected to other passive terminals, may produce an unstable network, which may incur artificial oscillation during time-domain simulation. Thirdly, the macromodel should be of low order in order to minimize the computational cost when included in the circuit simulation during the SI verification process. Numerous techniques have been proposed in the literature to create macromodels for interconnects. Each technique displays some advantages and disadvantages depending on the operating frequency and the physical properties of interconnects. As a common property, most of them could produce an accurate macromodel within certain error tolerance with certain tradeoff, such as increasing the order of the macromodel. For a better understanding, some typical modeling and simulation techniques for the high-speed interconnects are briefly reviewed in the following two paragraphs. More details on each technique will be provided in Chapter 2.

The traditional modeling approach for the interconnect is based on the conventional series lumped model, where the interconnect is segmented into many sections and each section is represented by some lumped elements $[2,3]$, from which the time-domain description of the interconnect can be obtained. This approach provides a brute force solution to the mixed frequency- and time-domain problem. The passivity of the resulting macromodel based on this approach is ensured. However, this method usually leads to a large circuit, especially when the line is long. The order of the system matrices used to describe such a large circuit is high, which renders the simulation inefficient [4].

To overcome the difficulty of large order macromodels, a widely adopted approach in modeling interconnects relies on the fact that interconnects behave as delay operators for 
transmitted signals. This approach are derived from the well-known method of characteristics $(\mathrm{MoC})$, which was introduced by Branin in [5] to simulate lossless transmission lines (TLs). MoC techniques are based on extracting the minimum line propagation delay and produce exact models when applied to lossless lines [2]. These techniques have evolved over the years to handle coupled lossy lines [6-9]. The MoC-based approach derives its efficiency mainly from the capability to handle the inherent delay phenomena in the interconnects separately from the attenuation effects. This fact makes $\mathrm{MoC}$ an ideal choice in tackling transient simulation of long low-loss lines, since, in this case, delay represents the dominant interconnect effect, which can be easily captured using a simple delay source. The order of the resulting macromodel is usually small. However, one of the main disadvantages in using MoC-based macromodels in transient simulation of lossy interconnects is the potential loss of passivity in the developed macromodel. This problem presents a serious challenge in circuit simulators as mentioned above.

Delay extraction based passive compact transmission-line macromodeling algorithm (DEPACT) is another delay extraction-based macromodeling technique [10]. Based on a modified Lie formula [11], DEPACT segments the interconnect into many sections. Each section is composed of matrix rational approximation (MRA)-based sub-models and MoCbased sub-models. It has been demonstrated that DEPACT model is guaranteed to be passive and very efficient for long low-lossy transmission lines.

The excessive computational effort needed to perform the time-domain simulation on a large lumped model have prompted research efforts to focus on extracting a reduced-order macromodel for the large lumped circuit representing the interconnect structures. This process is called model-order reduction (MOR). An important group of MOR techniques for extracting the reduced-order model is based on moment-matching [12-17]. In that approach, the system transfer functions before and after reduction share the first few leading 
moments when expanded around certain points in the complex plane. Another notable technique of MOR is based on the concept of balancing truncation [18, 19]. The system is forced to a smaller size by removing some "weak" modes, whose contribution to the overall system is insignificant. One common requirement for the above techniques, when applied to a high-speed interconnect circuit, is that the interconnect must first be approximated by a finite, i.e., lumped, system with a large order, where those techniques can be used to obtain a reduced-order system.

A recent macromodeling approach of interconnects is aimed at developing a compact, or reduced-order, macromodel without having to represent the interconnect with a large lumped circuit. The motivation behind this approach is that the large lumped circuit is only an approximation to the interconnect over a limited frequency band and represents only an intermediate step to reach the final macromodel. In order to circumvent this step, the concept of integrated congruence transform (ICT) was introduced in [20]. ICT works by first constructing an orthonormal basis for the Hilbert subspace of the voltages/currents moments across the interconnects, and then uses this orthonormal basis as the transformation operator to obtain the macromodel directly from the Telegrapher's equations (TEs). The ICT technique was further developed in [21], where it was shown that finding the orthonormal basis without explicitly computing the moments leads to a more numerically robust technique in constructing the macromodel. It was also shown in [22] that these techniques can be extended to perform sensitivity analysis. Nonetheless, the ICT technique in its current form can only handle interconnects with frequency-independent Per-Unit-Length (PUL) parameters. This situation excludes an important segment of interconnect circuitry that is characterized by frequency-dependent PUL (FD-PUL) parameters, which are necessary to account for geometric and ohmic loss-induced interconnect dispersion [23, 24]. As an example, at high frequencies, the edge effects cause the currents in the interconnect 
conductors to be concentrated toward the sharp edge. This, in turn, increases the resistance. In other words, this edge effect contributes to the dependence of the PUL resistance on frequency. It has been shown in the literature, such as [2,25-27], that neglecting these frequency-dependency effects leads to significant errors in simulation results.

Another important problem in SI analysis emerges to handle large lumped networks along with delay elements when modeling interconnects using different techniques, such as lumped models, ICT and DEPACT. In such a situation, the entire circuit network may consist of large equivalent sub-circuits with lumped components, sub-systems described in state space from ICT models, and delay-based sub-circuits represented by lossless lines from DEPACT models etc. Although the performance of the signal integrity in this situation can be improved if the advantages of each macromodeling technique are explored to model appropriate portions of interconnect individually, a direct analysis on the entire network may still be computationally expensive. As discussed above, the concept of MOR has been well developed to address the efficiency issue in large lumped circuit simulation. This idea was also further developed in [28] to handle the situation where there exist subsystems described in state space in the lumped networks. However, the presence of delay elements presents difficulties that make MOR a challenging task on various levels. It is plausible to argue that applying MOR to circuits with delay elements can be approached through partitioning the large circuits into multi-port subnetworks around the delay sources and then applying multi-port MOR on each subnetwork. This approach, however, would then have to face the difficulty of dealing with many ports, which is a challenging task as reported in [29]. 


\subsection{Objectives and contributions}

In order to address those issues stated in Section 1.1, this thesis is aimed at developing a compact macromodel for high-speed networks in the presence of different types of interconnects. The resulting macromodel is guaranteed passive and can be embedded in a non-linear circuit simulator for SI analysis. The specific contributions of the thesis are as follows,

1. A novel theory is developed that addresses the issue of passivity in the delay-based macromodels of electrical interconnects constructed based on method of characteristics. The philosophy behind the novel theory is based on deriving the necessary and sufficient conditions for the loss of positive-realness (P-R-ness) in the admittance matrix of the MoC-based macromodel. An algorithmic procedure is proposed to check for passivity violation regions in the macromodels. The result presented can be employed to test for PR-ness in dynamical systems described by algebraic delaydifferential equations (ADDEs). The work from this contribution resulted in two IEEE Transactions papers and one conference paper [30-32].

2. An efficient passivity enforcement algorithm is derived to correct the passivity violations of a non-passive MoC macromodel. The derived algorithm is based on the firstorder perturbation performed on the eigenvalues of the real part of the admittance matrix. The negative eigenvalues are perturbed to be greater or equal to zero while minimizing the $L_{2}$-norm of the deviation. The proposed algorithm can handle the MoC model for a transmission line with both two conductors and multi-conductors. One IEEE Transactions paper [33] resulted from this work has been submitted for publication and one conference was published [34].

3. An efficient circuit modeling approach is developed for the frequency-dependent 
PUL parameters. The developed approach works in a two-step process. In the first step, the frequency-dependent PUL parameters (characterized at discrete frequency points) are approximated using positive-real (PR) rational functions. The approximation minimizes the relative error at each discrete frequency point. In the second step, a new synthesis methodology based on Laplace elements is proposed to realize the resulting rational functions into networks that can be adopted by many interconnect macromodeling techniques, such as series lumped model, MRA, DEPACT, and waveform relaxation. The results from this contribution have been published in three IEEE Transactions papers [35-37]. Another paper is also in preparation.

4. A new algorithm based on ICT is proposed to construct a compact reduced-order macromodel for the high-speed interconnects with frequency-dependent parameters. The proposed algorithm provides a new approach to perform implicit moment matching on a system described by a mixed set of linear differential algebraic equations (DAEs). This feature offers a high accuracy in the reduced-order macromodel over a wide frequency range. Two methodologies are presented to implement the proposed algorithm. The passivity of the reduced-order models from both implementations is guaranteed. This contribution resulted in two IEEE Transactions papers [35, 37] and two conference papers $[38,39]$.

5. A new algorithm is proposed for model-order reduction of networks with delayelements. The proposed algorithm shows that the delay elements can be formulated in a way that leads to passivity preserving reduced-order models. In addition, a significant size reduction can be achieved through using an Arnoldi-like algorithm that avoids the numerical inefficiency resulting from using the MGS process directly. This contribution results in one IEEE Transactions paper [40] and two conference papers $[41,42]$. 


\subsection{Organization}

This thesis is organized as follows. Chapter 2 presents a brief review on some macromodeling and model-order reduction techniques for the interconnect networks. The concept of passivity for electrical systems is also discussed in this chapter. Chapter 3 presents the new theory that checks the passivity of MoC-based macromodels for the case of two-conductor transmission lines. Chapter 4 describes a generalized theory that checks the passivity of MoC-based macromodel for the case of multi-conductor TLs. Chapter 5 presents the robust passivity enforcement algorithm that performs the passivity violations correction for non-passive MoC-based macromodels. Chapter 6 describes the modeling approach developed to approximate the frequency-dependent PUL parameters by rational functions, and to realize the rational functions into circuit networks. Chapter 7 describes the new macromodeling algorithm for frequency-dependent interconnects based on ICT. Chapter 8 describes the new MOR algorithm for networks with delay elements. Chapter 9 presents the summary of the thesis and discusses the future work. 


\section{Chapter 2}

\section{Review of Macromodeling and MOR Techniques}

\subsection{Introduction}

Interconnects are common electrical signal propagation paths, that exist at various levels of an electrical system, such as on-chip, packaging structures, printed circuit boards (PCBs) and back planes (BPs) $[4,43]$. At low system operating frequencies, interconnects behave as short circuits. When the frequency increases up to Giga-Hertz $(\mathrm{GHz})$ range as in modern high-speed systems, these interconnects become electrically long, and hence, behave as transmission lines (for this reason, the words "interconnect" and "transmission line" are used interchangeably in this thesis). In order to perform the signal integrity analysis of the whole electrical system, the previously negligible interconnect effects coming from these transmission lines have to be taken into account. A transmission line is best described in the frequency-domain using Telegrapher's equations. This presents a serious problem in the SI analysis with the presence of non-linear terminations, which can only be described in the time-domain [1].

As discussed in Chapter 1, many macromodeling techniques have been proposed in the 
literature to address this mixed frequency- and time-domain problem in circuit simulation in the presence of high-speed interconnects and non-linear terminations. Each technique offers different advantages and disadvantages depending on the structure of the TL and the operating frequency on the TL. To facilitate the presentation, the frequency-domain description of a general TL is first presented in the following paragraph.

A multi-conductor transmission line (MTL) with $m+1$ conductors (including $m$ signal conductors and one ground conductor) is described in the Laplace-domain using Telegrapher's equations [2],

$$
\boldsymbol{T} \frac{\partial}{\partial x} \boldsymbol{X}(x, s)=-[\mathcal{N}(s)+s \mathcal{M}(s)] \boldsymbol{X}(x, s)
$$

where $\boldsymbol{T}=\left[\begin{array}{cc}\mathbf{0} & \boldsymbol{I}_{m} \\ \boldsymbol{I}_{m} & \mathbf{0}\end{array}\right], \mathcal{N}(s)=\left[\begin{array}{cc}\boldsymbol{R}(s) & \mathbf{0} \\ \mathbf{0} & \boldsymbol{G}(s)\end{array}\right], \mathcal{M}(s)=\left[\begin{array}{cc}\boldsymbol{L}(s) & \mathbf{0} \\ \mathbf{0} & \boldsymbol{C}(s)\end{array}\right], \boldsymbol{X}(x, s)=$ $\left[\begin{array}{l}\boldsymbol{I}(x, s) \\ \boldsymbol{V}(x, s)\end{array}\right] \cdot \boldsymbol{V}(x, s)$ and $\boldsymbol{I}(x, s) \in \mathbb{C}^{m \times 1}$ are, respectively, the Laplace-domain voltages and currents of the transmission line at an arbitrary point $x$ along the line, and $\boldsymbol{R}(s), \boldsymbol{L}(s)$, $\boldsymbol{G}(s)$ and $\boldsymbol{C}(s) \in \mathbb{C}^{m \times m}$ denote the PUL resistance, inductance, conductance and capacitance matrices. Laplace variable $s$ is explicitly specified in $\boldsymbol{R}(s), \boldsymbol{G}(s), \boldsymbol{L}(s)$ and $\boldsymbol{C}(s)$ to emphasize that the PUL parameters may be frequency-dependent for a general case. $\boldsymbol{I}_{m}$ is an identity matrix with a dimension of $m \times m$. The main goal of various macromodeling techniques is aimed at seeking an approximated relation relating the terminal voltages and currents of the transmission line in terms of a time-domain realizable model, as demonstrated in (2.2).

$$
\left[\begin{array}{l}
\boldsymbol{I}_{1}(s) \\
\boldsymbol{I}_{2}(s)
\end{array}\right] \approx \tilde{\boldsymbol{Y}}(s)\left[\begin{array}{l}
\boldsymbol{V}_{1}(s) \\
\boldsymbol{V}_{2}(s)
\end{array}\right]
$$


where $\left[\begin{array}{c}\boldsymbol{I}_{1}(s) \\ \boldsymbol{I}_{2}(s)\end{array}\right]=\left[\begin{array}{c}\boldsymbol{I}(0, s) \\ -\boldsymbol{I}(d, s)\end{array}\right]$ and $\left[\begin{array}{c}\boldsymbol{V}_{1}(s) \\ \boldsymbol{V}_{2}(s)\end{array}\right]=\left[\begin{array}{c}\boldsymbol{V}(0, s) \\ \boldsymbol{V}(d, s)\end{array}\right]$ denote the currents and voltages, respectively, at the near end $(x=0)$ and the far end $(x=d)$ of the TL, with $d$ being the length of the TL. A minus sign is put in front of $\boldsymbol{I}(d, s)$ just to make the current flowing into the TL positive. $\tilde{\boldsymbol{Y}}(s)$ is the approximated macromodel that can be analytically transferred into time-domain. $\tilde{\boldsymbol{Y}}(s)$ in the context of (2.2) denotes the admittance matrix of the macromodel. For the convenience of presentation, $\boldsymbol{Y}(s)$ will be used in this thesis to denote the exact admittance matrix of the transmission line.

This chapter presents a brief review on some macromodeling and MOR techniques, upon which the contributions of this thesis are built. Section 2.2 first discusses the concept of passivity, which is an important factor in evaluating a macromodeling or MOR technique. Section 2.3 describes the topology of the series lumped model for those TLs with frequency-independent parameters, followed by an introduction of MRA. Section 2.4 presents the method of characteristic as well as DEPACT technique, which use MoC models for lossless TLs. Section 2.5 reviews the typical passivity enforcement techniques in compensating the passivity violations for a non-passive system described by a statespace representation. Section 2.6 reviews the methodology of moment-matching MOR techniques in constructing the reduced-order models for resistor-inductor-capacitor (RLC) type circuits. Section 2.7 presents the ICT-based macromodeling technique for frequencyindependent TLs.

\subsection{Passivity conditions}

Passivity is an important property of many electrical systems. A passive system implies that the system cannot generate more energy than it absorbs. By construction, transmission line is a passive component. Hence, its macromodel must be passive. Passivity is a stronger 
condition than stability. In other words, a stable model may not be passive. However, a passive model must be stable. When a stable but non-passive model is connected to other passive terminations, the overall system may become unstable $[14,44]$. The loss of passivity in the macromodel of a passive component, therefore, can be a serious problem for the overall system simulation. A specific example demonstrating this problem can be found in [44].

A system is passive if and only if its immittance ${ }^{1}$ matrix is positive-real $[20,45,46]$. In general, an admittance matrix $\boldsymbol{Y}(s)$ is said to be positive-real if it satisfies the following conditions $[20,45-48]$ :

1. $\boldsymbol{Y}(s)$ is analytic for all values of $s$ with $\Re(s)>0$;

2. $\boldsymbol{Y}\left(s^{\star}\right)=\boldsymbol{Y}^{\star}(s)$ where $(\star)$ is the complex conjugate operator;

3. $\boldsymbol{Y}^{T}\left(s^{\star}\right)+\boldsymbol{Y}(s)$ is a non-negative definite matrix, that is,

$$
\boldsymbol{z}^{\star T}\left[\boldsymbol{Y}^{T}\left(s^{\star}\right)+\boldsymbol{Y}(s)\right] \boldsymbol{z} \geq 0 \quad \forall s \in\{s \mid \Re\{s\}>0\} \text { and } \forall \boldsymbol{z}
$$

The first condition implies that $Y(s)$ does not have poles in the right half plane. The third condition implies that the real part of $\boldsymbol{Y}(s)$ must be non-negative definite for all $s$ in the right half plane. Direct verification on those three conditions for an admittance matrix is usually difficult. Some simplified conditions are available in the literature for some special cases. For example, when $\boldsymbol{Y}(s)$ is a rational matrix function, the first condition is automatically satisfied if the third condition is true. The second condition is true if all the coefficients of the rational function are real [46]. Another simplified version of positiverealness conditions can be found in Chapter 3, when presenting a new theory in checking the passivity of MoC-based macromodels.

\footnotetext{
${ }^{1}$ The term "immittance matrix" refers to either impedance or admittance matrix.
} 
For the convenience of presentation, the rest of this subsection provides the definition of analyticity for a complex function, as well as some lemmas which are useful for the proofs of several theorems in this thesis $[49,50]$.

Definition 2.2.1 A complex (possibly matrix-valued) function $\mathcal{H}(s)$ is analytic on a region $\mathfrak{R}$ if its real and imaginary parts, respectively, denoted by $\mathcal{H}_{\Re}(\sigma, \omega)$ and $\mathcal{H}_{\Im}(\sigma, \omega)$, have continuous first partial derivatives at every point in $\mathfrak{R}$ and satisfy the Cauchy-Riemann equations $(C-R)$,

$$
\frac{\partial \mathcal{H}_{\Re}(\sigma, \omega)}{\partial \sigma}=\frac{\partial \mathcal{H}_{\Im}(\sigma, \omega)}{\partial \omega} \text { and } \frac{\partial \mathcal{H}_{\Re}(\sigma, \omega)}{\partial \omega}=-\frac{\partial \mathcal{H}_{\Im}(\sigma, \omega)}{\partial \sigma}
$$

where s here is assumed to be given by $s=\sigma+\jmath \omega$.

Lemma 2.2.1 If $\mathcal{H}(s)$ is a constant matrix, then both $\mathcal{H}(s)$ and $s \mathcal{H}(s)$ are analytic $\forall s \in$ $\mathbb{C}$.

Lemma 2.2.2 If $\Lambda$ is diagonal and constant matrix, then $e^{-s \Lambda}$ is analytic $\forall s \in \mathbb{C}$.

Lemma 2.2.3 If $\mathcal{G}(s)$ and $\mathcal{H}(s)$ are two analytic functions on a given region $\mathfrak{R}$, then so are $\mathcal{G}(s)+\mathcal{H}(s)$ and $\mathcal{G}(s) \mathcal{H}(s)$.

Lemma 2.2.4 If $\mathcal{G}(s)$ is analytic matrix-valued function and $\mathcal{G}^{-1}(s)$ is non-singular within a given region of the s-domain, then $\mathcal{G}^{-1}(s)$ is also analytic within the same region.

The proofs of the above lemmas can be deduced in a straightforward manner and therefore are omitted for brevity.

\subsection{Lumped element based models}

The transmission line is considered as a distributed circuit component since it is electrically long. The series lumped model is proposed based on the assumption that if we can segment 
the TL into many sections with each section being electrically-short (ideally to be a zero length), then each section can be characterized with lumped components. To simplify the presentation, Figure 2.1 shows a series lumped model for a two-conductor TL [2]. The relation in (2.2) can then be easily obtained from writing the modified nodal analysis (MNA) equations for the lumped model [51]. The extension of series lumped models to multiconductor TLs is straightforward [2]. For brevity, a simplified version depicted in blocks is demonstrated in Figure 2.2, where block 1 and block 2 denote the PUL impedance and admittance networks, respectively. The series lumped model provides a brute force ap-

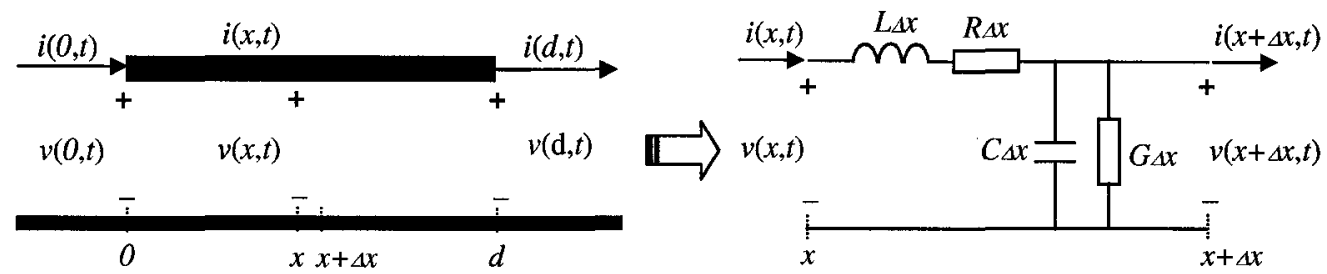

Figure 2.1: Series lumped model for a two-conductor transmission line.

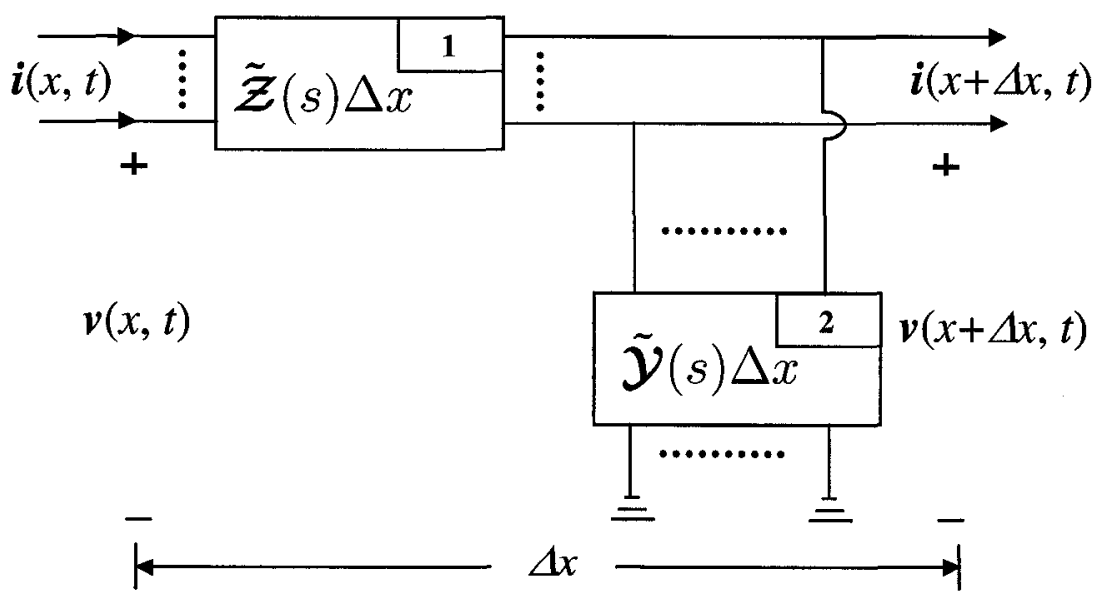

Figure 2.2: Series lumped model for a multi-conductor transmission line.

proximation to transmission lines. The accuracy of the model is determined by the number of sections. A more accurate model can be obtained with a greater number of sections. This will lead to large system matrices, rendering the circuit simulation a prohibitive task. On 
the other hand, the passivity of the system can be preserved through this modeling process, since all components of the model are passive elements. As a fundamental approach, the series lumped model provides an extensive reference for a comparison with other advanced macromodeling techniques [3,52-57], and it has been incorporated into SPICE-like CAD tools, for instance, the "U" element in HSPICE ${ }^{\circledR}$.

Compared to the series lumped model, matrix rational approximation (MRA) builds the macromodel in an optimal way [4]. From the TEs in (2.1), the exponential stamp of the TL, which relates the terminal voltages and currents, can be stated to be

$$
\left[\begin{array}{c}
\boldsymbol{V}(d, s) \\
-\boldsymbol{I}(d, s)
\end{array}\right]=e^{\mathbb{Z}}\left[\begin{array}{c}
\boldsymbol{V}(0, s) \\
\boldsymbol{I}(0, s)
\end{array}\right]
$$

where,

$$
\mathbb{Z}=-(\mathbb{A}(s)+\mathbb{B}(s)) d, \quad \mathbb{A}(s)=\left[\begin{array}{cc}
\mathbf{0} & \boldsymbol{R}(s) \\
\boldsymbol{G}(s) & \mathbf{0}
\end{array}\right], \quad \mathbb{B}(s)=\left[\begin{array}{cc}
\mathbf{0} & \boldsymbol{L}(s) \\
\boldsymbol{C}(s) & \mathbf{0}
\end{array}\right]
$$

MRA approximates the exponential stamp in (2.4) using a rational function in the following form:

$$
e^{\mathbb{Z}}=\mathbb{P}(\mathbb{Z})^{-1} \mathbb{Q}(\mathbb{Z})
$$

where $\mathbb{P}(\mathbb{Z})$ and $\mathbb{Q}(\mathbb{Z})$ are polynomial matrices. The coefficients of the polynomials are constant and pre-determined. Once the rational function is obtained, the formulation of the macromodels can be derived analytically. Upon some conditions, MRA models are guaranteed to be passive by construction. However, in the presence of large delay lines (e.g. long line with small losses), the order of the rational approximation may be high, leading to inefficient transient simulation. 


\subsection{Delay extraction based models}

The method of characteristic based macromodel $[9,30]$ for the TL is constructed based on the solution of the TEs (2.1) for the terminal voltages and currents, which can be reduced to the following equations:

$$
\begin{aligned}
& \boldsymbol{I}_{1}(s)=\boldsymbol{Y}_{c}(s) \boldsymbol{V}_{1}(s)-\boldsymbol{J}_{1}(s) \\
& \boldsymbol{I}_{2}(s)=\boldsymbol{Y}_{c}(s) \boldsymbol{V}_{2}(s)-\boldsymbol{J}_{2}(s)
\end{aligned}
$$

where $\boldsymbol{J}_{1}(s)$ and $\boldsymbol{J}_{2}(s)$ are given by,

$$
\begin{aligned}
& \boldsymbol{J}_{1}(s)=\boldsymbol{Q}(s)\left[\boldsymbol{Y}_{c}(s) \boldsymbol{V}_{2}(s)+\boldsymbol{I}_{2}(s)\right] \\
& \boldsymbol{J}_{2}(s)=\boldsymbol{Q}(s)\left[\boldsymbol{Y}_{c}(s) \boldsymbol{V}_{\mathbf{1}}(s)+\boldsymbol{I}_{1}(s)\right]
\end{aligned}
$$

$\boldsymbol{Y}_{c}(s)$ is the characteristic admittance matrix defined by,

$$
\boldsymbol{Y}_{c}(s)=\boldsymbol{\Gamma}^{-1}(s)(\boldsymbol{G}(s)+s \boldsymbol{C}(s))
$$

and $\boldsymbol{\Gamma}(s)$ is a "delay-factor" matrix given by, $\boldsymbol{\Gamma}(s)=\sqrt{(\boldsymbol{G}(s)+s \boldsymbol{C}(s))(\boldsymbol{R}(s)+s \boldsymbol{L}(s))}$ with $\boldsymbol{Q}(s)$ being obtained from $\boldsymbol{Q}(s)=e^{-\boldsymbol{\Gamma}(s) d}$.

An efficient approach to constructing the time-domain macromodel is based on finding a suitable rational approximation for $\boldsymbol{Y}_{c}(s)$ and another one for the "delay-free" matrix $\boldsymbol{P}(s)$ which is obtained from $\boldsymbol{Q}(s)$ using

$$
\boldsymbol{P}(s)=e^{s \tau} \boldsymbol{M}^{-1} \boldsymbol{Q}(s) \boldsymbol{M}
$$

In (2.12), $M$ and $\tau$ are the eigenvector and eigenvalue matrices of the matrix $d \sqrt{C_{\infty} L_{\infty}}$ where $C_{\infty}$ and $L_{\infty}$ are, in respective order, the PUL capacitance and inductance matrices 
at $s \rightarrow \infty$. Assume $\tilde{\boldsymbol{Y}}_{c}(s)$ and $\tilde{\boldsymbol{P}}(s)$ refer to the approximations of both $\boldsymbol{Y}_{c}(s)$ and $\boldsymbol{P}(s)$, respectively. Combining (2.7)-(2.12), it can be demonstrated that the admittance matrix of the resulting macromodel is given by

$$
\tilde{\boldsymbol{Y}}(s)=\left[\begin{array}{ll}
\boldsymbol{W}_{0}^{-1}(s) \boldsymbol{W}_{1}(s) \tilde{\boldsymbol{Y}}_{c}(s) & \boldsymbol{W}_{0}^{-1}(s) \boldsymbol{W}_{2}(s) \tilde{\boldsymbol{Y}}_{c}(s) \\
\boldsymbol{W}_{0}^{-1}(s) \boldsymbol{W}_{2}(s) \tilde{\boldsymbol{Y}}_{c}(s) & \boldsymbol{W}_{0}^{-1}(s) \boldsymbol{W}_{1}(s) \tilde{\boldsymbol{Y}}_{c}(s)
\end{array}\right]
$$

where,

$$
\begin{aligned}
& \boldsymbol{W}_{0}(s)=\boldsymbol{I}_{m}-\boldsymbol{M} e^{-s \boldsymbol{\tau}} \tilde{\boldsymbol{P}}(s) e^{-s \tau} \tilde{\boldsymbol{P}}(s) \boldsymbol{M}^{-1} \\
& \boldsymbol{W}_{1}(s)=\boldsymbol{I}_{m}+\boldsymbol{M} e^{-s \boldsymbol{\tau}} \tilde{\boldsymbol{P}}(s) e^{-s \tau} \tilde{\boldsymbol{P}}(s) \boldsymbol{M}^{-1} \\
& \boldsymbol{W}_{2}(s)=-2 \boldsymbol{M} e^{-s \boldsymbol{\tau}} \tilde{\boldsymbol{P}}(s) \boldsymbol{M}^{-1}
\end{aligned}
$$

For the case of two-conductor TLs, (2.13) can be simplified to be a $2 \times 2$ matrix, which is also denoted here by $\tilde{\boldsymbol{Y}}(s)$ and can be written as

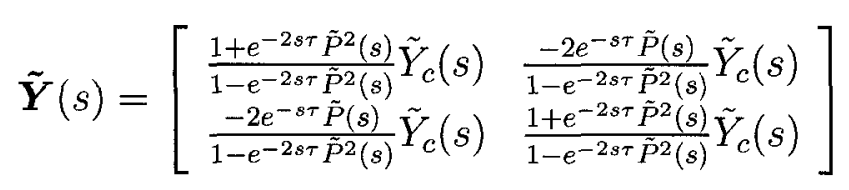

where $\tau$ is the scalar form of $\tau$, and $\tilde{P}(s)$ and $\tilde{Y}_{c}(s)$ are scalar rational approximation of $P(s)$ and $Y_{c}(s)$, respectively. Figure 2.3 shows a sample implementation scheme of the MoC model using Laplace elements in HSPICE ${ }^{\circledR}$ for a two-conductor TL.

MoC-based approaches feature a powerful advantage which results from extracting the line minimum delay from the propagation function without compromising the causality of the resulting macromodel. This feature provides an efficient approach to simulate long low-loss lines. However, passivity of the resulting macromodel for lossy TLs is not always guaranteed.

On the other hand, DEPACT $[10,58]$ combines the merits of both MoC and MRA, leading to passive macromodels. It employs a mechanism for delay extraction prior to 


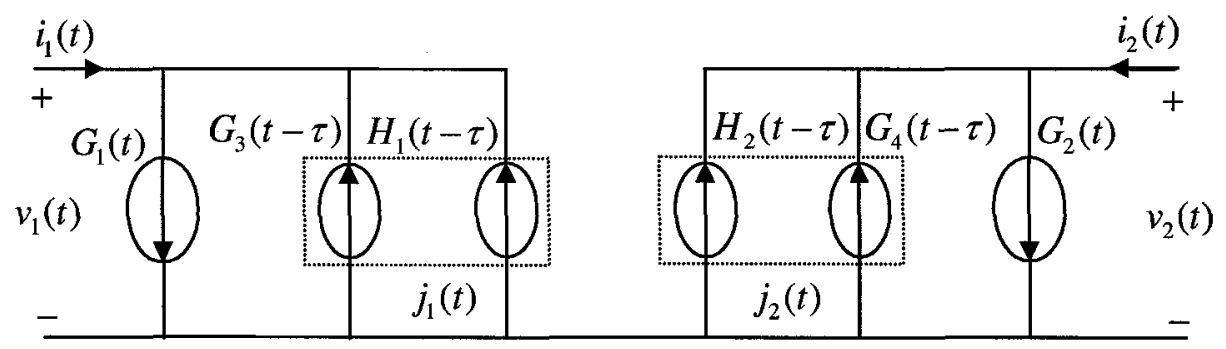

$G_{1}(t)$ and $G_{2}(t)$ represent the voltage control current sources coming from $\tilde{Y}_{c}(s)$ $G_{3}(t)$ and $G_{4}(t)$ represent the voltage control current sources coming from $\tilde{P}(s) \tilde{Y}_{c}(s)$ $H_{1}(t)$ and $\mathrm{H}_{2}(t)$ represent the current control current sources coming from $\widetilde{P}(s)$

Figure 2.3: Sample MoC model implementation in HSPICE ${ }^{\circledR}$.

performing matrix rational approximation. Specifically, instead of approximating the exponential term in (2.4) by a rational function directly, DEPACT proposes a modified Lie formula in the following form:

$$
e^{-[\mathbb{A}(s)+s \mathbb{B}(s)] d} \approx \prod_{k=1}^{M}\left[e^{-\frac{\mathbb{B}_{\infty}}{2 M} d} e^{-\frac{\mathrm{A}(s)+\mathbb{B}(s)-\mathbb{B}_{\infty}}{M} d} e^{-\frac{\mathbb{B}_{\infty}}{2 M} d}\right],
$$

where $\mathbb{B}_{\infty}=\left[\begin{array}{cc}\mathbf{0} & \boldsymbol{L}_{\infty} \\ \boldsymbol{C}_{\infty} & \mathbf{0}\end{array}\right] . M$ is a user defined value that determines the number of sections in DEPACT model. It is proven that the error in the approximation of (2.18) in given by

$$
\|\epsilon\| \approx O\left(1 / M^{2}\right)
$$

Figure 2.4 shows the macromodel realization of the product terms in (2.18). The lossless parts are synthesized with MoC models, which are exact in this case. MRA model is utilized to model the middle part in Figure 2.4. The DEPACT model for the full TL consists of $M$ cascaded sections. Each section is represented by Figure 2.4. It has been reported that for relatively short and lossy lines, delay extraction may reduce the efficiency of the raw MRA macromodels. In this case, series lumped model or MRA may be better to model 
the TL.

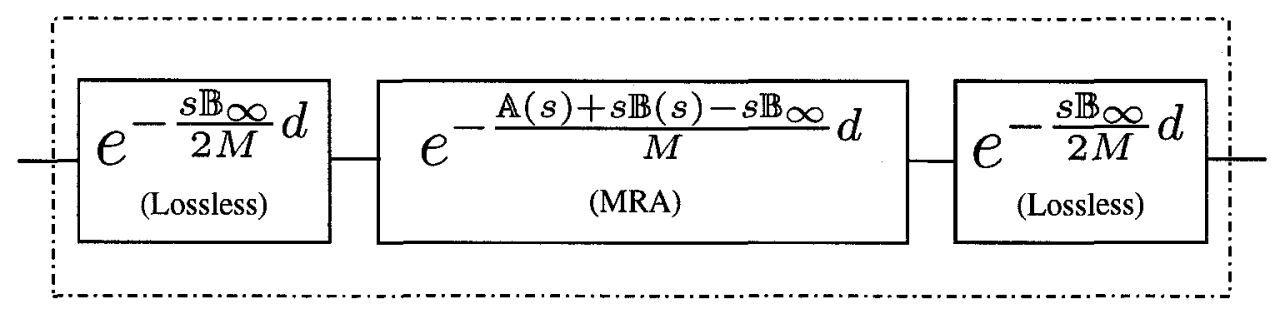

Figure 2.4: Model realization for the product terms in (2.18).

\subsection{Passivity enforcement}

The importance of passivity in the macromodels for interconnect networks has been extensively discussed in the literature. However, non-passive macromodels still exist in various cases. One typical case happens when a network is characterized by tabulated data obtained either from measurement or rigorous full-wave electromagnetic simulations. To include the tabulated data into non-linear circuit for transient simulation, one usually approximates the tabulated data by rational functions and subsequently synthesizes a SPICE-compatible macromodel from such an approximation [59]. Ensuring the passivity in the macromodel is one of the key issues for the approximation. Although several passivity guaranteed rational approximation algorithms, such as the ones based on convex optimization, exist, they can be CPU extensive and may not be practically feasible $[59,60]$. Other approximation algorithms, such as [61-63], are widely used due to its computational efficiency. However, the resulting macromodels based on these approaches are usually stable, but not passive. This motivates the development of new passivity enforcement algorithm used to compensate the passivity violations such that the resulting macromodel becomes passive.

In order to introduce the passivity enforcement algorithm, we write the approximated 
stable rational function, or the macromodel, into state-space as described in (2.20).

$$
\left\{\begin{array}{l}
\dot{\boldsymbol{x}}_{e}(t)=\boldsymbol{A}_{e} \boldsymbol{x}_{e}(t)+\boldsymbol{B}_{e} \boldsymbol{u}_{e}(t) \\
\boldsymbol{y}_{e}(t)=\boldsymbol{C}_{e} \boldsymbol{x}_{e}(t)+\boldsymbol{D}_{e} \boldsymbol{u}_{e}(t)
\end{array}\right.
$$

where $\boldsymbol{A}_{e} \in \mathbb{R}^{N_{e} \times N_{e}}, \boldsymbol{B}_{e} \in \mathbb{R}^{N_{e} \times n_{e}}, \boldsymbol{C}_{e} \in \mathbb{R}^{n_{e} \times N_{e}}, \boldsymbol{D}_{e} \in \mathbb{R}^{n_{e} \times n_{e}} . n_{e}$ and $N_{e}$ are, in a respective order, the numbers of ports in the system and its associated state variables. It is noted that the state-space representation in (2.20) can also be referred to a general system described by ordinary differential equations (ODEs). Denote $\boldsymbol{H}_{e}(s)$ to be the transfer function of the system, then

$$
\boldsymbol{H}_{e}(s)=\boldsymbol{C}_{e}\left(s-\boldsymbol{A}_{e}\right)^{-1} \boldsymbol{B}_{e}+\boldsymbol{D}_{e}
$$

The goal of passivity enforcement is to perturb certain parameters in (2.21) such that $\boldsymbol{H}_{e}(s)$ becomes positive-real. $\boldsymbol{C}_{e}$ matrix is usually chosen to be perturbed for this purpose due to the reasons stated in [64]. A typical passivity enforcement algorithm is an iterative process. Each iteration consists of two main steps. In the first step, the passivity of the macromodel is verified and the passivity violation regions, if they exist, are identified. This step can be succeeded by checking the eigenvalues of the Hamiltonian matrix, $M_{e}$, associated with the system (2.20).

$$
\boldsymbol{M}_{e}=\left[\begin{array}{cc}
\boldsymbol{A}_{e}-\boldsymbol{B}_{e}\left(\boldsymbol{D}_{e}+\boldsymbol{D}_{e}^{T}\right)^{-1} \boldsymbol{C}_{e} & \boldsymbol{B}_{e}\left(\boldsymbol{D}_{e}+\boldsymbol{D}_{e}^{T}\right)^{-1} \boldsymbol{B}_{e}^{T} \\
-\boldsymbol{C}_{e}^{T}\left(\boldsymbol{D}_{e}+\boldsymbol{D}_{e}^{T}\right)^{-1} \boldsymbol{C}_{e} & -\boldsymbol{A}_{e}^{T}+\boldsymbol{C}_{e}^{T}\left(\boldsymbol{D}_{e}+\boldsymbol{D}_{e}^{T}\right)^{-1} \boldsymbol{B}_{e}^{T}
\end{array}\right]
$$

If $M_{e}$ does not have the imaginary eigenvalues, then the system is passive. Otherwise, the imaginary eigenvalues delimit the boundaries of the passivity violation regions [59, $64,65]$. This will invoke the second step of the algorithm for passivity compensation. Grivet-Talocia and Huang etc. [64] address this step by perturbing $C_{e}$ matrix such that the imaginary eigenvalues of $M_{e}$ are perturbed and eliminated at the end. On the other hand, 
Saraswat and Achar etc. $[59,66]$ start from the definition of passivity in Section 2.2, and perturb the $\boldsymbol{C}_{e}$ matrix such that the eigenvalues of the real part of $\boldsymbol{H}_{e}(s)$ become nonnegative. Both methods adopt the first-order perturbation theory and hence they work well for systems with small passivity violations $[59,64,66]$. The $L_{2}$-norm of the deviation in the system response due to the perturbation is minimized in the process to optimize the perturbation.

\subsection{Model-order reduction}

The macromodel of the interconnect usually results in a large size of system matrices, which make the direct transient simulation inefficient. To address this issue, various MOR algorithms are introduced in the literature. The theory behind MOR is that the large size of system matrices incurs a large number of poles, out of which only few are dominant. In other words, there are many non-dominant poles, whose contribution to the system response is minor and can be neglected. By capturing only the dominant poles, the reduced model can significantly reduce the transient simulation time without compromising accuracy.

There are two main groups of MOR techniques. One is based on moment-matching [12-16, 20, 56, 67-78]. In that approach, the system transfer functions before and after reduction share the first few leading moments when expanded around certain points in the complex plane. The other one is based on balanced truncation $[18,19,79,80]$, which is often used in the second-level reduction process due to its high computational cost and will not be reviewed in this section. Asymptotic waveform evaluation (AWE) works by expanding the system transfer function into a finite order Taylor series, computing the first leading coefficients (also called moments) of the series, then transferring the series into a rational function. This approach suffers from serious numerical problems. As a result, 
the maximum order of useful moment is usually too small to capture the system response. To address this issue, multi-point AWE techniques, such as complex frequency hopping (CFH) [72], are proposed. However, an important disadvantage of these techniques is that the resulted model may not be passive. Krylov subspace-based techniques have been shown to be able to preserve the passivity in addition to the first leading moments in the reducedorder model [14]. Hence, the rest of this section will focus on this kind of techniques. To this end, the general lumped network formulation is first presented in the following paragraphs $[28,81]$.

Consider a $p$-port linear network $\phi$ consisting of lumped RLC components, which can be expressed using the MNA equations

$$
\begin{aligned}
\boldsymbol{C}_{\phi} \dot{\boldsymbol{x}}_{\phi}(t)+\boldsymbol{G}_{\phi} \boldsymbol{x}_{\phi}(t) & =\boldsymbol{b}_{\phi} \boldsymbol{v}_{p}(t) \\
\boldsymbol{i}_{p}(t) & =\boldsymbol{b}_{\phi}^{T} \boldsymbol{x}_{\phi}(t)
\end{aligned}
$$

where $\boldsymbol{x}_{\phi}(t) \in \mathbb{R}^{N_{\phi}}$ is the vector of node voltage waveforms appended by waveforms of independent voltage source currents and inductor currents; $\mathbb{R}^{N_{\phi}}$ is the node-space of the network $\phi ; \boldsymbol{b}_{\phi} \in \mathbb{R}^{N_{\phi} \times p}$ is a selector matrix, which maps the port voltages into the node space of the network; $\boldsymbol{v}_{p}(t)$ and $\boldsymbol{i}_{p}(t) \in \mathbb{R}^{p}$ define the port voltages and currents of the network, respectively; $\boldsymbol{C}_{\phi}$ and $\boldsymbol{G}_{\phi} \in \mathbb{R}^{N_{\phi} \times N_{\phi}}$ are constant matrices describing lumped memory and memoryless elements of the network, respectively [28,82]. More specifically, $C_{\phi}$ and $G_{\phi}$ are structured in the following form

$$
\boldsymbol{C}_{\phi}=\left[\begin{array}{cc}
\boldsymbol{C}_{a} & 0 \\
0 & C_{b}
\end{array}\right], \quad \boldsymbol{G}_{\phi}=\left[\begin{array}{cc}
\boldsymbol{N}_{\phi} & \boldsymbol{E}_{\phi} \\
-\boldsymbol{E}_{\phi}^{T} & 0
\end{array}\right]
$$

where $C_{a}, C_{b}$ and $N_{\phi}$ are nonnegative definite matrices containing stamps of capacitors, inductors and resistors, respectively. $\boldsymbol{E}_{\phi}$ corresponds to current variables in a Kirchoff's current law (KCL) formulation.

The reduced-order system using Krylov subspace technique is obtained by replacing the 
state variables $\boldsymbol{x}_{\phi}$ with $\mathcal{Q} \hat{\boldsymbol{x}}_{\phi}$, where $\hat{\boldsymbol{x}}_{\phi}$ is a vector containing the new state variables in the reduced-order system, and $\mathcal{Q}$ is an orthonormal basis in $\mathbb{R}^{N_{\phi} \times n} \quad\left(n \ll N_{\phi}\right)$, which spans the first $\left\lfloor\frac{n}{p}\right\rfloor$ block moments of $\boldsymbol{X}_{\phi}(s)$, where $\boldsymbol{X}_{\phi}(s)$ is the Laplace-domain representation for $x_{\phi}(t)$. The reduced-order system can then be written as

$$
\begin{aligned}
\hat{\boldsymbol{C}}_{\phi} \dot{\hat{\boldsymbol{x}}}_{\phi}(t)+\hat{\boldsymbol{G}}_{\phi} \hat{\boldsymbol{x}}_{\phi}(t) & =\hat{\boldsymbol{b}}_{\phi} \boldsymbol{v}_{p}(t) \\
\boldsymbol{i}_{p}(t) & =\hat{\boldsymbol{b}}_{\phi}^{T} \hat{\boldsymbol{x}}_{\phi}(t)
\end{aligned}
$$

where $\hat{\boldsymbol{C}}_{\phi}=\mathcal{Q}^{T} \boldsymbol{C}_{\phi} \mathcal{Q} \in \mathbb{R}^{n \times n}, \hat{\boldsymbol{G}}_{\phi}=\mathcal{Q}^{T} \boldsymbol{G}_{\phi} \mathcal{Q} \in \mathbb{R}^{n \times n}$ and $\hat{\boldsymbol{b}}_{\phi}=\mathcal{Q}^{T} \boldsymbol{b}_{\phi} \in \mathbb{R}^{n \times p}$.

Computation of the orthonormal basis $\mathcal{Q}$ represents one of the main computational issues of Krylov subspace-based MOR algorithms. One way to compute $\mathcal{Q}$ is through the explicit computation of the moment, $M_{i}$, of $\boldsymbol{X}_{\phi}(s)$ using the following recursive relationships

$$
M_{0}=-G_{\phi}^{-1} b_{\phi} ; \quad M_{i}=G_{\phi}^{-1} C_{\phi} M_{i-1}
$$

and then adopting the modified Gram-Schmidt (MGS) process to obtain the orthonormal basis for their spanning subspace. This approach, however, leads to numerical difficulties making the inclusion of higher-order moments of no impact in enhancing the accuracy of reduced-order model [21]. Another approach, which is referred to in this thesis as the implicit approach, uses an adaptation of the MGS process known as the Arnoldi algorithm that avoids the explicit computation of the moments. It should be noted that the key point that enables adapting the MGS to the Arnoldi algorithm is the recursive relation between the moments indicated by (2.26). Another issue that is worth pointing out is that structuring the $\boldsymbol{G}_{\phi}$ and $\boldsymbol{C}_{\phi}$ matrices as in (2.24) represents the key idea in preserving the passivity of the reduced-order model. The proof of passivity preservation as well as details of the Arnoldi algorithm to construct $\mathcal{Q}$ can be found in [14], where a passive reduced-order interconnect 
macromodeling algorithm, called PRIMA, is introduced.

Arnoldi algorithm can usually offer a good reduction. However, it requires that the TL must first be approximated by a finite, i.e. lumped, system with a large order. This approximation process can be avoided by using ICT technique, which is reviewed in the following section.

\subsection{Integrated congruence transform}

ICT is aimed at developing a compact, reduced-order macromodel without having to represent the interconnect with a large lumped circuit. The motivation behind this approach is that large lumped circuit is only an approximation to the interconnect over a limited frequency band and represents only an intermediate step to reach the final macromodel. ICT builds the macromodel from the TEs directly into the following form [21]:

$$
\begin{aligned}
(\hat{\mathcal{N}}+s \hat{\mathcal{M}}) \hat{\boldsymbol{X}}(s) & =\hat{\boldsymbol{b}} \tilde{\boldsymbol{V}}(s) \\
\tilde{\boldsymbol{I}}(s) & =\hat{\boldsymbol{b}}^{T} \hat{\boldsymbol{X}}(s)
\end{aligned}
$$

where $\tilde{\boldsymbol{V}}(s)$ and $\tilde{\boldsymbol{I}}(s)$ are vectors containing the approximate terminal voltages and currents, respectively, i.e.,

$$
\tilde{\boldsymbol{V}}(s) \approx\left[\begin{array}{c}
\boldsymbol{V}(0, s) \\
\boldsymbol{V}(d, s)
\end{array}\right], \quad \tilde{\boldsymbol{I}}(s) \approx\left[\begin{array}{c}
\boldsymbol{I}(0, s) \\
-\boldsymbol{I}(d, s)
\end{array}\right]
$$

$\hat{\boldsymbol{X}}(s)$ is an auxiliary set of variables that are added to complete the description of the system. The construction of the matrices in (2.27) can be described in a two-step process. In the first step, an orthonormal basis is constructed for the Hilbert subspace of the first few derivatives of $\mathcal{X}(x, s)$ w.r.t. $s$, where $\mathcal{X}(x, s)$ is defined in the next paragraph. This orthonormal basis is then used in the second step to obtain $\hat{\mathcal{N}}, \hat{\mathcal{M}}$ and $\hat{b}$ from the PUL matrices via projection in Hilbert space. 


\section{Step 1. Construction of the orthonormal basis}

We define $\mathcal{X}(x, s) \in \mathbb{C}^{2 m \times 2 m}$ as the solution matrix for the TEs that is obtained under a set of $2 m$ boundary conditions (BCs). Specifically, the $i^{t h}$ column of $\mathcal{X}(x, s)$ denotes the solution associated with the $i^{\text {th }} \mathrm{BC}$, which is specified by ensuring that the $2 m$ terminal voltages are given by the $i^{\text {th }}$ column in a $2 m \times 2 m$ identity matrix. Using a Taylor series expansion around point $s_{o}$ in the Laplace-domain, we can write $\mathcal{X}(x, s)$ in the following form:

$$
\mathcal{X}(x, s)=\sum_{i=0}^{\infty} \boldsymbol{U}^{(i)}(x)\left(s-s_{o}\right)^{i}
$$

where $U^{(i)}(x) \in \mathbb{C}^{2 m \times 2 m},(0 \leq z \leq d)$ are the $x$-dependent Taylor series coefficient matrices, which are typically referred to as moments or scaled derivatives. These moments can be thought of as elements in Hilbert space denoted by $\mathcal{L}(0, d)$. The orthonormal basis $\mathcal{Q}(x)$ is required to span the Hilbert subspace of the first, say $q$, of such moments, i.e., $\mathcal{Q}(x)$ spans $\left\{\boldsymbol{U}^{(0)}(x), \cdots, \boldsymbol{U}^{(q-1)}(x)\right\}$. Similar to Krylov subspace MOR, there are two main approaches for computing $\mathcal{Q}(x)$. They are referred to as explicit and implicit approaches. In the explicit approach, $U^{(i)}(x)$ 's are first computed explicitly before using an orthonormal process, such as MGS, to obtain the orthonormal basis. Computing $\boldsymbol{U}^{(0)}(x), \cdots, \boldsymbol{U}^{(q-1)}(x)$ is done by first substituting (2.29) into (2.1) and equating similar powers of $s$ to obtain,

$$
\begin{aligned}
& \mathcal{T}\left(s_{o}\right) \boldsymbol{U}^{(0)}(x)=\mathbf{0} \\
& \mathcal{T}\left(s_{o}\right) \boldsymbol{U}^{(i)}(x)=-\mathcal{M} \boldsymbol{U}^{(i-1)}(x) \quad i>0
\end{aligned}
$$

where $\mathcal{T}\left(s_{o}\right)=\left(\boldsymbol{T} \frac{d}{d x}+\mathcal{N}+s_{o} \mathcal{M}\right), \mathcal{M}=\left[\begin{array}{ll}\boldsymbol{L} & \mathbf{0} \\ \mathbf{0} & \boldsymbol{C}\end{array}\right]$ and $\mathcal{N}=\left[\begin{array}{cc}\boldsymbol{R} & \mathbf{0} \\ \mathbf{0} & \boldsymbol{G}\end{array}\right]$. It is noted that the PUL parameters of the TL are assumed to be frequency-independent here. (2.30) 
is solved once, whereas (2.31) is solved recursively $q-1$ times. This explicit approach is extended in [21] to the implicit approach by making use of the simple iterative relationship between $\boldsymbol{U}^{(i)}(x)$ and $\boldsymbol{U}^{(i-1)}(x)$. It has been shown that both the implicit and explicit approaches yield $\mathcal{Q}(x)$ matrix that theoretically spans the same subspace of $\left\{\boldsymbol{U}^{(0)}(x), \cdots\right.$, $\left.\boldsymbol{U}^{(q-1)}(x)\right\}$. However, implicit approach has much better numerical properties than explicit approach. The reasons behind this fact and numerical results that demonstrate it can be found in [21].

\section{Step 2. Computation of $\hat{\mathcal{N}}, \hat{\mathcal{M}}$ and $\hat{b}$}

Computation of matrices $\hat{\mathcal{N}}, \hat{\mathcal{M}}$ and $\hat{b}$ defining the reduced system in (2.27) is a step that is carried out in the ICT framework. It is typically performed by using orthonormal basis $\mathcal{Q}(x)$, computed as described above, to cast the Telegrapher's equations (2.1) into the form of (2.27). For brevity, we state here the basic definitions of $\hat{\mathcal{N}}, \hat{\mathcal{M}}$ and $\hat{b}$ in terms of $\mathcal{Q}(x), \mathcal{N}, \mathcal{M}$ and $T$ as follows: $\hat{\mathcal{M}}=\int_{0}^{d} \mathcal{Q}(x)^{T} \mathcal{M} \mathcal{Q}(x) d x, \hat{\mathcal{N}}=\hat{\mathcal{N}}_{1}+\hat{\mathcal{N}}_{2}$, $\hat{\mathcal{N}}_{1}=\int_{0}^{d} \mathcal{Q}(x)^{T} \mathcal{N} \mathcal{Q}(x) d x, \hat{\mathcal{N}}_{2}=\hat{\boldsymbol{T}}-\mathcal{U}, \mathcal{U}=\mathcal{Q}_{i}(d)^{T} \mathcal{Q}_{v}(d)-\mathcal{Q}_{i}(0)^{T} \mathcal{Q}_{v}(0), \hat{\boldsymbol{T}}=$ $\int_{0}^{d} \mathcal{Q}(x)^{T} \boldsymbol{T} \frac{d \mathcal{Q}(x)}{d x} d x, \hat{\boldsymbol{b}}=\left[\mathcal{Q}_{i}^{T}(0)-\mathcal{Q}_{i}^{T}(d)\right]^{T}$, and $\mathcal{Q}_{i}(x)$ and $\mathcal{Q}_{v}(x) \in \mathbb{R}^{m \times(2 m q)}$ denote the portions of $\mathcal{Q}(x)$ corresponding to the current and voltage variables, respectively.

The above discussion focused on the application of ICT to develop the compact model for the TLs with frequency-independent PUL matrices. However, the application of ICT to the more general case of FD-PUL matrices presents several challenging issues. 


\section{Chapter 3}

\section{Passivity Verification in Delay-Based Macromodels of Two-Conductor Transmission Lines}

\subsection{Introduction}

The method of characteristics provides an efficient approach for modeling interconnects in that the minimum signal delay on the TL can be represented using delay sources. However, an MoC-based macromodel is not guaranteed to be passive. Passivity is an important property that needs to be preserved in the macromodels. With a non-passive macromodel, the transient simulation may suffer artificial oscillation.

In this chapter, a new theory is proposed to tackle the issue of passivity in MoC-based macromodels [30]. The new theory is established through deriving the necessary and sufficient conditions for a given macromodel to be passive. The developed theory enables a simple algebraic test to determine whether the model is passive or that a loss of passivity has occurred. In addition, the presented theory features a useful computational advantage in that if there is a violation of passivity, then the proposed test can provide the exact locations in the Laplace domain at which those violations occur. It has been shown recently that 
accurate identification of passivity violation points is a key requirement for compensation of loss of passivity $[59,64,83]$. Although it might be argued that one possible technique to identify those locations can be done through brute-force calculations of eigenvalues of the real part of the admittance matrix while changing the value of the frequency variable $s$, this technique remains impractical since it requires sweeping the frequency to very large values (ideally infinity) while taking infinitesimally small steps.

It is also important to note that other approaches have been proposed to verify passivity and identify locations of passivity violations $[64,83]$. However, those approaches are restricted to systems described by delay-free ordinary differential equations. MoC-based macromodels, on the other hand, include delay elements and are represented in the timedomain by a set of algebraic differential equations. In that sense, a MoC macromodel is represented by a system of algebraic delay differential equations. This fact makes the presented theory a good candidate to tackle the issue of passivity of general dynamical systems described by ADDEs [84].

To keep the mathematical presentation of the underlying theory fairly simple, the discussion in this chapter will be focused on the case of two-conductor transmission line, where one of the conductors is serving as the ground return conductor. Generalization to multi-conductor will be presented in Chapter 4. The chapter is organized as follows. Section 3.2 presents a brief discussion on the passivity of MoC-based macromodels. The basic construction steps for MoC-models can be found in Chapter 2. Section 3.3 presents the theoretical basis in developing the proposed theory. The numerical implementation of the proposed algorithm is described in Section 3.4. The computational complexity of the implementation is discussed in Section 3.5. Section 3.6 presents numerical experiments to validate the accuracy of the proposed algorithm. 


\subsection{Passivity of MoC-based macromodels}

It can be noted from Section 2.2 that a network is passive if and only if its immittance matrix satisfies three conditions of PR-ness. It is usually impractical to have a passivity check based on those three conditions for the obvious reason that $\boldsymbol{Y}(s)$ has to be examined for all the points, $s$, in the right half of the complex plane. However, and without loss of generality, those three conditions can be simplified through some basic assumptions on the admittance matrix of MoC models, denoted by $\tilde{\boldsymbol{Y}}(s)$ in (2.17). This can be summarized in the following lemma.

Lemma 3.2.5 Assume that the MoC-based model is asymptotically stable, (i.e., $\tilde{\boldsymbol{Y}}(s)$ has no singular points for $\Re(s) \geq 0$ ) and that both of $\tilde{Y}_{c}(s)$ and $\tilde{P}(s)$ do not have poles in the right-half plane of the $s$-domain with $\tilde{P}(s)$ being a strict rational function ${ }^{1}$. Then $\tilde{\boldsymbol{Y}}(s)$ satisfies the first two conditions of being a PR matrix. Furthermore, the third condition is satisfied in $\Re(s)>0$ if it is satisfied on the imaginary axis $(\Re(s)=0)$. In other words, the third condition (2.3) for PR-ness can be replaced by

$$
\boldsymbol{z}^{\star T}\left[\tilde{\boldsymbol{Y}}^{T}(-\jmath \omega)+\tilde{\boldsymbol{Y}}(\jmath \omega)\right] \boldsymbol{z} \geq 0 \quad \forall \omega \in \mathbb{R} \text { and } \forall \boldsymbol{z} \in \mathbb{C}^{2 m}
$$

For the purpose of this chapter, we assume that an MoC-based model is stable and that both $\tilde{Y}_{c}(s)$ and $\tilde{P}(s)$ have no poles for $\Re(s) \geq 0$ with $\tilde{P}(s)$ being a strict rational function. The latter assumption is in no way a restricting assumption since it is always satisfied as a requirement in the initial phase of fitting both of $Y_{c}(s)$ and $P(s)$ to construct the model. The proof of Lemma 3.2.5 is present in Appendix A.

To illustrate the loss of passivity in MoC-based macromodels, a $5 \mathrm{~cm}$ lossy PCB transmission line with frequency-dependent parameters is considered [85]. Figure 3.1(a) shows

\footnotetext{
${ }^{1} \mathrm{~A}$ strict rational function $\psi(s)$ satisfies the condition that $\psi(s)_{s \rightarrow \infty}=0$
} 
frequency responses obtained from the $\mathrm{MoC}$ macromodel and that obtained from the transmission line circuit stamp. As can be seen, both responses match accurately up to $4.3 \mathrm{GHz}$. Passivity of the macromodel is verified by computing the eigenvalues of the real part of the admittance matrix as a function of frequency. Figure 3.1(b) shows the frequency spectrum of one of the eigenvalues and it clearly demonstrates the passivity violation, since the eigenvalue takes negative values at higher frequency.

\subsection{Theoretical basis}

It is clear from the discussion in the previous section that checking the passivity of $\tilde{\boldsymbol{Y}}(s)$ can be approached by verifying whether it satisfies the condition expressed in (3.1). This, however, still bears little practical value since it requires sweeping the frequency variable, $s$, to very high value (ideally $j \infty$ ) while taking infinitesimally small steps. The proposed approach aims at addressing this issue through developing a new algorithm to check for passivity of MoC macromodels. This section lays out the theoretical basis behind the proposed algorithm. Development of the underlying theory will proceed in three consecutive stages that can be summarized as follows.

1. In the first stage, the $\mathrm{MoC}$ macromodel is represented in the time-domain by a system of algebraic delay-differential equations (ADDEs). This part will be explained in Subsection 3.3.1.

2. In the second stage, the necessary and sufficient condition for an $\mathrm{MoC}$ model to be passive is presented via a novel theorem in Subsection 3.3.2.

3. The third stage, which is given in Subsection 3.3.3, is concerned with identifying the locations on the imaginary axis where a non-passive $\mathrm{MoC}$ model violates the passivity condition in (3.1). 


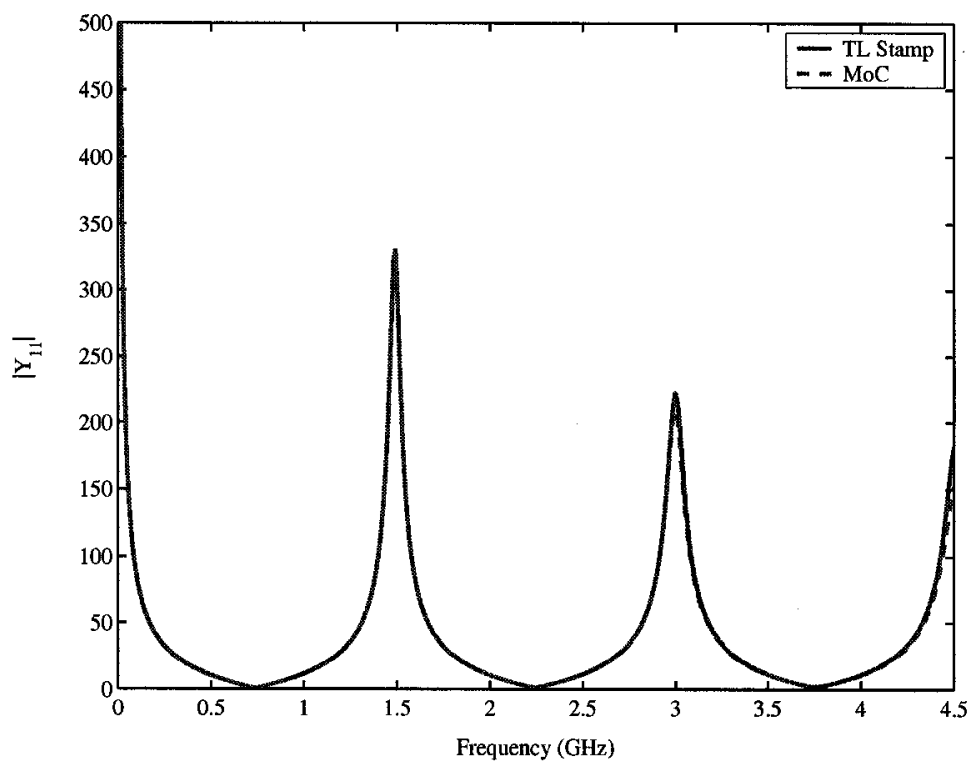

(a) Frequency-domain comparison between the MoC model and the distributed (accurate) model.

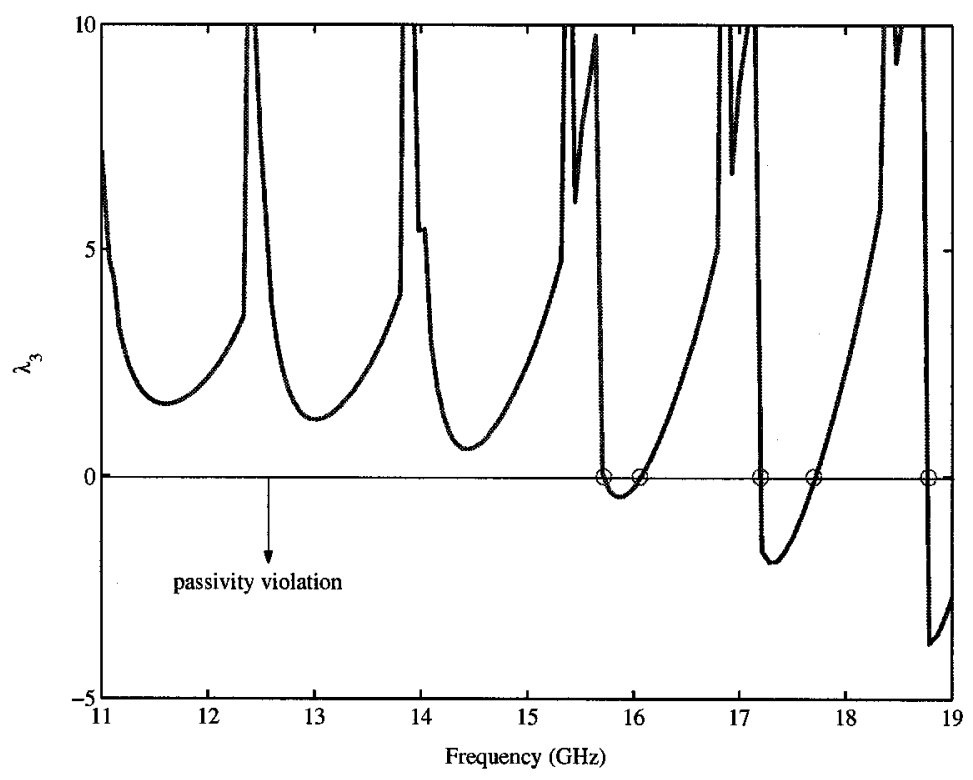

(b) $3^{\text {rd }}$ Eigenvalue of $\Re\{\tilde{\boldsymbol{Y}}(s)\}$.

Figure 3.1: Loss of passivity problem in MoC models. 


\subsubsection{Formulation of ADDE's}

To facilitate the passivity verification of $\tilde{\boldsymbol{Y}}(s)$, the proposed approach formulates a set of coupled algebraic delay differential equations, whose transfer function is identical to $\tilde{\boldsymbol{Y}}(s)$. A systematic way to obtain this system is given by the following proposition.

Proposition 3.3.1 An MoC model for 2-conductor TL represented by (2.17) can be described in the time-domain by a system of ADDEs, $\Sigma_{\tau}$, defined as follows,

$$
\Sigma_{\tau}:\left\{\begin{aligned}
\mathcal{K} \dot{\boldsymbol{\chi}}(t) & =\mathcal{A} \boldsymbol{\chi}(t)+\mathcal{P} \chi(t-2 \tau)+\mathcal{B} \tilde{\boldsymbol{v}}(t) \\
\tilde{\boldsymbol{i}}(t) & =\mathcal{C} \chi(t)+\mathcal{Q}_{1} \chi(t-\tau)+\mathcal{Q}_{2} \chi(t-2 \tau)+\mathcal{D} \tilde{\boldsymbol{v}}(t)
\end{aligned}\right.
$$

where

- matrices $\mathcal{K}, \mathcal{A}, \mathcal{P}, \mathcal{B}, \mathcal{C}, \mathcal{Q}_{1}, \mathcal{Q}_{2}$ and $\mathcal{D}$ are given in terms of the coefficients of the rational approximations, $\tilde{P}(s)$ and $\tilde{Y}_{c}(s)$. Detailed description of these matrices can be found in Appendix B,

- $\tilde{\boldsymbol{i}}(t) \approx\left[\begin{array}{l}i_{1}(t) \\ i_{2}(t)\end{array}\right]$ and $\tilde{\boldsymbol{v}}(t) \approx\left[\begin{array}{l}v_{1}(t) \\ v_{2}(t)\end{array}\right]$ are vectors containing the approximate timedomain terminal currents and voltages respectively, and

- $\chi(t)$ is a vector of state variables.

Furthermore, let $\Pi(s)$ be the Laplace-domain transfer matrix between $i(t)$ and $v(t)$, i.e.,

$$
\begin{array}{r}
\Pi(s)=\left(\mathcal{C}+\mathcal{Q}_{1} e^{-s \tau}+\mathcal{Q}_{2} e^{-2 s \tau}\right) \\
\left(s \mathcal{K}-\mathcal{A}-\mathcal{P} e^{-2 s \tau}\right)^{-1} \mathcal{B}+\mathcal{D}
\end{array}
$$

then,

$$
\Pi(s)=\tilde{\boldsymbol{Y}}(s) \quad \forall s \in \mathbb{C}
$$

The proof of the above proposition is given in Appendix B. 


\subsubsection{Passivity verification in MoC macromodels}

Proposition 3.3.1 indicates that the passivity of an MoC-based macromodel can be verified alternatively via checking the passivity of the delay system $\Sigma_{\tau}$. The issue of verifying the passivity of systems described by delay equations has been addressed recently in the literature $[86,87]$. These techniques use a series of linear matrix inequalities (LMIs)-based approaches to identify passivity violation in delay systems. However, these methods suffer the following disadvantages when applied to systems representing electrical interconnects, such as $\Sigma_{\tau}$.

- Although these techniques can detect the passivity violation, they are incapable of pinpointing where this violation occurs. It has been shown recently that accurate determination of passivity violation is a crucial step needed to enforce the passivity at these regions $[64,83]$.

- LMI techniques are mainly iterative techniques, with each iteration requiring a computational complexity of $O\left(N^{6}\right)$ [88], where $N$ is the size of the ADDE system. This cost can become computationally expensive, especially for systems with multiple conductors.

Theorem 3.3.1 presented below establishes a new approach for verifying the passivity of $\Sigma_{\tau}$.

Theorem 3.3.1 An MoC macromodel (or system $\Sigma_{\tau}$ ) is passive if and only if there does not exist any simple purely imaginary values for $s$ that satisfy the following frequency dependent generalized eigenvalue problem (FD-GEP),

$$
s \boldsymbol{K} \boldsymbol{\xi}(s)=\boldsymbol{H}(s) \boldsymbol{\xi}(s)
$$


where

$$
\boldsymbol{H}(s)=\mathcal{V}+\boldsymbol{W}_{11} e^{s \tau}+\boldsymbol{W}_{21} e^{2 s \tau}+\boldsymbol{W}_{12} e^{-s \tau}+\boldsymbol{W}_{22} e^{-2 s \tau}
$$

and

$$
\begin{aligned}
& \boldsymbol{K}=\left[\begin{array}{cc}
\mathcal{K} & 0 \\
0 & \mathcal{K}
\end{array}\right], \quad \mathcal{V}=\left[\begin{array}{ll}
\mathcal{V}_{11} & \mathcal{V}_{12} \\
\mathcal{V}_{21} & \mathcal{V}_{22}
\end{array}\right] \\
& \mathcal{V}_{11}=\mathcal{A}+\mathcal{B R C}, \quad \mathcal{V}_{12}=-\mathcal{B} \mathcal{R} \mathcal{B}^{T} \\
& \mathcal{V}_{21}=\mathcal{C}^{T} \mathcal{R C}+\mathcal{Q}_{1}^{T} \mathcal{R} \mathcal{Q}_{1}+\mathcal{Q}_{2}^{T} \mathcal{R} \mathcal{Q}_{2}, \quad \mathcal{V}_{22}=-\left(\mathcal{A}^{T}+\mathcal{C}^{T} \mathcal{R} \mathcal{B}^{T}\right) \\
& \boldsymbol{W}_{11}=\left[\begin{array}{cc}
0 & 0 \\
\mathcal{Q}_{1}^{T} \mathcal{R C}+\mathcal{Q}_{2}^{T} \mathcal{R} \mathcal{Q}_{1} & -\mathcal{Q}_{1}^{T} \mathcal{R} \mathcal{B}^{T}
\end{array}\right] \\
& \boldsymbol{W}_{12}=\left[\begin{array}{cc}
\mathcal{B} \mathcal{R} \mathcal{Q}_{1} & 0 \\
\mathcal{C}^{T} \mathcal{R} \mathcal{Q}_{1}+\mathcal{Q}_{1}^{T} \mathcal{R} \mathcal{Q}_{2} & 0
\end{array}\right] \\
& \boldsymbol{W}_{21}=\left[\begin{array}{cc}
0 & 0 \\
\mathcal{Q}_{2}^{T} \mathcal{R C} & -\left(\mathcal{P}^{T}+\mathcal{Q}_{2}^{T} \boldsymbol{R} \mathcal{B}^{T}\right)
\end{array}\right] \\
& W_{22}=\left[\begin{array}{cc}
\mathcal{P}+\mathcal{B} \mathcal{R} \mathcal{Q}_{2} & 0 \\
\mathcal{C}^{T} \mathcal{R} \mathcal{Q}_{2} & 0
\end{array}\right] \\
& \mathcal{R}=-\left(\mathcal{D}+\mathcal{D}^{T}\right)^{-1}
\end{aligned}
$$

Proof Lemma 3.2.5 shows that an MoC macromodel becomes non-passive when one of the eigenvalues of the matrix $\left[\tilde{\boldsymbol{Y}}(s)+\tilde{\boldsymbol{Y}}^{T}\left(s^{\star}\right)\right]$ assumes negative values for values of $s$ on the $\jmath \omega$-axis. We denote this set of eigenvalues by $\left\{\lambda_{k}(\jmath \omega)\right\}$ and then proceed to prove that the necessary and sufficient condition to have $\lambda_{k}\left(\jmath \omega_{o}\right)=0$ for some value $\omega_{o}$ is that $s_{o}=\jmath \omega_{0}$ satisfies (3.5). The necessity part implies that at $s=\jmath \omega_{o}, \lambda_{k}\left(\jmath \omega_{o}\right)=0$ and therefore the matrix $\left[\tilde{\boldsymbol{Y}}\left(s_{o}\right)+\tilde{\boldsymbol{Y}}^{T}\left(s_{o}^{\star}\right)\right]$ is singular, i.e.,

$$
\left[\boldsymbol{\Pi}\left(s_{o}\right)+\boldsymbol{\Pi}^{T}\left(s_{o}^{\star}\right)\right] \boldsymbol{\eta}=\mathbf{0}
$$


Substituting from (3.3) into (3.15) and using some manipulations yields

$$
\boldsymbol{\eta}=-\boldsymbol{\mathcal { R }}\left(\mathcal{C}+\mathcal{Q}_{1} e^{-s_{o} \tau}+\underline{\mathcal{Q}}_{2} e^{-2 s_{o} \tau}\right) \boldsymbol{r}+\mathcal{R} \mathcal{B}^{T} \boldsymbol{\zeta}
$$

where

$$
\begin{aligned}
\boldsymbol{r} & =-\left(s_{o} \mathcal{K}-\mathcal{A}-\mathcal{P} e^{-2 s_{o} \tau}\right)^{-1} \mathcal{B} \boldsymbol{\eta} \\
\boldsymbol{\zeta} & =\left(-s_{o} \mathcal{K}-\mathcal{A}^{T}-\mathcal{P}^{T} e^{2 s_{o} \tau}\right)^{-1}\left(\mathcal{C}^{T}+e^{s_{o} \tau} \mathcal{Q}_{1}^{T}+e^{2 s_{o} \tau} \mathcal{Q}_{2}^{T}\right) \boldsymbol{\eta} \\
\mathcal{R} & =-\left(\mathcal{D}+\mathcal{D}^{T}\right)^{-1}
\end{aligned}
$$

Rearranging (3.17)-(3.18), we can obtain:

$$
\begin{aligned}
s_{o} \mathcal{K} \boldsymbol{r} & =\left(\mathcal{A}+\mathcal{P} e^{-2 s_{o} \tau}\right) \boldsymbol{r}-\mathcal{B} \boldsymbol{\eta} \\
s_{o} \mathcal{K} \boldsymbol{\zeta} & =-\left(\mathcal{A}^{T}+\mathcal{P}^{T} e^{2 s_{o} \tau}\right) \boldsymbol{\zeta}-\left(\mathcal{C}^{T}+e^{s_{o} \tau} \mathcal{Q}_{1}^{T}+e^{2 s_{o} \tau} \mathcal{Q}_{2}^{T}\right) \boldsymbol{\eta}
\end{aligned}
$$

Substituting (3.16) into (3.20) and (3.21) respectively, and writing in a matrix form yields,

$$
s_{o} \boldsymbol{K} \boldsymbol{\xi}(s)=\boldsymbol{H}\left(s_{o}\right) \boldsymbol{\xi}(s)
$$

where

$$
\begin{aligned}
\boldsymbol{H}\left(s_{o}\right) & =\mathcal{V}+\boldsymbol{W}_{11} e^{s_{o} \tau}+\boldsymbol{W}_{21} e^{2 s_{o} \tau}+\boldsymbol{W}_{12} e^{-s_{o} \tau}+\boldsymbol{W}_{22} e^{-2 s_{o} \tau} \\
\boldsymbol{\xi} & =\left[\begin{array}{ll}
\boldsymbol{r}^{\star} & \boldsymbol{\zeta}^{\star}
\end{array}\right]^{\star}
\end{aligned}
$$

with $\boldsymbol{K}, \mathcal{V}, \boldsymbol{W}_{11}, \boldsymbol{W}_{12}, \boldsymbol{W}_{21}$ and $\boldsymbol{W}_{22}$ being defined as shown by the theorem statement. This proves the necessity condition. 
Proving the sufficiency can be achieved through working in a reverse manner by assuming that $s_{o}=\jmath \omega_{0}$ satisfies

$$
s_{o} \boldsymbol{K} \boldsymbol{\xi}=\boldsymbol{H}\left(s_{o}\right) \boldsymbol{\xi}
$$

and then proceeding to show that $\left[\Pi\left(s_{o}\right)+\Pi^{\star}\left(s_{o}\right)\right]$ is singular. Based on the above analysis, it can be deduced that the absence of an imaginary value for s satisfying (3.5) implies that $\left\{\lambda_{k}(\jmath \omega) \neq 0 \mid \forall \omega \in \mathbb{R}\right\}$ Having $\left\{\lambda_{k}(\jmath \omega) \neq 0 \mid \forall \omega \in \mathbb{R}\right\}$ in MoC macromodels also implies that $\left\{\lambda_{k}(\jmath \omega)\right\}$ remains positive for all values of $\omega$. Thereby making the system passive. This completes the proof.

\subsubsection{Regions of passivity violation}

While Theorem 3.3.1 specifies the necessary and sufficient conditions for a given MoC macromodel to lose passivity, it stops short of locating those regions on the $j \omega$-axis at which the eigenvalues of $\left[\tilde{\boldsymbol{Y}}(s)+\tilde{\boldsymbol{Y}}^{T}\left(s^{\star}\right)\right]$ become negative, and render the macromodel non-passive. The following theorem is aimed at addressing this issue.

Theorem 3.3.2 A non-passive MoC macromodel has the eigenvalue(s) of $\left[\tilde{\boldsymbol{Y}}(s)+\tilde{\boldsymbol{Y}}^{T}\left(s^{\star}\right)\right]$ vanishing at those points of $s$ on the jw-axis that satisfy (3.5).

Proof Proof of Theorem 3.3.2 follows easily from the proof of Theorem 3.3.1, in that if $s_{o}$ is an imaginary value satisfying (3.5), then the matrix $\left[\tilde{\boldsymbol{Y}}\left(s_{o}\right)+\tilde{\boldsymbol{Y}}^{T}\left(s_{o}^{\star}\right)\right]$ is singular and the system becomes non-passive.

Theorem 3.3.1 and 3.3.2 established two essential facts which can be summarized as follows:

1. If it is possible to compute the set of all generalized eigenvalues of $s$ satisfying the FD-GEP in (3.5) and this set does not contain any purely imaginary values, then one can conclude that (3.1) is satisfied and the macromodel is passive. 
2. On the other hand, if this set of solutions contains one or more pure imaginary generalized eigenvalues, then the system becomes non-passive and the passivity violation regions (PVRs) are delimited by those imaginary eigenvalues.

The challenging issue that remains to be addressed so far is related to solving the FDGEP in (3.5) developed in Theorem 3.3.1. Given the fact that the associated matrix pencil $(\boldsymbol{H}(s), \boldsymbol{K})$ is not a constant pencil, classical approaches of handling generalized eigenvalue problems can not be invoked to solve this problem. This issue is addressed in the following section.

\subsection{Computational considerations}

The main issue addressed in this section is basically finding those values for $s \mid \Re(s)=0$ satisfying the FD-GEP in (3.5). In fact, solving this problem is compounded by the fact that it cannot be formulated as a polynomial function of $s$ since the associated matrix pencil is a transcendental function of $s$. The following theorem presents the key point in approaching this problem.

Theorem 3.4.3 Let $\left\{\sigma_{k}(s)\right\}$ be the set of generalized eigenvalues of the matrix pencil $(\boldsymbol{H}(s), \boldsymbol{K})$ for any value $s \in \mathbb{C}$, i.e.,

$$
\sigma_{k}(s) \boldsymbol{K} \boldsymbol{\xi}_{k}=\boldsymbol{H}(s) \boldsymbol{\xi}_{k}
$$

with $\boldsymbol{\xi}_{k}$ being the corresponding eigenvector. Hence, $\sigma_{k}(s)$ is periodical function of $s$ for all values of s lying on the imaginary axis $(s=\jmath \omega, \omega \in \mathbb{R})$, with a period of $\frac{2 \pi}{\tau}$.

Proof Proof of Theorem 3.4.3 can be deduced by noting that $\boldsymbol{H}(s)$ as defined by (3.6), is composed of a summation of constant matrices that are independent of $s$, while the summation coefficients are given by $e^{n s \tau}, n=0, \pm 1, \pm 2$. Therefore, if $s$ is restricted to purely 
imaginary values, i.e. $s=\jmath \omega$, then the summation coefficients become periodical functions with a fundamental period of $\frac{2 \pi}{\tau}$. Hence, $\boldsymbol{H}(s)$ is also periodical with the same fundamental period. Since $\boldsymbol{K}$ is a constant matrix independent of $s$, then the set of generalized eigenvalues associated with the matrix pencil $(\boldsymbol{H}(s), \boldsymbol{K})$ can also be represented by periodical function of $s$ with a fundamental period of $\frac{2 \pi}{\tau}$.

Note that in the statement of Theorem 3.4.3, the notation of having $\sigma_{k}(s)$ explicitly formulated as a function of $s$ is used to emphasize that the generalized eigenvalue is $s$ dependent. Based on Theorem 3.4.3, one can write that

$$
\sigma_{k}(s)=\sigma_{k}\left(s+j \frac{2 n \pi}{\tau}\right) \quad n=0, \pm 1, \pm 2, \ldots
$$

It can be concluded from Theorem 3.4.3 that the objective of finding the imaginary values for $s$ satisfying (3.5) can be formulated alternatively to seek values of $s$ that satisfy,

$$
\sigma_{k}(s)=s \quad s \in[\jmath 0, \jmath \infty]
$$

To solve (3.27) for pure imaginary values $s$, one can take advantage of the fact that $\sigma_{k}(s)$ is periodic in $s$. A two-phase procedure is adopted to approach this problem.

\subsubsection{Phase I: Estimation of $\Re\left[\sigma_{k}(s)\right]$}

In this phase, the set of generalized eigenvalues $\left\{\sigma_{k}(s)\right\}$ is computed by solving the FDGEP in (3.25) for a single period, i.e., for values of $s \in\left[\jmath 0, \jmath \frac{2 \pi}{r}\right)$. Since the set $\left\{\sigma_{k}(s)\right\}$ is periodic for values of $s$ on the $\jmath \omega$-axis with a period of $\frac{2 \pi}{\tau}$, computation of $\left\{\sigma_{k}(s)\right\}$ within this interval is intended to provide a rough estimate for the variation of $\left\{\sigma_{k}(s)\right\}$ on the entire $\jmath \omega$-axis. Since our goal is to find values of $s$ on this axis (i.e., $\Re(s)=0$ ) satisfying (3.27), we should be looking for those eigenvalues in $\left\{\sigma_{k}(s)\right\}$ that have diminishing real parts. If the outcome of this computation shows that all $\left|\Re\left\{\sigma_{k}(s)\right\}\right|>\epsilon$ for all $s \in\left[\jmath 0, j \frac{2 \pi}{\tau}\right)$ where $\epsilon$ 
is some positive number, then $\left\{\sigma_{k}(s)\right\}$ can not assume a pure imaginary value for all values of $s$ on the imaginary axis. Consequently, the system is passive and no need to proceed with the next phase. This conclusion is based on the fact that $\left\{\sigma_{k}(s)\right\}$ is periodic on the imaginary axis, and if there is no imaginary value of $\left\{\sigma_{k}(s)\right\}$ satisfying (3.25) within one period, then no other imaginary value can even exist to satisfy this condition. On the other hand, if the outcome of this phase shows that the real part of $\left\{\sigma_{k}(s)\right\}$ becomes close or equal to zero for some value of $s_{\epsilon}=\jmath \omega_{\epsilon}$, then this signals a potential loss of non-negative definiteness for $\Pi(s)$. A subsequent phase therefore needs to be initiated to confirm or detect the exact point at which PR-ness violation occurs.

\subsubsection{Phase II: Locating the PR-ness violations}

The previous phase can be considered as a preprocessing stage to identify those eigenvalues with diminishing real-parts. Those eigenvalues represent the starting point for Phase II. More specifically, the entry to this phase is a set of two-point pairs on the imaginary axis, $\left\{\omega_{1}\right.$ and $\left.\omega_{2}\right\}$, such that

$$
\Re\left\{\sigma_{k}\left(\jmath \omega_{1}\right)\right\} \leq 0 \text { and } \Re\left\{\sigma_{k}\left(\jmath \omega_{2}\right)\right\} \geq 0,
$$

or

$$
\Re\left\{\sigma_{k}\left(\jmath \omega_{1}\right)\right\} \geq 0 \text { and } \Re\left\{\sigma_{k}\left(\jmath \omega_{2}\right)\right\} \leq 0
$$

and the objective is to locate the point $\omega_{o}$ where $\omega_{1} \leq \omega_{o} \leq \omega_{2}$ such that

$$
\Re\left\{\sigma_{k}\left(\jmath \omega_{o}\right)\right\}=0,
$$

and

$$
\Im\left\{\sigma_{k}\left(\jmath \omega_{o}\right)\right\}=\omega_{o}
$$


For this purpose, each exponential function in (3.5) is approximated with a Chebyshev polynomial of the first kind within the interval of $\left[\jmath \omega_{1}, \jmath \omega_{2}\right]$ [89]. The order of this approximation is usually very small since the interval of $\left[\jmath \omega_{1}, \jmath \omega_{2}\right]$ is small and Chebyshev polynomial has an optimal feature in approximating a function within an specific interval. As a result, the FD-GEP is transformed into a generalized polynomial eigenvalue problem (GPEP), which is well-known $[90,91]$ and can be solved easily for pure imaginary values for $s$. This idea is substantiated by the following lemma.

Lemma 3.4.6 let each of $e^{s \tau}, e^{-s \tau}, e^{2 s \tau}$ and $e^{-2 s \tau}$ be approximated by a $q^{\text {th }}$-order Chebyshev polynomial of the first kind, $T_{h}(s)$, for the interval of $\left[\jmath \omega_{1}, \jmath \omega_{2}\right]$, i.e.,

$$
\begin{aligned}
e^{s \tau} & \approx \sum_{h=0}^{q} a_{h}^{(11)} T_{h}(\hat{s}) & e^{-s \tau} & \approx \sum_{h=0}^{q} a_{h}^{(12)} T_{h}(\hat{s}) \\
e^{2 s \tau} & \approx \sum_{h=0}^{q} a_{h}^{(21)} T_{h}(\hat{s}) & e^{-2 s \tau} & \approx \sum_{h=0}^{q} a_{h}^{(22)} T_{h}(\hat{s})
\end{aligned}
$$

where

$$
\hat{s}=2 \frac{s-\jmath \omega_{1}}{\jmath \omega_{2}-\jmath \omega_{1}}-1 \in[-1,1]
$$

and $\left\{a_{h}^{(i j)} \mid i, j=1,2, \quad h=0, \ldots, q\right\}$ are coefficients for the Chebyshev polynomials. Then values of $s \in\left[\jmath \omega_{1}, \jmath \omega_{2}\right]$ satisfying the FD-GEP in (3.5) can be approximated from

$$
s=\frac{\jmath \omega_{2}-\jmath \omega_{1}}{2} \hat{s}+\frac{\jmath \omega_{2}+\jmath \omega_{1}}{2}
$$

where $\hat{s}$ is the solution of the following generalized polynomial eigenvalue problem,

$$
\sum_{k=0}^{q}\left(\boldsymbol{\Upsilon}_{k} s^{k}\right) \boldsymbol{\xi}=\mathbf{0}
$$

and $\Upsilon_{k}$ is defined in the following proof.

Proof It is straightforward to conclude that (3.30) is true from (3.29). Hence the only thing we need to prove is that $\hat{s}$ satisfies the GPEP in (3.31). To proceed with the proof, we 
substitute from (3.28) and (3.30) into (3.5) to obtain

$$
\sum_{h=0}^{q}\left(\boldsymbol{\Psi}_{h} T_{h}(\hat{s})\right) \boldsymbol{\xi}=\mathbf{0}
$$

where

$$
\begin{aligned}
& \boldsymbol{\Psi}_{0}=a_{0}^{(11)} \boldsymbol{W}_{11}+a_{0}^{(12)} \boldsymbol{W}_{12}+a_{0}^{(21)} \boldsymbol{W}_{21}+a_{0}^{(22)} \boldsymbol{W}_{22}-\frac{\jmath \omega_{2}+\jmath \omega_{1}}{2} \boldsymbol{K}+\mathcal{V}( \\
& \boldsymbol{\Psi}_{1}=a_{1}^{(11)} \boldsymbol{W}_{11}+a_{1}^{(12)} \boldsymbol{W}_{12}+a_{1}^{(21)} \boldsymbol{W}_{21}+a_{1}^{(22)} \boldsymbol{W}_{22}-\frac{\jmath \omega_{2}-\jmath \omega_{1}}{2} \boldsymbol{K} \\
& \boldsymbol{\Psi}_{h}=a_{h}^{(11)} \boldsymbol{W}_{11}+a_{h}^{(12)} \boldsymbol{W}_{12}+a_{h}^{(21)} \boldsymbol{W}_{21}+a_{h}^{(22)} \boldsymbol{W}_{22} \quad h \geq 2
\end{aligned}
$$

(3.32) can be easily transformed into the following polynomial eigenvalue problem in $\hat{s}$ by substituting each Chebyshev function $T_{h}(\hat{s})$ with its associated regular polynomial, i.e., $T_{h}(\hat{s})=\sum_{k=0}^{q} b_{h}^{k} \hat{s}^{k}$ where $b_{h}^{k}$ 's are constant coefficients that can be obtained as shown in [89].

$$
\sum_{k=0}^{q}\left(\boldsymbol{\Upsilon}_{k} \hat{s}^{k}\right) \boldsymbol{\xi}=\mathbf{0}
$$

where

$$
\Upsilon_{k}=\sum_{h=0}^{q} \Psi_{h} b_{h}^{k}
$$

Lemma 3.4.6 states that if there exists a purely imaginary value for $s$, say $s_{o}=\jmath \omega_{o}$, that satisfies (3.30) and, if $\omega_{o}$ lies within the frequency interval, $\left[\omega_{1}, \omega_{2}\right]$, then $s_{o}$ is also a purely imaginary solution of the FD-GEP associated with the matrix pencil $\left(\boldsymbol{H}\left(s_{o}\right), \boldsymbol{K}\right)$ of (3.5). Repeating the approximation for all the frequency intervals that were obtained from the first phase, where loss of passivity can potentially occur, and solving (3.31) for imaginary $s$ for each approximation, enables finding all the imaginary solutions to the FD-GEP in (3.5). 


\subsection{Computational complexity}

The proposed approach has been implemented in MATLAB ${ }^{\circledR}$ to verify the passivity of MoC-based macromodels. This section lays out the main computational complexity involved in the implementation. The essential part of the required computations is needed in performing eigenvalue decomposition of the matrix $\boldsymbol{H}(s)$ whose size depends on the order of rational approximation for $Y_{c}(s)$ and $P(s)$. More specifically, for a rational approximation with $q^{P}$ poles for $P(s)$ and $q^{Y}$ poles for $Y_{c}(s), \boldsymbol{H}(s)$ becomes a matrix in $\mathbb{C}^{N \times N}$, where $N=4\left(2 q^{P}+q^{Y}\right)$. Once a potential passivity violation has been identified, a polynomial eigenvalue problem is invoked to obtain more accurate values for the frequency points at which the violation occurred. The size of the resulting eigenvalue problem becomes $q N$, where $q$ is the highest order of the Chebyshev polynomials used in approximating the exponential functions.

\subsection{Numerical examples}

The proposed passivity verification algorithm was implemented to check for the passivity of TL macromodels derived using MoC. Three different macromodels have been considered for that purpose. For notational convenience, $\left\{\lambda_{k}(\jmath \omega)\right\}$ is used to denote the set of the eigenvalues of the matrix $\left[\tilde{\boldsymbol{Y}}(s)+\tilde{\boldsymbol{Y}}^{T}\left(s^{\star}\right)\right]$ computed for values of $s$ taken on the $j \omega$-axis.

\subsubsection{Example 1}

A 4cm two-conductor TL with frequency-independent PUL parameters given in (3.38) was considered in this example. An MoC model was generated by approximating the characteristic admittance and the delayless propagation factor $\left(Y_{c}(s)\right.$ and $\left.P(s)\right)$ with rational 
approximating functions of orders $(2 / 2)$ and $(2 / 3)$ respectively. Figure 3.2 shows a comparison between the MoC-based macromodel's admittance matrix $(\tilde{\boldsymbol{Y}}(s))$ and that of the original TL network.

$$
R=0.014 k \Omega / m, \quad L=0.63 \mu H / m, \quad G=0.001 \mathrm{mS} / \mathrm{m}, \quad C=110 \mathrm{pf} / \mathrm{m}
$$

The proposed algorithm was then implemented to check the passivity of the MoC macro-

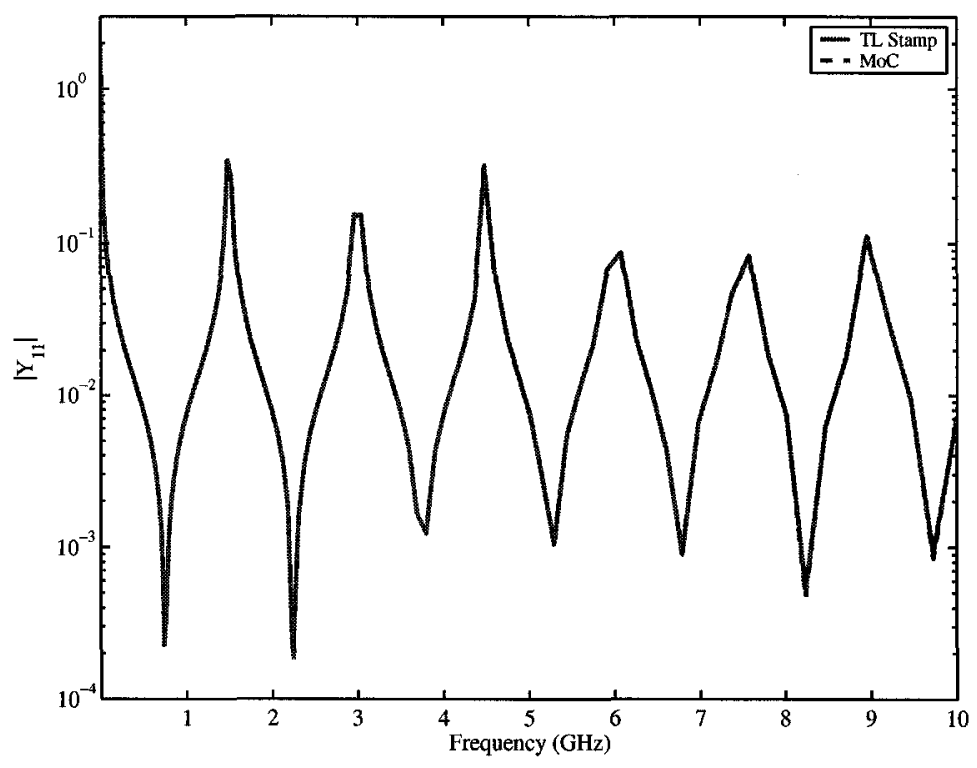

Figure 3.2: Comparison of admittance matrices between the MoC model and the original TL network (Example 1).

model. An ADDEs was first constructed as shown in Proposition 3.3.1, and the corresponding set of generalized eigenvalues $\left\{\sigma_{k}(s)\right\}$ were computed for values of $s$ within one period on the $\jmath \omega$-axis, i.e., for $s \in\left[0, \jmath \frac{2 \pi}{\tau}\right)$ where $\tau$ is the delay of the line estimated by taking it as indicated earlier equal $\sqrt{C L}=0.333 \mathrm{~ns}$. Subsequently, it was observed that the real part of some of those eigenvalues vanish for values of $s$ falling in the range $s \in\left[\jmath 0, \jmath 0.99917 \times 10^{8}\right]$, indicating that a potential loss of passivity could occur. Phase II was then initiated where 
each exponential function was approximated by a $4^{t h}$-order Chebyshev polynomial approximation within the above band of frequencies resulting in one GPEP as has been illustrated by Lemma 3.4.6. The GPEP then yielded four purely imaginary eigenvalues at $s=\jmath 1.9371 \times 10^{4}, \jmath 1.5347 \times 10^{5}, \jmath 3.9534 \times 10^{6}$ and $\jmath 3.7336 \times 10^{7}$. According to the results of Theorem 3.3.2, these imaginary eigenvalues predict that the MoC model loses passivity in the regions delimited by those values. In other words, some eigenvalue(s) of $\left[\tilde{\boldsymbol{Y}}(s)+\tilde{\boldsymbol{Y}}^{T}\left(s^{\star}\right)\right]$ cross from positive to negative (or negative to positive) values at exactly those four values of $s$ on the imaginary axis.

To verify the validity of the results predicted by the algorithm, $\left\{\lambda_{k}(\jmath \omega)\right\}$ were computed for values of $s$ on the $\jmath \omega$-axis up to a very high value. Figure 3.3(a) shows the values that $\lambda_{1}(\jmath \omega)$ takes as $\omega$ is changed from 0 to $62.8 \times 10^{9}$ radian. Although, it may seem on a first glance that $\lambda_{1}(\jmath \omega)$ remain positive for all possible values of $\omega$, closer inspection reveals that $\lambda_{1}(\jmath \omega)$ becomes negative towards small values of $\omega$. Figure 3.3(b) depicts an enlarged picture for $\lambda_{1}(\jmath \omega)$ at small values of $\omega$. Also shown in the figure are the values $\omega$ at which $\lambda_{1}(\jmath \omega)$ vanishes. Those values are the points at which the model loses passivity, and, as can be directly observed, are in very good agreement with the results predicted by the proposed algorithm.

To provide further insight into the numerical accuracy of the above results, writing the eigenvalue $\left\{\lambda_{k}(\jmath \omega)\right\}$ in an analytical close-form expression in $\omega$, then employing the iterative nonlinear solver, Newton-Raphson (N-R) [92], to find the points at which those values vanish. This process resembles a root finding process in which an analytical nonlinear function is represented by $\left\{\lambda_{k}(\jmath \omega)\right\}$ and the independent variable is given by $\omega$, and the goal is to find the values $\omega_{o}$ at which $\lambda_{k}\left(\jmath \omega_{o}\right)=0$. It is worth mentioning that this approach is applicable only to the special case of a two-conductor TL, where the analytical expression for $\left\{\lambda_{k}(\jmath \omega)\right\}$ is available, and requires some prior information that can be used as a seed 


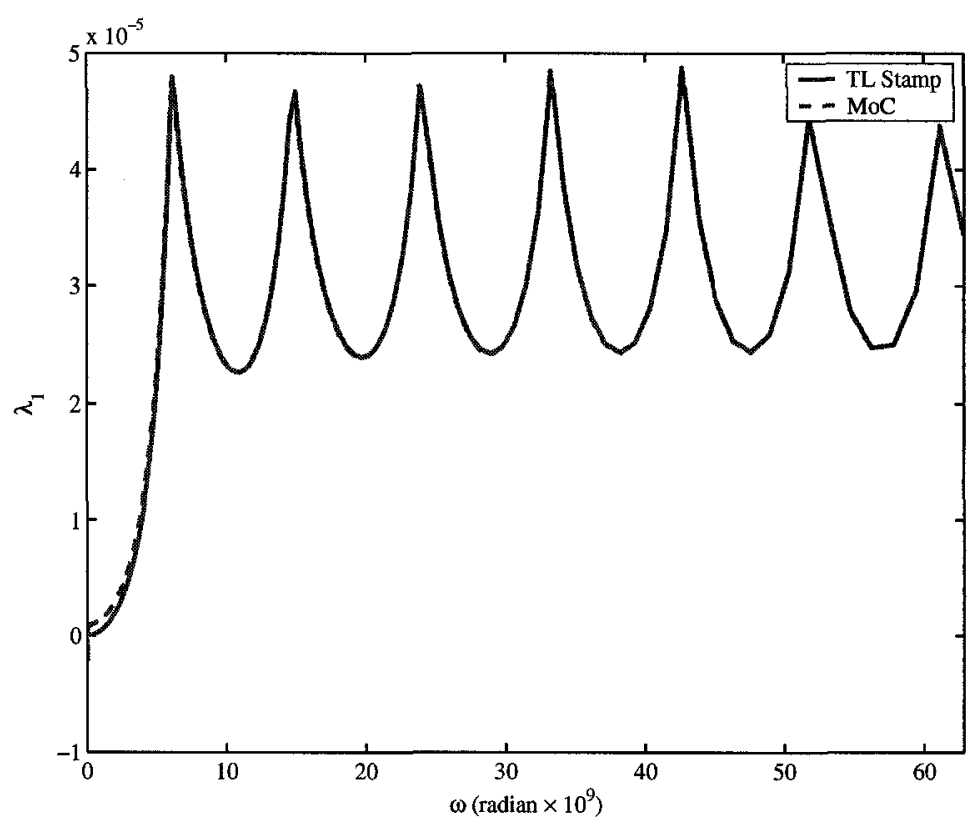

(a) $\lambda_{1}(\jmath \omega)$.

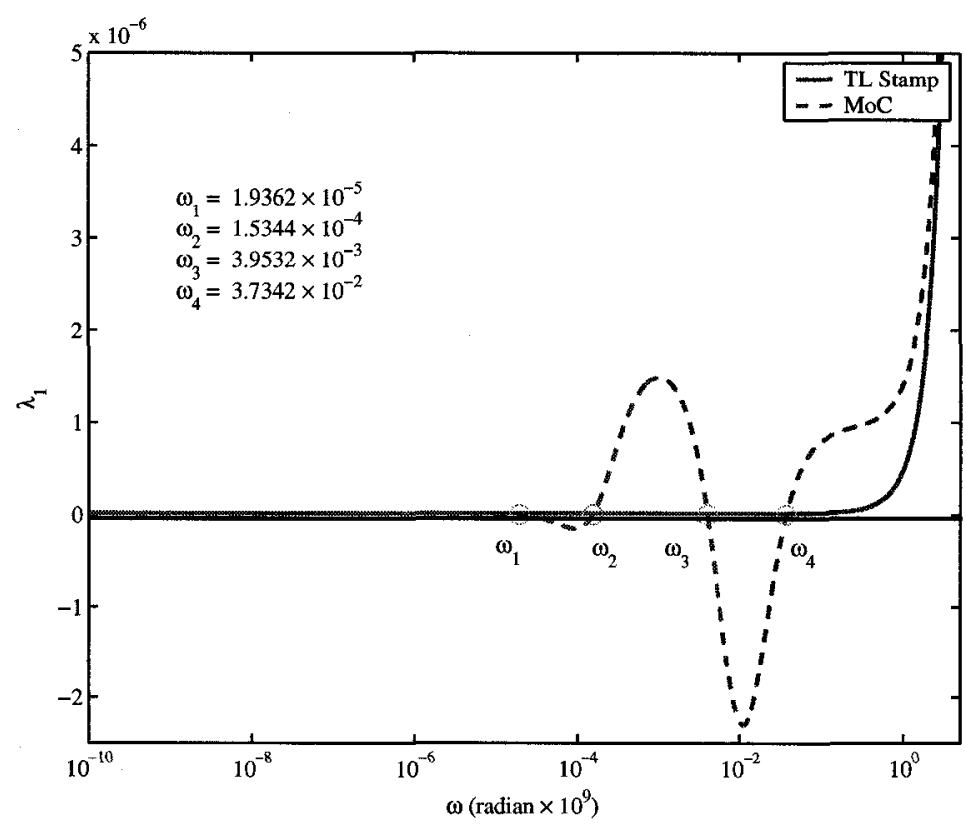

(b) Zoom of $\lambda_{1}(\jmath \omega)$ showing negative values.

Figure 3.3: $\lambda_{1}(\jmath \omega)$ (Example 1). 
to guide the convergence of the iterative solver. Although this seed point can be obtained through sweeping $s$ on the imaginary axis and computing the corresponding eigenvalues to detect regions that are close to zero value, it remains impractical to determine all the zero crossings for the eigenvalues.

Table 3.1 lists the results obtained in the last few iterations, before convergence, of using the N-R method to obtain the exact values of $\omega$ at which $\lambda_{1}(\jmath \omega)=0$. The first column in the table reflects the value of solution $\omega$ as it approaches the root value satisfying $\lambda_{1}(\jmath \omega)=0$, while the second column reflects the corresponding value of $\lambda_{1}(\jmath \omega)$. The numerical results in the table show a very good agreement with those obtained from the proposed algorithm.

Table 3.1: Results of the last few N-R iterations before convergence to four zeroes of $\lambda_{1}(\jmath \omega)$. The 4 zero points are denoted by $\omega_{1}, \omega_{2}, \omega_{3}$ and $\omega_{4}$ and are described in each section of the table (Example 1).

\begin{tabular}{|c|c|c|}
\hline Iteration & $\omega_{1}\left(\right.$ radian $\left.\times 10^{9}\right)$ & $\lambda_{1}\left(\jmath \omega_{1}\right)$ \\
\hline \hline $1^{\text {st }}$ & $1.9628 \mathrm{e}-005$ & $-4.8624 \mathrm{e}-007$ \\
\hline $2^{\text {nd }}$ & $1.9365 \mathrm{e}-005$ & $-2.6871 \mathrm{e}-009$ \\
\hline $3^{\text {rd }}$ & $1.9363 \mathrm{e}-005$ & $-8.4362 \mathrm{e}-014$ \\
\hline \hline & $\omega_{2}\left(\right.$ radian $\left.\times 10^{9}\right)$ & $\lambda_{1}\left(\jmath \omega_{2}\right)$ \\
\hline \hline $1^{\text {st }}$ & $1.5391 \mathrm{e}-004$ & $1.9237 \mathrm{e}-006$ \\
\hline $2^{\text {nd }}$ & $1.5347 \mathrm{e}-004$ & $3.9381 \mathrm{e}-009$ \\
\hline $3^{\text {rd }}$ & $1.5347 \mathrm{e}-004$ & $1.6845 \mathrm{e}-014$ \\
\hline \hline & $\omega_{3}\left(\right.$ radian $\left.\times 10^{9}\right)$ & $\lambda_{1}\left(\jmath \omega_{3}\right)$ \\
\hline \hline $1^{\text {st }}$ & 0.0039869 & $-2.1848 \mathrm{e}-005$ \\
\hline $2^{\text {nd }}$ & 0.0039535 & $1.0103 \mathrm{e}-008$ \\
\hline $3^{\text {rd }}$ & 0.0039535 & $1.9196 \mathrm{e}-015$ \\
\hline \hline & $\omega_{4}\left(\right.$ radian $\left.\times 10^{9}\right)$ & $\lambda_{1}\left(\jmath \omega_{4}\right)$ \\
\hline \hline $1^{\text {st }}$ & 0.0372943 & $-1.8188 \mathrm{e}-006$ \\
\hline $2^{\text {nd }}$ & 0.0373369 & $-2.4171 \mathrm{e}-009$ \\
\hline $3^{\text {rd }}$ & 0.0373369 & $-4.2658 \mathrm{e}-015$ \\
\hline
\end{tabular}




\subsubsection{Example 2}

A relatively long $(10 \mathrm{~m})$ coaxial cable TL with frequency-dependent PUL parameters is considered for this example. The variation of the PUL parameters versus frequency can be found in [85]. MoC was used to construct a model and the resulting macromodel was then considered for passivity verification using the proposed algorithm. Figure 3.4 shows the approximation of $Y_{c}(s)$ and $P(s)$ and the comparison of admittance matrix between MoC model and TL stamp is shown in Figure 3.5. Even though the MoC model is in a very good match with the TL stamp as shown in Figure 3.5, applying the proposed algorithm to the MoC model showed that there are four purely imaginary eigenvalues satisfying the GPEP. Those values occur at

$$
s=\jmath 2.6958 \times 10^{3}, \quad \jmath 1.6468 \times 10^{5}, \quad \jmath 3.5743 \times 10^{5}, \quad \text { and } \jmath 1.9579 \times 10^{6}
$$

Based on the theory developed in this paper, this fact indicates that one or more eigenvalue of $\left[\tilde{\boldsymbol{Y}}(\jmath \omega)+\tilde{\boldsymbol{Y}}^{T}(-\jmath \omega)\right]$ becomes zero at values $s$ equal to those given in (3.39).

To demonstrate the validity of the results thus obtained, $\left\{\lambda_{k}(\jmath \omega)\right\}$ have been computed and plotted in Figure 3.6 where $\lambda_{1}(\jmath \omega)$ shows the passivity violation points corroborating what has been obtained earlier in (3.39).

\subsubsection{Example 3}

In this example, another $10 \mathrm{~cm}$ lossy two-conductor TL was considered for the passivity verification algorithm. The PUL parameter is listed in (3.40)

$$
R=3.36 \Omega / m, L=0.2669 \mu H / m, G=4.758 e-8 S / m, C=90.18 p f / m
$$




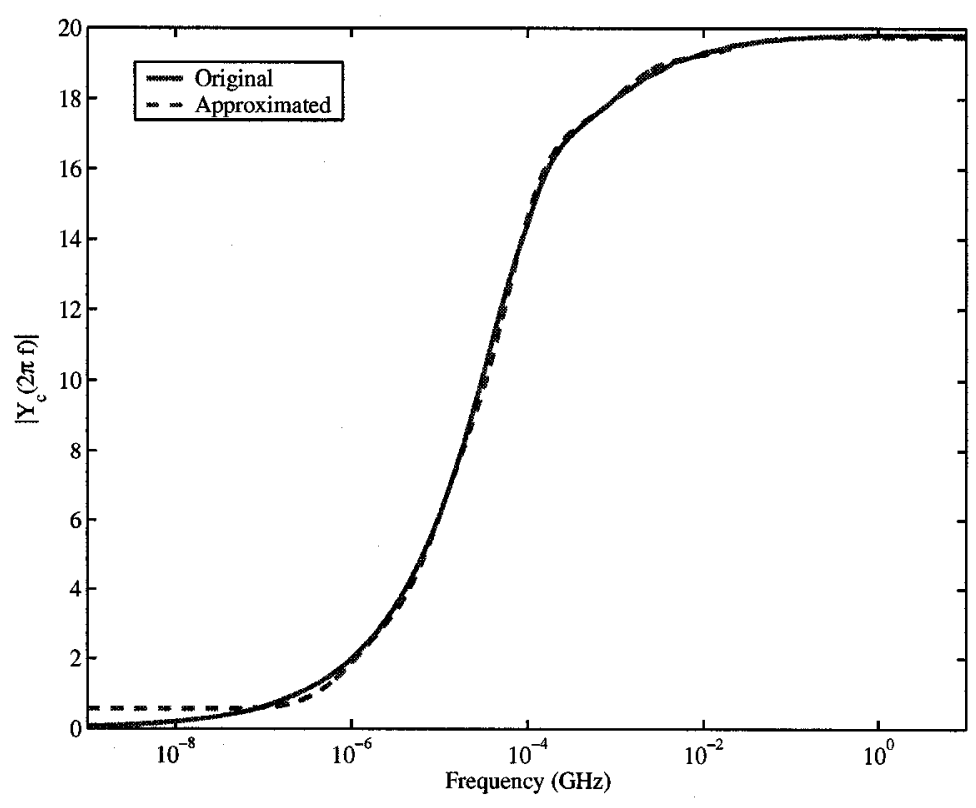

(a) $\tilde{Y}_{c}(s)$.

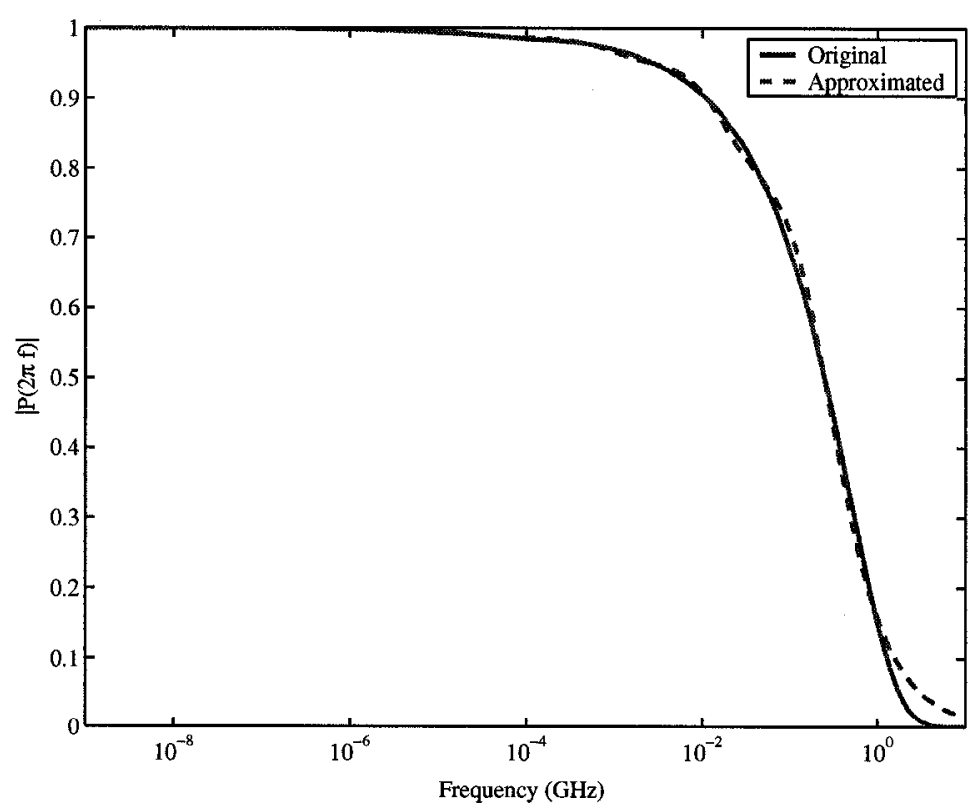

(b) $\tilde{P}(s)$.

Figure 3.4: Comparison of approximation and original data for $Y_{c}(s)$ and $P(s)$ (Example 2). 


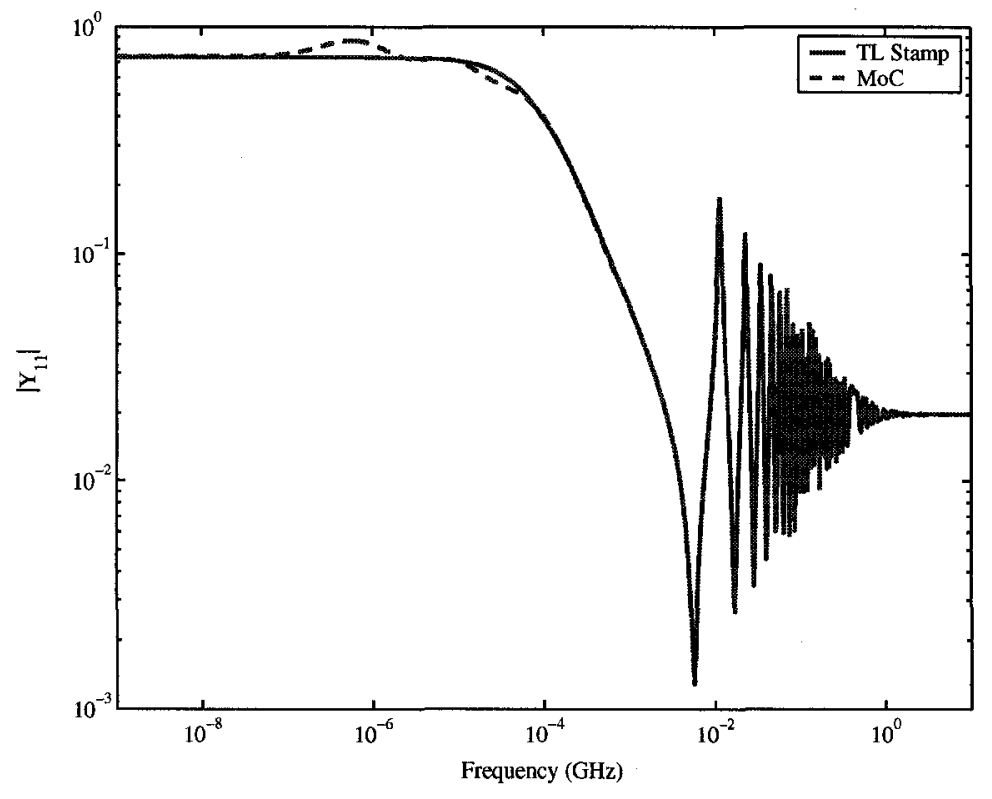

Figure 3.5: Comparison of admittance matrices between the MoC model and the original TL network (Example 2).

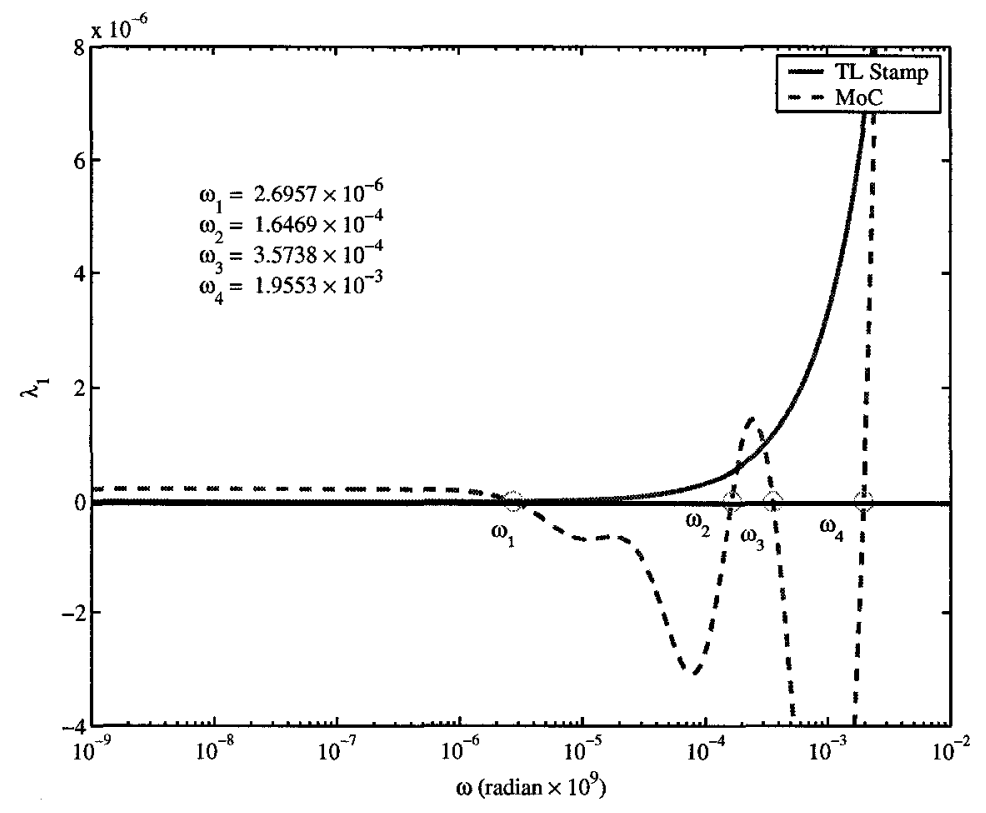

Figure 3.6: Zoom of $\lambda_{1}(\jmath \omega)$ at small $\omega$ (Example 2). 
An MoC-based macromodel was constructed, where one element of its admittance matrix is shown in Figure 3.7(a). Applying the proposed algorithm on the MoC model showed that no purely imaginary eigenvalues for the GPEP exist. This result then confirmed that the model is passive. To corroborate the result obtained, $\left\{\lambda_{k}(\jmath \omega)\right\}$ were computed and plotted in Figure 3.7(b). As can be observed from the figure, those eigenvalues remain positive for very high frequency indicating that the model is passive. This is in agreement with the conclusion drawn from implementing the proposed algorithm.

Table 3.2 summarizes the CPU time needed to run the proposed approach and validate the passivity of the TL's in the three examples. The CPU time reported in this table is provided through a MATLAB ${ }^{\circledR}$ implementation on Windows XP platform running on a PC Pentium IV $(1.5 \mathrm{GHz})$ processor. The first column in the table shows the time needed to validate the passivity. The second column provides the time needed to locate the passivity violation regions.

Table 3.2: CPU time comparison (seconds).

\begin{tabular}{|c|c|c|}
\hline Examples & CPU Time $^{1}$ & CPU Time $^{2}$ \\
\hline \hline Example 1 & 0.2 & 0.55 \\
\hline Example 2 & 2.2 & 2.2 \\
\hline Example 3 & 1.1 & - \\
\hline
\end{tabular}

1. time in checking the passivity of the model.

2 . time in detecting the passivity violation points. 


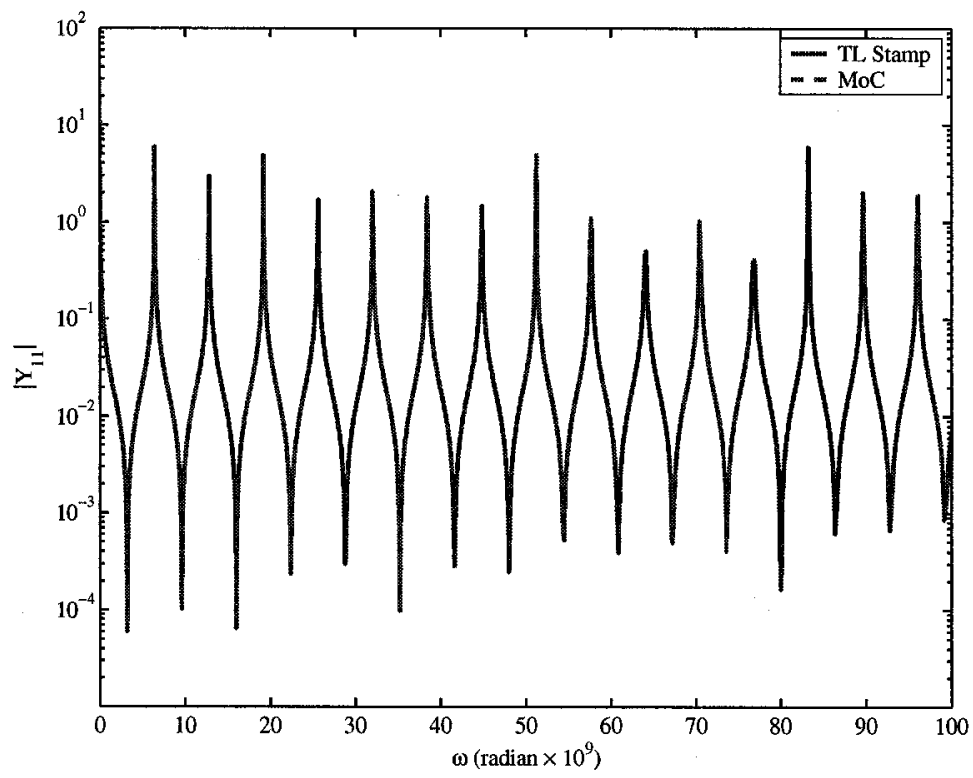

(a) Comparison of admittance matrix between the MoC model and the original TL network.

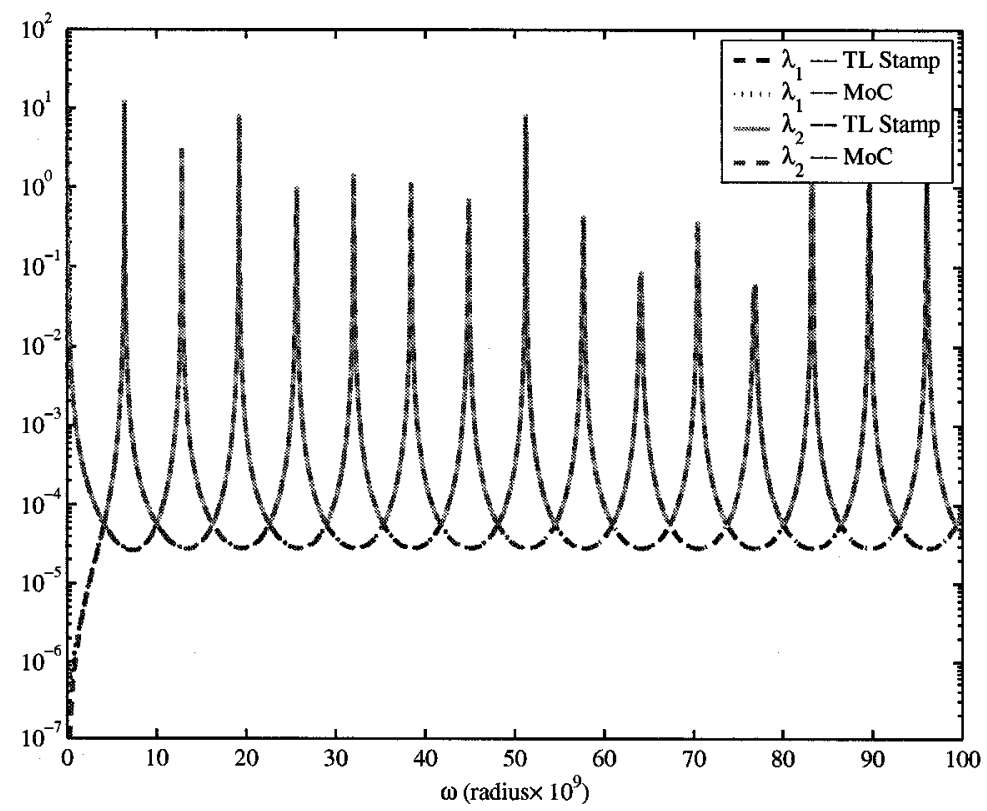

(b) Eigenvalus of $\tilde{\boldsymbol{Y}}(\jmath \omega)+\tilde{\boldsymbol{Y}}^{T}(-\jmath \omega)$.

Figure 3.7: Numerical results for Example 3. 


\section{Chapter 4}

\section{Passivity Verification in Delay-Based Macromodels of Multi-conductor Transmission Lines}

\subsection{Introduction}

Chapter 3 presented a new theory that describes the necessary and sufficient conditions for passivity verification of non-passive MoC-based macromodels of two-conductor electrical interconnects. Based on the theory, an algebraic test is proposed to check the passivity of the macromodel. In this chapter, this idea is further generalized to address the passivity verification problem for multi-conductor electrical interconnects [31].

Development of the proposed approach proceeds by proving that a MoC-based macromodel can be decomposed into two submodels, which can be represented in the timedomain by systems of delay differential equations. The necessary and sufficient conditions for the positive realness of the transfer function in each submodel is then derived. The proposed theory demonstrates that the passivity of the MoC macromodel can be verified by solving an eigenvalue problem similar to that developed in Chapter 3 for the two-conductor case. 
The rest of this chapter is organized as follows. Section 4.2 presents a basic assumption in $\mathrm{MoC}$ macromodels in order to facilitate the presentation of the proposed approach. Section 4.3 lays out the theoretical basis behind the proposed approach. Section 4.4 presents the computational implementation procedure to verify the passivity of the MoC macromodel. Section 4.5 presents some numerical examples to demonstrate the validity of the proposed approach.

\subsection{Passivity of the MoC-based macromodels}

The goal of this chapter is to derive the necessary and sufficient conditions for a MoCbased macromodel of multi-conductor lossy TL to be passive. It is well known that such a model is passive if and only if its admittance matrix satisfies three conditions of PRness. Given the difficulty of using those conditions directly to verify the passivity, some simplified conditions were derived in Chapter 3 for the MoC-based macromodels of the two-conductor TLs. The following lemma generalizes this result further to the case of MTLs. The admittance matrix of the macromodel in this case is given by $\tilde{\boldsymbol{Y}}(s)$ in (2.13).

Lemma 4.2.7 Assume that the MoC-based model is asymptotically stable, (i.e., $\tilde{\boldsymbol{Y}}(s)$ has no singular points for $\Re(s) \geq 0$ ), and that both of $\tilde{\boldsymbol{Y}}_{c}(s)$ and $\tilde{\boldsymbol{P}}(s)$ do not have poles in the right-half plane of the s-domain with $\tilde{\boldsymbol{P}}(s)$ being a strict rational function. Then $\tilde{\boldsymbol{Y}}(s)$ satisfies the first two conditions of being a PR matrix. Furthermore, the third condition is satisfied in $\Re(s)>0$ if it is satisfied on the imaginary axis $(\Re(s)=0)$. In other words, the third condition for PR-ness can be replaced by

$$
\boldsymbol{z}^{\star T}\left[\tilde{\boldsymbol{Y}}^{T}(-\jmath \omega)+\tilde{\boldsymbol{Y}}(\jmath \omega)\right] \boldsymbol{z} \geq 0 \quad \forall \omega \in \mathbb{R} \text { and } \forall \boldsymbol{z} \in \mathbb{C}^{2 m}
$$


The proof of Lemma 4.2.7 is similar to the proof of Lemma 3.2.5 in Chapter 3. Hence it is omitted here for brevity. While the condition in (4.1) may be viewed as a considerable simplification towards a simple passivity test, its implementation remains a formidable task since it requires testing an infinite number of frequency points on the $\jmath \omega$-axis. The next section develops a theoretical framework addressing this issue.

\subsection{Theoretical basis}

The first step in developing the proposed theory demonstrates that the passivity of a MoC macromodel can be verified via verifying the passivity of two submodels. This point is established in Section 4.3.1 and presents a key idea in the following derivations. Section 4.3.2 then demonstrates that each one of those submodels can be realized by three dynamical systems whose structures are described by systems of differential equations with some delay elements in the outputs. Section 4.3.3 derives the main result of this section which prescribes the necessary and sufficient conditions for the passivity of each submodel. Finally, Section 4.3.4 presents a theorem that locates the exact passivity violation regions of a nonpassive MoC macromodel.

\subsubsection{Decomposition of MoC macromodels}

The idea of checking the passivity of a MoC macromodel through decomposing it into two submodels is established using the following lemma.

Lemma 4.3.8 If $\boldsymbol{Y}_{1}(s)$ and $\boldsymbol{Y}_{2}(s)$ denote the admittance matrices given by,

$$
\begin{aligned}
& \boldsymbol{Y}_{1}(s)=\left(\boldsymbol{I}_{m}+\boldsymbol{M} e^{-s \tau} \tilde{\boldsymbol{P}}(s) \boldsymbol{M}^{-1}\right)^{-1}\left(\boldsymbol{I}_{m}-\boldsymbol{M} e^{-s \tau} \tilde{\boldsymbol{P}}(s) \boldsymbol{M}^{-1}\right) \tilde{\boldsymbol{Y}}_{c}(s) \\
& \boldsymbol{Y}_{2}(s)=\left(\boldsymbol{I}_{m}-\boldsymbol{M} e^{-s \tau} \tilde{\boldsymbol{P}}(s) \boldsymbol{M}^{-1}\right)^{-1}\left(\boldsymbol{I}_{m}+\boldsymbol{M} e^{-s \tau} \tilde{\boldsymbol{P}}(s) \boldsymbol{M}^{-1}\right) \tilde{\boldsymbol{Y}}_{c}(s)
\end{aligned}
$$


then a MoC macromodel, with $\tilde{\boldsymbol{Y}}(s)$ being defined in (2.13) as its admittance matrix, is passive if and only if both $\left[\boldsymbol{Y}_{1}(s)+\boldsymbol{Y}_{1}^{T}\left(s^{\star}\right)\right]$ and $\left[\boldsymbol{Y}_{2}(s)+\boldsymbol{Y}_{2}^{T}\left(s^{\star}\right)\right]$ are non-negative definite for $s=\jmath \omega, \omega \in \mathbb{R}$.

Proof Define the matrix $\mathfrak{Y}(\jmath \omega)$ as follows,

$$
\mathfrak{Y}(\jmath \omega)=\left[\begin{array}{cc}
\boldsymbol{Y}_{1}(\jmath \omega)+\boldsymbol{Y}_{1}^{T}(-\jmath \omega) & \mathbf{0} \\
\mathbf{0} & \boldsymbol{Y}_{2}(\jmath \omega)+\boldsymbol{Y}_{2}^{T}(-\jmath \omega)
\end{array}\right]
$$

It can be seen that $\mathfrak{Y}(\jmath \omega)$ is related to $\left[\tilde{\boldsymbol{Y}}^{T}(-\jmath \omega)+\tilde{\boldsymbol{Y}}(\jmath \omega)\right]$ by the following similarity transformation,

$$
\mathfrak{Y}(\jmath \omega)=\boldsymbol{J}\left[\tilde{\boldsymbol{Y}}^{T}(-\jmath \omega)+\tilde{\boldsymbol{Y}}(\jmath \omega)\right] \boldsymbol{J}^{-1}
$$

where,

$$
\boldsymbol{J}=\left[\begin{array}{cc}
\boldsymbol{I}_{m} & \boldsymbol{I}_{m} \\
-\boldsymbol{I}_{m} & \boldsymbol{I}_{m}
\end{array}\right]
$$

Therefore, the set of eigenvalues of $\mathfrak{Y}(\jmath \omega), \lambda\{\mathfrak{Y}(\jmath \omega)\}$, is obtained from

$$
\begin{aligned}
\lambda\{\mathfrak{Y}(\jmath \omega)\} & =\lambda\left\{\tilde{\boldsymbol{Y}}(\jmath \omega)+\tilde{\boldsymbol{Y}}^{T}(-\jmath \omega)\right\} \\
& =\lambda\left\{\boldsymbol{Y}_{1}(\jmath \omega)+\boldsymbol{Y}_{1}^{T}(-\jmath \omega)\right\} \cup \lambda\left\{\boldsymbol{Y}_{2}(\jmath \omega)+\boldsymbol{Y}_{2}^{T}(-\jmath \omega)\right\}
\end{aligned}
$$

From Lemma 4.2.7, it then follows that the MoC macromodel is passive if and only if $\operatorname{both}\left[\boldsymbol{Y}_{1}(s)+\boldsymbol{Y}_{1}^{T}\left(s^{\star}\right)\right]$ and $\left[\boldsymbol{Y}_{2}(s)+\boldsymbol{Y}_{2}^{T}\left(s^{\star}\right)\right]$ are non-negative definite for $s=\jmath \omega, \omega \in \mathbb{R}$.

The real advantage offered by the result of Lemma 4.3.8 stems from the fact that the task of verifying the passivity in MoC macromodels can be broken down to verifying the positive realness of the two artificial submodels with admittance matrices being defined in (4.2) and (4.3) on the $\jmath \omega$-axis. Although this might seem an unwieldy task, the structures of these admittance matrices enable deriving the necessary and sufficient conditions for the 
positive-realness without having to check all values of $\omega$. This issue will be illustrated in the following subsections.

\subsubsection{Realization of $\boldsymbol{Y}_{1}(s)$ and $\boldsymbol{Y}_{2}(s)$}

This subsection presents the realization of $\boldsymbol{Y}_{1}(s)$ and $\boldsymbol{Y}_{2}(s)$ in the time-domain in the form of dynamical differential equations with delay elements. A systematic way to obtain this representation is given by the following two propositions.

Proposition 4.3.2 $Y_{1}(s)$, defined in (4.2), can be represented by two dynamical systems with delays, defined as $\Sigma_{1}$ and $\Sigma_{2}$, respectively, and one linear time invariant (LTI) system, defined as $\Sigma_{3}$, and described as follows,

$$
\begin{aligned}
& \Sigma_{1}:\left\{\begin{aligned}
\mathcal{K}_{1} \dot{\boldsymbol{\chi}}(t) & =\mathcal{A}_{1} \boldsymbol{\chi}(t)+\mathcal{B}_{1} \boldsymbol{u}_{1}(t) \\
\boldsymbol{y}_{1}(t) & =\sum_{k=1}^{m} \mathcal{C}_{1}^{k} \boldsymbol{\chi}\left(t-\tau_{k}\right)+\mathcal{D}_{1} \boldsymbol{u}_{1}(t)
\end{aligned}\right. \\
& \Sigma_{2}:\left\{\begin{aligned}
\mathcal{K}_{2} \dot{\boldsymbol{\psi}}(t) & =\mathcal{A}_{2} \boldsymbol{\psi}(t)+\mathcal{B}_{2} \boldsymbol{u}_{2}(t) \\
\boldsymbol{u}_{1}(t) & =\sum \sum^{m} \mathcal{C}_{2}^{k} \boldsymbol{\psi}\left(t-\tau_{k}\right)+\mathcal{D}_{2} \boldsymbol{D}_{2} \boldsymbol{u}_{2}(t)
\end{aligned}\right. \\
& \Sigma_{3}:\left\{\begin{aligned}
\mathcal{K}_{3} \dot{\boldsymbol{Z}}(t) & =\mathcal{A}_{3} \boldsymbol{Z}(t)+\mathcal{B}_{3} \boldsymbol{u}_{3}(t) \\
\boldsymbol{u}_{2}(t) & =\mathcal{C}_{3} \boldsymbol{Z}(t)+\mathcal{D}_{3} \boldsymbol{u}_{3}(t)
\end{aligned}\right.
\end{aligned}
$$

with $\tau_{k}$ being the $k^{\text {th }}$ diagonal element of the matrix $\tau$. The matrices describing $\Sigma_{1}, \Sigma_{2}$ and $\Sigma_{3}$ are given in terms of the coefficients of the rational functions $\left(\tilde{\boldsymbol{P}}(s)\right.$ and $\left.\tilde{\boldsymbol{Y}}_{c}(s)\right)$, as shown in Appendix $C$.

Furthermore, let $\Pi_{1}(s), \Pi_{2}(s)$ and $\Pi_{3}(s)$ be the Laplace-domain transfer matrices for $\Sigma_{1}, \Sigma_{2}$ and $\Sigma_{3}$, respectively, then

$$
\boldsymbol{Y}_{1}(s)=\boldsymbol{\Pi}_{1}^{-1}(s) \boldsymbol{\Pi}_{2}(s) \boldsymbol{\Pi}_{3}(s)
$$

Proposition 4.3.3 $Y_{2}(s)$, defined in (4.3), can be represented by two dynamical systems 
with delays and one linear time invariant system, defined as $\hat{\Sigma}_{1}, \hat{\Sigma}_{2}$ and $\hat{\Sigma}_{3}$, respectively, and described as follows:

$$
\begin{gathered}
\hat{\Sigma}_{1}:\left\{\begin{aligned}
\mathcal{K}_{1} \dot{\boldsymbol{\chi}}(t) & =\mathcal{A}_{1} \boldsymbol{\chi}(t)+\mathcal{B}_{1} \hat{\boldsymbol{u}}_{1}(t) \\
\hat{\boldsymbol{y}}_{1}(t) & =-\sum_{k=1}^{m} \mathcal{C}_{1}^{k} \boldsymbol{\chi}\left(t-\tau_{k}\right)+\mathcal{D}_{1} \hat{\boldsymbol{u}}_{1}(t)
\end{aligned}\right. \\
\hat{\Sigma}_{2}:\left\{\begin{aligned}
\mathcal{K}_{2} \dot{\boldsymbol{\psi}}(t) & =\mathcal{A}_{2} \boldsymbol{\psi}(t)+\mathcal{B}_{2} \hat{\boldsymbol{u}}_{2}(t) \\
\hat{\boldsymbol{u}}_{1}(t) & =-\sum_{k=1}^{m} \mathcal{C}_{2}^{k} \boldsymbol{\psi}\left(t-\tau_{k}\right)+\mathcal{D}_{2} \hat{\boldsymbol{u}}_{2}(t)
\end{aligned}\right. \\
\hat{\Sigma}_{3}:\left\{\begin{aligned}
\mathcal{K}_{3} \dot{\boldsymbol{Z}}(t) & =\mathcal{A}_{3} \boldsymbol{Z}(t)+\mathcal{B}_{3} \hat{\boldsymbol{u}}_{3}(t) \\
\hat{\boldsymbol{u}}_{2}(t) & =\mathcal{C}_{3} \boldsymbol{Z}(t)+\mathcal{D}_{3} \hat{\boldsymbol{u}}_{3}(t)
\end{aligned}\right.
\end{gathered}
$$

Furthermore, let $\hat{\Pi}_{1}(s), \hat{\Pi}_{2}(s)$ and $\hat{\Pi}_{3}(s)$ be the Laplace-domain transfer matrices for $\hat{\Sigma}_{1}, \hat{\Sigma}_{2}$ and $\hat{\Sigma}_{3}$, respectively, then

$$
\boldsymbol{Y}_{2}(s)=\hat{\boldsymbol{\Pi}}_{1}^{-1}(s) \hat{\boldsymbol{\Pi}}_{2}(s) \hat{\boldsymbol{\Pi}}_{3}(s)
$$

The proof for Propositions 4.3.2 and 4.3.3 is given in Appendix C. It should be emphasized that the time-domain realization for MoC macromodels of MTLs is significantly different from the one presented earlier in Chapter 3 in the case of two-conductor TL, where a single delay system was shown to be the equivalent time-domain model. In this situation, it is shown that the MoC of the MTL maybe viewed as two-decoupled submodels each of which is composed of a cascade-like connection of two delay systems and one LTI system.

\subsubsection{Non-negative definiteness of $\boldsymbol{Y}_{1}(s)$ and $\boldsymbol{Y}_{2}(s)$}

The following theorem provides the necessary and sufficient conditions for the matrices $\left[\boldsymbol{Y}_{1}(\jmath \omega)+\boldsymbol{Y}_{1}^{T}(-\jmath \omega)\right]$ and $\left[\boldsymbol{Y}_{2}(\jmath \omega)+\boldsymbol{Y}_{2}^{T}(-\jmath \omega)\right]$ to be non-negative definite for $\omega \in \mathbb{R}$ via making use of the submodels realization in Proposition 4.3.2 and 4.3.3.

Theorem 4.3.4 The matrix $\left[\boldsymbol{Y}_{1}(\jmath \omega)+\boldsymbol{Y}_{1}^{T}(-\jmath \omega)\right]$ is non-negative definite $\forall \omega \in \mathbb{R}$ if and 
only if there are no simple pure imaginary eigenvalues satisfying the following frequency dependent-generalized eigenvalue problem,

$$
s \boldsymbol{K}_{1} \boldsymbol{\xi}=\boldsymbol{H}_{1}(s) \boldsymbol{\xi}
$$

Similarly, the matrix $\left[\boldsymbol{Y}_{2}(\jmath \omega)+\boldsymbol{Y}_{2}^{T}(-\jmath \omega)\right]$ is non-negative definite if and only if there are no simple pure imaginary eigenvalues satisfying the following FD-GEP,

$$
s \boldsymbol{K}_{2} \boldsymbol{\xi}=\boldsymbol{H}_{2}(s) \boldsymbol{\xi}
$$

The matrices $\boldsymbol{K}_{1}, \boldsymbol{K}_{2}, \boldsymbol{H}_{1}(s)$ and $\boldsymbol{H}_{2}(s)$ are given in terms of the matrices defining the systems $\Sigma_{1}, \Sigma_{2}, \Sigma_{3}, \hat{\Sigma}_{1}, \hat{\Sigma}_{2}$ and $\hat{\Sigma}_{3}$, and their structures will be laid out in proving the theorem.

Proof The following paragraphs give the proof of the first part of the theorem statement which is related to $\left[\boldsymbol{Y}_{1}(\jmath \omega)+\boldsymbol{Y}_{1}^{T}(-\jmath \omega)\right]$. The proof of the second part can be derived in $a$ similar manner.

Denote the set of eigenvalues of $\left[\boldsymbol{Y}_{1}(\jmath \omega)+\boldsymbol{Y}_{1}^{T}(-\jmath \omega)\right]$ by $\left\{\lambda_{k}(\jmath \omega)\right\}$. Assume that for each $k$, there is at least one value of $\omega$, denoted by $\omega_{k}$, for which $\lambda_{k}\left(\jmath \omega_{k}\right)>0$. It is therefore natural to conclude that the matrix $\left[\boldsymbol{Y}_{1}(\jmath \omega)+\boldsymbol{Y}_{1}^{T}(-\jmath \omega)\right]$ will cease to be nonnegative definite if there happened to be an interval on the $\jmath \omega$-axis for which $\lambda_{k}(\jmath \omega)<0$, or, alternatively, if there is a point, say $\omega_{o}$, at which $\lambda_{k}\left(\jmath \omega_{0}\right)=0$. To prove the necessity part of the theorem, we first assume that the matrix $\left[\boldsymbol{Y}_{1}(\jmath \omega)+\boldsymbol{Y}_{1}^{T}(-\jmath \omega)\right]$ is not non-negative definite at some interval on the $\jmath \omega$-axis, and hence there is a point $\omega_{0}$ at which $\lambda_{k}\left(\jmath \omega_{o}\right)=0$ and therefore the matrix $\left[\boldsymbol{Y}_{1}\left(\jmath \omega_{0}\right)+\boldsymbol{Y}_{1}^{T}\left(-\jmath \omega_{o}\right)\right]$ is singular. We now proceed to show that the point $s_{o}=\jmath \omega_{o}$ satisfies the eigenvalue problem of (4.10). Given that the matrix 
$\left[\boldsymbol{Y}_{1}\left(\jmath \omega_{0}\right)+\boldsymbol{Y}_{1}^{T}\left(-\jmath \omega_{0}\right)\right]$ is singular, then

$$
\left[\boldsymbol{\Pi}_{1}\left(s_{o}\right)^{-1} \boldsymbol{\Pi}_{2}\left(s_{o}\right) \boldsymbol{\Pi}_{3}\left(s_{o}\right)+\boldsymbol{\Pi}_{3}^{T}\left(s_{o}^{\star}\right) \boldsymbol{\Pi}_{2}^{T}\left(s_{o}^{\star}\right) \boldsymbol{\Pi}_{1}^{T}\left(s_{o}^{\star}\right)^{-1}\right] \boldsymbol{\rho}=\mathbf{0}
$$

or, alternatively, by pre-multiplying by $\Pi_{1}\left(s_{o}\right)$ and letting $\varphi=\Pi_{1}^{T}\left(s_{o}^{\star}\right)^{-1} \rho$,

$$
\left[\boldsymbol{\Pi}_{2}\left(s_{o}\right) \boldsymbol{\Pi}_{3}\left(s_{o}\right) \boldsymbol{\Pi}_{1}^{T}\left(s_{o}^{\star}\right)+\Pi_{1}\left(s_{o}\right) \boldsymbol{\Pi}_{3}^{T}\left(s_{o}^{\star}\right) \Pi_{2}^{T}\left(s_{o}^{\star}\right)\right] \boldsymbol{\varphi}=\mathbf{0}
$$

Substituting (C.12)-(C.14) into (4.13) and using several mathematical manipulations yield,

$$
\begin{aligned}
\varphi=\hat{\mathcal{R}}\left[\mathcal{C}_{2}\left(s_{o}\right) \delta+\mathcal{D}_{2} \mathcal{C}_{3} \boldsymbol{\gamma}\right. & +\mathcal{D}_{2} \mathcal{D}_{3} \mathcal{B}_{1}^{T} \boldsymbol{\theta} \\
& \left.+\mathcal{C}_{1}\left(s_{o}\right) \boldsymbol{\beta}+\mathcal{D}_{1} \mathcal{B}_{3}^{T} \boldsymbol{\mu}+\mathcal{D}_{1} \mathcal{D}_{3}^{T} \mathcal{B}_{2}^{T} \boldsymbol{\alpha}\right]
\end{aligned}
$$

where

$$
\begin{aligned}
\hat{\mathcal{R}} & =-\left(\mathcal{D}_{2} \mathcal{D}_{3} \mathcal{D}_{1}^{T}+\mathcal{D}_{1} \mathcal{D}_{3}^{T} \mathcal{D}_{2}^{T}\right)^{-1} \\
\boldsymbol{\beta} & =\left(s_{o} \mathcal{K}_{1}-\mathcal{A}_{1}\right)^{-1}\left(\mathcal{B}_{1} \mathcal{B}_{3}^{T} \boldsymbol{\mu}+\mathcal{B}_{1} \mathcal{D}_{3}^{T} \mathcal{B}_{2}^{T} \boldsymbol{\alpha}+\mathcal{B}_{1} \mathcal{D}_{3}^{T} \mathcal{D}_{2}^{T} \boldsymbol{\varphi}\right) \\
\boldsymbol{\theta} & =-\left(s_{o} \mathcal{K}_{1}^{T}+\mathcal{A}_{1}^{T}\right)^{-1} \mathcal{C}_{1}^{T}\left(s_{o}^{\star}\right) \boldsymbol{\varphi} \\
\boldsymbol{\delta} & =\left(s_{o} \mathcal{K}_{2}-\mathcal{A}_{2}\right)^{-1}\left(\mathcal{B}_{2} \mathcal{C}_{3} \gamma+\mathcal{B}_{2} \mathcal{D}_{3} \mathcal{B}_{1}^{T} \boldsymbol{\theta}+\mathcal{B}_{2} \mathcal{D}_{3} \mathcal{D}_{1}^{T} \boldsymbol{\varphi}\right) \\
\boldsymbol{\alpha} & =-\left(s_{o} \mathcal{K}_{2}^{T}+\mathcal{A}_{2}^{T}\right)^{-1} \mathcal{C}_{2}^{T}\left(s_{o}^{\star}\right) \boldsymbol{\varphi} \\
\boldsymbol{\gamma} & =\left(s_{o} \mathcal{K}_{3}-\mathcal{A}_{3}\right)^{-1}\left(\mathcal{B}_{3} \mathcal{B}_{1}^{T} \boldsymbol{\theta}+\mathcal{B}_{3} \mathcal{D}_{1}^{T} \boldsymbol{\varphi}\right) \\
\boldsymbol{\mu} & =-\left(s_{o} \mathcal{K}_{3}^{T}+\mathcal{A}_{3}^{T}\right)^{-1}\left(\mathcal{C}_{3}^{T} \mathcal{B}_{2}^{T} \boldsymbol{\alpha}+\mathcal{C}_{3}^{T} \mathcal{D}_{2}^{T} \boldsymbol{\varphi}\right)
\end{aligned}
$$

Substituting (4.14) into (4.16)-(4.21), respectively, using some manipulations and writing in a matrix form yield,

$$
s_{o} \boldsymbol{K}_{1} \boldsymbol{\vartheta}=\boldsymbol{H}_{1}\left(s_{o}\right) \boldsymbol{\vartheta}
$$


where

$$
\begin{aligned}
& \vartheta=\left[\boldsymbol{\beta}^{T} \boldsymbol{\delta}^{T} \boldsymbol{\gamma}^{T} \boldsymbol{\theta}^{T} \boldsymbol{\alpha}^{T} \boldsymbol{\mu}^{T}\right]^{T} \\
& \boldsymbol{K}_{1}=\operatorname{diag}\left\{\mathcal{K}_{1}, \mathcal{K}_{2}, \mathcal{K}_{3}, \mathcal{K}_{1}^{T}, \mathcal{K}_{2}^{T}, \mathcal{K}_{3}^{T},\right\} \\
& \boldsymbol{H}_{1}\left(s_{o}\right)=\left[\begin{array}{ll}
\boldsymbol{H}_{11}\left(s_{o}\right) & \boldsymbol{H}_{12}\left(s_{o}\right) \\
\boldsymbol{H}_{21}\left(s_{o}\right) & \boldsymbol{H}_{22}\left(s_{o}\right)
\end{array}\right] \\
& \boldsymbol{H}_{11}\left(s_{o}\right)=\left[\begin{array}{ccc}
\mathcal{A}_{1}+\hat{\mathcal{B}}_{1} \hat{\mathcal{R}} \mathcal{C}_{1}\left(s_{o}\right) & \hat{\mathcal{B}}_{1} \hat{\mathcal{R}} \mathcal{C}_{2}\left(s_{o}\right) & \hat{\mathcal{B}}_{1} \hat{\mathcal{R}} \hat{\mathcal{C}}_{3} \\
\hat{\mathcal{B}}_{2} \hat{\mathcal{R}} \mathcal{C}_{1}\left(s_{o}\right) & \mathcal{A}_{2}+\hat{\mathcal{B}}_{2} \hat{\mathcal{R}} \mathcal{C}_{2}\left(s_{o}\right) & \hat{\mathcal{B}}_{2} \hat{\mathcal{R}} \hat{\mathcal{C}}_{3}+\mathcal{B}_{2} \mathcal{C}_{3} \\
\hat{\mathcal{B}}_{3} \hat{\mathcal{R}} \mathcal{C}_{1}\left(s_{o}\right) & \hat{\mathcal{B}}_{3} \hat{\mathcal{R}} \mathcal{C}_{2}\left(s_{o}\right) & \mathcal{A}_{3}+\hat{\mathcal{B}}_{3} \hat{\mathcal{R}} \hat{\mathcal{C}}_{3}
\end{array}\right] \\
& \boldsymbol{H}_{12}\left(s_{o}\right)=\left[\begin{array}{ccc}
\hat{\mathcal{B}}_{1} \hat{\mathcal{R}} \hat{\mathcal{B}}_{1}^{T} & \hat{\mathcal{B}}_{1} \hat{\mathcal{R}} \hat{\mathcal{B}}_{2}^{T}+\mathcal{B}_{1} \mathcal{D}_{3}^{T} \mathcal{B}_{2}^{T} & \hat{\mathcal{B}}_{1} \hat{\mathcal{R}} \hat{\mathcal{B}}_{3}^{T}+\mathcal{B}_{1} \mathcal{B}_{3}^{T} \\
\hat{\mathcal{B}}_{2} \hat{\mathcal{R}} \hat{\mathcal{B}}_{1}^{T}+\mathcal{B}_{2} \mathcal{D}_{3} \mathcal{B}_{1}^{T} & \hat{\mathcal{B}}_{2} \hat{\mathcal{R}} \hat{\mathcal{B}}_{2}^{T} & \hat{\mathcal{B}}_{2} \hat{\mathcal{R}} \hat{\mathcal{B}}_{3}^{T} \\
\hat{\mathcal{B}}_{3} \hat{\mathcal{R}} \hat{\mathcal{B}}_{1}^{T}+\mathcal{B}_{3} \mathcal{B}_{1}^{T} & \hat{\mathcal{B}}_{3} \hat{\mathcal{R}} \hat{\mathcal{B}}_{2}^{T} & \hat{\mathcal{B}}_{3} \hat{\mathcal{R}}^{T} \hat{\mathcal{B}}_{3}^{T}
\end{array}\right] \\
& \boldsymbol{H}_{21}\left(s_{o}\right)=\left[\begin{array}{ccc}
-\mathcal{C}_{1}^{T}\left(s_{o}^{\star}\right) \hat{\mathcal{R}} \mathcal{C}_{1}\left(s_{o}\right) & -\mathcal{C}_{1}^{T}\left(s_{o}^{\star}\right) \hat{\mathcal{R}} \mathcal{C}_{2}\left(s_{o}\right) & -\mathcal{C}_{1}^{T}\left(s_{o}^{\star}\right) \hat{\mathcal{R}} \hat{\mathcal{C}}_{3} \\
-\mathcal{C}_{2}^{T}\left(s_{o}^{\star}\right) \hat{\mathcal{R}} \mathcal{C}_{1}\left(s_{o}\right) & -\mathcal{C}_{2}^{T}\left(s_{o}^{\star}\right) \hat{\mathcal{R}} \mathcal{C}_{2}\left(s_{o}\right) & -\mathcal{C}_{2}^{T}\left(s_{o}^{\star}\right) \hat{\mathcal{R}} \hat{\mathcal{C}}_{3} \\
-\hat{\mathcal{C}}_{3}^{T} \hat{\mathcal{R}} \mathcal{C}_{1}\left(s_{o}\right) & -\hat{\mathcal{C}}_{3}^{T} \hat{\mathcal{R}} \mathcal{C}_{2}\left(s_{o}\right) & -\hat{\mathcal{C}}_{3}^{T} \hat{\mathcal{R}} \hat{\mathcal{C}}_{3}
\end{array}\right] \\
& \boldsymbol{H}_{22}\left(s_{o}\right)=\left[\begin{array}{ccc}
-\mathcal{A}_{1}^{T}-\mathcal{C}_{1}^{T}\left(s_{o}^{\star}\right) \hat{\mathcal{R}} \hat{\mathcal{B}}_{1}^{T} & -\mathcal{C}_{1}^{T}\left(s_{o}^{\star}\right) \hat{\mathcal{R}} \hat{\mathcal{B}}_{2}^{T} & -\mathcal{C}_{1}^{T}\left(s_{o}^{\star}\right) \hat{\mathcal{R}} \hat{\mathcal{B}}_{3}^{T} \\
-\mathcal{C}_{2}^{T}\left(s_{o}^{\star}\right) \hat{\mathcal{R}} \hat{\mathcal{B}}_{1}^{T} & -\mathcal{A}_{2}^{T}-\mathcal{C}_{2}^{T}\left(s_{o}^{\star}\right) \hat{\mathcal{R}} \hat{\mathcal{B}}_{2}^{T} & -\mathcal{C}_{2}^{T}\left(s_{o}^{\star}\right) \hat{\mathcal{R}} \hat{\mathcal{B}}_{3}^{T} \\
-\hat{\mathcal{C}}_{3}^{T} \hat{\mathcal{R}} \hat{\mathcal{B}}_{1}^{T} & -\hat{\mathcal{C}}_{3}^{T} \hat{\mathcal{R}} \hat{\mathcal{B}}_{2}^{T}-\mathcal{C}_{3}^{T} \mathcal{B}_{2}^{T} & -\mathcal{A}_{3}^{T}-\hat{\mathcal{C}}_{3}^{T} \hat{\mathcal{R}} \hat{\mathcal{B}}_{3}^{T}
\end{array}\right] \\
& \hat{\mathcal{B}}_{1}=\mathcal{B}_{1} \mathcal{D}_{3}^{T} \mathcal{D}_{2}^{T}, \quad \hat{\mathcal{B}}_{3}=\mathcal{B}_{3} \mathcal{D}_{1}^{T} \\
& \hat{\mathcal{B}}_{2}=\mathcal{B}_{2} \mathcal{D}_{3} \mathcal{D}_{1}^{T}, \quad \hat{\mathcal{C}}_{3}=\mathcal{D}_{2} \mathcal{C}_{3}
\end{aligned}
$$

Hence if zero is a simple eigenvalue of $\left[\boldsymbol{Y}_{1}\left(s_{o}\right)+\boldsymbol{Y}_{1}^{T}\left(s_{o}^{\star}\right)\right]$, then $s_{o}$ must be a simple eigenvalue of $s \boldsymbol{K}_{1} \vartheta=\boldsymbol{H}_{1}(s) \vartheta$. This proves the necessity part.

Proving the sufficiency part can be achieved through working in a reverse manner by assuming that $s_{o}=j \omega_{o}$ satisfies

$$
s_{o} \boldsymbol{K}_{1} \boldsymbol{\vartheta}=\boldsymbol{H}_{1}\left(s_{o}\right) \boldsymbol{\vartheta}
$$


and then proceeding to show that $\left[\boldsymbol{Y}_{1}\left(s_{o}\right)+\boldsymbol{Y}_{1}^{T}\left(s_{o}^{\star}\right)\right]$ is singular. The detailed steps are omitted here for brevity.

The above theorem extends the Theorem 3.3.1 in Chapter 3, where a single ADDEs was considered, to handle the more general case including two delay systems and one LTI system given in Propositions 4.3.2 and 4.3.3.

\subsubsection{Regions of passivity violation}

While Lemma 4.3.8 and Theorem 4.3.4 specify the necessary and sufficient conditions for a given $\mathrm{MoC}$ macromodel to lose passivity, they stop short of locating those regions on the $\jmath \omega$-axis at which the eigenvalues $\lambda_{k}(s)$ of $\left[\tilde{\boldsymbol{Y}}(s)+\tilde{\boldsymbol{Y}}^{T}\left(s^{\star}\right)\right]$ become negative, and render the macromodel non-passive. The following theorem is aimed at addressing this issue.

Theorem 4.3.5 The passivity violation regions of a non-passive MoC macromodel are delimited by those points of $s$ on the jw-axis that are the simple eigenvalues of (4.10) and (4.11).

Proof Proof of Theorem 4.3.5 follows easily from Lemma 4.3.8 and Theorem 4.3.4, in that if $s_{o}\left(=\jmath \omega_{0}\right)$ is a simple imaginary eigenvalue of (4.10) and (4.11), then at least one of the eigenvalues of $\left[\tilde{\boldsymbol{Y}}\left(s_{o}\right)+\tilde{\boldsymbol{Y}}^{T}\left(s_{o}^{\star}\right)\right]$ becomes zero. In other words, at least one of the eigenvalues of $\left[\tilde{\boldsymbol{Y}}(\jmath \omega)+\tilde{\boldsymbol{Y}}^{T}(-\jmath \omega)\right]$ will have its value changing from positive to negative or vice versa as $\omega \rightarrow \omega_{o}$ from both sides. Therefore, the regions of passivity violation will always be delimited by those imaginary points satisfying (4.10) and (4.11).

The above theorem is a generalization of Theorem 3.3.2 in Chapter 3 for the case of a two-conductor TL. Based on Lemma 4.3.8 and Theorems 4.3.4 and 4.3.5, we can draw the following conclusions. If it is possible to compute the set of all solutions of $s$ that satisfy (4.10) and (4.11), and this set does not contain any simple purely imaginary eigenvalues, 
then one can conclude that (4.1) is satisfied and therefore the macromodel is a guaranteed passive macromodel. If, on the other hand, there happens to be one or more simple purely imaginary solutions for $s$ that satisfy (4.10) or (4.11), then the MoC macromodel is nonpassive. Furthermore, the regions of passivity violation are delimited by those imaginary solutions. The important issue that remains to be addressed is related to solving the FDGEPs in (4.10) and (4.11) developed in Theorem 4.3.4 for simple imaginary eigenvalues. This is presented in the following section.

\subsection{Computational considerations}

The problem of finding imaginary values for $s$ satisfying (4.10) and (4.11) can be cast as a problem of finding real values of $\omega$ satisfying the following eigenvalue problems,

$$
\begin{aligned}
\jmath \omega \boldsymbol{K}_{1} \boldsymbol{\xi} & =\boldsymbol{H}_{1}(\jmath \omega) \boldsymbol{\xi} \\
\jmath \omega \boldsymbol{K}_{2} \boldsymbol{\xi} & =\boldsymbol{H}_{2}(\jmath \omega) \boldsymbol{\xi}
\end{aligned}
$$

This type of problems cannot be treated as a classical eigenvalue problem since the associated characteristic polynomial is of the transcendental type [93,94]. Several approaches based on Newton-Raphson method have appeared in the literature to handle this type of problems [95]. Other techniques based on homotopy type methods have also been proposed to find the transcendental eigenvalues [96]. The approach employed here, however, is based on adapting an algorithm introduced earlier to handle the special case arising from macromodels of only two conductors [30]. This approach is based on noting that $\boldsymbol{H}_{1}(s)$ and $\boldsymbol{H}_{2}(s)$ can be written in the following form:

$$
\boldsymbol{H}_{1}(s)=\mathcal{V}^{(1)}+\sum_{k=1}^{m} \hat{\boldsymbol{W}}_{k}^{(1)} e^{-s \tau_{k}}+\sum_{k=1}^{m} \overline{\boldsymbol{W}}_{k}^{(1)} e^{s \tau_{k}}+\sum_{k, l=1}^{m} \boldsymbol{W}_{k, l}^{(1)} e^{s\left(\tau_{k}-\tau_{l}\right)}
$$




$$
\boldsymbol{H}_{2}(s)=\mathcal{V}^{(2)}+\sum_{k=1}^{m} \hat{\boldsymbol{W}}_{k}^{(2)} e^{-s \tau_{k}}+\sum_{k=1}^{m} \overline{\boldsymbol{W}}_{k}^{(2)} e^{s \tau_{k}}+\sum_{k, l=1}^{m} \boldsymbol{W}_{k, l}^{(2)} e^{s\left(\tau_{k}-\tau_{l}\right)}
$$

where $\mathcal{V}^{(1)}, \hat{\boldsymbol{W}}_{k}^{(1)}, \overline{\boldsymbol{W}}_{k}^{(1)}$ and $\boldsymbol{W}_{k, l}^{(1)}$ are $6 \times 6$ block $s$-independent matrices given by,

$$
\begin{aligned}
& \mathcal{V}^{(1)}=\left[\begin{array}{cccc}
\mathcal{A}_{1} & \mathbf{0} & \hat{\mathcal{B}}_{1} \hat{\mathcal{R}} \hat{\mathcal{C}}_{3} & \hat{\mathcal{B}}_{1} \hat{\mathcal{R}} \hat{\mathcal{B}}_{1}^{T} \\
\mathbf{0} & \mathcal{A}_{2} & \hat{\mathcal{B}}_{2} \hat{\mathcal{R}} \hat{\mathcal{C}}_{3}+\mathcal{B}_{2} \mathcal{C}_{3} & \hat{\mathcal{B}}_{2} \hat{\mathcal{R}} \hat{\mathcal{B}}_{1}^{T}+\mathcal{B}_{2} \mathcal{D}_{3} \mathcal{B}_{1}^{T} \\
\mathbf{0} & \mathbf{0} & \mathcal{A}_{3}+\hat{\mathcal{B}}_{3} \hat{\mathcal{R}} \hat{\mathcal{C}}_{3} & \hat{\mathcal{B}}_{3} \hat{\mathcal{R}} \hat{\mathcal{B}}_{1}^{T}+\mathcal{B}_{3} \mathcal{B}_{1}^{T} \\
\mathbf{0} & \mathbf{0} & \mathbf{0} & -\mathcal{A}_{1}^{T} \\
\mathbf{0} & 0 & 0 & 0 \\
\mathbf{0} & 0 & -\hat{\mathcal{C}}_{3}^{T} \hat{\mathcal{R}} \hat{\mathcal{C}}_{3} & -\hat{\mathcal{C}}_{3}^{T} \hat{\mathcal{R}} \hat{\mathcal{B}}_{1}^{T}
\end{array}\right. \\
& \hat{\mathcal{B}}_{1} \hat{\mathcal{R}} \hat{\mathcal{B}}_{2}^{T}+\mathcal{B}_{1} \mathcal{D}_{3}^{T} \mathcal{B}_{2}^{T} \quad \hat{\mathcal{B}}_{1} \hat{\mathcal{R}} \hat{\mathcal{B}}_{3}^{T}+\mathcal{B}_{1} \mathcal{B}_{3}^{T} \\
& \hat{\mathcal{B}}_{2} \hat{\mathcal{R}} \hat{\mathcal{B}}_{2}^{T} \quad \hat{\mathcal{B}}_{2} \hat{\mathcal{R}} \hat{\boldsymbol{B}}_{3}^{T}
\end{aligned}
$$

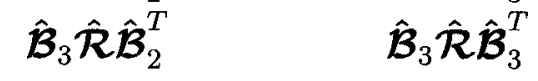

$$
\begin{aligned}
& 0 \quad 0 \\
& -\mathcal{A}_{2}^{T} \quad 0 \\
& \left.-\hat{\mathcal{C}}_{3}^{T} \hat{\mathcal{R}} \hat{\mathcal{B}}_{2}^{T}-\mathcal{C}_{3}^{T} \mathcal{B}_{2}^{T} \quad-\mathcal{A}_{3}^{T}-\hat{\mathcal{C}}_{3}^{T} \hat{\mathcal{R}} \hat{\mathcal{B}}_{3}^{T}\right] \\
& \hat{\boldsymbol{W}}_{k}^{(1)}=\left[\begin{array}{cccccc}
\hat{\mathcal{B}}_{1} \hat{\mathcal{R}} \mathcal{C}_{1}^{k} & \hat{\mathcal{B}}_{1} \hat{\mathcal{R}} \mathcal{C}_{2}^{k} & \mathbf{0} & \mathbf{0} & \mathbf{0} & 0 \\
\hat{\mathcal{B}}_{2} \hat{\mathcal{R}} \mathcal{C}_{1}^{k} & \hat{\mathcal{B}}_{2} \hat{\mathcal{R}} \mathcal{C}_{2}^{k} & \mathbf{0} & \mathbf{0} & \mathbf{0} & 0 \\
\hat{\mathcal{B}}_{3} \hat{\mathcal{R}} \mathcal{C}_{1}^{k} & \hat{\mathcal{B}}_{3} \hat{\mathcal{R}} \mathcal{C}_{2}^{k} & \mathbf{0} & \mathbf{0} & \mathbf{0} & \mathbf{0} \\
\mathbf{0} & \mathbf{0} & \mathbf{0} & \mathbf{0} & \mathbf{0} & \mathbf{0} \\
\mathbf{0} & \mathbf{0} & \mathbf{0} & \mathbf{0} & \mathbf{0} & \mathbf{0} \\
-\hat{\mathcal{C}}_{3}^{T} \hat{\mathcal{R}} \mathcal{C}_{1}^{k} & -\hat{\mathcal{C}}_{3}^{T} \hat{\mathcal{R}} \mathcal{C}_{2}^{k} & 0 & \mathbf{0} & 0 & \mathbf{0}
\end{array}\right] \\
& \overline{\boldsymbol{W}}_{k}^{(1)}=\left[\begin{array}{cccccc}
\mathbf{0} & \mathbf{0} & \mathbf{0} & \mathbf{0} & \mathbf{0} & \mathbf{0} \\
\mathbf{0} & \mathbf{0} & \mathbf{0} & \mathbf{0} & \mathbf{0} & \mathbf{0} \\
\mathbf{0} & \mathbf{0} & \mathbf{0} & \mathbf{0} & \mathbf{0} & \mathbf{0} \\
\mathbf{0} & \mathbf{0} & -\left[\mathcal{C}_{1}^{k}\right]^{T} \hat{\mathcal{R}} \hat{\mathcal{C}}_{3} & -\left[\mathcal{C}_{1}^{k}\right]^{T} \hat{\mathcal{R}} \hat{\mathcal{B}}_{1}^{T} & -\left[\mathcal{C}_{1}^{k}\right]^{T} \hat{\mathcal{R}} \hat{\mathcal{B}}_{2}^{T} & -\left[\mathcal{C}_{1}^{k}\right]^{T} \hat{\mathcal{R}} \hat{\mathcal{B}}_{3}^{T} \\
\mathbf{0} & \mathbf{0} & -\left[\mathcal{C}_{2}^{k}\right]^{T} \hat{\mathcal{R}} \hat{\mathcal{C}}_{3} & -\left[\mathcal{C}_{2}^{k}\right]^{T} \hat{\mathcal{R}} \hat{\mathcal{B}}_{1}^{T} & -\left[\mathcal{C}_{2}^{k}\right]^{T} \hat{\mathcal{R}} \hat{\mathcal{B}}_{2}^{T} & -\left[\mathcal{C}_{2}^{k}\right]^{T} \hat{\mathcal{R}} \hat{\mathcal{B}}_{3}^{T} \\
\mathbf{0} & \mathbf{0} & \mathbf{0} & \mathbf{0} & \mathbf{0} & \mathbf{0}
\end{array}\right]
\end{aligned}
$$




$$
\boldsymbol{W}_{k, l}^{(1)}=\left[\begin{array}{cccccc}
\mathbf{0} & \mathbf{0} & \mathbf{0} & \mathbf{0} & \mathbf{0} & \mathbf{0} \\
\mathbf{0} & \mathbf{0} & \mathbf{0} & \mathbf{0} & \mathbf{0} & \mathbf{0} \\
\mathbf{0} & \mathbf{0} & \mathbf{0} & \mathbf{0} & \mathbf{0} & \mathbf{0} \\
-\left[\mathcal{C}_{1}^{k}\right]^{T} \hat{\mathcal{R}} \mathcal{C}_{1}^{l} & -\left[\mathcal{C}_{1}^{k}\right]^{T} \hat{\mathcal{R}} \mathcal{C}_{2}^{l} & \mathbf{0} & \mathbf{0} & \mathbf{0} & \mathbf{0} \\
-\left[\mathcal{C}_{2}^{k}\right]^{T} \hat{\mathcal{R}} \mathcal{C}_{1}^{l} & -\left[\mathcal{C}_{2}^{k}\right]^{T} \hat{\mathcal{R}} \mathcal{C}_{2}^{l} & \mathbf{0} & \mathbf{0} & \mathbf{0} & \mathbf{0} \\
\mathbf{0} & \mathbf{0} & \mathbf{0} & \mathbf{0} & \mathbf{0} & \mathbf{0}
\end{array}\right]
$$

$\mathcal{V}^{(2)}, \hat{W}_{k}^{(2)}, \bar{W}_{k}^{(2)}$ and $\boldsymbol{W}_{k, l}^{(2)}$ take the similar structure as $\mathcal{V}^{(1)}, \hat{W}_{k}^{(1)}, \bar{W}_{k}^{(1)}$ and $\boldsymbol{W}_{k, l}^{(1)}$, respectively, except that $\mathcal{C}_{1}^{k}$ and $\mathcal{C}_{2}^{k}$ are replaced by $-\mathcal{C}_{1}^{k}$ and $-\mathcal{C}_{2}^{k}$, respectively.

Note that $\boldsymbol{H}_{1}(s)$ and $\boldsymbol{H}_{2}(s)$ are composed from summations of constant matrices that are independent of $s$, whereas the summation coefficients are scalar functions of $s$ and given by the exponentials $e^{ \pm s \tau_{k}}$ and $e^{s\left(\tau_{k}-\tau_{l}\right)}, k, l=1,2, \ldots, m$. Therefore, restricting $s$ to purely imaginary values, i.e., $s=\jmath \omega$, will make $\boldsymbol{H}_{1}(s)$ and $\boldsymbol{H}_{2}(s)$ quasi-periodic in $\omega$, since the summation coefficients are periodic in $\omega$ with different periods. It follows that the generalized eigenvalues of the matrix pencils $\left(\boldsymbol{H}_{1}(\jmath \omega), \boldsymbol{K}_{1}\right)$ and $\left(\boldsymbol{H}_{2}(\jmath \omega), \boldsymbol{K}_{2}\right)$ are also quasi-periodic functions of $\omega$. Denoting those generalized eigenvalues by $\left\{\sigma_{k}(\jmath \omega)\right\}$, it can be deduced from Theorems 4.3.4 and 4.3.5 that passivity violation occurs at values of $\omega$ for which $\Re\left\{\sigma_{k}(\jmath \omega)\right\}=0$ while $\Im\left\{\sigma_{k}(\jmath \omega)\right\}=\omega$. Consequently, it may be stated that regions on the $\jmath \omega$-axis with passivity violation are likely to occur when $\left|\Re\left\{\sigma_{k}(\jmath \omega)\right\}\right|<\epsilon$ for small positive constant $\epsilon$. Given that $\Re\left\{\sigma_{k}(\jmath \omega)\right\}$ is quasi-periodic w.r.t. $\omega$ with a period of $\frac{2 \pi}{\tau_{o}}$, one can detect the potential passivity violation by investigating the values that $\sigma_{k}(\jmath \omega)$ takes in a single period, e.g. $0 \leq \omega<\frac{2 \pi}{\tau_{o}}$.

Nonetheless, the common period $\tau_{o}$ for general quasi-periodic waveforms may be very large, especially in the situation where the delays become arbitrarily close. The problem of dealing with the common period in general quasi-periodic signals has been addressed extensively in the context of steady-state analysis in non-linear circuits through the idea of artificial frequency mapping (AFM) [97-99]. The basic goal in this context is to sample 
the quasi-periodic signal at a rate that is at least twice the highest frequency for a period of time equal to the common period. However, in narrow band radio-frequency (RF) circuits, where excitation tones are typically close and fall in the $\mathrm{GHz}$ range, the number of sampling points become prohibitively large. Handling this problem through the AFM is approached by mapping the excitation tones (and their combinations) to a set of harmonically-related artificial frequency, thereby transforming the quasi-periodic problem into a periodic one with a well-defined, albeit artificial, period.

Although, in principal AFM can be used to address the present situation by letting the delays $\tau_{i}$ play the role of the excitation tones, and map those delays to an artificial delay, this may not be necessary since the basic interest in the current problem is the interval of potential passivity violation which is naturally limited by several factors independent from the actual common period $\tau_{o}$. At the forefront of these factors is the fact that the magnitude of $\sigma_{k}(\jmath \omega)$ is always a function of the elements in $\mathcal{V}^{(i)}, \hat{\boldsymbol{W}}_{k}^{(i)}, \overline{\boldsymbol{W}}_{k}^{(i)}$ and $\boldsymbol{W}_{k, l}^{(i)}$ (where $i=1,2$ ), and not $\omega$. This is easily seen from (4.28) and (4.29) where the $\omega$ variable only influences the phase of these elements. In other words, it is not possible to have an eigenvalue $\sigma_{k}(\jmath \omega)$ for which $\Im\left\{\sigma_{k}(\jmath \omega)\right\}=\omega$ for arbitrarily large $\omega$.

In addition, the finite precision with which the delays are typically specified entails that they can be represented by rational numbers, thus allowing a common delay to be found easily. In fact, experimental results show that retaining few significant decimal digit in storing the delay in nano-second does not affect the accuracy of the model in any noticeable way. This fact contributes further to shortening the search interval for potential passivity violations.

To obtain more accurate values for the passivity violation regions, we follow the procedure described in $[30,32]$, where each exponential function, $e^{j \omega \tau_{k}}$, is approximated with a 
suitable Chebyshev expansion. This results in a polynomial eigenvalue problem whose solutions yield the boundary of passivity violation regions accurately. A detailed description of this procedure is presented in Appendix D.

\subsection{Numerical examples}

The proposed approach was implemented in MATLAB ${ }^{\circledR}$ to check the passivity of three macromodels, generated through $\mathrm{MoC}$, for three transmission line networks. The results of these test cases are described in the following subsections. In the presentation, $\left\{\lambda_{k}(\jmath \omega)\right\}$ denotes the set of the eigenvalues of the matrix $\left[\tilde{\boldsymbol{Y}}(\jmath \omega)+\tilde{\boldsymbol{Y}}^{T}(-\jmath \omega)\right]$.

\subsubsection{Example 1}

This example is intended to show that the proposed approach in this chapter can also be applied to the special case of two-conductor TLs described in Chapter 3. For this purpose, the two-conductor TL in the first example of Chapter 3 was reconsidered here. The construction of the MoC-model is same as in Chapter 3. However, the difference presented here is that the generalized theory proposed in this chapter was applied to verify the passivity of the MoC-model.

Based on Propositions 4.3.2 and 4.3.3, the dynamical system representations for $\boldsymbol{Y}_{1}(s)$ and $\boldsymbol{Y}_{2}(s)$ were constructed and their associated FD-GEP were then considered to verify the passivity of the macromodel using the procedure described in Section 4.4. It was found that the FD-GEP corresponding to $Y_{1}(s)$ has four purely imaginary eigenvalues at,

$$
\jmath 1.9371 \times 10^{4}, \quad \jmath 1.5347 \times 10^{5}, \quad \jmath 3.9534 \times 10^{6}, \quad \jmath 3.7336 \times 10^{7}
$$

According to the theory developed in this chapter, the macromodel is non-passive where the passivity violation regions are delimited within the above frequency points. It is worth 
to note that these results are in a good agreement with the ones predicted in Chapter 3.

\subsubsection{Example 2}

In this example, a 3 -conductor $20 \mathrm{~cm}$ long TL with frequency-dependent PUL parameter matrices described in [85] was considered. First, a MoC macromodel was obtained by constructing rational approximations of orders $(6 / 6)$ and $(7 / 8)$ for $\boldsymbol{Y}_{c}(s)$ and $\boldsymbol{P}(s)$, respectively. The resulting macromodel was then considered for passivity verification using the proposed approach, where the FD-GEPs associated with submodels yielded 12 imaginary eigenvalues listed next.

$$
\begin{array}{llll}
\jmath 2.7013 \times 10^{8}, & \jmath 2.3036 \times 10^{8}, & \jmath 9.8286 \times 10^{7}, & \jmath 8.3084 \times 10^{7} \\
\jmath 2.0924 \times 10^{7}, & \jmath 1.7578 \times 10^{7}, & \jmath 4.1600 \times 10^{6}, & \jmath 3.6444 \times 10^{6} \\
\jmath 7.3861 \times 10^{5}, & \jmath 7.3210 \times 10^{5}, & \jmath 1.4813 \times 10^{5}, & \jmath 1.4224 \times 10^{5}
\end{array}
$$

According to the results of Theorem 4.3.5, this indicates that the MoC macromodel is non-passive with the eigenvalues of its admittance matrix $\left[\tilde{\boldsymbol{Y}}(\jmath \omega)+\tilde{\boldsymbol{Y}}^{T}(-\jmath \omega)\right]$ becoming negative in the intervals on the $\jmath \omega$-axis delimited by the above values.

To verify the above results, the four eigenvalues of $\left[\tilde{\boldsymbol{Y}}(\jmath \omega)+\tilde{\boldsymbol{Y}}^{T}(-\jmath \omega)\right]$ were computed for various values of $s$ on the $\jmath \omega$-axis. Figures 4.1 and 4.2 display all the eigenvalues of the matrix $\left[\tilde{\boldsymbol{Y}}(\jmath \omega)+\tilde{\boldsymbol{Y}}^{T}(-\jmath \omega)\right]$. It is clear from these figures that although two of those eigenvalues remain strictly positive for very high frequency (Figure 4.1), the other two eigenvalues become negative as seen in Figure 4.2. Closer inspection of those intervals at which these eigenvalues become negative on Figure 4.2 shows a good agreement with passivity violation points predicted using the proposed approach. 


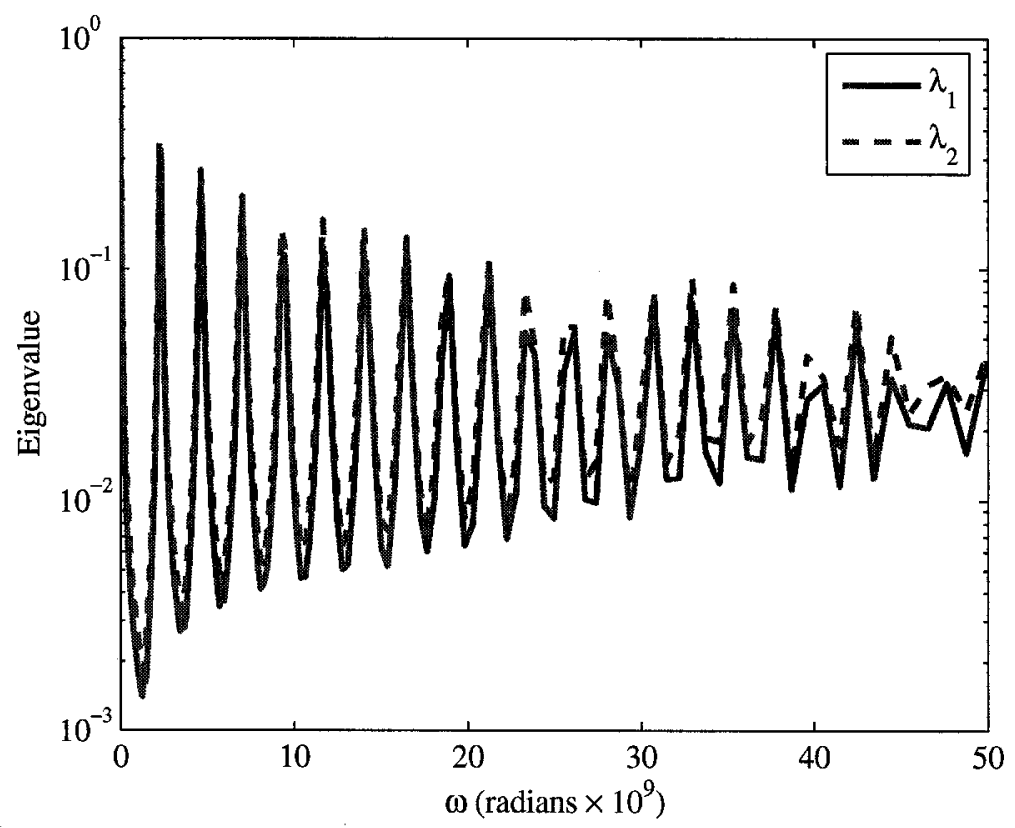

Figure 4.1: Eigenvalues of the first submodel.

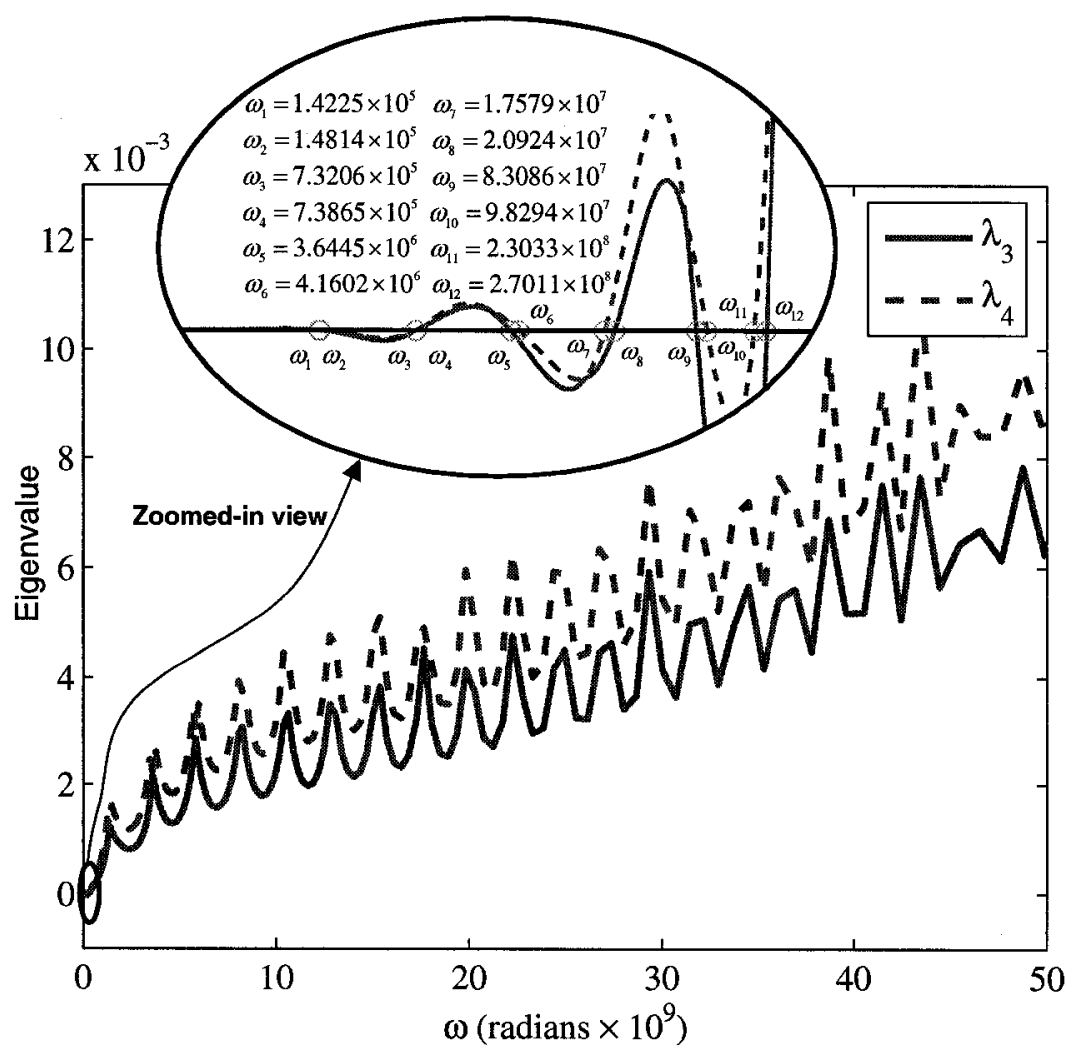

Figure 4.2: Eigenvalues of the second submodel. 


\subsubsection{Example 3}

In this example, a lossy frequency-dependent 11 -conductor TL with a length of $5 \mathrm{~cm}$ was considered for the proposed passivity verification approach. $\boldsymbol{Y}_{c}(s)$ and $\boldsymbol{P}(s)$ were approximated, in respective order, by $(5 / 5)$ and $(4 / 5)$ order matrix rational functions, which were used to construct the MoC-based macromodel as described previously. The passivity checking for the macromodel was completed by checking the non-negative definiteness of two sub-models based on Lemma 4.3.8. The two FD-GEPs associated with the two submodels were formulated and solved for imaginary eigenvalues. The simulation yielded no imaginary eigenvalues for the second sub-model, while 40 imaginary eigenvalues (listed in Table 4.1 under the column "Model") were found to satisfy the FD-GEP associated with the first submodel.

For the purpose of accuracy verification, Table 4.1 also lists those frequency points obtained via frequency sweeping. Each frequency point indicates that at least one of the eigenvalues of the macromodel admittance matrix becomes zero at that frequency point. A comparison between these values and those predicted earlier by the proposed theory shows a close agreement. Table 4.2 shows the CPU running time (in seconds) for each example on a Windows XP platform with a $1.5 G H z$ Intel Pentium IV processor. The CPU time for the "Frequency Sweeping" column is based on sweeping the frequency in the interval $\left[0,1 \times 10^{9}\right] \mathrm{Hz}$ with a step size of $0.1 \mathrm{MHz}$. It is noted that ideally the interval should be $[0, \infty] H z$ and the step size should be infinitely small. The CPU time based on the frequency sweeping approach is only provided here to demonstrate the computational efficiency of the proposed algorithm. 
Table 4.1: Numerical results for Example 3.

\begin{tabular}{|c|c||c|c||c|c|}
\hline Model & Frequency Sweeping & Model & Frequency Sweeping & Model & Frequency Sweeping \\
\hline \hline$\jmath 1.1788 \times 10^{8}$ & $\jmath 1.1786 \times 10^{8}$ & $\jmath 1.1266 \times 10^{8}$ & $\jmath 1.1266 \times 10^{8}$ & $\jmath 9.8421 \times 10^{7}$ & $\jmath 9.8405 \times 10^{7}$ \\
\hline$\jmath 1.5715 \times 10^{7}$ & $\jmath 1.5714 \times 10^{7}$ & $\jmath 1.9597 \times 10^{7}$ & $\jmath 1.9591 \times 10^{7}$ & $\jmath 1.9950 \times 10^{7}$ & $\jmath 1.9949 \times 10^{7}$ \\
\hline$\jmath 4.0434 \times 10^{6}$ & $\jmath 4.0448 \times 10^{6}$ & $\jmath 3.9846 \times 10^{6}$ & $\jmath 3.9871 \times 10^{6}$ & $\jmath 3.9535 \times 10^{6}$ & $\jmath 3.9544 \times 10^{6}$ \\
\hline$\jmath 1.0750 \times 10^{5}$ & $\jmath 1.0770 \times 10^{5}$ & $\jmath 1.1525 \times 10^{5}$ & $\jmath 1.1543 \times 10^{5}$ & $\jmath 1.2169 \times 10^{5}$ & $\jmath 1.2182 \times 10^{5}$ \\
\hline$\jmath 9.0912 \times 10^{7}$ & $\jmath 9.0891 \times 10^{7}$ & $\jmath 8.8970 \times 10^{7}$ & $\jmath 8.8956 \times 10^{7}$ & $\jmath 8.5749 \times 10^{7}$ & $\jmath 8.5736 \times 10^{7}$ \\
\hline$\jmath 2.0470 \times 10^{7}$ & $\jmath 2.0472 \times 10^{7}$ & $\jmath 2.1918 \times 10^{7}$ & $\jmath 2.1918 \times 10^{7}$ & $\jmath 2.3487 \times 10^{7}$ & $\jmath 2.3488 \times 10^{7}$ \\
\hline$\jmath 3.9441 \times 10^{6}$ & $\jmath 3.9443 \times 10^{6}$ & $\jmath 3.9333 \times 10^{6}$ & $\jmath 3.9343 \times 10^{6}$ & $\jmath 3.9237 \times 10^{6}$ & $\jmath 3.9239 \times 10^{6}$ \\
\hline$\jmath 1.2449 \times 10^{5}$ & $\jmath 1.2463 \times 10^{5}$ & $\jmath 1.2696 \times 10^{5}$ & $\jmath 1.2681 \times 10^{5}$ & $\jmath 1.3280 \times 10^{5}$ & $\jmath 1.3271 \times 10^{5}$ \\
\hline$\jmath 8.5348 \times 10^{7}$ & $\jmath 8.5329 \times 10^{7}$ & $\jmath 8.4098 \times 10^{7}$ & $\jmath 8.4094 \times 10^{7}$ & $\jmath 8.4084 \times 10^{7}$ & $\jmath 8.4060 \times 10^{7}$ \\
\hline$\jmath 2.3958 \times 10^{7}$ & $\jmath 2.3960 \times 10^{7}$ & $\jmath 2.5808 \times 10^{7}$ & $\jmath 2.5809 \times 10^{7}$ & $\jmath 2.9781 \times 10^{7}$ & $\jmath 2.9787 \times 10^{7}$ \\
\hline$\jmath 3.9119 \times 10^{6}$ & $\jmath 3.9162 \times 10^{6}$ & $\jmath 3.9117 \times 10^{6}$ & $\jmath 3.9080 \times 10^{6}$ & $\jmath 3.8606 \times 10^{6}$ & $\jmath 3.8583 \times 10^{6}$ \\
\hline$\jmath 1.3559 \times 10^{5}$ & $\jmath 1.3572 \times 10^{5}$ & $\jmath 1.3989 \times 10^{5}$ & $\jmath 1.3996 \times 10^{5}$ & $\jmath 1.4067 \times 10^{5}$ & $\jmath 1.4059 \times 10^{5}$ \\
\hline$\jmath 8.3750 \times 10^{7}$ & $\jmath 8.3701 \times 10^{7}$ & $\jmath 3.4988 \times 10^{7}$ & $\jmath 3.4991 \times 10^{7}$ & $\jmath 3.7905 \times 10^{6}$ & $\jmath 3.7887 \times 10^{6}$ \\
\hline$\jmath 1.5440 \times 10^{5}$ & $\jmath 1.5418 \times 10^{5}$ & & & & \\
\hline
\end{tabular}


Table 4.2: CPU time comparison (seconds).

\begin{tabular}{|l|c|c|}
\hline & Model & Frequency Sweeping \\
\hline \hline Example 1 & 0.26 & 29.7 \\
\hline Example 2 & 7.47 & 102.4 \\
\hline Example 3 & 181.67 & 2123.5 \\
\hline
\end{tabular}




\section{Chapter 5}

\section{Passivity Enforcement for Method of Characteristics Based Macromodels}

\subsection{Introduction}

In Chapters 3-4, a systematic procedure has been presented to verify the passivity in method of characteristics based macromodels for electrical high-speed interconnects. If the macromodel is non-passive, the passivity violation regions can be identified with a high accuracy. However, this is insuffficient in the practical applications, i.e., to include the MoCbased macromodels into non-linear circuit simulators for transient simulation, where passive macromodels are required. Verifying the passivity of the macromodel provides an important step in achieving a passive macromodel. Nevertheless, passivity enforcement is required in the next step to compensate the passivity violations for a non-passive macromodel. Several enforcement algorithms can be found in the literature $[59,64,66]$. However, in their current forms, they can only be applied to macromodels described in the form of ordinary differential equations. The passivity enforcement for MoC-based macromodels, which are in the forms of algebraic delay differential equations, still remains a challenging and open research topic. 
In order to address this issue, a new algorithm for the passivity enforcement of MoC based macromodels is proposed in this chapter [33]. The proposed algorithm iteratively compensates for passivity violating regions by perturbing the residues of the rational approximation of the characteristic admittance matrix [34] of the MTL. This perturbation is done under the constraint that the resulting error in the overall transfer function matrix of the MTL, is minimized. The development of the proposed algorithm is based on the formulation of MoC models for the general multi-conductor TLs.

The rest of the chapter is organized as follows. Section 5.2 presents the necessary theoretical foundations of the proposed passivity enforcement algorithm for MoC-based macromodels. Section 5.3 discusses the computational complexity of the proposed algorithm. Numerical examples are presented in Section 5.4.

\subsection{Proposed passivity enforcement algorithm}

The presentation of the proposed passivity enforcement algorithm assumes that the passivity violation regions have been identified using the algorithm described in Chapter 4 . This section presents the theoretical basis and the implementation procedure of the proposed algorithm. A two-step process is developed for this purpose. They are detailed in Sections 5.2.1 and 5.2.2.

\subsubsection{Determine the maximum passivity violation point in a PVR}

In this step, the maximum passivity violation location with its associated frequency point is identified for each passivity violation region. This location is identified by solving the following problem,

$$
\min _{\omega} \lambda(\Re\{\tilde{\boldsymbol{Y}}(\jmath \omega)\}) ; \quad \omega \in\left[\omega_{1}, \omega_{2}\right]
$$


where, $\omega_{1}$ and $\omega_{2}$ represent the lower and upper boundary frequency points that delimit the passivity violation region. $\lambda(\boldsymbol{X})$ denotes the function computing the eigenvalues of $\boldsymbol{X}$. Solving (5.1) provides us with a numerical quantity for each PVR, which has to be compensated for the macromodel to be passive.

Figure 5.1 illustrates the above idea graphically, where it can be seen that $\lambda_{\min }$ is the minimum eigenvalue of $\Re\{\tilde{\boldsymbol{Y}}(\jmath \omega)\}$ at $\omega=\omega_{\lambda}$, for a PVR delimited by frequency points $\omega_{1}$ and $\omega_{2}$. The problem in (5.1) converges very fast as it is associated with only one variable $(\omega)$ and has a good initial guess (midpoint) [59].

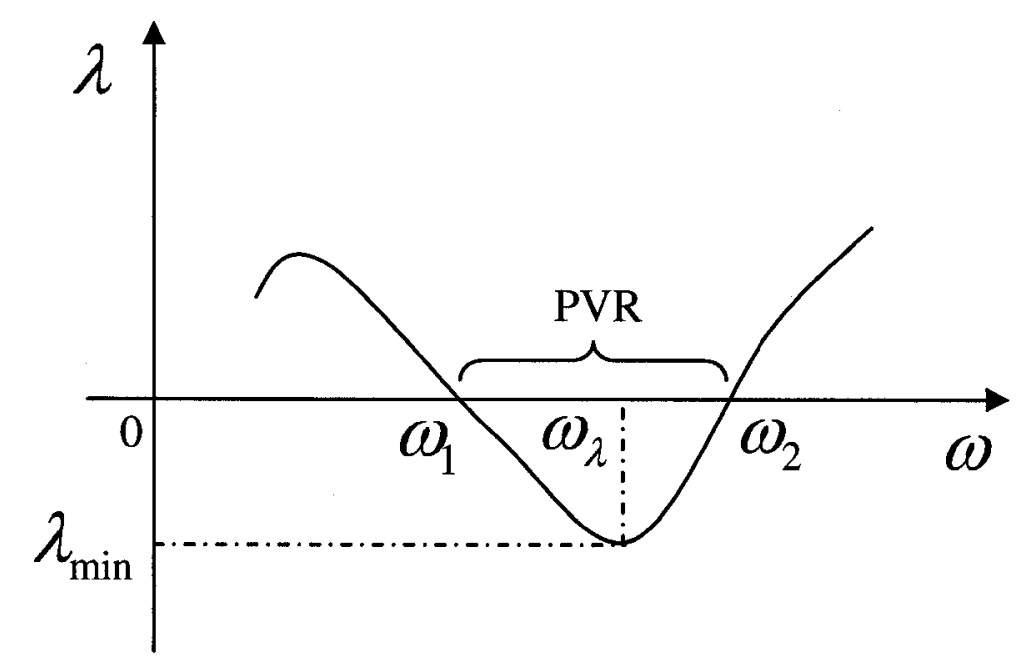

Figure 5.1: Illustration of the maximum passivity violation in a PVR.

\subsubsection{Proposed passivity enforcement}

This subsection describes the proposed passivity enforcement algorithm. It extends the scope of recently published passivity enforcement algorithms $[59,64,66]$ for macromodels based on ordinary differential equations to macromodels based on algebraic delayed differential equations obtained through MoC. The new method is designed to handle the general 
case of multi-conductor transmission lines. For this purpose, the passivity verification algorithm presented in Chapter 4 for general MTLs is utilized. The new algorithm is based on the first-order perturbation of the rational function approximation of $\tilde{\boldsymbol{Y}}_{c}(s)$, iteratively, until the macromodel becomes passive. The details of the procedure are as follows:

Let the rational approximation (C.6) of the characteristic admittance matrix $\tilde{\boldsymbol{Y}}_{c}(s)$ be represented by canonical state-space representation [100] as follows.

$$
\Sigma_{y c}:\left\{\begin{array}{l}
\dot{\boldsymbol{x}}_{y c}(t)=\boldsymbol{A}_{y c} \boldsymbol{x}_{y c}(t)+\boldsymbol{B}_{y c} \boldsymbol{u}_{y c}(t), \\
\boldsymbol{y}_{y c}(t)=\boldsymbol{C}_{y c} \boldsymbol{x}_{y c}(t)+\boldsymbol{D}_{y c} \boldsymbol{u}_{y c}(t),
\end{array}\right.
$$

where, $\boldsymbol{A}_{y c}$ is a block diagonal matrix including the poles of $\tilde{\boldsymbol{Y}}_{c}(s) ; \boldsymbol{B}_{y c}$ is the mapping matrix; $\boldsymbol{C}_{y c}$ includes the residues of $\tilde{\boldsymbol{Y}}_{c}(s)$ for each pole; and $\boldsymbol{D}_{y c}$ represents the direct coupling between the port voltages and currents of $\tilde{\boldsymbol{Y}}_{c}(s)$. It is evident from (5.2) that $\tilde{\boldsymbol{Y}}_{c}(s)$ can be expressed as

$$
\tilde{\boldsymbol{Y}}_{c}(s)=\boldsymbol{C}_{y c}\left(s \boldsymbol{I}-\boldsymbol{A}_{y c}\right)^{-1} \boldsymbol{B}_{y c}+\boldsymbol{D}_{y c}
$$

Next, substituting (5.3) into (2.13), replacing $s$ by $\jmath \omega$, and after some mathematical manipulations, the admittance matrix $\tilde{\boldsymbol{Y}}(\jmath \omega)$ of the macromodel can be expressed as

$$
\tilde{\boldsymbol{Y}}(\jmath \omega)=\boldsymbol{Y}_{R}(\jmath \omega)+\jmath \boldsymbol{Y}_{I}(\jmath \omega)
$$

where, $\boldsymbol{Y}_{R}(\jmath \omega)$ and $\boldsymbol{Y}_{I}(\jmath \omega)$ are the real and imaginary parts of $\tilde{\boldsymbol{Y}}(\jmath \omega)$, respectively, and defined as

$$
\begin{aligned}
& \boldsymbol{Y}_{R}(\jmath \omega)=\left[\begin{array}{ll}
\boldsymbol{Y}_{R 1} & \boldsymbol{Y}_{R 2} \\
\boldsymbol{Y}_{R 2} & \boldsymbol{Y}_{R 1}
\end{array}\right], \\
& \boldsymbol{Y}_{I}(\jmath \omega)=\left[\begin{array}{ll}
\boldsymbol{Y}_{I 1} & \boldsymbol{Y}_{I 2} \\
\boldsymbol{Y}_{I 2} & \boldsymbol{Y}_{I 1}
\end{array}\right],
\end{aligned}
$$


where,

$$
\begin{aligned}
\boldsymbol{Y}_{R 1} & =\hat{\boldsymbol{W}}_{1 R} C_{y} \boldsymbol{F}_{R}-\hat{\boldsymbol{W}}_{1 I} \boldsymbol{C}_{y} \boldsymbol{F}_{I}+\hat{\boldsymbol{W}}_{1 R} \boldsymbol{D}_{y} \\
\boldsymbol{Y}_{R 2} & =\hat{\boldsymbol{W}}_{2 R} \boldsymbol{C}_{y} \boldsymbol{F}_{R}-\hat{\boldsymbol{W}}_{2 I} \boldsymbol{C}_{y} \boldsymbol{F}_{I}+\hat{\boldsymbol{W}}_{2 R} \boldsymbol{D}_{y} \\
\boldsymbol{Y}_{I 1} & =\hat{\boldsymbol{W}}_{1 R} \boldsymbol{C}_{y} \boldsymbol{F}_{I}+\hat{\boldsymbol{W}}_{1 I} \boldsymbol{C}_{y} \boldsymbol{F}_{R}+\hat{\boldsymbol{W}}_{1 I} \boldsymbol{D}_{y} \\
\boldsymbol{Y}_{I 2} & =\hat{\boldsymbol{W}}_{2 R} \boldsymbol{C}_{y} \boldsymbol{F}_{I}+\hat{\boldsymbol{W}}_{2 I} \boldsymbol{C}_{y} \boldsymbol{F}_{R}+\hat{\boldsymbol{W}}_{2 I} \boldsymbol{D}_{y}
\end{aligned}
$$

Here, $\hat{\boldsymbol{W}}_{1 R}$ and $\hat{\boldsymbol{W}}_{1 I}$ represent the real and imaginary parts of $\boldsymbol{W}_{0}^{-1}(\jmath \omega) \boldsymbol{W}_{1}(\jmath \omega)$, respectively, $\hat{\boldsymbol{W}}_{2 R}$ and $\hat{\boldsymbol{W}}_{2 I}$ represent the real and imaginary parts of $\boldsymbol{W}_{0}^{-1}(\jmath \omega) \boldsymbol{W}_{2}(\jmath \omega)$, respectively, and $\boldsymbol{F}_{R}$ and $\boldsymbol{F}_{I}$ represent the real and imaginary parts of $\left(\jmath \omega \boldsymbol{I}-\boldsymbol{A}_{y c}\right)^{-1} \boldsymbol{B}_{y c}+\boldsymbol{D}_{y c}$, respectively. For the convenience of understanding, let the macromodel (5.4) be nonpassive and consisting of one passivity violation region. Further, let $\lambda_{\min }$ be the minimum value of the eigenvalues of $\boldsymbol{Y}_{R}(\jmath \omega)$ occurring at $\omega=\omega_{\lambda}$, as illustrated in Figure 5.1. In order to make the macromodel passive, we need to perturb certain parameters of the macromodel such that the minimum eigenvalue $\lambda_{\min }$ of $\boldsymbol{Y}_{R}\left(\jmath \omega_{\lambda}\right)$ is forced to become equal to or greater than zero. In other words, we seek a perturbation that will make the real part of the admittance matrix $\left(\boldsymbol{Y}_{R}(\jmath \omega)+\Delta \boldsymbol{Y}_{R}(\jmath \omega)\right)$ of the perturbed macromodel to be non-negative at $\omega=\omega_{\lambda}$. To achieve this objective, the residue matrix $\left(\boldsymbol{C}_{y c}\right)$ of the characteristic admittance matrix $\tilde{\boldsymbol{Y}}_{c}(s)$, is perturbed by an amount $\Delta \boldsymbol{C}_{y c}$. Denoting the updated residue matrix as $\hat{C}_{y c}=C_{y c}+\Delta C_{y c}$, the updated real part of the admittance matrix can be expressed as

$$
\boldsymbol{Y}_{R}(\jmath \omega)+\Delta \boldsymbol{Y}_{R}(\jmath \omega)=\left[\begin{array}{cc}
\hat{\boldsymbol{Y}}_{R 1} & \hat{\boldsymbol{Y}}_{R 2} \\
\hat{\boldsymbol{Y}}_{R 2} & \hat{\boldsymbol{Y}}_{R 1}
\end{array}\right]
$$

where,

$$
\begin{aligned}
& \hat{\boldsymbol{Y}}_{R 1}=\hat{\boldsymbol{W}}_{1 R} \hat{\boldsymbol{C}}_{y c} \boldsymbol{F}_{R}-\hat{\boldsymbol{W}}_{1 I} \hat{\boldsymbol{C}}_{y c} \boldsymbol{F}_{I}+\hat{\boldsymbol{W}}_{1 R} \boldsymbol{D}_{y c} \\
& \hat{\boldsymbol{Y}}_{R 2}=\hat{\boldsymbol{W}}_{2 R} \hat{\boldsymbol{C}}_{y c} \boldsymbol{F}_{R}-\hat{\boldsymbol{W}}_{2 I} \hat{\boldsymbol{C}}_{y c} \boldsymbol{F}_{I}+\hat{\boldsymbol{W}}_{2 R} \boldsymbol{D}_{y c} .
\end{aligned}
$$


It is evident that, the perturbation $\Delta \boldsymbol{Y}_{R}(\jmath \omega)$, in $\boldsymbol{Y}_{R}(\jmath \omega)$ can be expressed as

$$
\begin{array}{r}
\Delta \boldsymbol{Y}_{R}(\jmath \omega)=\left[\begin{array}{l}
\hat{\boldsymbol{W}}_{1 R} \Delta \hat{\boldsymbol{C}}_{y c} \boldsymbol{F}_{R}-\hat{\boldsymbol{W}}_{1 I} \Delta \hat{\boldsymbol{C}}_{y c} \boldsymbol{F}_{I} \\
\hat{\boldsymbol{W}}_{2 R} \Delta \hat{\boldsymbol{C}}_{y c} \boldsymbol{F}_{R}-\hat{\boldsymbol{W}}_{2 I} \Delta \hat{\boldsymbol{C}}_{y c} \boldsymbol{F}_{I} \\
\hat{\boldsymbol{W}}_{2 R} \Delta \hat{\boldsymbol{C}}_{y c} \boldsymbol{F}_{R}-\hat{\boldsymbol{W}}_{2 I} \Delta \hat{\boldsymbol{C}}_{y c} \boldsymbol{F}_{I} \\
\hat{\boldsymbol{W}}_{1 R} \Delta \hat{\boldsymbol{C}}_{y c} \boldsymbol{F}_{R}-\hat{\boldsymbol{W}}_{1 I} \Delta \hat{\boldsymbol{C}}_{y c} \boldsymbol{F}_{I}
\end{array}\right] .
\end{array}
$$

It is also evident that, a perturbation in $Y_{R}(\jmath \omega)$ will result in a perturbation of its eigenvalue too. If we represent the perturbation in an eigenvalue of $Y_{R}(\jmath \omega)$ with $\omega=\omega_{\lambda}$, by $\Delta \lambda$, then using the first-order perturbation theory [101], we can write

$$
\Delta \lambda \approx \frac{\boldsymbol{\vartheta} \Delta \boldsymbol{Y}_{R}\left(\jmath \omega_{\lambda}\right) \boldsymbol{\xi}}{\boldsymbol{\vartheta} \boldsymbol{\xi}}
$$

where, $\boldsymbol{\xi}$ and $\boldsymbol{\vartheta}$ are the right and left eigenvector of $\boldsymbol{Y}_{R}\left(\jmath \omega_{\lambda}\right)$, respectively, corresponding to the eigenvalue $\lambda_{\min }$. For the purpose of passivity compensation, we require that the eigenvalue of the perturbed matrix $\boldsymbol{Y}_{R}(\jmath \omega)+\Delta \boldsymbol{Y}_{R}(\jmath \omega)$ is non-negative at $\omega=\omega_{\lambda}$, i.e.,

$$
\lambda_{\min }+\Delta \lambda \geq 0
$$

Substituting (5.14)-(5.15) into (5.16), and after some mathematical manipulations, we can write

$$
\Theta_{w} \widehat{\Delta \boldsymbol{C}_{y c}} \Phi_{w} \geq-\lambda_{\min }
$$

where,

$$
\widehat{\Delta C_{y c}}=\left[\begin{array}{cccc}
\Delta C_{y c} & 0 & 0 & 0 \\
0 & \Delta C_{y c} & 0 & 0 \\
0 & 0 & \Delta C_{y c} & 0 \\
0 & 0 & 0 & \Delta C_{y c}
\end{array}\right]
$$


$\boldsymbol{\Theta}_{w}=\left.\boldsymbol{\vartheta}\left[\begin{array}{cccc}\hat{\boldsymbol{W}}_{1 R} & -\hat{\boldsymbol{W}}_{1 I} & \hat{\boldsymbol{W}}_{2 R} & -\hat{\boldsymbol{W}}_{2 I} \\ \hat{\boldsymbol{W}}_{2 R} & -\hat{\boldsymbol{W}}_{2 I} & \hat{\boldsymbol{W}}_{1 R} & -\hat{\boldsymbol{W}}_{1 I}\end{array}\right]\right|_{s=\jmath \omega_{\lambda}}$, and $\boldsymbol{\Phi}_{w}=\left.\left[\begin{array}{cc}\boldsymbol{F}_{R} & \mathbf{0} \\ \boldsymbol{F}_{I} & \mathbf{0} \\ \mathbf{0} & \boldsymbol{F}_{R} \\ \mathbf{0} & \boldsymbol{F}_{I}\end{array}\right] \boldsymbol{\xi}\right|_{s=\jmath \omega_{\lambda}}$

After certain mathematical manipulations, (5.17) can be written as

$$
\boldsymbol{\Psi}_{w} \boldsymbol{X}_{\Delta C} \geq-\lambda_{\min }
$$

where, matrix $\Psi_{w}$ is a function of $\vartheta, \hat{W}_{1 R}, \hat{W}_{1 I}, \hat{W}_{2 R}, \hat{W}_{2 I}, \omega_{\lambda}$ and $X_{\Delta C}$ is a column vector, consisting of the entries of unknown matrix $\Delta C_{y c}$, required for passivity enforcement. Solving (5.20) for the required perturbation $\Delta C_{y c}$ for passivity enforcement will result in a deviation of the original response of the system, denoted by $\Delta \tilde{\boldsymbol{Y}}(\jmath \omega)$. To minimize the error introduced in the original response of the system, we minimize the Frobenius norm of $\Delta \tilde{\boldsymbol{Y}}_{c}(\jmath \omega)$, which in turn minimizes the upper bound of the $L_{2}$-norm of the error introduced in the admittance matrix $\Delta \tilde{\boldsymbol{Y}}(j \omega)$. This can be understood by writing the $L_{2}$ norm of $\Delta \tilde{\boldsymbol{Y}}(\jmath \omega)$ as follows [59]

$$
\begin{aligned}
\|\Delta \tilde{\boldsymbol{Y}}\|_{2}^{2} & =\left\|\boldsymbol{\Xi}_{w}\left[\begin{array}{cc}
\Delta \tilde{\boldsymbol{Y}}_{c}(\jmath \omega) & \\
& \Delta \tilde{\boldsymbol{Y}}_{c}(\jmath \omega)
\end{array}\right]\right\|_{2}^{2} \\
& \leq\left\|\boldsymbol{\Xi}_{w}\right\|_{2}^{2}\left\|\left[\begin{array}{cc}
\Delta \tilde{\boldsymbol{Y}}_{c}(\jmath \omega) & \\
\Delta \tilde{\boldsymbol{Y}}_{c}(\jmath \omega)
\end{array}\right]\right\|_{2}^{2}, \\
& =2\left\|\boldsymbol{\Xi}_{w}\right\|_{2}^{2} \int_{-\infty}^{\infty}\left\|\Delta \tilde{\boldsymbol{Y}}_{c}(\jmath \omega)\right\|_{F}^{2} d \omega, \\
& =2\left\|\boldsymbol{\Xi}_{w}\right\|_{2}^{2} \int_{-\infty}^{\infty} \sum_{i=1}^{m} \sum_{j=1}^{m}\left|\Delta \tilde{\boldsymbol{Y}}_{c}^{(i, j)}(\jmath \omega)\right|^{2} d \omega, \\
& =2\left\|\boldsymbol{\Xi}_{w}\right\|_{2}^{2} \int_{-\infty}^{\infty} \sum_{i=1}^{m} \sum_{j=1}^{m}\left|\Delta \tilde{\boldsymbol{Y}}_{c}^{(i, j)}(t)\right|^{2} d t, \\
& =2\left\|\boldsymbol{\Xi}_{w}\right\|_{2}^{2} \operatorname{trace}\left(\Delta \boldsymbol{C}_{y c} \boldsymbol{\Omega}_{p} \Delta \boldsymbol{C}_{y c}^{T}\right),
\end{aligned}
$$

where,

$$
\boldsymbol{\Xi}_{w}=\left[\begin{array}{ll}
\boldsymbol{W}_{0}^{-1}(\jmath \omega) \boldsymbol{W}_{1}(\jmath \omega) & \boldsymbol{W}_{0}^{-1}(\jmath \omega) \boldsymbol{W}_{2}(\jmath \omega) \\
\boldsymbol{W}_{0}^{-1}(\jmath \omega) \boldsymbol{W}_{2}(\jmath \omega) & \boldsymbol{W}_{0}^{-1}(\jmath \omega) \boldsymbol{W}_{1}(\jmath \omega)
\end{array}\right]
$$


$\|\cdot\|_{2}$ and $\|\cdot\|_{F}$ denote the $L_{2}$ and Frobenius norms of the matrix, respectively, $m$ is the number of signal conductors in the transmission line and $\Omega_{p}$ is the controllability Grammian obtained by solving the following Lyapunov equation [102],

$$
\boldsymbol{A}_{y c} \boldsymbol{\Omega}_{p}+\boldsymbol{\Omega}_{p} \boldsymbol{A}_{y c}^{T}+\boldsymbol{B}_{y c} B_{y c}^{T}=0
$$

By leaving the matrices $\boldsymbol{A}_{y c}$ and $\boldsymbol{B}_{y c}$ unchanged and with matrix $\boldsymbol{\Omega}_{p}$ being constant, it is evident from (5.21) that by minimizing the trace of $\Delta C_{y c} \Omega_{p} \Delta C_{y c}^{T}$, the error in the time- and frequency-domain responses will be kept to a minimum value. In effect, the passivity compensation problem for a non-passive MoC macromodel is reduced to solving the following problem for $\Delta C_{y c}$ :

$$
\min _{\Delta \boldsymbol{C}_{y c}} \operatorname{trace}\left(\Delta \boldsymbol{C}_{y c} \boldsymbol{\Omega}_{p} \Delta \boldsymbol{C}_{y c}^{T}\right), \text { subject to (5.20). }
$$

It should be noted that, the above passivity enforcement algorithm is iterative in nature, with small perturbation applied to matrix $C_{y c}$ in each iteration. During the ensuing iterations, new passivity violations may be introduced, which are accounted for in the subsequent passivity enforcing iterations and the process converges quickly $[59,64,66]$. A brief summary of the proposed passivity enforcement algorithm is given in Figure 5.2 in the form of a psuedocode.

\subsection{Computational complexity}

This section provides a discussion on the computational complexity of the proposed passivity compensation algorithm for MoC-based macromodels. Each iteration involves passivity 


\section{Pseudocode of proposed passivity enforcement algorithm}

Step 1: Construct the MoC macromodel using rational functions $\tilde{P}(s)$ and $\tilde{Y}_{c}(s)$ as in (C.5)-(C.6).

Step 2: Construct the state-space representation for $\tilde{Y}_{c}(s)$ as in (5.2).

Step 3: Solve the Lyapunov equation (5.23) for $\boldsymbol{\Omega}_{p}$.

Step 4: Verify the passivity of the resulting MoC macromodel using the technique described in Chapter 4.

IF, there does not exist any passivity violation regions from the verification, the model is passive,

EXIT.

ELSE,

Step 5: Identify the passivity violating regions. Perform the passivity compensation, by solving (5.24) at the frequency points of maximum violation. Update the MoC macromodel by updating the residue matrix of $\tilde{Y}_{c}(s): \tilde{C}_{y c} \leftarrow C_{y c}+\Delta C_{y c}$.

Go to Step 4.

Figure 5.2: Pseudocode of the proposed passivity enforcement. 
verification of the MoC macromodel (2.13) (details in Chapter 4) and the passivity enforcement as outlined in Section 5.2.2. Passivity enforcement involves the quantification of passivity violation and solving for the enforcement problem in (5.24). The computational cost of this part is $O(n)$, where $n$ is the size of state-space system (5.2) of $\tilde{\boldsymbol{Y}}_{c}(s)$. Since, the characteristic admittance matrix is usually a relatively smooth function, the associated size of the state-space systems, $n$ is very small.

Solution of (5.23) for $\Omega_{p}$ is required to be performed only once and can be done with the efficient solution method described in [66], requiring a computational cost of $O(n)$.

\subsection{Numerical examples}

Several numerical examples are demonstrated in this section to validate the theory and the performance of the proposed algorithm.

\subsubsection{Example 1}

In this example, a 2-conductor transmission line of length $4 \mathrm{~cm}$ and PUL parameters: $R=$ $0.014 k \Omega / m, L=0.63 \mu H / m, G=0.001 \mathrm{mS} / \mathrm{m}, C=110 \mathrm{pf} / \mathrm{m}$ was considered. The MoC-based macromodel was generated by first approximating the characteristic admittance $\boldsymbol{Y}_{\boldsymbol{c}}(s)$ with 3 poles and the delayless operator $\boldsymbol{P}(s)$ with 2 poles, using vector fit [61]. Next, the resulting macromodel was verified for passivity using the procedure described in Chapter 4. The macromodel was found to be having four imaginary solutions for the FDGEP corresponding to $\boldsymbol{Y}_{1}(s)$ in (4.10), indicating that the model was non-passive (as per Theorem 4.3.4). Based on these four imaginary solutions, two passivity violation regions were identified as

$$
\left[\jmath 2.79 \times 10^{5}, \jmath 3.29 \times 10^{6}\right] \text { and }\left[\jmath 3.51 \times 10^{7}, \jmath 1.69 \times 10^{9}\right]
$$


and are indicated in Figure 5.3. Next, the maximum passivity violation points for each PVR was obtained and the proposed passivity enforcement problem (5.24) was solved to compute the required perturbation. The compensation process took 3 iterations to restore the passivity. Each iteration required 0.3 seconds for passivity verification and 0.3 seconds for passivity enforcement (Windows XP platform, $1.5 \mathrm{GHz}$ Pentium IV processor, MATLAB $\left.{ }^{\circledR}\right)$.

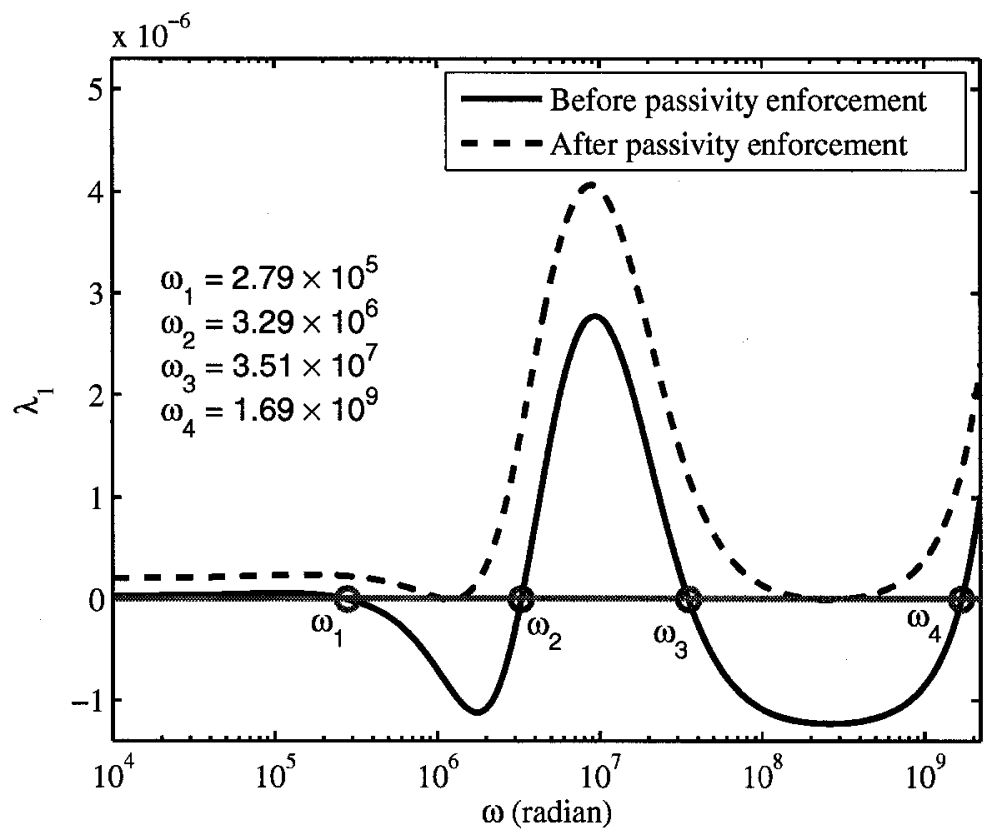

Figure 5.3: Eigenvalue spectrum of $\Re\{\tilde{\boldsymbol{Y}}(s)\}$ (Example 1).

It can be seen from Figure 5.3 that one of the eigenvalues for $\Re\{\tilde{\boldsymbol{Y}}(s)\}, \lambda_{1}$, which was negative before passivity enforcement, became non-negative throughout the frequency spectrum after the proposed passivity enforcement. It indicates that the macromodel became passive after enforcement. The passivity of the macromodel was also verified by solving frequency dependent generalized eigenvalue problem in (4.10) and (4.11)). Figure 5.4 and 5.5 show the comparison of $\left|Y_{11}\right|$ and $\left|Y_{12}\right|$, respectively, before and after passivity enforcement. As seen they match reasonably well. 


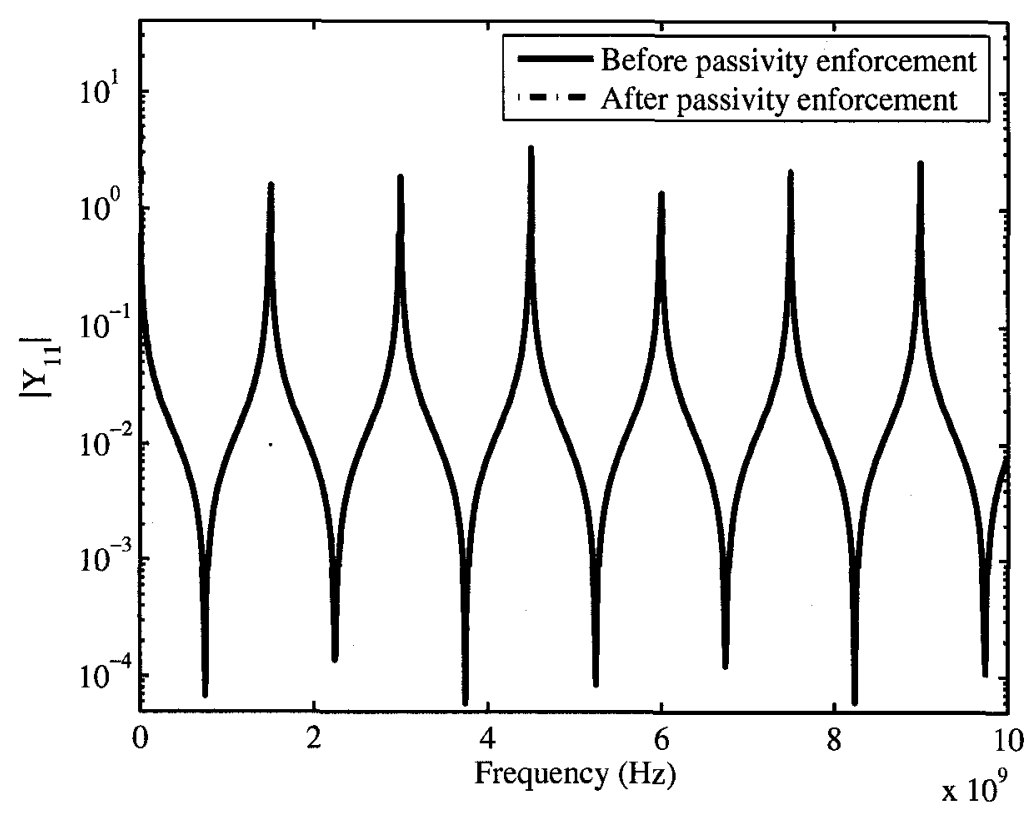

Figure 5.4: Comparison of frequency responses, $\left|\boldsymbol{Y}_{11}\right|$ (Example 1).

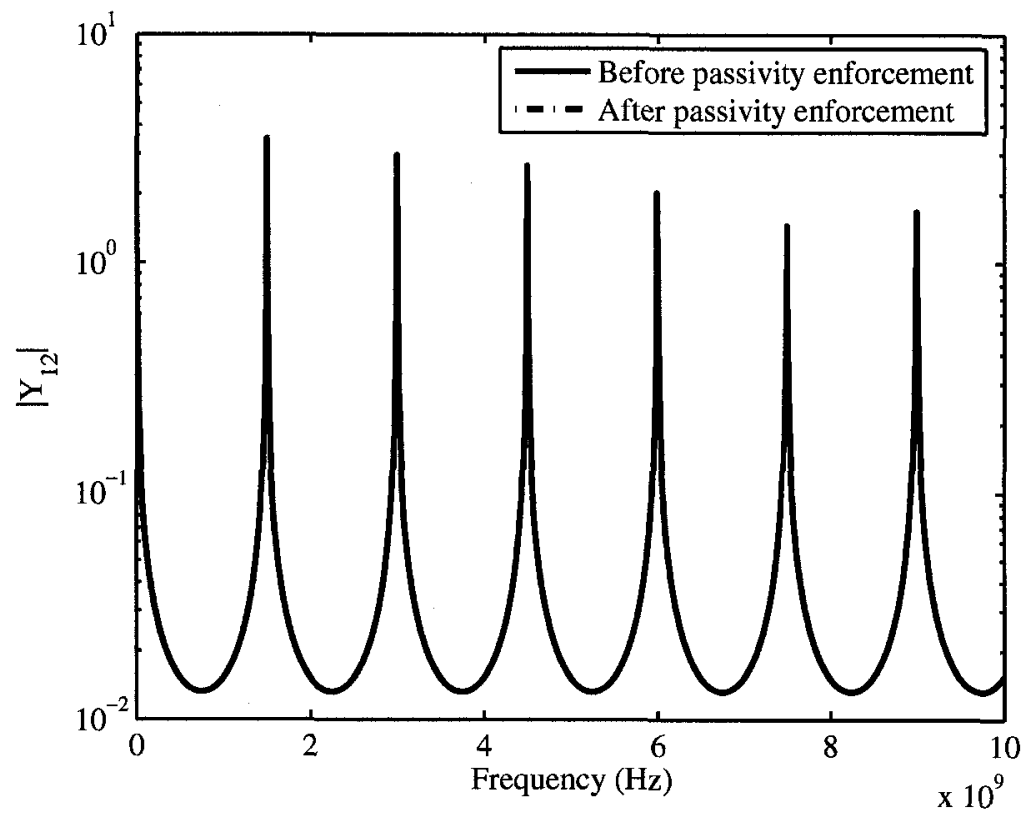

Figure 5.5: Comparison of frequency responses, $\left|\boldsymbol{Y}_{12}\right|$ (Example 1). 


\subsubsection{Example 2}

In this example, the same $\mathrm{MoC}$ macromodel as for Example 2 in Chapter 4 was considered. The macromodel was constructed for a 3-conductor $20 \mathrm{~cm}$ long transmission line with frequency-dependent PUL parameters. The orders of the rational approximation for $\boldsymbol{Y}_{c}(s)$ and $\boldsymbol{P}(s)$ during the modeling are 6 and 8 , respectively. Based on the approach described in Section 4.3, 12 imaginary eigenvalues were obtained for the associated FDGEPs. Three passivity violation regions were identified from these imaginary eigenvalues, as depicted in Figure 5.6, using the approach described in Chapter 4.

Next, this macromodel was compensated for passivity using the proposed passivity enforcement algorithm in Section 5.2.2. Three iterations were required for complete passivity enforcement. In each iteration, 7.5 seconds were required for passivity verification, while 0.6 seconds were required for passivity enforcement. To show that the compensation was successful, the eigenvalues of $\Re\{\tilde{\boldsymbol{Y}}(s)\}$ of the compensated macromodel are plotted in Figure 5.6. As is evident, the eigenvalues are non-negative throughout the frequency spectrum after passivity enforcement, and hence the macromodel is passive (passivity of the macromodel was also verified by solving frequency dependent-generalized eigenvalue problem in (4.10) and (4.11)). Figures 5.7, 5.8 and 5.9 display the comparison between the Y-parameters of the original non-passive model and the model after passivity enforcement and they match reasonably accurately. 


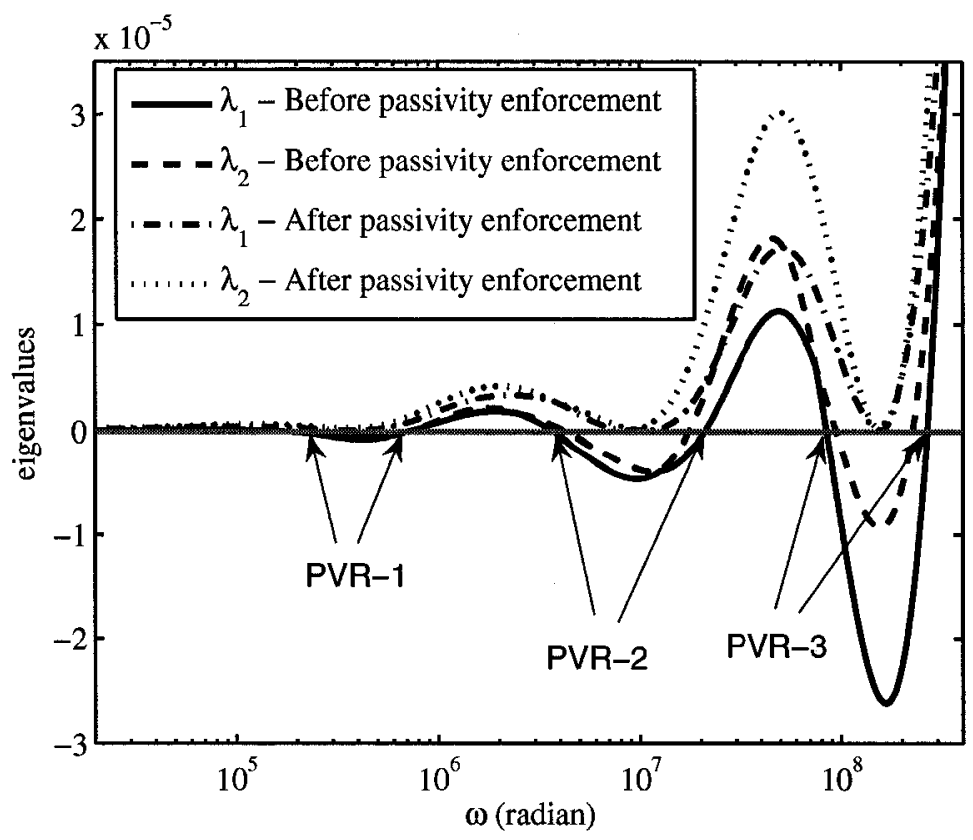

Figure 5.6: Eigenvalue spectrum of $\Re\{\tilde{\boldsymbol{Y}}(s)\}$ (Example 2).

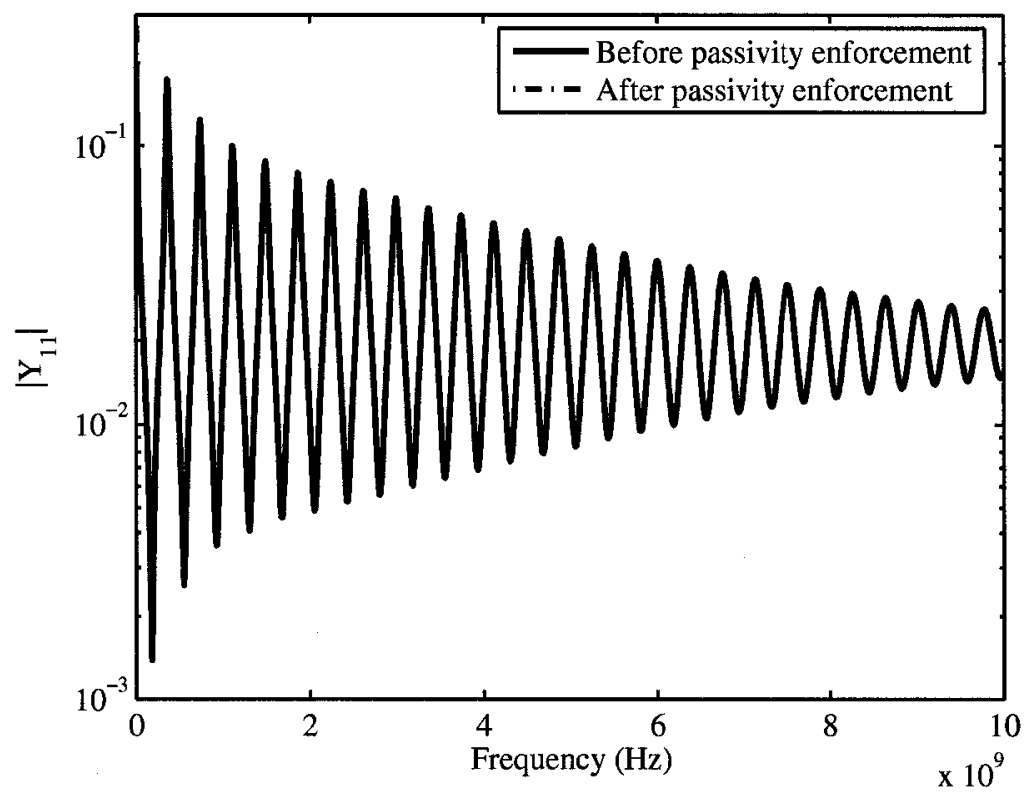

Figure 5.7: Comparison of frequency responses, $\left|\boldsymbol{Y}_{11}\right|$ (Example 2). 


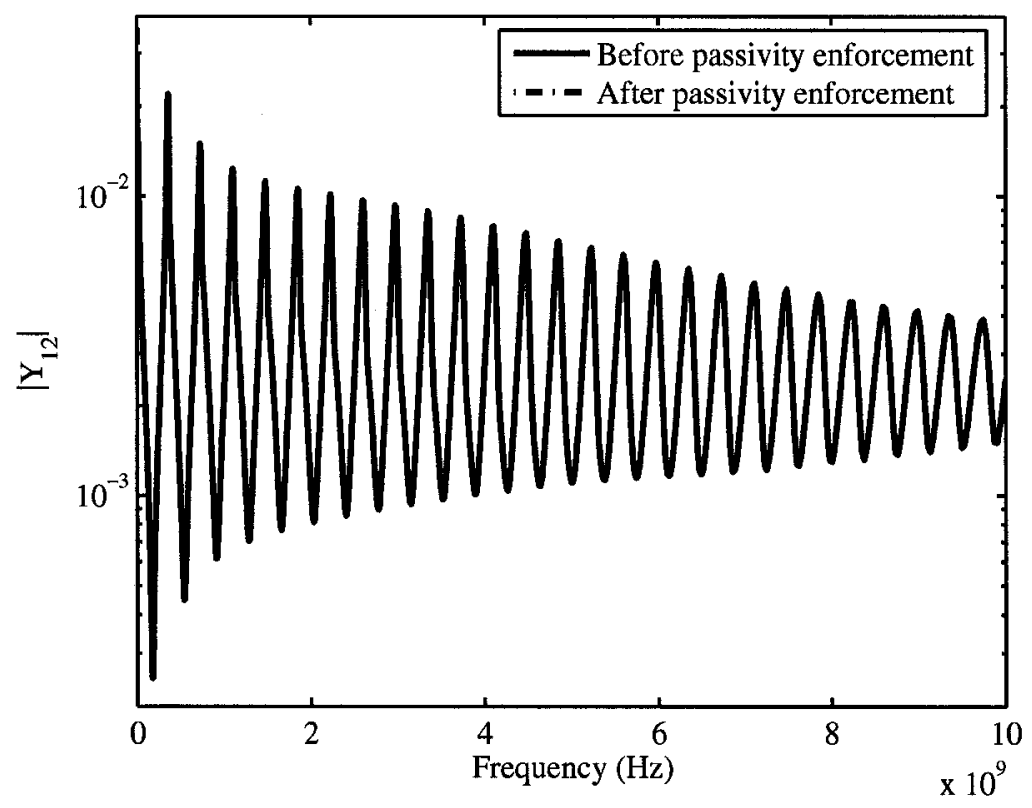

Figure 5.8: Comparison of frequency responses, $\left|\boldsymbol{Y}_{12}\right|$ (Example 2).

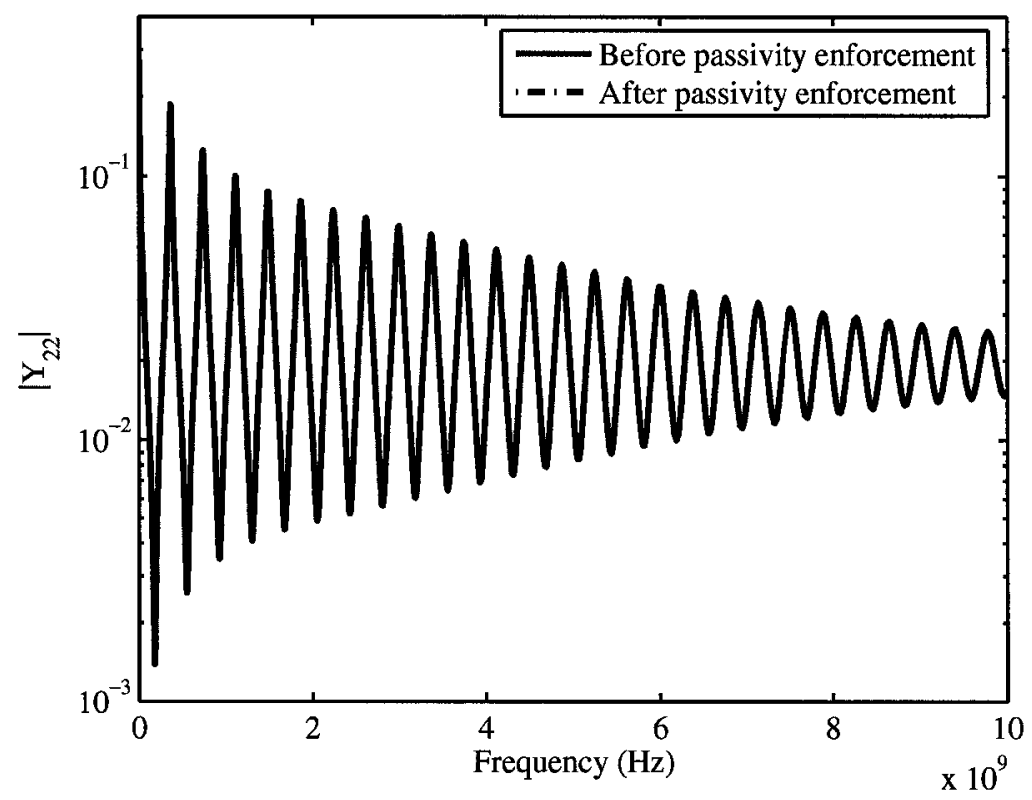

Figure 5.9: Comparison of frequency responses, $\left|\boldsymbol{Y}_{22}\right|$ (Example 2). 


\subsubsection{Example 3}

In this example, a 3 -conductor $12.5 \mathrm{~cm}$ long transmission line with the following PUL parameters was considered.

$$
\begin{aligned}
& \boldsymbol{R}=\left[\begin{array}{ccc}
0.0554 & 0 & 0 \\
0 & 0.0554 & 0 \\
0 & 0 & 0.0554
\end{array}\right] k \Omega / m \\
& \boldsymbol{L}=\left[\begin{array}{ccc}
0.2617 & 0.0488 & 0.0095 \\
0.0488 & 0.2555 & 0.0488 \\
0.0095 & 0.0488 & 0.2617
\end{array}\right] \mu H / m \\
& \boldsymbol{G}=\left[\begin{array}{ccc}
40.7140 & -7.7750 & -0.0226 \\
-7.7750 & 43.1930 & -7.7750 \\
-0.0226 & -7.7750 & 40.7140
\end{array}\right] * 10^{-3} \mathrm{mS} / \mathrm{m}, \\
& \boldsymbol{C}=\left[\begin{array}{ccc}
185.1400 & -35.3550 & -0.1026 \\
-35.3550 & 196.4100 & -35.3550 \\
-0.1026 & -35.3550 & 185.1400
\end{array}\right] p f / m .
\end{aligned}
$$

First, a MoC macromodel was obtained by constructing rational approximations of order 5 for $\boldsymbol{Y}_{c}(s)$ and $\boldsymbol{P}(s)$. The resulting macromodel was then verified for passivity using the approach described in Section 4.3, where the solution of FD-GEPs resulted in 4 imaginary eigenvalues, implying that the macromodel was not passive. Based on these four imaginary solutions, one passivity violation region was identified as

$$
\left[\jmath 4.26 \times 10^{7}, \jmath 6.78 \times 10^{7}\right]
$$

Next, this macromodel was compensated for passivity using the proposed passivity enforcement algorithm in Section 5.2.2. A total of two iterations were required for complete 
passivity enforcement. In each iteration 9.31 seconds were required for passivity verification, while 0.4 seconds were required for passivity enforcement. To show that the compensation was successful, the eigenvalues of $\Re\{\tilde{\boldsymbol{Y}}(s)\}$ of the compensated macromodel are plotted in Figure 5.10 and 5.11. As is evident, the eigenvalues are non-negative throughout the frequency spectrum after passivity enforcement, and hence the macromodel is passive (passivity of the macromodel was also verified by solving frequency dependent-generalized eigenvalue problem in (4.10) and (4.11)). Figures 5.12, 5.13 and 5.14 display the comparison between the sample Y-parameters of the original non-passive model and the model after passivity enforcement and they match reasonably well.

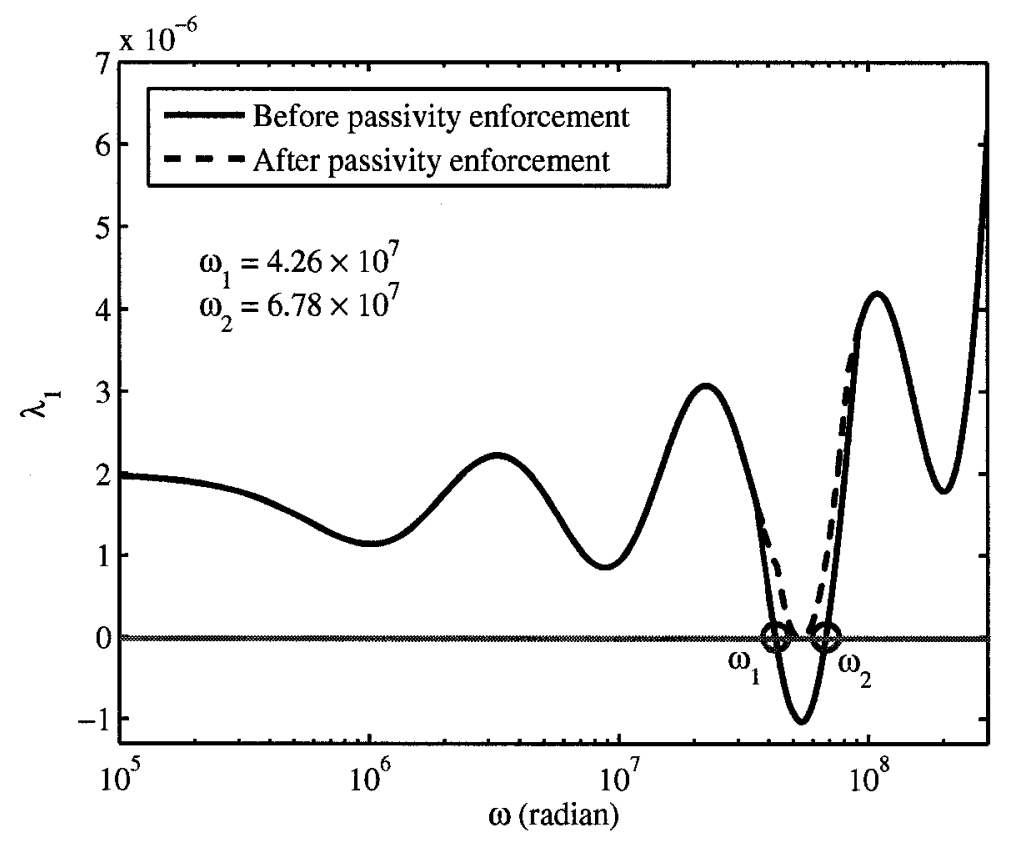

Figure 5.10: Eigenvalue spectrum of $\Re\{\tilde{\boldsymbol{Y}}(s)\}, \lambda_{1}$ (Example 3). 


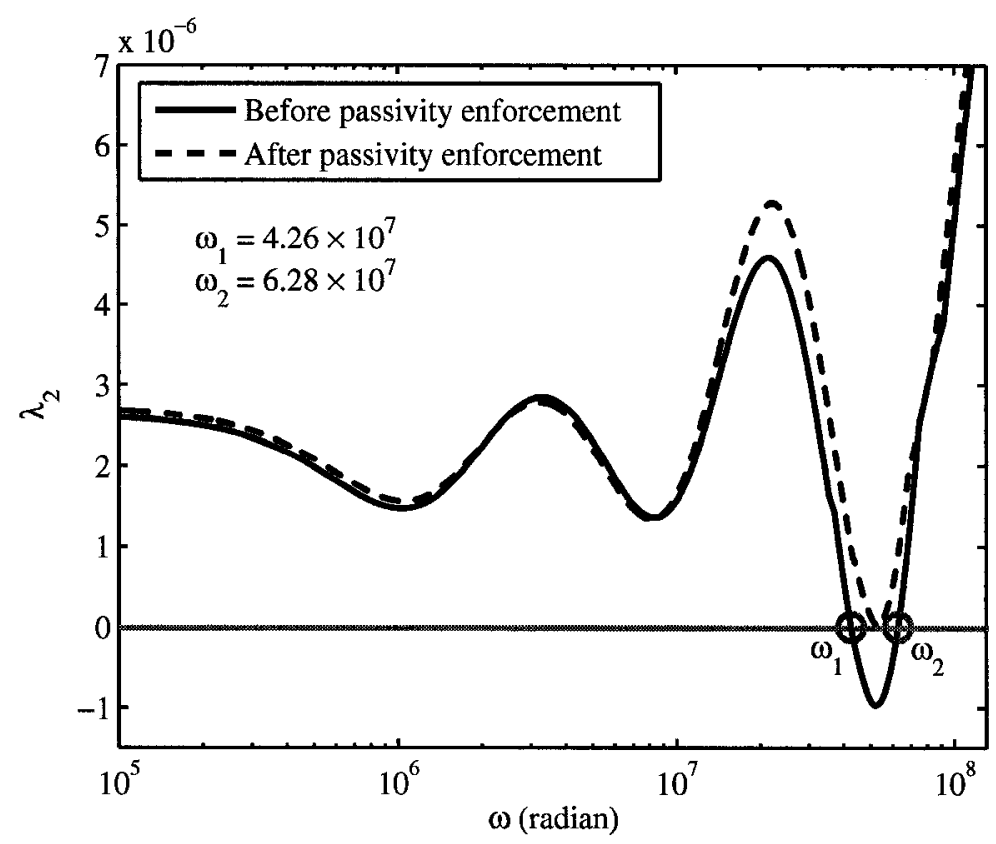

Figure 5.11: Eigenvalue spectrum of $\Re\{\tilde{\boldsymbol{Y}}(s)\}, \lambda_{2}$ (Example 3).

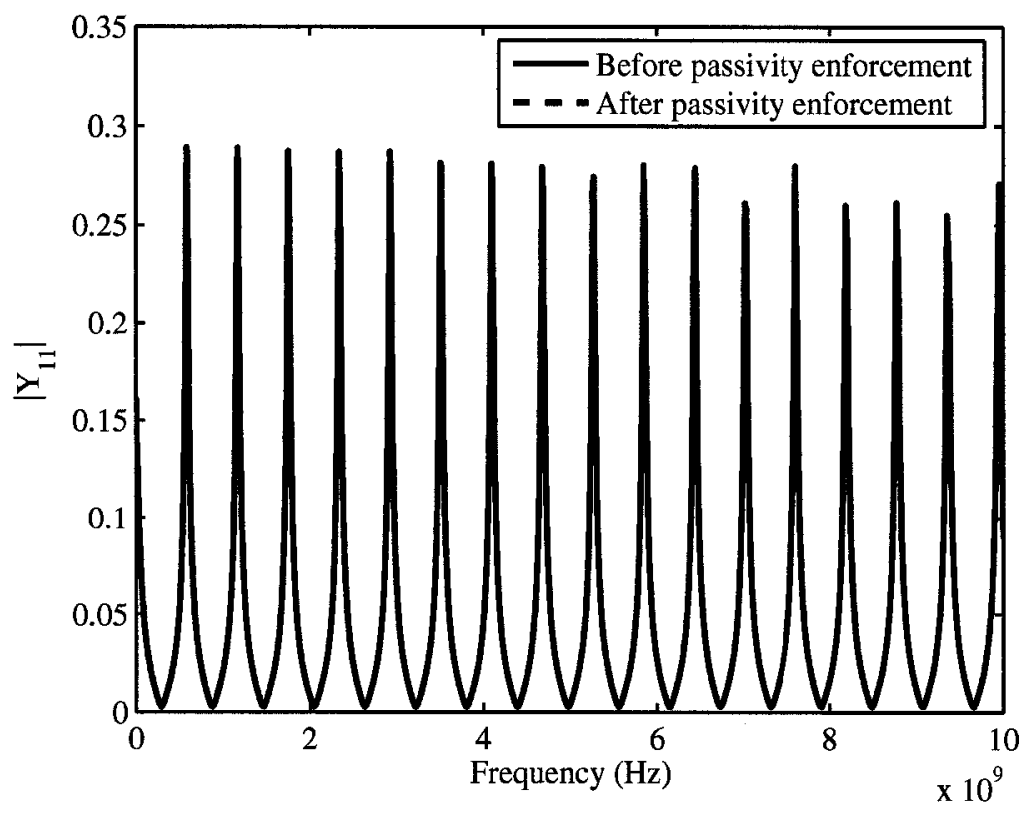

Figure 5.12: Comparison of frequency responses, $\left|\boldsymbol{Y}_{11}\right|$ (Example 3). 


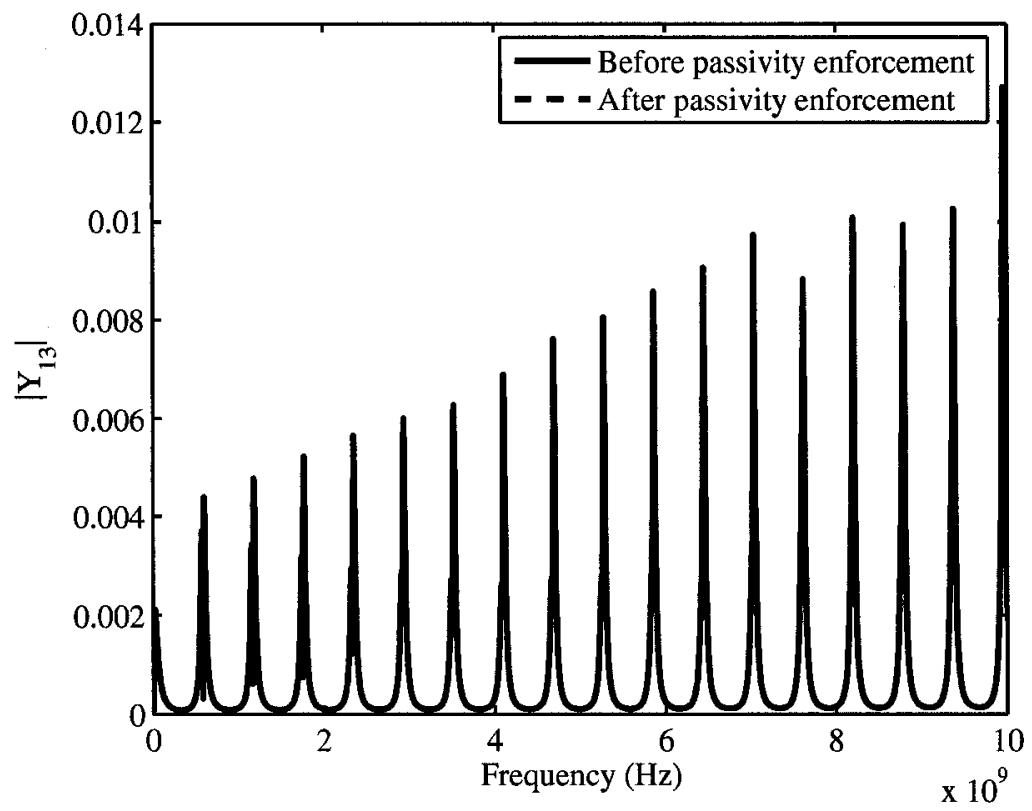

Figure 5.13: Comparison of frequency responses, $\left|\boldsymbol{Y}_{13}\right|$ (Example 3).

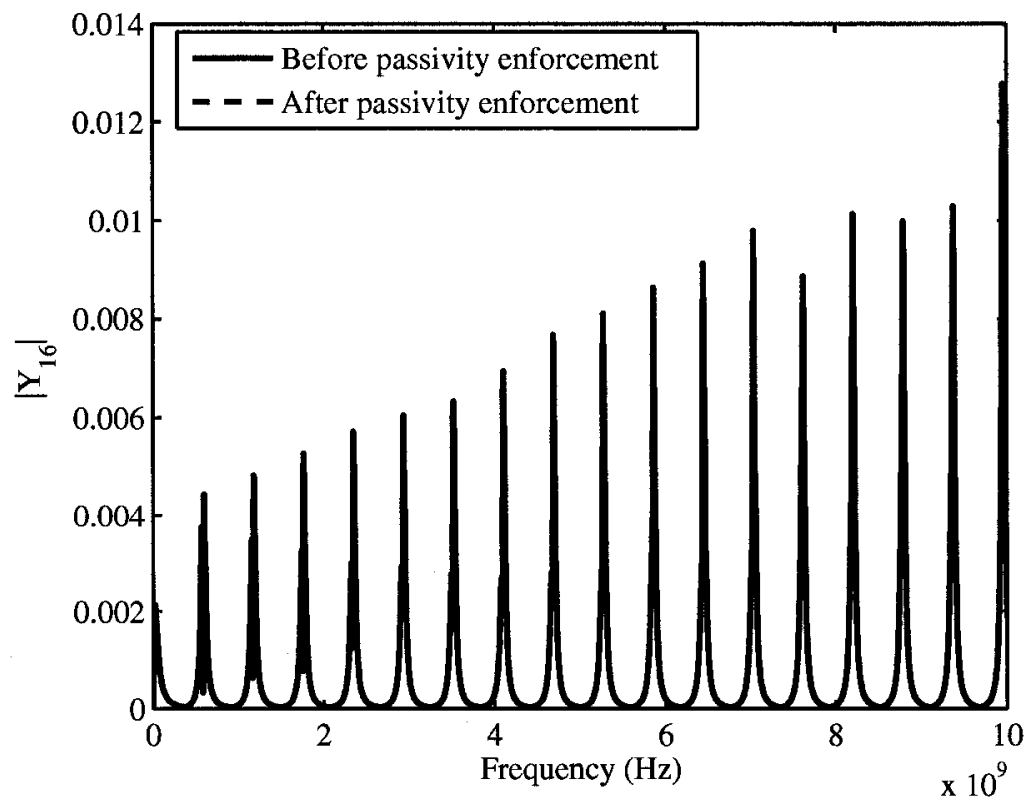

Figure 5.14: Comparison of frequency responses, $\left|\boldsymbol{Y}_{16}\right|$ (Example 3). 


\section{Chapter 6}

\section{Circuit Modeling for FD-PUL Parameters}

\subsection{Introduction}

The PUL parameters used to describe a transmission line are typically obtained from measurements, empirical formulas or electromagnetic simulation and presented at discrete frequency points. As the system operating frequency increases up to Giga-Hertz range, these parameters exhibit certain phenomena versus frequency due to proximity, edge and skin effects, and the change of properties of the dielectric materials etc. [2,4,25]. Hence they can no longer be deemed as frequency independent. As an example, at high frequencies, the edge effects cause the currents in the TL conductors to be concentrated toward the sharp edges. This in turn increases the resistance and inductance. In other words, the PUL parameters are likely frequency dependent. An illustration of the frequency variation of the PUL parameters of a stripline is given in Figure 6.1 [4]. At low frequencies, the parameters are almost constant. As the frequency goes higher, the PUL resistance, $R$, and conductance, $G$, increase, while the PUL inductance, $L$, and capacitance, $C$, decrease. It has been shown in the literature $[2,4]$ that the PUL resistance is nearly proportional to 


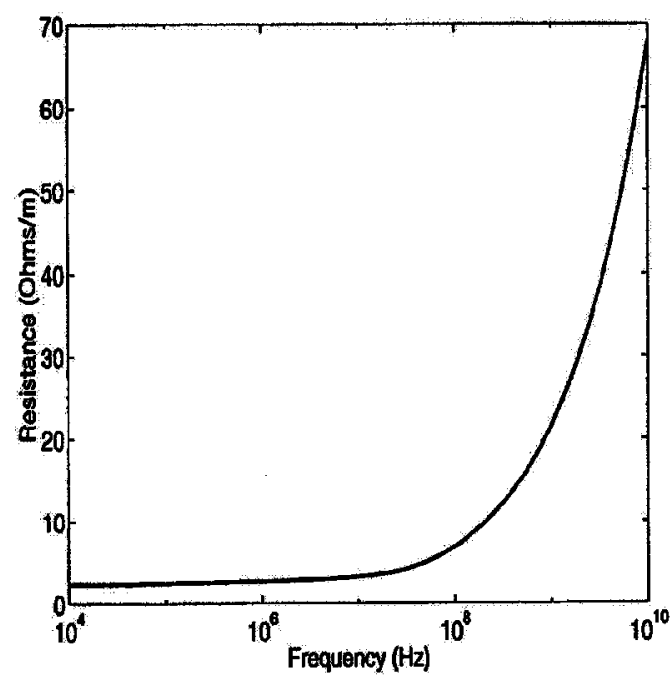

(a) PUL resistance, $\boldsymbol{R}$, versus frequency.

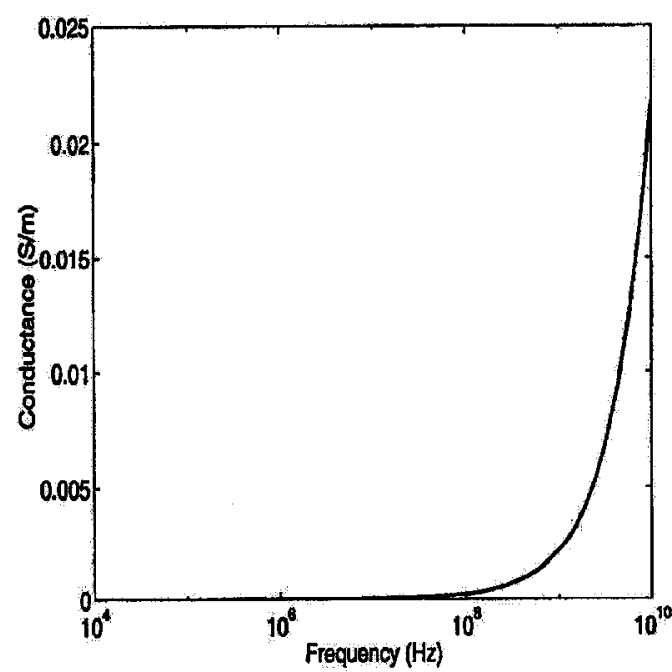

(c) PUL conductance, $\boldsymbol{G}$, versus frequency.

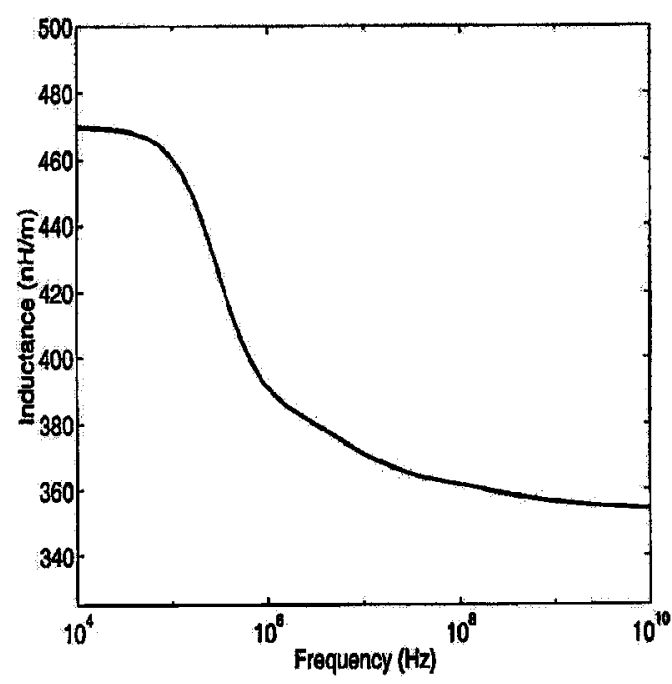

(b) PUL inductance, $L$, versus frequency.

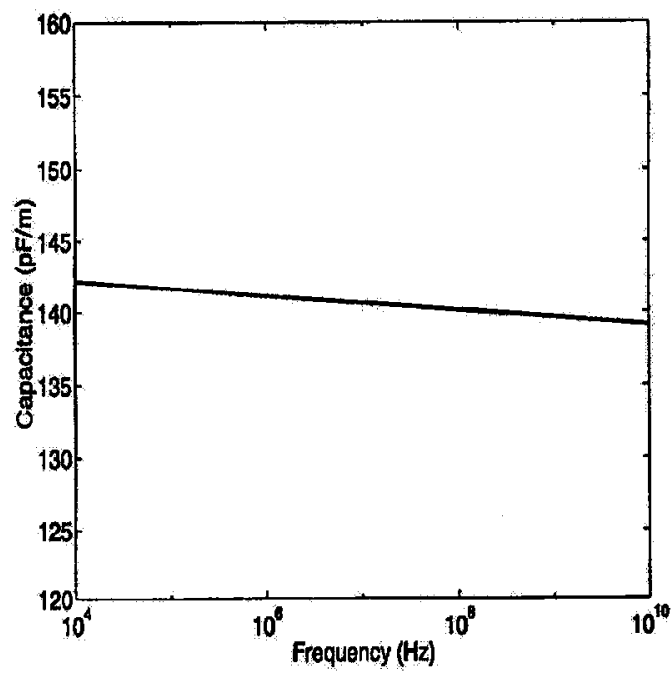

(d) PUL capacitance, $C$, versus frequency.

Figure 6.1: An example of frequency-dependent variation of the PUL parameters. 
the square root of the frequency at high frequencies. The inductance is proportional to the inverse of the square root of frequency and approaches a constant value at large frequencies, while the PUL capacitance decreases slightly at high frequencies. The dependency of the PUL parameters on frequency presents various difficulties for many macromodeling and MOR techniques for the TLs. For example, the RLC components in the series lumped model would become frequency-dependent, which cannot be accepted by generic circuit simulators, such as SPICE.

One popular approach to handle the FD-PUL parameters is based on approximating the PUL impedance $\mathcal{Z}(s)=\boldsymbol{R}(s)+s \boldsymbol{L}(s)$ and the PUL admittance $\mathcal{Y}(s)=\boldsymbol{G}(s)+s \boldsymbol{C}(s)$ with two lumped networks, whose driving impedance and admittance are close to $\mathcal{Z}(s)$ and $\mathcal{Y}(s)$, respectively [23,25, 103-105]. MRA [105] achieves this by fixing a network topology (such as resistor-inductor (RL) canonical or conductor-capacitor (GC) Fork-like network) and then optimizing the value of each component in the topology. One advantage of this scheme is that the approximated network is guaranteed to be passive by forcing each component value to be greater than zero. However, there is not much freedom in controlling the approximation. PRIME [104] obtains the approximation for rational functions based on Vectfit technique [61]. The fitted rational function are then synthesized with lumped networks. Vectfit is efficient for the approximation and can produce stable rational functions. However, it usually also produces complex poles and there is no guarantee of the PR-ness of the approximation. Unfortunately, circuit synthesis for rational functions with complex poles is a complicated task $[106,107]$. Meanwhile, PR-ness is an important property that needs to be preserved in the PUL parameters. The algorithm in [60] uses a guaranteed passive modeling approach based on convex programming to approximate a general network characterized by discrete frequency measurements. Although this approach can be used to approximate the PUL parameter matrices with PR rational functions, it is a relatively 
computationally expensive approach for handling the mild frequency behavior exhibited by those matrices.

This chapter presents a robust rational approximation scheme for the PUL parameters, as well as efficient network synthesis topologies for PR rational functions. The proposed algorithm can optimally achieve the approximation while meeting the desirable degree of accuracy. The resulting rational function has real poles only, thereby making the synthesis process simpler. The development of the proposed algorithm is inspired by the following observations [108]:

1. For most practical cases, the behavior of the PUL parameters, $\mathcal{Z}(s)$ and $\mathcal{Y}(s)$, is a monotonic function of frequency, and can be easily characterized with resistorinductor and resistor-capacitor (RC) networks, respectively.

2. For a multi-port RL (RC) network, the mutual terms $\mathcal{F}_{j k}$ contain only those poles which are present both in $\mathcal{F}_{j j}$ and $\mathcal{F}_{k k}$. Here $\mathcal{F}$ represents either $\mathcal{Z}(s)$ or $\mathcal{Y}(s)$, and $\mathcal{F}_{i j}$ is its $(i, j)$ entry. The poles must lie on the negative real axis.

The rest of this chapter is organized as follows. Section 6.2 describes the proposed rational approximation scheme for the frequency-dependent PUL parameters. Section 6.3 discusses the lumped network synthesis and a newly proposed network synthesis based on Laplace elements for the rational functions. Section 6.4 presents some numerical examples based on the proposed algorithm. It is shown that a good approximation accuracy can be obtained for the FD-PUL parameters with low order rational functions. The Laplace elements based network synthesis is also demonstrated to be more efficient and robust than lumped network based synthesis. 


\subsection{Development of the proposed modeling algorithm}

The basic PUL parameters for a physical transmission line are non-negative definite matrices at each frequency point $[2,109]$. Other than accuracy and efficiency, passivity is an important property needed to be preserved during the modeling process. For this purpose, the approximated rational function is required to be positive-real. This section lays out the process of the proposed algorithm in two subsections. Subsection 6.2.1 discusses the formulation of the approximation. Subsection 6.2.2 presents the formulation of the error function that is to be minimized during approximation. The positive-realness in the resulting rational functions is also discussed.

\subsubsection{Formulation of the approximation}

Given that the PUL impedance and admittance parameters are provided at discrete frequency points, $\mathcal{Z}\left(s_{k}\right), \mathcal{Y}\left(s_{k}\right), k=1,2, \ldots, K$, the following form of rational function is adopted in the approximation.

$$
\mathcal{Z}(s), \mathcal{Y}(s) \approx \tilde{\mathcal{Z}}(s), \tilde{\mathcal{Y}}(s)=\mathbb{D}+s \mathbb{E}+\sum_{n=1}^{N}\left[\frac{s \mathbb{K}_{n}}{s-p_{n}}+\frac{\mathbb{F}_{n}}{s-p_{n}}\right]
$$

where $\mathbb{D}, \mathbb{E}, \mathbb{K}_{n}$ and $\mathbb{F}_{n}$ are $m \times m$ matrices for a $(m+1)$-conductor transmission line. In the context of this thesis, they are called constant term, linear term, $\mathbb{K}$-residue and $\mathbb{F}$-residue, respectively. $p_{n}$ denotes the poles of the rational function. The goal of the approximation is then seeking $p_{n}, \mathbb{D}, \mathbb{E}, \mathbb{K}_{n}$ and $\mathbb{F}_{n}$ so that the error between $\mathcal{Z}(s), \mathcal{Y}(s)$ and $\tilde{\mathcal{Z}}(s), \tilde{\mathcal{Y}}(s)$ is minimized. According to the two observations stated in Section 6.1, $p_{n}$ 's are pre-selected to be some negative values distributed within $-\left[f_{\min }, f_{\max }\right]$ in logarithmic scale. Here, $\left[f_{\min }, f_{\max }\right]$ denotes the frequency range of interest. By doing this, the rational function is always stable. $\mathbb{D}$ is chosen to be the PUL resistance for $\mathcal{Z}(s)$ (or conductance for $\mathcal{Y}(s)$ ) at zero frequency point. $\mathbb{E}$ is chosen to be the PUL inductance for $\mathcal{Z}(s)$ (or capacitance 
for $\mathcal{Y}(s))$ at $s \rightarrow \infty$. The determination of $\mathbb{K}_{n}$ and $\mathbb{F}_{n}$ is through solving the minimization equation stated in the following subsection.

\subsubsection{Error criteria and positive-realness}

Provided that $p_{n}, \mathbb{D}$ and $\mathbb{E}$ have been specified as shown in Subsection 6.2.1, for each element of $\mathcal{Z}(s)$ and $\mathcal{Y}(s)$, say the $(i, j)^{\text {th }}$ element ${ }^{1}$, substituting the original data into (6.1) will result in $K$ equations, corresponding to $K$ frequency points. The conventional least mean square (LMS) scheme can be used to solve these $K$ equations for $\mathbb{K}_{n}$ and $\mathbb{F}_{n}$. The problem of LMS is then formulated as follows, ${ }^{2}$,

$$
\min \sum_{k=1}^{K}\left\|\tilde{\mathcal{Z}}_{i, j}\left(s_{k}\right)-\mathcal{Z}_{i, j}\left(s_{k}\right)\right\|^{2}, \quad \text { or } \min \sum_{k=1}^{K}\left\|\tilde{\mathcal{Y}}_{i, j}\left(s_{k}\right)-\mathcal{Y}_{i, j}\left(s_{k}\right)\right\|^{2} .
$$

Equation (6.4) minimizes the summation of the absolute error at each frequency point. This is reasonable when the magnitude of the function under consideration is of a similar order at different frequency points. However, the PUL $\mathcal{Z}(s)$ and $\mathcal{Y}(s)$ usually have a much larger magnitude at high frequencies than at low frequencies. Using (6.4) may result in an unbalanced approximation at low frequency, since the error at high frequency can easily dominate the total error. To address this issue, the methodology of minimizing the relative error is adopted, which is stated below,

$$
\min \sum_{k=1}^{K} \frac{w_{k}\left\|\tilde{\mathcal{Z}}_{i, j}\left(s_{k}\right)-\mathcal{Z}_{i, j}\left(s_{k}\right)\right\|^{2}}{\left\|\mathcal{Z}_{i, j}\left(s_{k}\right)\right\|^{2}}, \quad \text { OR } \min \sum_{k=1}^{K} \frac{w_{k}\left\|\tilde{\mathcal{Y}}_{i, j}\left(s_{k}\right)-\mathcal{Y}_{i, j}\left(s_{k}\right)\right\|^{2}}{\left\|\mathcal{Y}_{i, j}\left(s_{k}\right)\right\|^{2}}
$$

\footnotetext{
${ }^{1}$ The matrix structures of $\mathcal{Z}(s)$ and $\mathcal{Y}(s)$ are depicted as:

$$
\mathcal{Z}(s)=\left[\mathcal{Z}_{i, j}(s)\right], \quad \mathcal{Y}(s)=\left[a_{i, j} \mathcal{Y}_{i, j}(s)\right], \quad i, j=1, \ldots, m
$$$$
\text { where, } a_{i, j}=1 \text { if } i=j ; a_{i, j}=-1 \text { if } i \neq j \text {. }
$$

${ }^{2}$ The matrix structures of $\tilde{\mathcal{Z}}(s)$ and $\tilde{\mathcal{Y}}(s)$ are depicted as:

$$
\tilde{\mathcal{Z}}(s)=\left[\tilde{\mathcal{Z}}_{i, j}(s)\right], \quad \tilde{\mathcal{Y}}(s)=\left[a_{i, j} \tilde{\mathcal{Y}}_{i, j}(s)\right], \quad i, j=1, \ldots, m
$$

where, $a_{i, j}=1$ if $i=j ; a_{i, j}=-1$ if $i \neq j$.
} 
In (6.5), a weight, $w_{k}$, is assigned to each frequency point to add more freedom of control on the approximation.

The minimization in (6.5) does not contain any constraints on the positive-realness. This would speed up the approximation process. But the resulting rational function is not guaranteed to be PR. The PR-ness of the approximated rational functions is checked and compensated using the technique proposed in [110]. For most cases, the direct outputs of the rational approximation are PR. Hence, the passivity or PR-ness compensation is not required. It is worth noting that passivity compensation step can be accomplished easily here, in case it needs to be done, because of the simple structure of the rational functions, which include only real poles with low orders.

In the implementation, the proposed algorithm starts with one-pole approximation, and increases the number of poles by one at each iteration. The iteration is stopped, or the approximation is accepted, when $\left\|\tilde{\mathcal{Z}}_{i, j}(s)\right\|$ and $\left\|\tilde{\mathcal{Y}}_{i, j}(s)\right\|$ are close enough to $\left\|\mathcal{Z}_{i, j}(s)\right\|$ and $\left\|\mathcal{Y}_{i, j}(s)\right\|$, respectively, within a user defined criteria at certain number of frequency points. For most of the practical cases, we found that very few poles are enough to achieve the desired accuracy.

\subsection{Network synthesis}

This section explains the synthesis of the rational functions into lumped networks, followed by presenting a new network synthesis methodology based on Laplace elements.

\subsubsection{Realization of the PUL networks using lumped components}

Given that the PUL $\mathcal{Z}(s)$ and $\mathcal{Y}(s)$ have, in respective order, been approximated with a $N_{z}$ and $N_{y}$ order rational function with real poles, an RL-RC tank-type network, shown in Figure 6.2, can be used to synthesize each element of $\tilde{\mathcal{Z}}(s)$ into a lumped network. 
Similarly, a GC-GL Foster-type network, shown in Figure 6.3, can be used to synthesize each element of $\tilde{\mathcal{Y}}(s)$ into a lumped network.

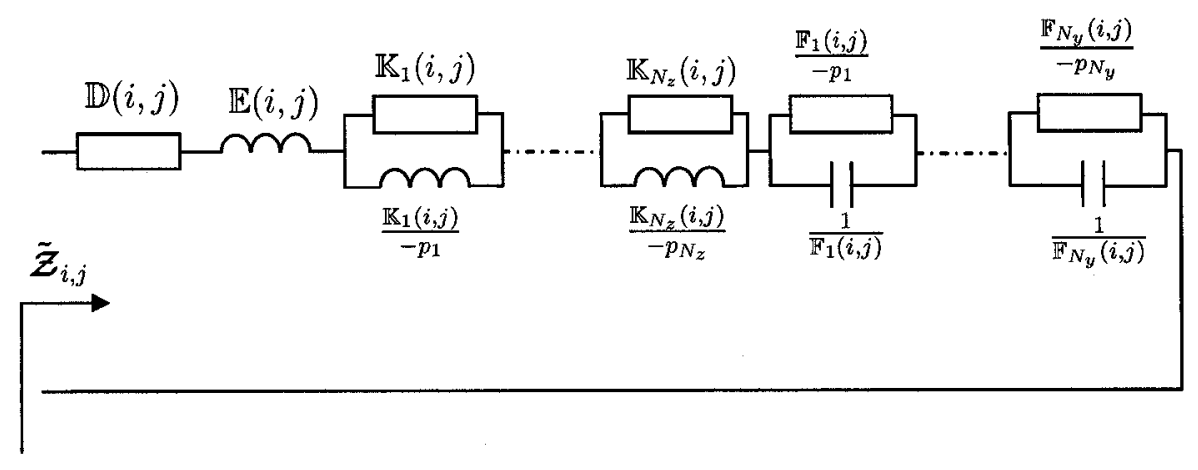

Figure 6.2: Impedance topology in approximating $\mathcal{Z}_{i j}$.

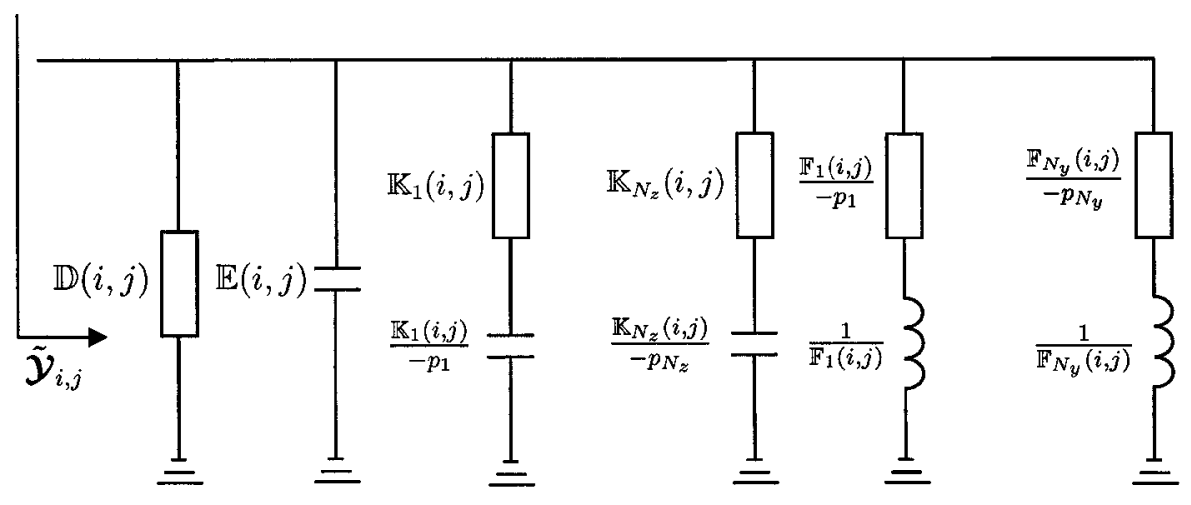

Figure 6.3: Admittance topology in approximating $\mathcal{Y}_{i j}$.

After the synthesized networks have been obtained for each element of $\tilde{\mathcal{Z}}(s)$ and $\tilde{\mathcal{Y}}(s)$, the complete model network for the whole matrices can follow the topologies in [111], which are re-depicted in Figures 6.4 and 6.5 for a four-conductor TL for illustration. In the figures,

$$
\hat{\mathcal{Z}}_{i, i}=\tilde{\mathcal{Z}}_{i, i}-\sum_{k=1, k \neq i}^{m} \tilde{\mathcal{Z}}_{i, k}, \quad \hat{\mathcal{Y}}_{i, i}=\tilde{\mathcal{Y}}_{i, i}-\sum_{k=1, k \neq i}^{m} \tilde{\mathcal{Y}}_{i, k}, \quad\left\{\begin{array}{l}
i=1, \ldots, m \\
m=3
\end{array}\right.
$$

Each block in the figures is replaced by the RL-RC tank or GC-GL Foster-like networks generated in Figures 6.2 and 6.3. 


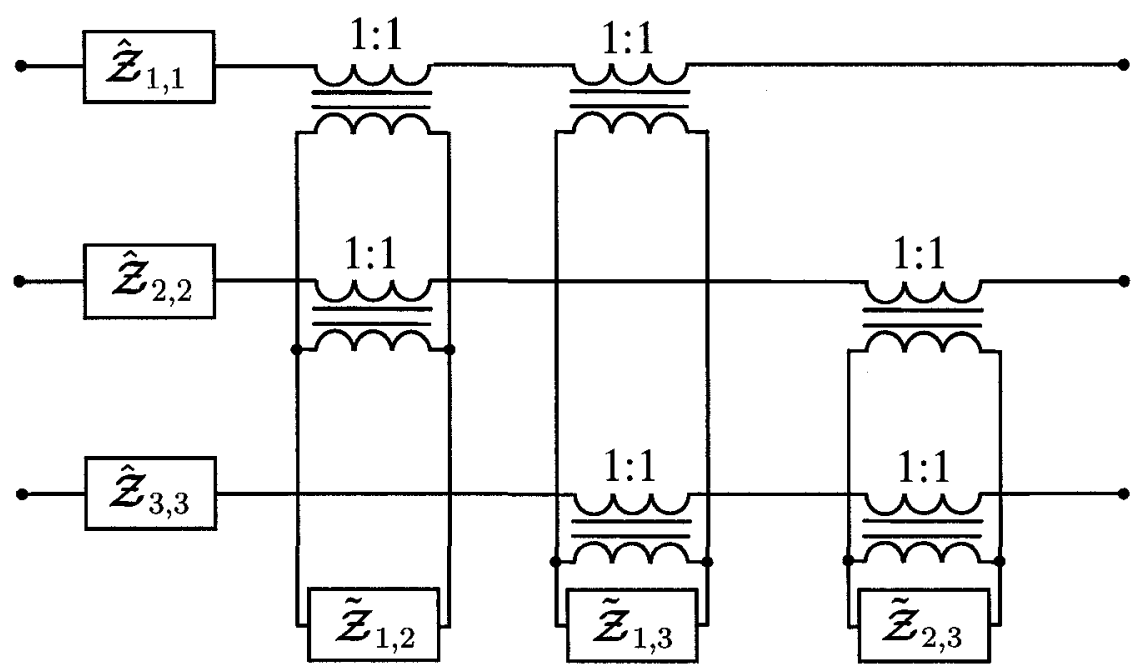

Figure 6.4: Lumped network realization for $\tilde{\mathcal{Z}}(s)$ of a four-conductor TL.

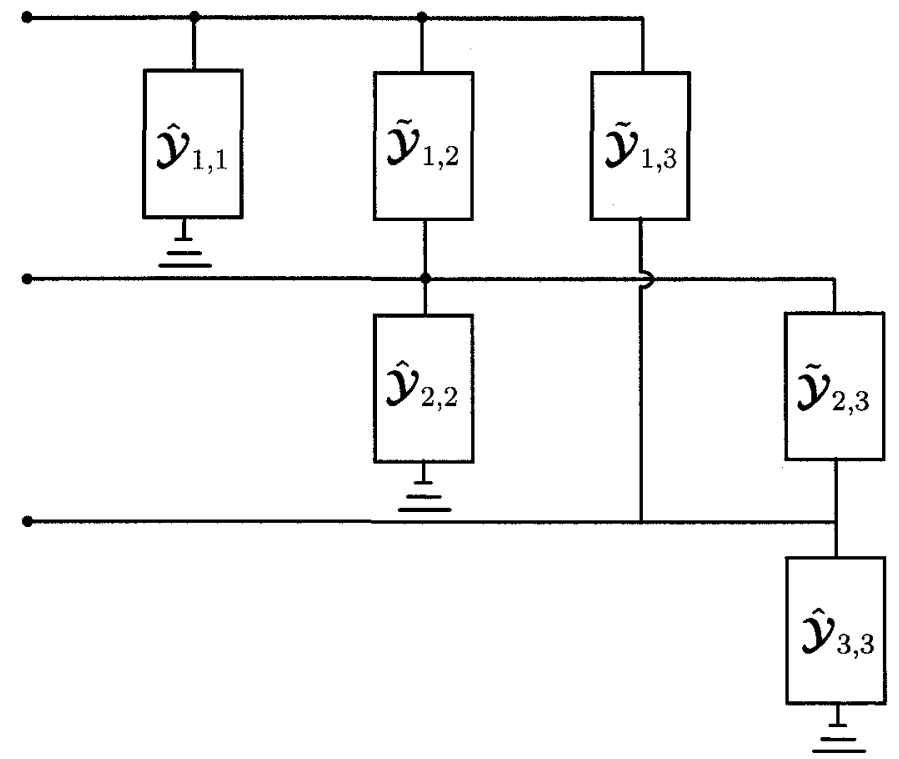

Figure 6.5: Lumped network realization for $\tilde{\mathcal{Y}}(s)$ of a four-conductor TL. 
It is noted that a direct synthesis on each element of $\tilde{\mathcal{Z}}(s)$ and $\tilde{\mathcal{Y}}(s)$ in the way depicted in Figures 6.2 and 6.3 may result in some impractical (such as negative values, huge or very small values) lumped components. To illustrate this issue, we consider a PR matrix function in (6.7) approximating a PUL impedance.

$$
\tilde{\mathcal{Z}}(s)=\left[\begin{array}{ll}
\tilde{\mathcal{Z}}_{11}(s) & \tilde{\mathcal{Z}}_{12}(s) \\
\tilde{\mathcal{Z}}_{21}(s) & \tilde{\mathcal{Z}}_{22}(s)
\end{array}\right]
$$

Here, the values obtained by solving (6.5) are as follows,

$$
\begin{gathered}
\tilde{\mathcal{Z}}_{11}(s)=\tilde{\mathcal{Z}}_{22}(s)=2.84 \times 10^{-14}+s \times 1.69 \times 10^{-9}+\frac{s \times 141.8}{s+6.28 \times 10^{8}} \\
+\frac{8.51 \times 10^{10}}{s+6.28 \times 10^{8}}+\frac{s \times 164.2}{s+3.14 \times 10^{10}} \\
\tilde{\mathcal{Z}}_{21}(s)=\tilde{\mathcal{Z}}_{12}(s)=-s \times 1.20 \times 10^{-9}+\frac{s \times 4.02}{s+6.28 \times 10^{8}} \\
+\frac{5.61 \times 10^{8}}{s+6.28 \times 10^{8}}+\frac{s \times 186.3}{s+3.14 \times 10^{10}}
\end{gathered}
$$

Direct synthesis of $\tilde{\mathcal{Z}}_{11}(s)$ (or $\tilde{\mathcal{Z}}_{22}(s)$ ) would produce a network shown in Figure 6.6. In this network, there is one very small resistor. Similarly, Figure 6.7 shows the network from the direct synthesis for $\tilde{\mathcal{Z}}_{12}(s)$ (or $\tilde{\mathcal{Z}}_{21}(s)$ ), where there exist one negative inductor. One reason incurring these impractical elements is that the rational functions for the offdiagonal elements of $\tilde{\mathcal{Z}}(s)$ and $\tilde{\mathcal{Y}}(s)$ are not guaranteed to be positive-real even if the whole matrix function is PR. Including a network in the presence of these impractical elements in a transient simulation may cause numerical problems, such as "Time step too small" error in HSPICE ${ }^{\circledR}$.

To avoid these problems, a new network synthesis based on Laplace elements is proposed and presented in the next subsection (Section 6.3.2). It will also be demonstrated by the numerical examples presented in Section 6.4 that the network realized using this new scheme, when included in transient simulation, is more efficient than the network realized 


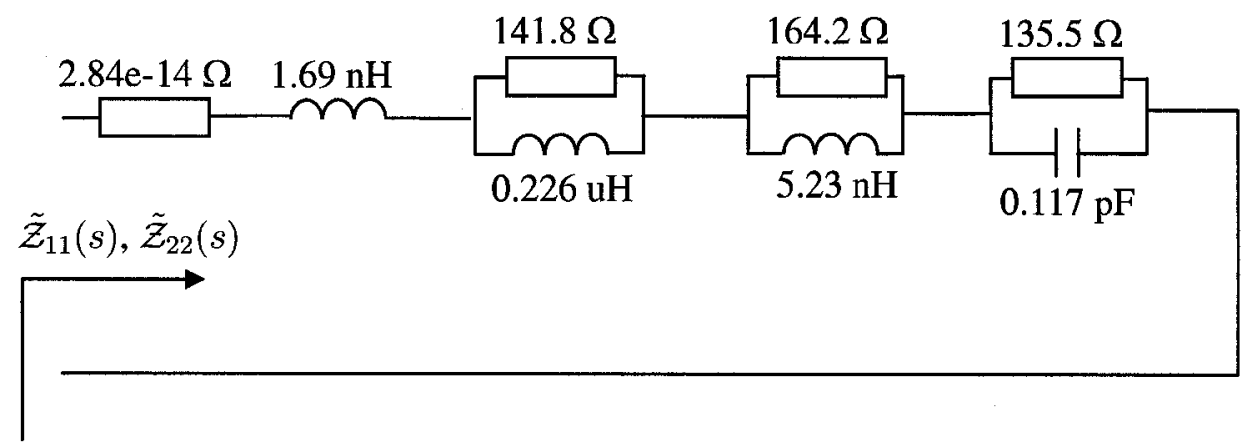

Figure 6.6: Network synthesis for $\tilde{\mathcal{Z}}_{11}(s)$ and $\tilde{\mathcal{Z}}_{22}(s)$ of the example impedance.

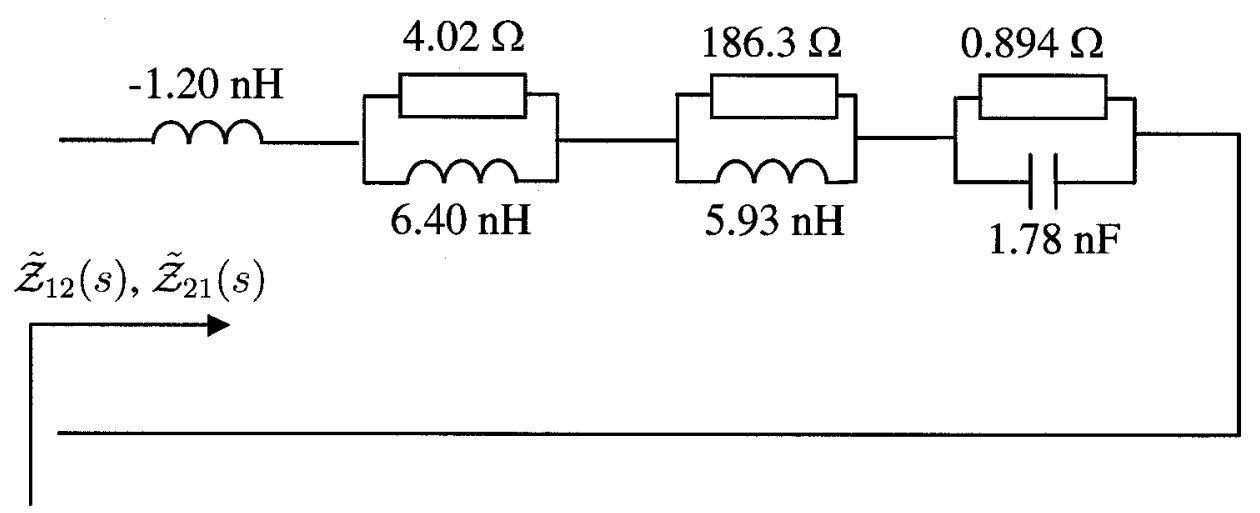

Figure 6.7: Network synthesis for $\tilde{\mathcal{Z}}_{12}(s)$ and $\tilde{\mathcal{Z}}_{21}(s)$ of the example impedance. 
based on Figures 6.2-6.5.

\subsubsection{Realization of the PUL networks using Laplace elements}

The terminology, 'Laplace element', is borrowed from HSPICE ${ }^{\circledR}$. It is used to denote a circuit component, whose constitutive equation is represented by a rational function in the Laplace domain. In HSPICE ${ }^{\circledR}$, there are two Laplace elements: " $G$ " element and "E" element, representing voltage controlled current sources and voltage controlled voltage sources, respectively. In other circuit simulators, there are similar elements, such as "svcvs" in Virtuoso Spectre ${ }^{\circledR}$.

Given that the PUL impedance and admittance matrices have been approximated by PR matrix functions $\tilde{\mathcal{Z}}(s)$ and $\tilde{\mathcal{Y}}(s)$, respectively, let,

$$
\left[\begin{array}{c}
V_{1} \\
\vdots \\
V_{i} \\
\vdots \\
V_{m}
\end{array}\right]=\tilde{\mathcal{Z}}(s)\left[\begin{array}{c}
I_{1} \\
\vdots \\
I_{i} \\
\vdots \\
I_{m}
\end{array}\right]=\left[\begin{array}{ccccc}
\tilde{\mathcal{Z}}_{1,1} & \ldots & \ldots & \ldots & \tilde{\mathcal{Z}}_{1, m} \\
\vdots & & & & \vdots \\
\vdots & & \tilde{\mathcal{Z}}_{i, j} & & \vdots \\
\vdots & & & & \vdots \\
\tilde{\mathcal{Z}}_{m, 1} & \ldots & \ldots & \ldots & \tilde{\mathcal{Z}}_{m, m}
\end{array}\right]\left[\begin{array}{c}
I_{1} \\
\vdots \\
I_{i} \\
\vdots \\
I_{m}
\end{array}\right]
$$

where $\left(V_{1}, I_{1}\right), \ldots,\left(V_{m}, I_{m}\right)$ denote the $m$ pairs of port voltages and currents of the PUL impedance network. Expanding the equations in (6.10), we have

$$
V_{i}=\sum_{j=1, j \neq i}^{m} \tilde{\mathcal{Z}}_{i, j} I_{j}+\tilde{\mathcal{Z}}_{i, i} I_{i}=\sum_{j=1, j \neq i}^{m} \frac{\tilde{\mathcal{Z}}_{i, j}}{\tilde{\mathcal{Z}}_{j, j}} \bar{V}_{j}+\bar{V}_{i}
$$

where $\bar{V}_{i}=\tilde{\mathcal{Z}}_{i, i} I_{i}$ and $\bar{V}_{j}=\tilde{\mathcal{Z}}_{j, j} I_{j}$ represent the voltage in series with port $i$ and $j$, respectively, which are resulted from self-impedance. Equation (6.11) shows that the new network model for $\tilde{\mathcal{Z}}(s)$ based on Laplace elements can be constructed as in Figure 6.8, which can be embedded in circuit simulators for simulation. As an example, the diagonal blocks can be realized with "G" elements and the off-diagonal blocks can be realized with 
"E" elements in HSPICE ${ }^{\circledR}$

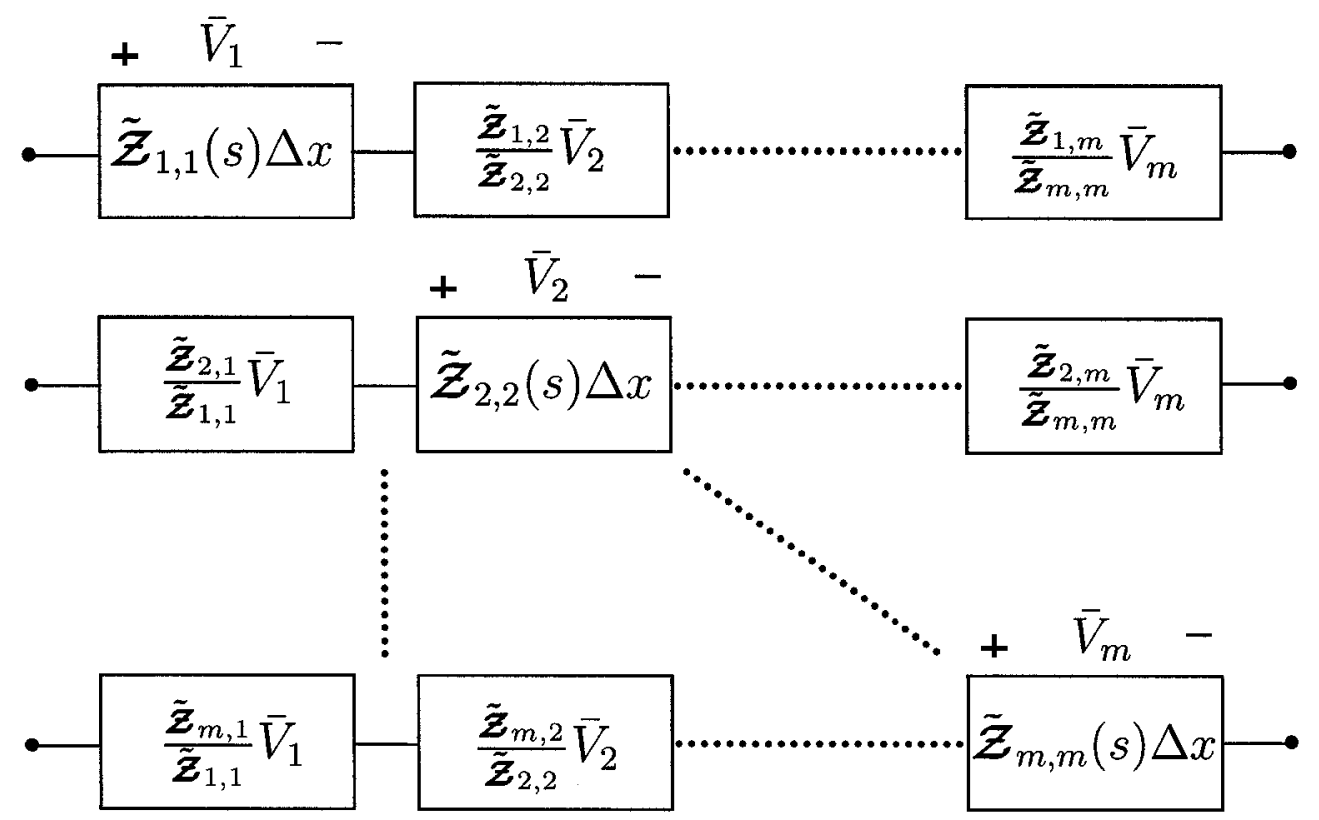

Figure 6.8: Network synthesis for $\tilde{\mathcal{Z}}(s)$ based on Laplace elements.

Similarly, let

$$
\left[\begin{array}{c}
I_{1} \\
\vdots \\
I_{i} \\
\vdots \\
I_{m}
\end{array}\right]=\tilde{\mathcal{Y}}(s)\left[\begin{array}{c}
V_{1} \\
\vdots \\
V_{i} \\
\vdots \\
V_{m}
\end{array}\right]=\left[\begin{array}{ccccc}
\tilde{\mathcal{Y}}_{1,1} & \ldots & \ldots & \ldots & -\tilde{\mathcal{Y}}_{1, m} \\
\vdots & & & & \vdots \\
\vdots & & a_{i, j} \tilde{\mathcal{Y}}_{i, j} & & \vdots \\
\vdots & & & & \vdots \\
\tilde{\mathcal{Y}}_{m, 1} & \ldots & \ldots & \ldots & \tilde{\mathcal{Y}}_{m, m}
\end{array}\right]\left[\begin{array}{c}
V_{1} \\
\vdots \\
V_{i} \\
\vdots \\
V_{m}
\end{array}\right]
$$

where $\left(V_{1}, I_{1}\right), \ldots,\left(V_{m}, I_{m}\right)$ denote the $m$ pairs of port voltages and currents of the PUL admittance network. Expanding the equations in (6.12), we have

$$
\begin{aligned}
I_{i} & =\sum_{j=1}^{m} a_{i, j} \tilde{\mathcal{Y}}_{i, j} V_{j}=\sum_{j=1, j \neq i}^{m}-\tilde{\mathcal{Y}}_{i, j} V_{j}+\mathbb{D}_{i, i} V_{i}+\left(\tilde{\mathcal{Y}}_{i, i}-\mathbb{D}_{i, i}\right) V_{i} \\
& =\sum_{j=1, j \neq i}^{m} I_{i, j}+\mathbb{D}_{i, i} V_{i}+\hat{I}_{i, i}
\end{aligned}
$$

where $I_{i, j}=-\tilde{\mathcal{Y}}_{i, j} V_{j}, \hat{I}_{i, i}=\left(\tilde{\mathcal{Y}}_{i, i}-\mathbb{D}_{i, j}\right) V_{i}$ and $\mathbb{D}_{i, i}$ denotes the $(i, i)^{\text {th }}$ element of $\mathbb{D}$ 
matrix (constant term). Equation (6.14) shows that the new network model for $\tilde{\mathcal{Y}}(s)$ based on Laplace elements can be constructed as in Figure 6.9. All current sources in the figure can be realized with " $G$ " elements in HSPICE ${ }^{\circledR}$.
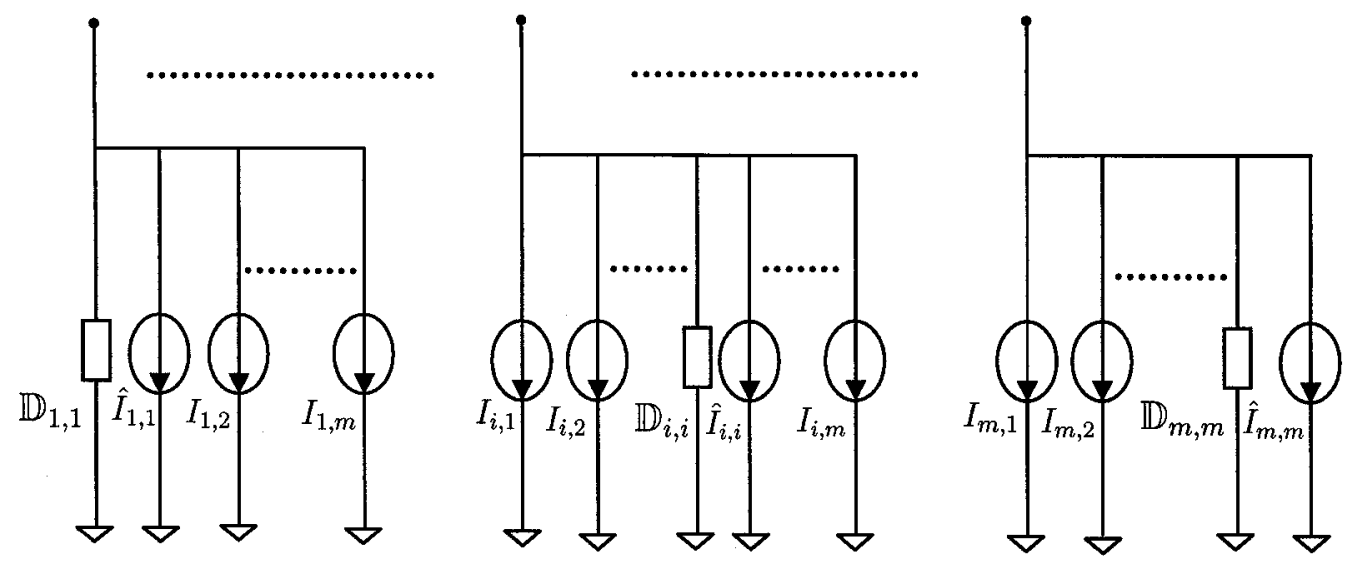

Figure 6.9: Network synthesis for $\tilde{\mathcal{Y}}(s)$ based on Laplace elements.

\subsection{Numerical examples}

The proposed algorithm was implemented and tested for the numerical performance using three TL examples. In each example, the PUL impedance and admittance were first approximated by two positive-real rational functions, which were then synthesized into equivalent networks using both methodologies described in the previous section. In order to compare the performance of those two network synthesis methodologies, the transmission line was modeled using the series lumped model and embedded in a circuit network for transient simulation. The reference used for transient responses validation was obtained from inverse fast fourier transform (IFFT). The curves of this reference were labeled as " $\mathrm{xx}$ - IFFT" when plotted in the figures. 


\subsubsection{Example 1}

In this example, a 3-conductor transmission line with a length of $5 \mathrm{~cm}$ was considered. The variation of PUL $R, L, G$ and $C$ parameters versus frequency are defined in [85], where it is denoted as Line 6. The transmission line was embedded in a network as shown in Figure 6.10, which was driven by a pulse current source.

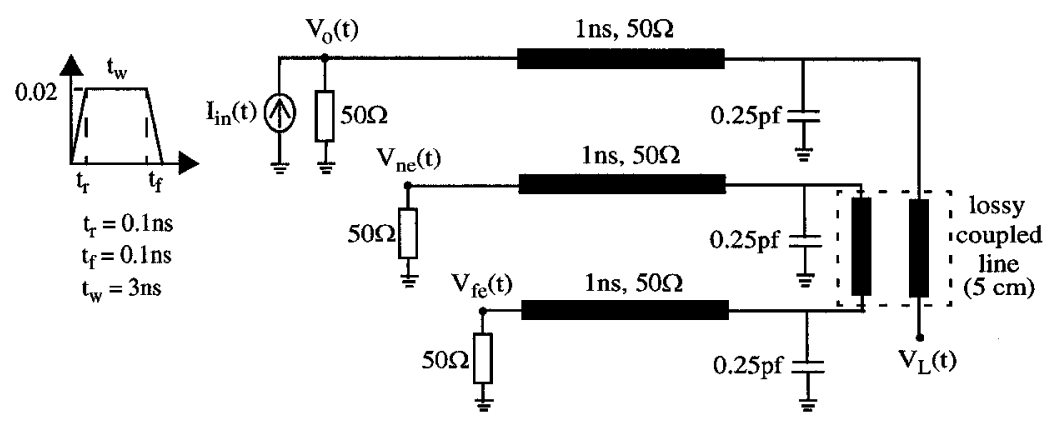

Figure 6.10: Circuit topology for Example 1.

In order to validate the proposed algorithm, the PUL impedance and admittance were first approximated using a fourth-order and a third-order rational function, respectively. The approximated rational function were PR. Figure 6.11 plotted the comparison between the approximated functions and the original data points. They matched very well.

The approximated rational functions were then synthesized into one lumped PUL impedance network and one lumped PUL admittance network. The RL-RC-Tank-type network used to synthesize the impedance functions consisted of one resistor in series with one inductor and four $R L$ tanks. The GC-GL-Foster-type network used to synthesize the admittance function included one conductor in parallel with one capacitor and three $G C$ branches. Further, the resulted PUL networks were used to constructed a series lumped model with 40 sections for the TL. Based on the series lumped model, the transient simulation was performed on the circuit shown in Figure 6.10. Figure 6.12 shows the transient responses (labeled as "xx - lump") at different nodes in the circuit. It could be observed 
that the responses from lumped model were in good agreement with the reference (i.e. IFFT, shown in solid line).

To provide a comparison between the Laplace element-based network synthesis with the above lumped element-based synthesis, another series lumped model with 40 sections was constructed for the TL and plugged into the circuit for re-simulation. However, this time the PUL networks used to build the series lumped network were based on the new models proposed in Figures 6.8 and 6.9. The transient responses based on these new models were also plotted in Figure 6.12 (labeled as "xx - laplace"). As expected, they were on top of the responses based on the lumped PUL networks, since, in both cases, the system functions for the PUL networks were the same.

\subsubsection{Example 2}

In this example, a transmission line with three conductors of a length $10 \mathrm{~cm}$ was considered. The PUL resistance, inductance, conductance and capacitance matrices were frequencydependent and referred to as Line 2 in [85].

Using steps similar to those in Example 1, the frequency-dependent PUL impedance (and admittance) was first approximated with a second-order positive-real rational functions. The accuracy of this approximation is demonstrated in Figure 6.13. The resulting rational functions were then synthesized with two sets of PUL impedance and admittance networks using two different approaches, namely lumped element based and Laplace element based. One series lumped model was constructed from each set of PUL networks for the transmission line and embedded in the circuit shown in Figure 6.14 for transient analysis. Figure 6.15 shows the comparison of transient responses between these two simulations, as well as the responses from IFFT. As evident, all the responses matched each other very well. 


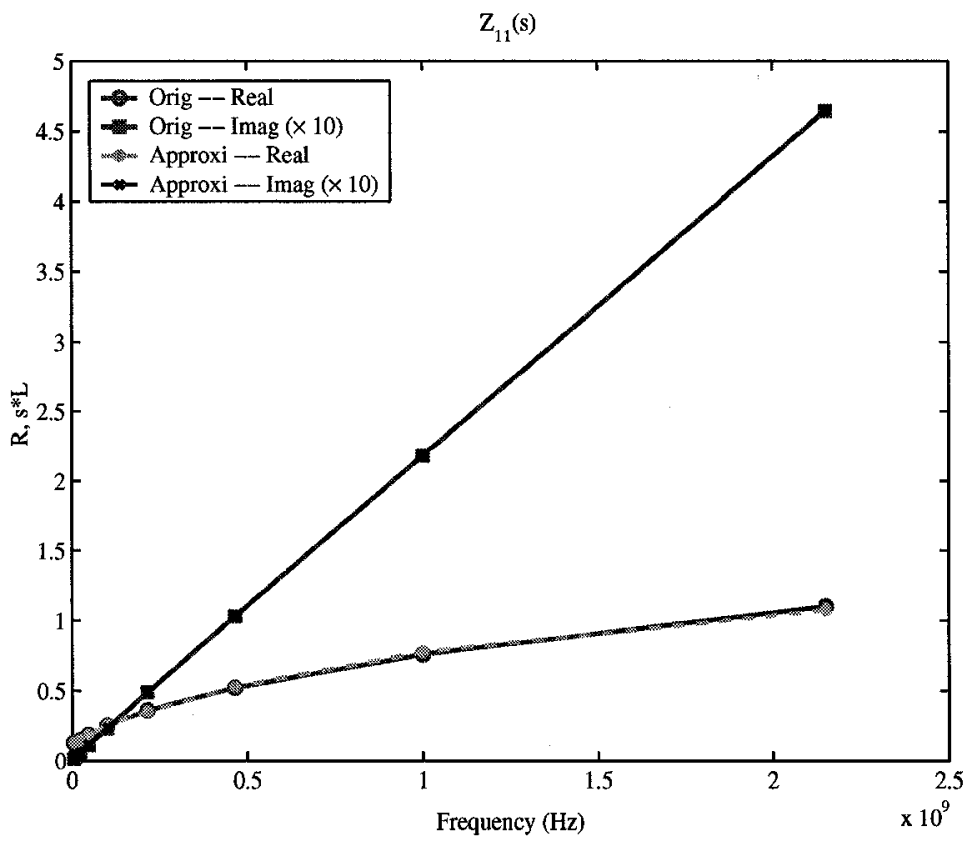

(a) $\mathcal{Z}_{1,1}$ vs. $\tilde{\mathcal{Z}}_{1,1}$.

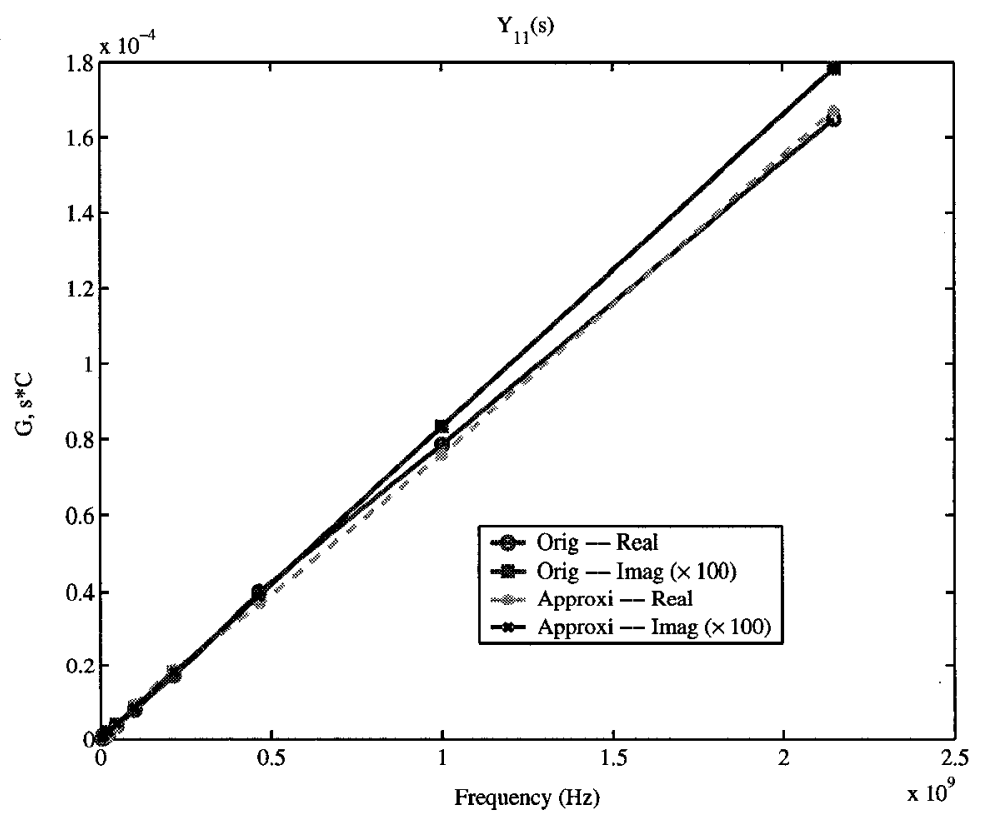

(b) $\mathcal{Y}_{1,1}$ vs. $\tilde{\mathcal{Y}}_{1,1}$.

Figure 6.11: Approximation of the PUL impedance and admittance. 


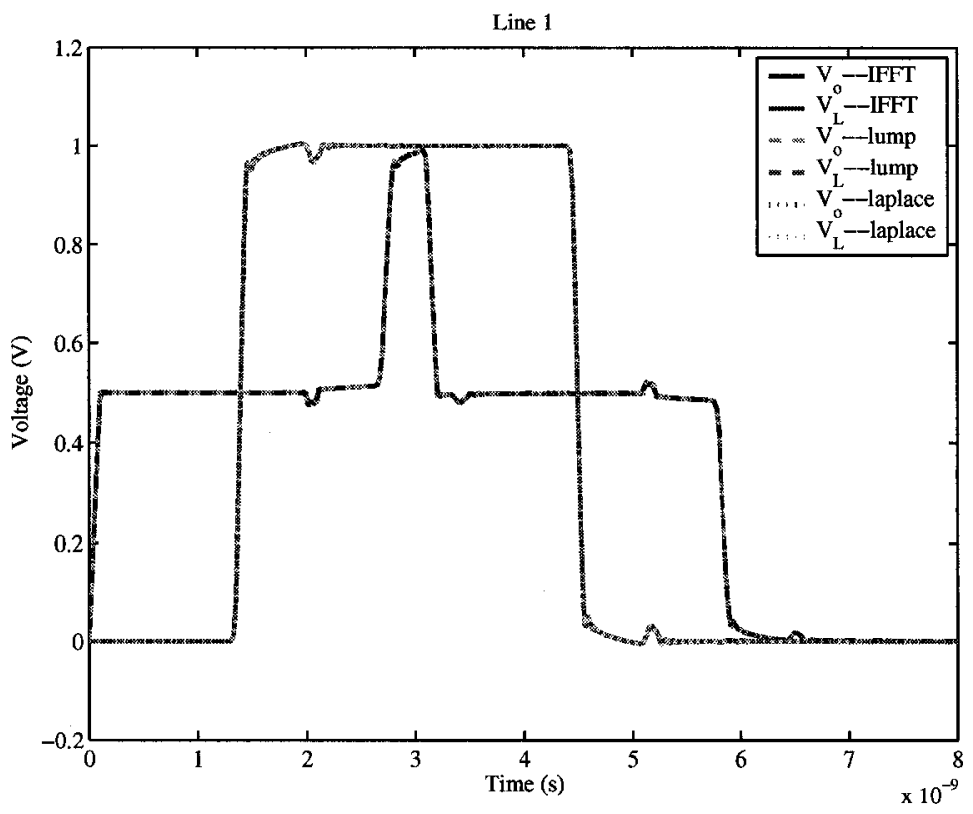

(a) Active line.

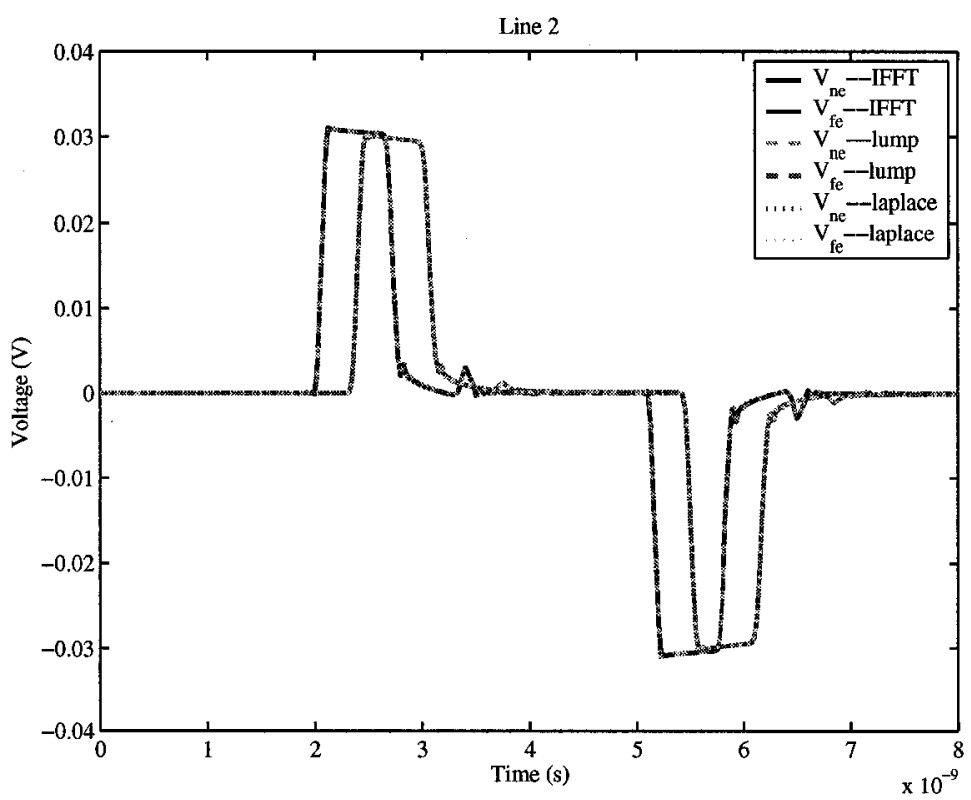

(b) Victim line.

Figure 6.12: Transient responses for Example 1. 


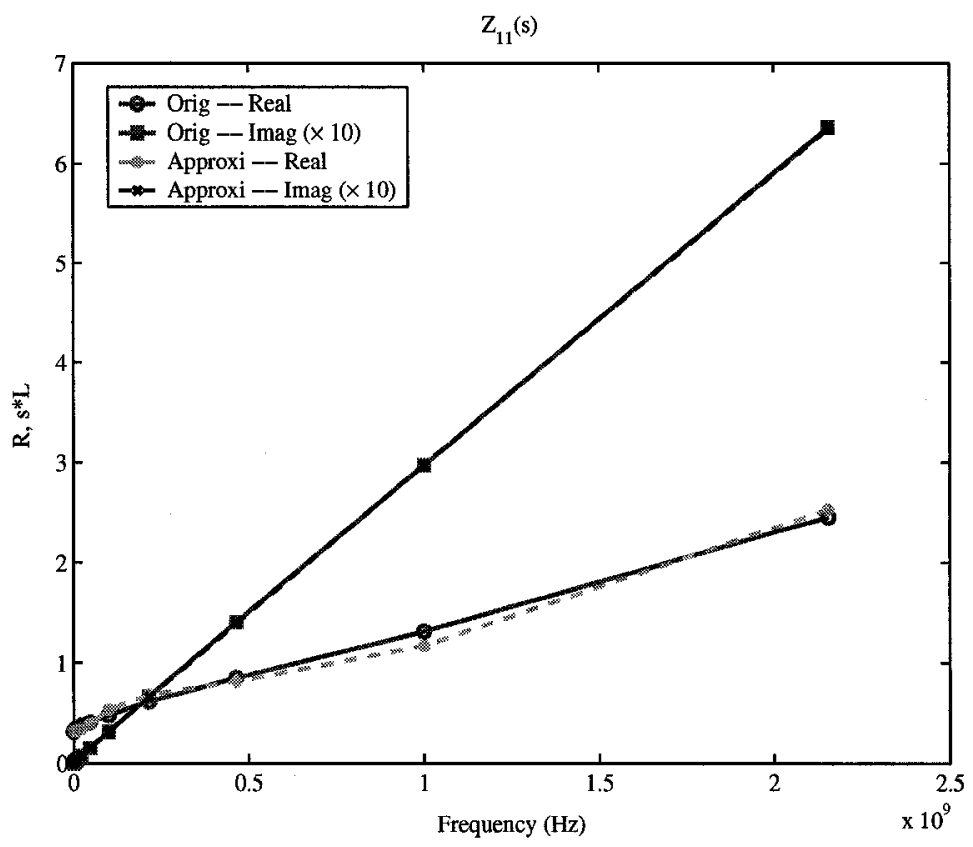

(a) $\mathcal{Z}_{1,1}$ vs. $\tilde{\mathcal{Z}}_{1,1}$.

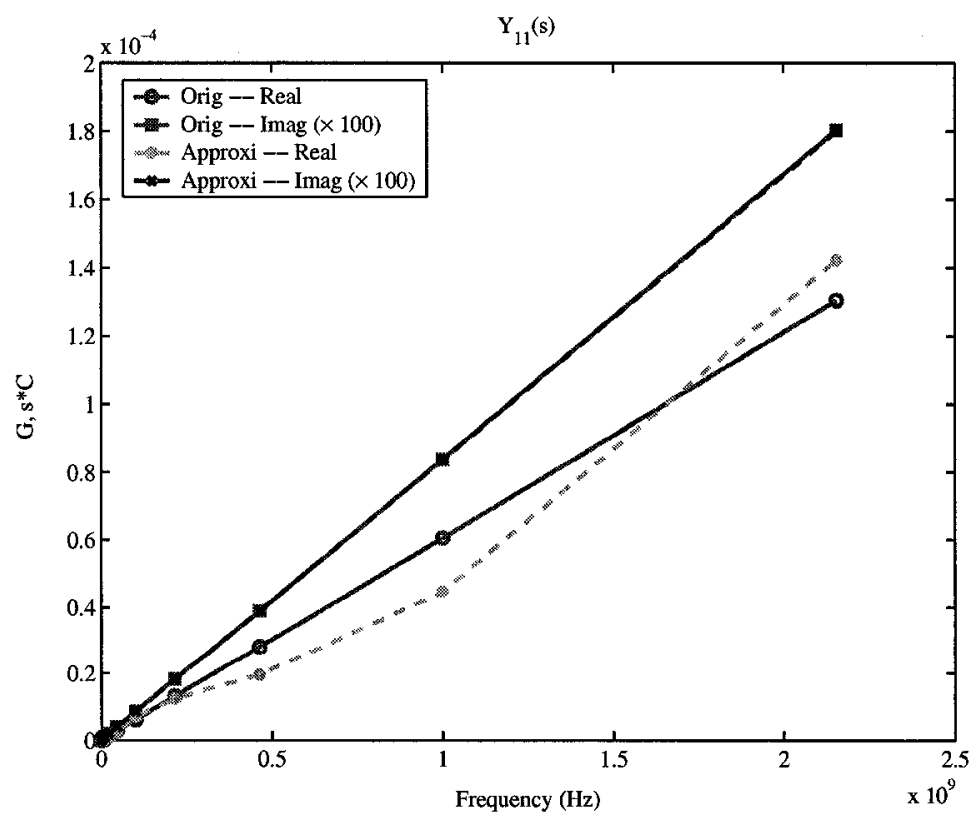

(b) $\mathcal{Y}_{1,1}$ vs. $\tilde{\mathcal{Y}}_{1,1}$

Figure 6.13: Approximation of the PUL impedance and admittance. 


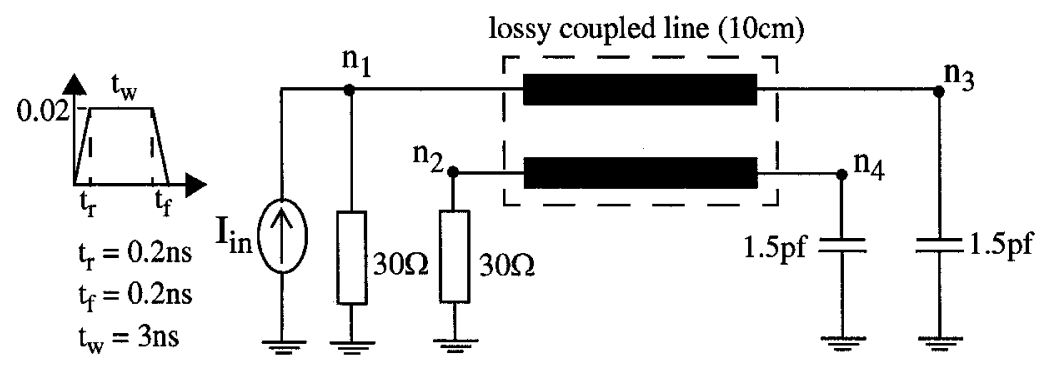

Figure 6.14: Circuit topology for Example 2.

\subsubsection{Example 3}

In this example, we investigated the performance of lumped and Laplace based network synthesis approaches as the number of conductors in the TL is increased significantly. For this purpose, the PUL parameters were extracted from a 25-conductor transmission line given by IBM [112]. Specifically, an $(m+1)$-conductor transmission line in this example denoted a TL composed of the first $m$ signal conductors and the ground conductor out of the 25 conductors. This in turn means that for a $(m+1)$-conductor transmission line, we considered the top-left $m \times m$ block from each of the four $24 \times 24$ matrices as its PUL parameters. The PUL capacitance and conductance for this TL were frequency-independent, and therefore no approximation was required for the PUL admittance matrix. On the other hand, the PUL impedance was approximated with positive-real rational functions, which were synthesized using both lumped element based and Laplace element based networks.

Similar to the above two examples, the constructed PUL networks were embedded into a circuit network for transient simulation. For the purpose of fair comparison, thirdorder rational approximation was applied to all cases and each $(m+1)$-conductor TL was embedded in a similar circuit network as depicted in Figure 6.16. All conductors were terminated with a $1 p F$ capacitor at the far end and a $50 \Omega$ resistor at the near end. All odd-numbered conductors were excited with an identical current source as shown in Figure 6.16. We conducted the transient simulation for $2,3, \ldots, 25$-conductor networks and 


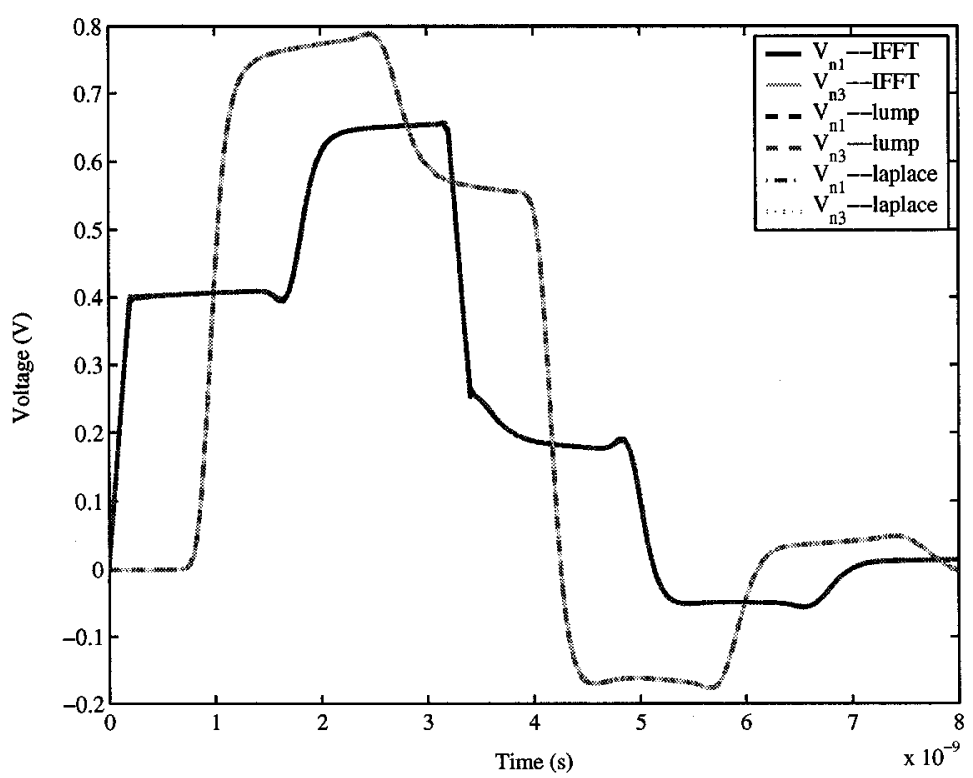

(a) Active line.

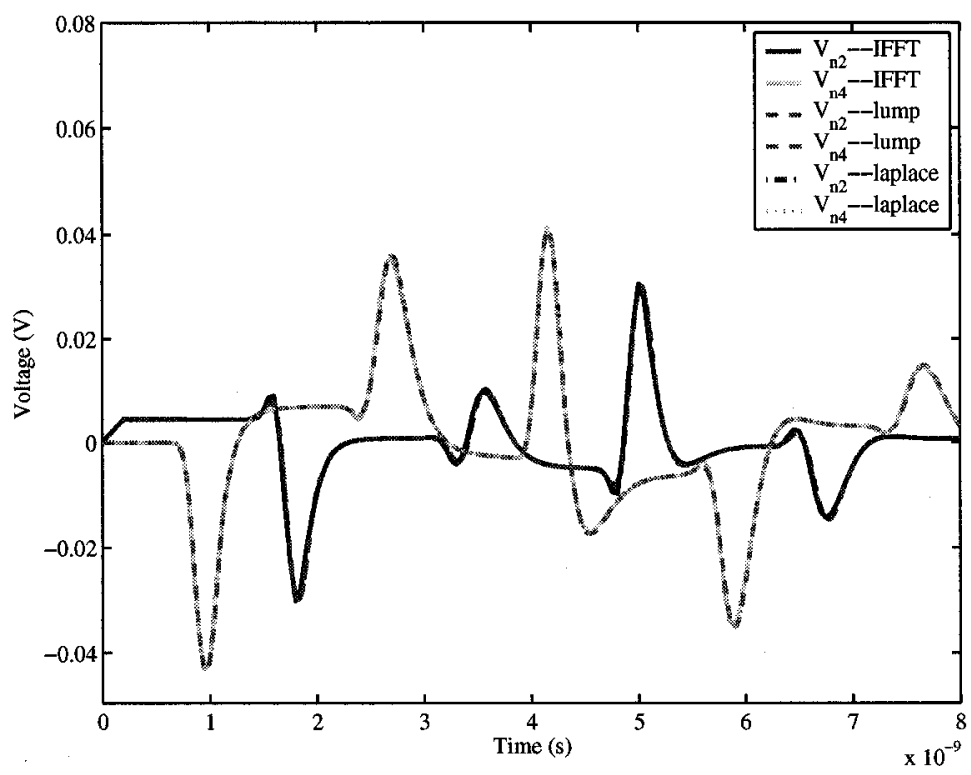

(b) Victim line.

Figure 6.15: Transient responses for Example 2. 


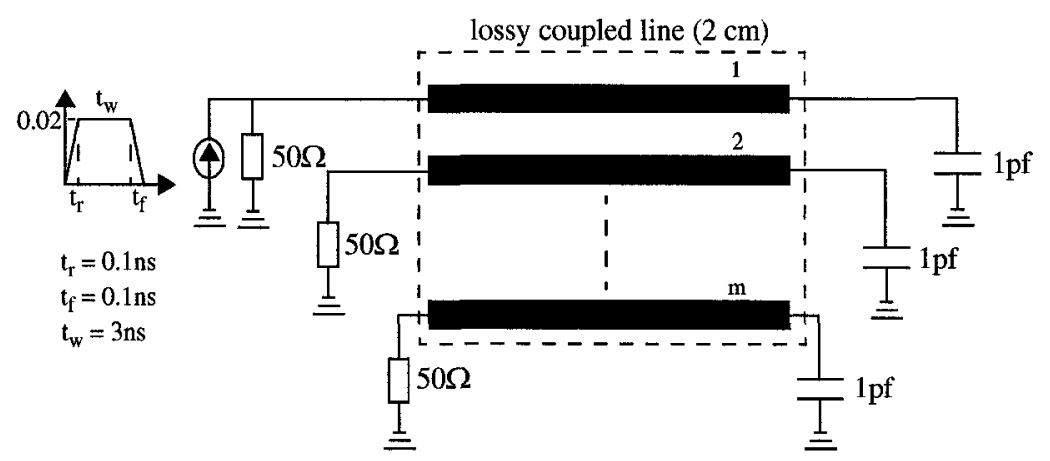

Figure 6.16: Circuit topology for Example 3.

each network was implemented twice using the lumped element based and Laplace element based PUL networks, respectively. Figures 6.17 and 6.18 show the transient responses at conductor 1 and 4 for the case of 6-conductor TL. The plots showed that both simulations produced very close responses compared to IFFT.

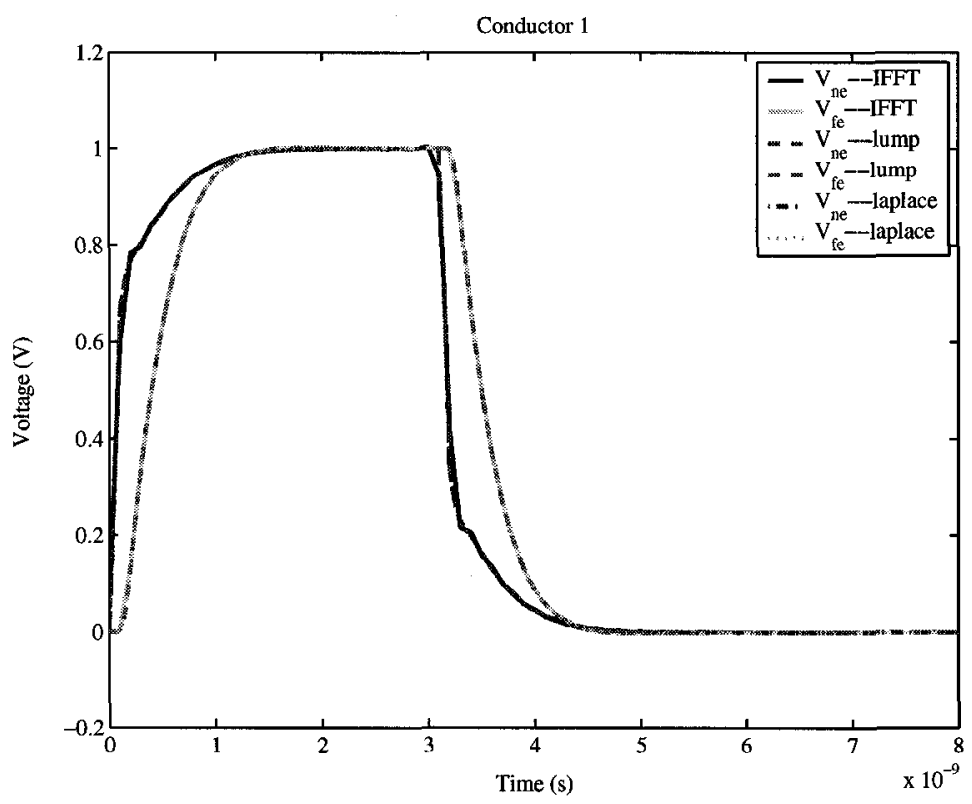

Figure 6.17: Transient responses at conductor 1 for the 6-conductor network.

When the number of conductors increased, we found that the simulation based on Laplace elements ran well for all cases. As an example, Figure 6.19 shows the transient response at conductor 15 from the 25 -conductor network simulation. On the other hand, 


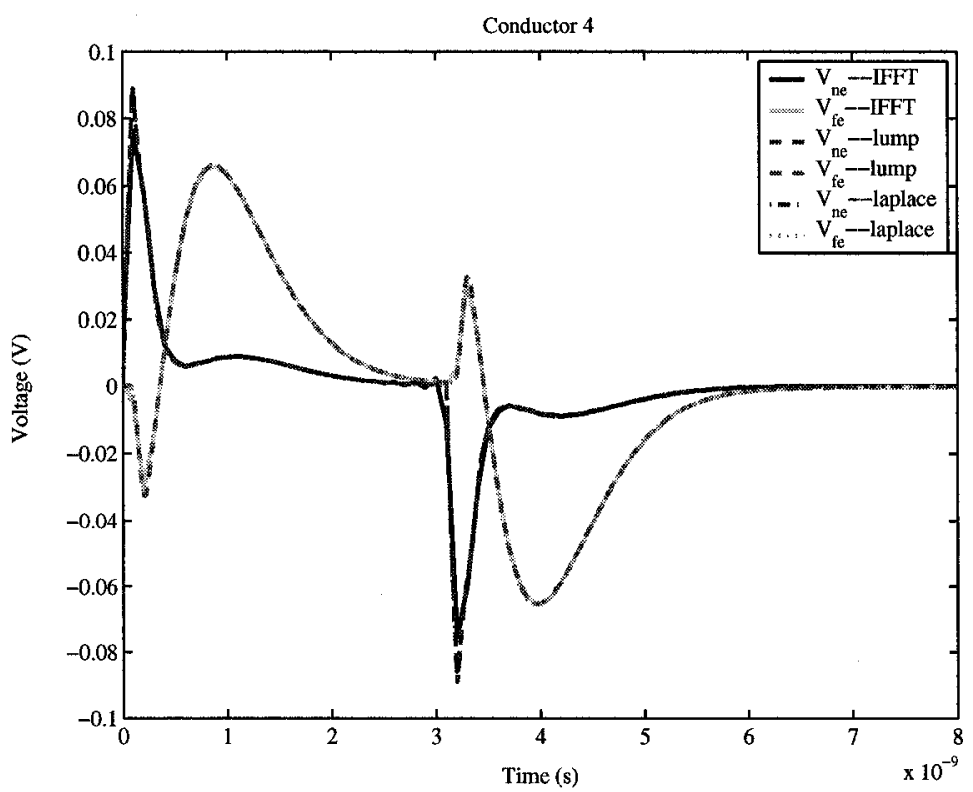

Figure 6.18: Transient responses at conductor 4 for the 6-conductor network.

the simulations based on the lumped PUL networks started to fail at the 11-conductor networks due to the convergence problem in HSPICE ${ }^{\circledR}$. A close investigation revealed some negative elements in the PUL impedance network. Note that this does not imply wrong implementation. The reason for this is that mutual impedance is not restricted to be PR after rational approximation even though the whole impedance matrix is PR. Some warnings were produced from HSPICE ${ }^{\circledR}$ in the presence of these negative elements, which contributed to the convergence problem.

Table 6.1 summarizes the CPU time needed to run the above examples. The CPU time reported in this table is based on simulation carried out on the Unix version of HSPICE ${ }^{\circledR}$. The table clearly indicates that the PUL network based on Laplace elements is generally more robust and efficient than the one based on lumped elements. 


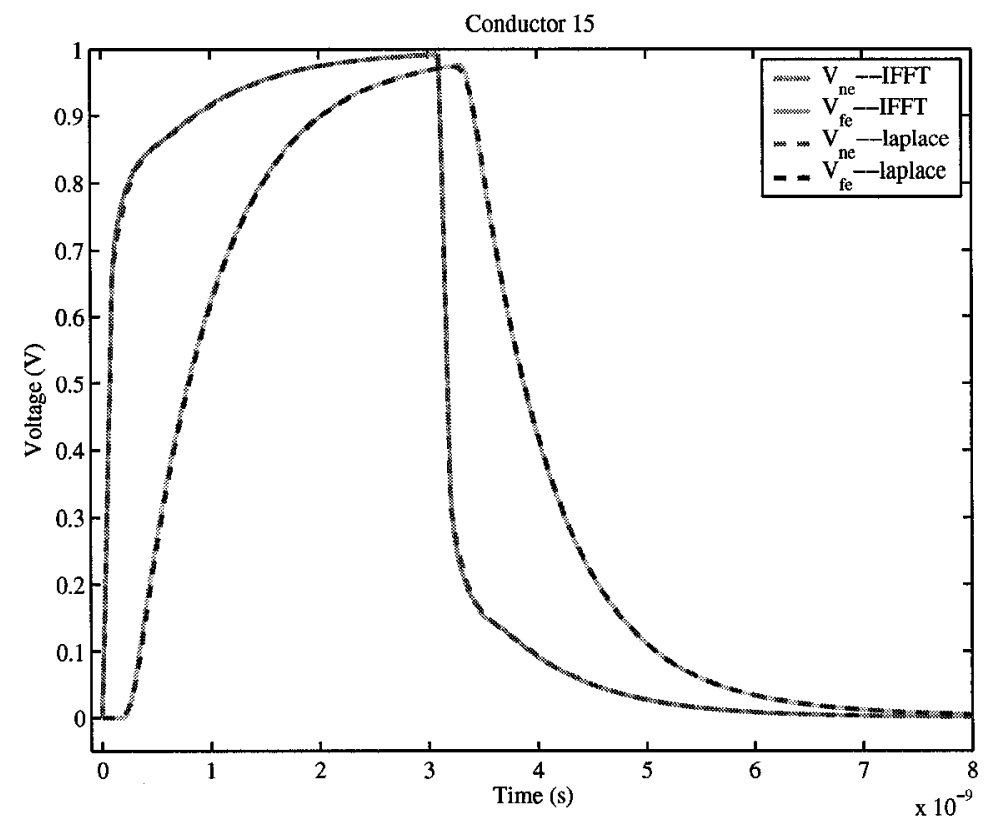

Figure 6.19: Transient responses at conductor 15 for the 25-conductor network.

Table 6.1: Transient simulation CPU time (seconds).

\begin{tabular}{|c|c|c|c|}
\hline Examples & $\begin{array}{c}\text { number of } \\
\text { conductors }\end{array}$ & $\begin{array}{c}\text { CPU Time } \\
\text { lumped model }\end{array}$ & $\begin{array}{c}\text { CPU Time } \\
\text { Laplace model }\end{array}$ \\
\hline \hline Example 1 & 3 & 16.1 & 1.97 \\
\hline Example 2 & 3 & 1.82 & 0.39 \\
\hline Example 3 & 3 & 1.62 & 0.32 \\
& 6 & 22.75 & 4.61 \\
& 9 & 60.25 & 17.00 \\
& 10 & 79.92 & 22.90 \\
& 11 & $\mathrm{x}$ & 29.22 \\
& 16 & $\mathrm{x}$ & 90.74 \\
& 25 & $\mathrm{x}$ & 295.79 \\
\hline
\end{tabular}




\section{Chapter 7}

\section{Analysis of Frequency-Dependent Interconnects Using Integrated Congruence Transform}

\subsection{Introduction}

The integrated congruence transform technique has been reviewed in Chapter 2 where it was applied to construct the reduced-order model for interconnects with frequency independent PUL parameters. ICT provides an efficient way in performing MOR for interconnect networks in that it does not require representing the interconnect with a large lumped circuit, which is an intermediate step for other MOR techniques, such as Arnoldi algorithm. However, ICT-MOR technique in its current form cannot be applied to interconnects with frequency-dependent parameters due to two immediate problems. The first problem is the need to employ the implicit-based technique for constructing the orthonormal basis in order to maintain the desirable numerical properties. The second problem is the passivity preservation [35].

To explain the reason why the implicit approach poses a problematic computational issue for the TLs with FD-PUL matrices, we rewrite (2.30)-(2.31) in the following general 
form,

$$
\begin{aligned}
& \hat{\mathcal{T}}_{0}\left(s_{o}\right) \boldsymbol{U}_{\mathrm{fd}}^{(0)}(x)=\mathbf{0} \\
& \hat{\mathcal{T}}_{0}\left(s_{o}\right) \boldsymbol{U}_{\mathrm{fd}}^{(i)}(x)=-\sum_{j=1}^{k} \hat{\mathcal{M}}_{j} \boldsymbol{U}_{\mathrm{fd}}^{(i-j)}(x)
\end{aligned}
$$

where $\hat{\mathcal{T}}_{0}\left(s_{o}\right)=\left(\boldsymbol{T} \frac{d}{d x}+\mathcal{N}_{0}+s_{o} \mathcal{M}_{0}\right) . \hat{\mathcal{M}}_{j}=\left(\mathcal{N}_{j}+s_{o} \mathcal{M}_{j}+\mathcal{M}_{j-1}\right) . \mathcal{N}_{j}$ and $\mathcal{M}_{j}$ are, respectively, the $j$-th moment of $\mathcal{N}(s)$ and $\mathcal{M}(s)$ computed at $s=s_{o}$ and the subscript "fd" was added to the moments to indicate that these moments belong to the frequencydependent case. It was proved by Theorem 1 in [21] that one of the crucial requirements that enables applying the implicit approach is basically to have the $i^{\text {th }}$ moment related only to the $(i-1)^{\text {th }}$ moment by a relation similar to (2.31). However, (7.2) demonstrates that such a relationship does not hold in the case of TLs with FD-PUL matrices. Hence, the implicit approach cannot be applied directly.

Passivity preservation is a key requirement that guarantees the stability of the subsequent SI simulation. It has been shown in [20] that the reduced system from ICT in (2.27) is passive. That proof, however, was derived for the case of frequency-independent PUL parameters and, further, relied on the fact that $\mathcal{N}$ and $\mathcal{M}$ are real matrices with certain properties. This is, however, no longer the case for the TLs with FD-PUL parameters. Therefore, it is not obvious in this latter case, how or whether an ICT-based algorithm is still capable of preserving the passivity.

This thesis addresses the above two problems by proposing a new technique that extends the idea of ICT so that it can handle the high-speed interconnects with FD-PUL matrices. This is carried out first through modeling the FD-PUL matrices with positivereal rational functions using the approach described in Chapter 6. Then including the PR rational functions into TEs leads to a generalized form of TEs which is given by a mixed set of linear differential algebraic equations. This is to be contrasted with the classical TEs 
describing the TL which are made up of pure differential equations. Two methodologies are proposed to construct the new formulation of TEs. One is based on synthesizing the PR rational functions with lumped network as discussed in Chapter 6. The other one is based on state-space representation for the rational functions. To maintain the numerical robustness of the ICT methodology, the idea of implicit moment matching technique has to be adapted for situations where the system is described by a set of mixed DAEs rather than pure differential equations. The resulting reduced-order model can be put in a state-space form of ordinary differential equations, and therefore passive balancing transformation, e.g. as in [19], can be applied to obtain further reduction in size if desired.

The rest of the chapter is organized as follows [35]. Section 7.2 describes the theoretical basis behind the proposed technique. The formulation for the generalized TEs is presented based on the first methodology, i.e., lumped network realization for the FD-PUL parameters. The alternative formulation based on the state-space representation will be discussed in Appendix E. Section 7.3 describes the numerical implementation of the proposed technique. Section 7.4 presents the numerical experiments to validate the accuracy of the proposed technique.

\subsection{Development of the proposed algorithm}

The proposed algorithm can be described in a two-stage process. The first stage, which is presented in Section 7.2.1, formulates the classical TEs, whose coefficients are frequencydependent, with general mixed set of DAEs, whose coefficients become frequency independent. This developed formulation is the key idea that enables the two main objectives of the proposed algorithm, namely applying the implicit approach in constructing the basis and guaranteeing the passivity of the reduced system. The second stage, given in Section 7.2.2, builds on this formulation to construct the reduced-order system. Section 7.2.3 presents the 
proof of passivity in the reduced-order system.

\subsubsection{Formulation of differential algebraic equations}

In order to simplify presenting the basic idea in the proposed formulation, we consider the special case of a TL with two conductors. Generalization of this idea to MTLs is straightforward. In this special case, the FD-PUL parameters become scalar functions of $s$. Since the PUL parameters are typically obtained in the form of tabulated data from measurements, empirical formulas, or electromagnetic simulation, computing the high order derivatives of these parameters (e.g., as it may be required in [21]) may not be feasible. To overcome this problem, it will be necessary to represent the variation of these parameters using some appropriate modeling techniques. Due to its robustness, the modeling approach introduced in Chapter 6 is employed. Specifically, the FD-PUL matrices are first approximated with certain PR rational functions, which are then synthesized with lumped networks. Using the resulting network representation, and the MNA formulation approach, one can put $\tilde{\mathcal{Z}}(s)$ in the following matrix form:

$$
\tilde{\mathcal{Z}}(s)=\boldsymbol{b}_{Z}^{T}\left(\mathcal{G}_{Z}+s \mathcal{C}_{Z}\right)^{-1} \boldsymbol{b}_{Z}
$$

where $b_{Z}$ is a vector that contains " 1 " in its first component and " 0 " otherwise. $\mathcal{G}_{Z}$ and $\mathcal{C}_{Z}$ are matrices composed of "stamps" contributed by the passive components. In general, these matrices have the following structures

$$
\mathcal{G}_{Z}=\left[\begin{array}{cc}
\mathcal{N}_{Z} & \mathcal{E}_{Z} \\
-\mathcal{E}_{Z}^{T} & \mathbf{0}
\end{array}\right], \quad \mathcal{C}_{Z}=\left[\begin{array}{cc}
\mathcal{Q}_{Z} & \mathbf{0} \\
\mathbf{0} & \mathcal{P}_{Z}
\end{array}\right]
$$

where $\mathcal{N}_{Z}, \mathcal{Q}_{Z}$, and $\mathcal{P}_{Z}$ are symmetric non-negative definite matrices that contain, in respective order, the stamps of resistive, capacitive and inductive elements. $\mathcal{E}_{Z}$ serves as a mapping operator with elements $\mathcal{E}_{z, i j} \in\{ \pm 1,0\}$. 
An analogous argument is made to show that $\tilde{\mathcal{Y}}(s)$ can be put in a similar matrix form,

$$
\tilde{\mathcal{Y}}(s)=\boldsymbol{b}_{Y}^{T}\left(\mathcal{G}_{Y}+s \mathcal{C}_{Y}\right)^{-1} \boldsymbol{b}_{Y}
$$

where the matrices $\mathcal{G}_{Y}$ and $C_{Y}$ have the same structure as that of $\mathcal{G}_{Z}$ and $\mathcal{C}_{Z}$, and $b_{Y}$ is a vector with a single unity entry and "0" otherwise entries. It should be noted here that, from the above construction, $\mathcal{C}_{Z}$ and $\mathcal{G}_{Z}+\mathcal{G}_{Z}^{T}$ (or $\mathcal{C}_{Y}$ and $\mathcal{G}_{Y}+\mathcal{G}_{Y}^{T}$ ) are non-negative definite.

The rationale used to represent both $\tilde{\mathcal{Z}}(s)$ and $\tilde{\mathcal{Y}}(s)$ for two-conductor TLs can be generalized and carried to the case of multi-conductor TLs. In that case, $\boldsymbol{b}_{Z}$ and $\boldsymbol{b}_{Y}$ will become rectangular matrices having a number of columns equal the number of conductors in the TL, with a single identity entry in each column and " 0 " entry otherwise. $\tilde{\mathcal{Z}}(s)$ and $\tilde{\mathcal{Y}}(s)$ become matrix-valued functions. They will be replaced with $\tilde{\mathcal{Z}}(s)$ and $\tilde{\mathcal{Y}}(s)$, respectively, in the later text to denote a general MTL case.

Assuming that the dimensions of the corresponding $\mathcal{G}_{Z}$ (or $\mathcal{C}_{Z}$ ) and $\mathcal{G}_{Y}\left(\right.$ or $\mathcal{C}_{Y}$ ) are given, respectively, by $n_{Z}$ and $n_{Y}$, we then proceed to show how to incorporate these approximate network functions into the TEs. This can be done by introducing the set of auxiliary variables $\boldsymbol{V}_{Z}(x, s)$ and $\boldsymbol{I}_{Y}(x, s)$ which are defined as follows,

$$
\begin{aligned}
\left(\mathcal{G}_{Z}+s \mathcal{C}_{Z}\right) \boldsymbol{V}_{Z}(x, s) & =\boldsymbol{b}_{Z} \boldsymbol{I}(x, s) \\
\left(\mathcal{G}_{Y}+s \mathcal{C}_{Y}\right) \boldsymbol{I}_{Y}(x, s) & =\boldsymbol{b}_{Y} \boldsymbol{V}(x, s)
\end{aligned}
$$

where $\boldsymbol{I}(x, s)$ and $\boldsymbol{V}(x, s)$ are, respectively, the currents and voltages at any point $x$ on the line, as introduced in (2.1). Substituting in the TEs in (2.1) for $\boldsymbol{R}(s)+s \boldsymbol{L}(s)$ and $\boldsymbol{G}(s)+s \boldsymbol{C}(s)$ using the approximate $\tilde{\mathcal{Z}}(s)$ and $\tilde{\mathcal{Y}}(s)$ given respectively by (7.3) and (7.5) 
yields,

$$
\tilde{\boldsymbol{T}} \frac{\partial}{\partial x} \tilde{\boldsymbol{X}}(x, s)=-(\tilde{\mathcal{N}}+s \tilde{\mathcal{M}}) \tilde{\boldsymbol{X}}(x, s)
$$

where,

$$
\begin{gathered}
\tilde{\boldsymbol{T}}=\left[\begin{array}{cccc}
\mathbf{0} & \boldsymbol{I}_{m} & \mathbf{0} & \mathbf{0} \\
\boldsymbol{I}_{m} & \mathbf{0} & \mathbf{0} & \mathbf{0} \\
\mathbf{0} & \mathbf{0} & \mathbf{0} & \mathbf{0} \\
\mathbf{0} & \mathbf{0} & \mathbf{0} & \mathbf{0}
\end{array}\right], \tilde{\mathcal{M}}=\left[\begin{array}{cccc}
\mathbf{0} & \mathbf{0} & \mathbf{0} & \mathbf{0} \\
\mathbf{0} & \mathbf{0} & \mathbf{0} & \mathbf{0} \\
\mathbf{0} & \mathbf{0} & \mathcal{C}_{Z} & \mathbf{0} \\
\mathbf{0} & \mathbf{0} & \mathbf{0} & \mathcal{C}_{Y}
\end{array}\right], \\
\tilde{\mathcal{N}}=\left[\begin{array}{cccc}
\mathbf{0} & \mathbf{0} & \boldsymbol{b}_{Z}^{T} & \mathbf{0} \\
\mathbf{0} & \mathbf{0} & \mathbf{0} & \boldsymbol{b}_{Y}^{T} \\
-\boldsymbol{b}_{Z} & \mathbf{0} & \mathcal{G}_{Z} & \mathbf{0} \\
\mathbf{0} & -\boldsymbol{b}_{Y} & \mathbf{0} & \mathcal{G}_{Y}
\end{array}\right], \tilde{\boldsymbol{X}}(x, s)=\left[\begin{array}{c}
\boldsymbol{I}(x, s) \\
\boldsymbol{V}(x, s) \\
\boldsymbol{V}_{Z}(x, s) \\
\boldsymbol{I}_{Y}(x, s)
\end{array}\right]
\end{gathered}
$$

It is important to highlight that the new equations relating the currents and voltages in (7.8) form a mixed set of linear DAEs. This represents a significant departure from the classical TEs which are pure differential equations. The new formulation requires adapting the basic construction algorithm presented in Section 2.7 for the frequency independent case to obtain the reduced-order system. The details of this adaptation are presented in the following subsection, Section 7.2.2.

\subsubsection{Construction of the reduced-order model}

Constructing the reduced-order model can be summed up in two main steps as follows.

\section{Step 1. Computation of the orthonormal basis $\mathcal{Q}(x)$}

The objective in this step is to employ the implicit approach to construct the orthonormal basis used in computing the reduced system matrices. For that purpose, we consider the 
DAEs in (7.8) and define $\tilde{\mathcal{X}}(x, s) \in \mathbb{C}^{\lambda \times 2 m}$, where $\lambda=2 m+n_{Z}+n_{Y}$, as the matrixvalued solution obtained under a set of $2 m$ BCs that have been mentioned earlier. The solution thus obtained may be expressed in the Taylor series expansion, i.e., $\tilde{\boldsymbol{X}}(x, s)=$ $\sum_{i=0}^{\infty} \tilde{U}^{(i)}(x)\left(s-s_{o}\right)^{i}$, where $\tilde{U}^{(i)}(x)$ are matrices of size $\lambda \times 2 m$ that are functions of $x$. Substituting this expansion in (7.8), and equating similar powers of $s$ will show that $\tilde{U}^{(i)}(x)$ are related recursively by relations similar to (2.30) and (2.31) with $\mathcal{T}\left(s_{o}\right), \mathcal{N}$ and $\mathcal{M}$ being replaced by $\tilde{\mathcal{T}}\left(s_{o}\right), \tilde{\mathcal{N}}$ and $\tilde{\mathcal{M}}$, respectively, where $\tilde{\mathcal{T}}\left(s_{o}\right)=\tilde{\boldsymbol{T}} \frac{d}{d x}+\tilde{\mathcal{N}}+s_{o} \tilde{\mathcal{M}}$. This observation provides the key idea that enables using the implicit approach in constructing an orthonormal basis for $\tilde{U}^{(i)}(x)$. Figure 7.1 presents a pseudocode representation for this approach. In the figure, we denote the desired basis by $\mathcal{Q}(x)$ and assume that it has the elements $\left\{\boldsymbol{v}^{(0)}(x), \boldsymbol{v}^{(1)}(x), \cdots\right\}$. The $\langle\cdot \mid \cdot\rangle$ and $\|\cdot\|$ define the inner product and norm mapping on $\mathcal{L}(0, d)$ and are given by

$$
\langle\boldsymbol{u}(x) \mid \boldsymbol{v}(x)\rangle=\int_{a}^{b} \boldsymbol{u}(x)^{T} \boldsymbol{v}(x) w(x) d x
$$

while $\|\boldsymbol{u}(x)\|=\langle\boldsymbol{u}(x) \mid \boldsymbol{u}(x)\rangle$. The appropriate BCs, expansion point and error criteria, as well as the particular choice of the parameters $a, b$, and $w(x)$ are further specific implementation issues that will be discussed in Section 7.3.

\section{Step 2. Extraction of the reduced-order model}

Given that the orthonormal basis $\mathcal{Q}(x)$ has been obtained in the previous process, we divide $\mathcal{Q}(x)$ into four portions as follows,

$$
\mathcal{Q}(x)=\left[\begin{array}{l}
\mathcal{Q}_{i}(x) \\
\mathcal{Q}_{v}(x) \\
\mathcal{Q}_{Z}(x) \\
\mathcal{Q}_{Y}(x)
\end{array}\right]
$$




\section{Algorithm 7.2.1: ICT-FD-PUL $\left(\tilde{\boldsymbol{T}}, \tilde{\mathcal{N}}, \tilde{\mathcal{M}}, s_{o}, e r r\right)$}

comment: $\operatorname{err}$ defines the user specified error criteria.

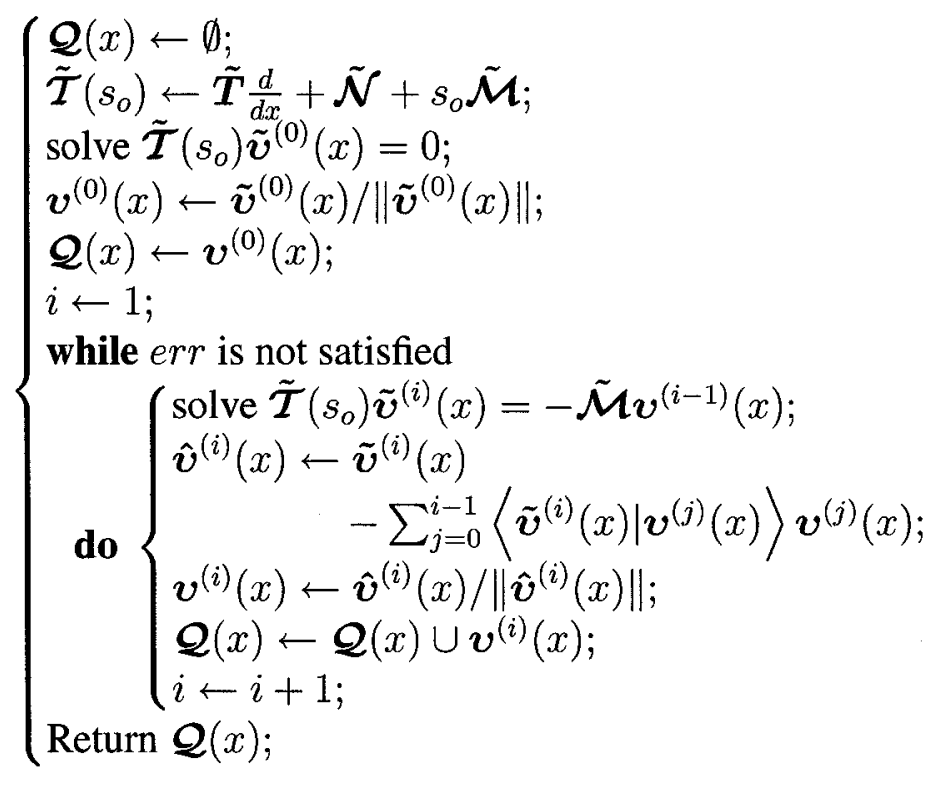

Figure 7.1: Pseudocode for computing the orthonormal basis $\mathcal{Q}(x)$.

where the first two portions $\mathcal{Q}_{i}(x)$ and $\mathcal{Q}_{v}(x)$ correspond to the current $\boldsymbol{I}(x, s)$ and voltage $\boldsymbol{V}(x, s)$ variables, respectively, and $\mathcal{Q}_{Z}(x)$ and $\mathcal{Q}_{Y}(x)$ correspond to the auxiliary variables $\boldsymbol{V}_{Z}(x, s)$ and $\boldsymbol{I}_{Y}(x, s)$, respectively. To proceed with constructing the reduced system, $\tilde{\boldsymbol{X}}(x, s)$ in $(7.8)$ is replaced by another set of variables through the following change of variables

$$
\tilde{\boldsymbol{X}}(x, s) \leftarrow \mathcal{Q}(x) \hat{\boldsymbol{X}}_{\mathcal{Q}}(s)
$$

where $\hat{\boldsymbol{X}}_{\mathcal{Q}}(s)$ is a vector containing a new set of auxiliary variables. (7.8) is then premultiplied by $\mathcal{Q}(x)^{T}$ and then integrated to yield,

$$
\left(\hat{\bar{T}}+\hat{\mathcal{\mathcal { N }}}_{1}+s \hat{\overline{\mathcal{M}}}\right) \hat{\boldsymbol{X}}_{\mathcal{Q}}(s)=0
$$


where $\hat{\overline{\mathcal{M}}}=\int_{0}^{d} \mathcal{Q}(x)^{T} \tilde{\mathcal{M}} \mathcal{Q}(x) d x, \hat{\overline{\mathcal{N}}}_{1}=\int_{0}^{d} \mathcal{Q}(x)^{T} \tilde{\mathcal{N}} \mathcal{Q}(x) d x$ and $\hat{\overline{\boldsymbol{T}}}=\int_{0}^{d} \mathcal{Q}(x)^{T} \tilde{\boldsymbol{T}} \frac{d \mathcal{Q}(x)}{d x} d x$.

The goal of the following steps is then to derive a relation between the terminal voltages $\boldsymbol{V}(0, s), \boldsymbol{V}(d, s)$ and currents $\boldsymbol{I}(0, s), \boldsymbol{I}(d, s)$. The voltages at the terminals of the TL are obtained from (7.12) and (7.13),

$$
\tilde{\boldsymbol{V}}(s)=\left[\begin{array}{l}
\mathcal{Q}_{v}(0) \\
\mathcal{Q}_{v}(d)
\end{array}\right] \hat{\boldsymbol{X}}_{\mathcal{Q}}(s)
$$

Defining the matrix $\mathcal{U}$ as

$$
\begin{aligned}
\mathcal{U} & =\int_{0}^{d} \frac{d\left(\mathcal{Q}_{i}^{T}(x) \mathcal{Q}_{v}(x)\right)}{d x} d x \\
& =\left(\mathcal{Q}_{i}(d)\right)^{T} \mathcal{Q}_{v}(d)-\left(\mathcal{Q}_{i}(0)\right)^{T} \mathcal{Q}_{v}(0)
\end{aligned}
$$

$\tilde{V}($ s) can then be obtained using

$$
-\mathcal{U} \hat{\boldsymbol{X}}_{\mathcal{Q}}(s)=\hat{\bar{b}} \tilde{\boldsymbol{V}}(s)
$$

where

$$
\hat{\bar{b}}=\left[\begin{array}{c}
\mathcal{Q}_{i}(0) \\
-\mathcal{Q}_{i}(d)
\end{array}\right]^{T}
$$

and the currents at the terminals of the TL can be described using $\mathcal{Q}_{i}(x)$

$$
\tilde{\boldsymbol{I}}(s)=\left[\begin{array}{c}
\mathcal{Q}_{i}(0) \\
-\mathcal{Q}_{i}(d)
\end{array}\right] \hat{\boldsymbol{X}}_{\mathcal{Q}}(s)=\hat{\overline{\boldsymbol{b}}}^{T} \hat{\boldsymbol{X}}_{\mathcal{Q}}(s)
$$

Let $\hat{\overline{\mathcal{N}}}_{2}=\hat{\overline{\boldsymbol{T}}}-\mathcal{U}$, and substitute in (7.14) using (7.17),

$$
(\underbrace{\hat{\mathcal{\mathcal { N }}}_{1}+\hat{\mathcal{\mathcal { N }}}_{2}}_{\hat{\mathcal{N}}}+s \hat{\overline{\mathcal{M}}}) \hat{\boldsymbol{X}}_{\mathcal{Q}}(s)=\hat{\bar{b}} \tilde{\boldsymbol{V}}(s)
$$


Hence the approximate constitutive relation between the terminal voltages and currents of the $\mathrm{TL}$ in the frequency-domain are obtained from

$$
\begin{aligned}
(\hat{\mathcal{\mathcal { N }}}+s \hat{\overline{\mathcal{M}}}) \hat{\boldsymbol{X}}_{\mathcal{Q}}(s) & =\hat{\bar{b}} \tilde{\boldsymbol{V}}(s) \\
\tilde{\boldsymbol{I}}(s) & =\hat{\overline{\boldsymbol{b}}}^{T} \hat{\boldsymbol{X}}_{\mathcal{Q}}(s)
\end{aligned}
$$

\subsubsection{Passivity preservation}

As mentioned earlier, one of the two main objectives of the proposed algorithm is to have the reduced-order system be passive by construction. The three conditions for passivity have been discussed in Section 2.2. Based on this discussion, it is enough to check the second and third conditions for the case of reduced system depicted in (7.21). Hence, the following theorem can be deduced.

Theorem 7.2.6 The reduced system defined by (7.21) and constructed using the basis $\mathcal{Q}(x)$ is passive if $\mathcal{Q}(x)$ is real.

Proof To prove that the reduced system is passive, we denote its admittance matrix by $\tilde{\boldsymbol{Y}}(s)$. From (7.21), we have,

$$
\tilde{\boldsymbol{Y}}(s)=\hat{\overline{\boldsymbol{b}}}^{T}(\hat{\mathcal{\mathcal { N }}}+s \hat{\mathcal{M}})^{-1} \hat{\bar{b}}
$$

From (7.22), it is straightforward to note that $\tilde{\boldsymbol{Y}}(s)$ satisfies the second condition of passivity. To prove that the third condition is satisfied as well, we note

$$
\begin{aligned}
& \tilde{\boldsymbol{Y}}(s)+\tilde{\boldsymbol{Y}}^{T}\left(s^{*}\right)=\hat{\overline{\boldsymbol{b}}}^{T}\left(\boldsymbol{F}^{*}(s)+\boldsymbol{F}(s)\right) \hat{\overline{\boldsymbol{b}}} \\
= & \hat{\overline{\boldsymbol{F}}}^{T}\left(s^{*}\right)\left(\hat{\mathcal{\mathcal { N }}}+s \hat{\overline{\mathcal{M}}}+\hat{\mathcal{\mathcal { N }}}^{T}+s^{*} \hat{\overline{\mathcal{M}}}^{T}\right) \hat{\boldsymbol{F}}(s)
\end{aligned}
$$

where $\boldsymbol{F}(s)=(\hat{\mathcal{\mathcal { N }}}+s \hat{\overline{\mathcal{M}}})^{-1}$, and $\hat{\overline{\boldsymbol{F}}}(s)=\boldsymbol{F}(s) \hat{\overline{\boldsymbol{b}}}$. The matrix on the left hand side of the 
equation is non-negative definite iff the following matrix is non-negative definite.

$$
(\hat{\mathcal{\mathcal { N }}}+s \hat{\overline{\mathcal{M}}})+\left(\hat{\mathcal{\mathcal { N }}}^{T}+s^{*} \hat{\mathcal{M}}^{T}\right)
$$

If we let $s=\sigma+\jmath \omega$, where $\sigma>0$, then the above matrix can be written as

$$
\begin{aligned}
& \left(\hat{\overline{\mathcal{N}}}_{1}+\hat{\mathcal{\mathcal { N }}}_{1}^{T}+\hat{\overline{\mathcal{N}}}_{2}+\hat{\overline{\mathcal{N}}}_{2}^{T}+\sigma\left(\hat{\overline{\mathcal{M}}}+\hat{\overline{\mathcal{M}}}^{T}\right)\right. \\
& \left.\quad+\jmath \omega\left(\hat{\overline{\mathcal{M}}}-\hat{\overline{\mathcal{M}}}^{T}\right)\right)=\left(\hat{\overline{\mathcal{N}}}_{1}+\hat{\overline{\mathcal{N}}}_{1}^{T}+2 \sigma \hat{\overline{\mathcal{M}}}\right)
\end{aligned}
$$

Two facts have been used to derive the above relation. This first one is that $\hat{\mathcal{N}}_{2}=-\hat{\mathcal{N}}_{2}^{T}$, as can be proved by the following manipulations,

$$
\begin{aligned}
\hat{\mathcal{\mathcal { N }}}_{2} & =\hat{\boldsymbol{T}}-\mathcal{U} \\
& =\int_{0}^{d}\left[\mathcal{Q}(x)^{T} \tilde{\boldsymbol{T}}\left(\frac{d \mathcal{Q}(x)}{d x}\right)-\frac{d\left(\boldsymbol{\mathcal { Q }}_{i}^{T}(x) \mathcal{Q}_{v}(x)\right)}{d x}\right] d x \\
& =\int_{0}^{d}\left[\mathcal{Q}_{v}(x)^{T}\left(\frac{d \boldsymbol{Q}_{i}(x)}{d x}\right)-\frac{d \boldsymbol{Q}_{i}(x)^{T}}{d x} \mathcal{Q}_{v}(x)\right] d x \\
& =-\hat{\hat{\mathcal{N}}}_{2}^{T}
\end{aligned}
$$

The other fact is that $\hat{\overline{\mathcal{M}}}$ is symmetric as can be seen from its definition,

$$
\begin{aligned}
\hat{\mathcal{M}} & =\int_{0}^{d} \mathcal{Q}(x)^{T} \tilde{\mathcal{M}} \mathcal{Q}(x) d x \\
& =\int_{0}^{d} \mathcal{Q}(x)^{T} \underbrace{\left[\begin{array}{ccccc}
\mathbf{0} & \mathbf{0} & \mathbf{0} & \mathbf{0} \\
\mathbf{0} & \mathbf{0} & \mathbf{0} & \mathbf{0} \\
\mathbf{0} & \mathbf{0} & \mathcal{C}_{Z} & \mathbf{0} \\
\mathbf{0} & \mathbf{0} & \mathbf{0} & \mathcal{C}_{Y}
\end{array}\right]}_{\text {symmetric non-negative definite }} \mathcal{Q}(x) d x
\end{aligned}
$$

The above analysis also shows that $\hat{\overline{\mathcal{M}}}$ is non-negative definite since it is given by the integration of congruence transformation of a non-negative definite matrix. Hence, to prove the theorem it remains only to prove that $\hat{\overline{\mathcal{N}}}_{1}+\hat{\overline{\mathcal{N}}}_{1}^{T}$ is non-negative definite. From definition, 
we have

$$
\begin{gathered}
\hat{\overline{\mathcal{N}}}_{1}+\hat{\overline{\mathcal{N}}}_{1}^{T}=\int_{0}^{d} \mathcal{Q}(x)^{T}\left(\tilde{\mathcal{N}}+\tilde{\mathcal{N}}^{T}\right) \mathcal{Q}(x) d x \\
=\int_{0}^{d} \mathcal{Q}(x)^{T}\left[\begin{array}{cccc}
0 & 0 & 0 & 0 \\
0 & 0 & 0 & 0 \\
0 & 0 & \mathcal{G}_{Z}+\mathcal{G}_{Z}^{T} & 0 \\
0 & 0 & 0 & \mathcal{G}_{Y}+\mathcal{G}_{Y}^{T}
\end{array}\right] \mathcal{Q}(x) d x
\end{gathered}
$$

Since the matrices $\mathcal{G}_{Z}+\mathcal{G}_{Z}^{T}$ and $\mathcal{G}_{Y}+\mathcal{G}_{Y}^{T}$ are non-negative definite by construction, it is easy to conclude that $\hat{\overline{\mathcal{N}}}_{1}+\hat{\overline{\mathcal{N}}}_{1}^{T}$ is non-negative definite and the reduced system is passive.

\subsection{Implementation issues}

In describing the development of the proposed algorithm in the previous section, it was shown that constructing the orthonormal basis $\mathcal{Q}(x)$ requires repeated solution of the DAEs system in ( $i i)$ in Algorithm 7.2.1. While solving (ii) is easy for the case of frequencyindependent parameters due to the availability of closed-form solution based on the matrix exponential approach [113], it becomes challenging for the system with frequencydependent parameters. This is due to having $\tilde{T}$ singular by definition. This fact leaves us with DAEs numerical solvers as the sole option for solving (ii).

This section outlines briefly adapting a procedure presented in [21] to handle this problem using Chebyshev polynomials [89]. In this technique, $\tilde{\boldsymbol{v}}^{(i)}(x)$ and $\boldsymbol{v}^{(i-1)}(x)$ are represented as summations of Chebyshev polynomials with coefficient matrices given by $\tilde{\Xi}_{i, h}$ and $\Xi_{i-1, h}$, respectively, with $h=0, \ldots, H$. Substituting from this Chebyshev series in (ii), and using various properties of the Chebyshev polynomials transforms the DAEs into the following set of algebraic equations [21],

$$
\left[\boldsymbol{A}_{D}+\frac{2}{d}\left(\boldsymbol{I}_{H+1} \otimes \tilde{\boldsymbol{T}}\right)\right] \tilde{\boldsymbol{\Phi}}_{i}=-\boldsymbol{A}_{M} \boldsymbol{\Phi}_{i-1}
$$


where

$$
\boldsymbol{A}_{D}=\left[\begin{array}{cccccc}
-\tilde{\boldsymbol{D}} & \frac{1}{4} \tilde{\boldsymbol{D}} & \frac{1}{3} \tilde{\boldsymbol{D}} & -\frac{1}{8} \tilde{\boldsymbol{D}} & \frac{1}{15} \tilde{\boldsymbol{D}} & \ldots \\
-\tilde{\boldsymbol{D}} & 0 & \frac{1}{2} \tilde{\boldsymbol{D}} & 0 & 0 & \ldots \\
0 & -\frac{1}{4} \tilde{\boldsymbol{D}} & 0 & \frac{1}{4} \tilde{\boldsymbol{D}} & 0 & \ldots \\
\vdots & \vdots & \ddots & \ddots & \ddots & \ddots \\
0 & 0 & 0 & 0 & 0 & \ldots \\
0 & 0 & 0 & 0 & 0 & \ldots \\
& \frac{(-1)^{H-2}}{(H-2)^{2}-1} \tilde{\boldsymbol{D}} & \frac{(-1)^{H-1}}{(H-1)^{2}-1} \tilde{\boldsymbol{D}} & \frac{(-1)^{H}}{H^{2}-1} \tilde{\boldsymbol{D}} \\
& 0 & & 0 & 0 \\
& 0 & & 0 & 0 \\
& -\frac{1}{2(H-1)} \tilde{\boldsymbol{D}} & 0 & \frac{1}{2(H-1)} \tilde{\boldsymbol{D}} \\
& 0 & -\frac{1}{2 H} \tilde{\boldsymbol{D}} & 0
\end{array}\right]
$$

$\boldsymbol{A}_{M}$ possesses the same structure as $\boldsymbol{A}_{D}$ except replacing $\tilde{\boldsymbol{D}}$ with $\tilde{\mathcal{M}} . \tilde{\boldsymbol{D}}=\tilde{\mathcal{N}}+s_{0} \tilde{\mathcal{M}}$, $\boldsymbol{\Phi}_{i-1}=\left[\boldsymbol{\Xi}_{i-1,0}{ }^{T} \ldots \boldsymbol{\Xi}_{i-1, H}{ }^{T}\right]^{T}, \tilde{\boldsymbol{\Phi}}_{i}=\left[\tilde{\boldsymbol{\Xi}}_{i, 0}{ }^{T} \ldots \tilde{\boldsymbol{\Xi}}_{i, H}{ }^{T}\right]^{T}$, and $\boldsymbol{I}_{H+1}$ is an $(H+1) \times(H+1)$ identity matrix.

The method presented here is essentially a Galerkin method and the particular choice of Chebyshev is motivated by the convenience that this family of orthogonal polynomials can provide means in solving the DAEs. More specifically, having the ability to represent the derivative of Chebyshev by a summation of Chebyshev makes it easy to derive the algebraic system in (7.24). Another advantage is that through choosing $a=-1, b=1$ and $w(x)=1 / \sqrt{1-x^{2}}$, the inner-product operation reduces to simple inner-product of the Chebyshev coefficients, i.e.,

$$
\langle\boldsymbol{u}(x) \mid \boldsymbol{v}(x)\rangle=\pi \boldsymbol{U}_{0}{ }^{T} \boldsymbol{V}_{0}+\frac{\pi}{2} \sum_{h=1}^{H} \boldsymbol{U}_{h}{ }^{T} \boldsymbol{V}_{h}
$$

where $\boldsymbol{U}_{h}$ and $\boldsymbol{V}_{h}, h=0, \ldots, H$, denote the Chebyshev coefficients of $\boldsymbol{u}(x)$ and $\boldsymbol{v}(x)$, respectively, when they are expanded using $H$ order Chebyshev polynomials, i.e., $u(x)=$ 
$\sum_{h=0}^{H} \boldsymbol{U}_{h} T_{h}(x), \boldsymbol{v}(x)=\sum_{h=0}^{H} \boldsymbol{V}_{h} T_{h}(x)$ and $T_{h}(x)$ represents the Chebyshev polynomials of the first kind.

Furthermore, numerical computations demonstrate that Chebyshev polynomials converge with few Chebyshev coefficients. Typically the number of coefficients, $H$, required to have an accurate solution is less then 20. It also should be stressed that the number of coefficients used to reach the solution has no impact on the size of the reduced system and therefore does not influence the time-domain simulation.

\subsubsection{Boundary conditions}

Another issue that arises in the course of generating the basis $\mathcal{Q}(x)$, is the computation of consistent initial conditions in solving $(i)$ and $(i i)$ for $\tilde{\boldsymbol{v}}^{(0)}(x)$ and $\tilde{\boldsymbol{v}}^{(i)}(x)$, respectively. For that purpose, $\tilde{\boldsymbol{v}}^{(i)}(x),(i \geq 0)$ is partitioned into four sets of variables as follows,

$$
\tilde{\boldsymbol{v}}^{(i)}(x)=\left[\begin{array}{c}
\tilde{\boldsymbol{v}}_{I}(x) \\
\tilde{\boldsymbol{v}}_{V}(x) \\
\tilde{\boldsymbol{v}}_{Z}(x) \\
\tilde{\boldsymbol{v}}_{Y}(x)
\end{array}\right]
$$

Recall that the objective is to generate an orthonormal basis for the derivatives of $\tilde{\boldsymbol{X}}(x, s)$, which represents the solution matrix of (7.8) using a $2 m \times 2 m$ identity matrix at the ports of the TL. It is therefore easy to see that this condition indicates that

$$
\begin{array}{ll}
{\left[\begin{array}{l}
\tilde{\boldsymbol{v}}_{V}^{(0)}(0) \\
\tilde{\boldsymbol{v}}_{V}^{(0)}(d)
\end{array}\right]=\left[\begin{array}{cc}
\boldsymbol{I}_{m} & \mathbf{0} \\
\mathbf{0} & \boldsymbol{I}_{m}
\end{array}\right]} \\
{\left[\begin{array}{l}
\tilde{\boldsymbol{v}}_{V}^{(i)}(0) \\
\tilde{\boldsymbol{v}}_{V}^{(i)}(d)
\end{array}\right]=\left[\begin{array}{ll}
\mathbf{0} & \mathbf{0} \\
\mathbf{0} & \mathbf{0}
\end{array}\right]} & i>0
\end{array}
$$

where $\boldsymbol{I}_{m}$ is an $m \times m$ identity matrix. The above conditions can then be used to derive the initial conditions for the rest of variables $\tilde{\boldsymbol{v}}_{I}^{(i)}(0), \tilde{\boldsymbol{v}}_{Z}^{(i)}(0)$ and $\tilde{\boldsymbol{v}}_{Y}^{(i)}(0),(i \geq 0)$. To 
this end, we use the notion of state transition matrix (STM) for systems described by linear differential equations. An STM is defined as the transformation matrix that maps the system from the state at $x=x_{1}$ to the state at $x=x_{2}$. If $\Psi\left(x_{1}, x_{2}\right)$ is the STM for the system in (i), then

$$
\tilde{\boldsymbol{v}}^{(0)}(d)=\boldsymbol{\Psi}(0, d) \tilde{\boldsymbol{v}}^{(0)}(0)
$$

and for the type of forced system in (ii), we have

$$
\tilde{\boldsymbol{v}}^{(i)}(d)=\boldsymbol{\Psi}(0, d)\left[\int_{0}^{d} \boldsymbol{\Psi}(x, 0) \mathfrak{F}^{(i-1)}(x) d x+\tilde{\boldsymbol{v}}^{(i)}(0)\right]
$$

where

$$
\mathfrak{F}^{(i-1)}(x)=-\tilde{\mathcal{M}} \boldsymbol{v}^{(i-1)}(x)
$$

Let $\boldsymbol{\Psi}(0, d)$ be partitioned into $4 \times 4$ block matrices, $\left[\boldsymbol{K}_{i j}\right]$. Then (7.29) can be written as follows,

$$
\left[\begin{array}{l}
\tilde{\boldsymbol{v}}_{I}^{(0)}(d) \\
\tilde{\boldsymbol{v}}_{V}^{(0)}(d) \\
\tilde{\boldsymbol{v}}_{Z}^{(0)}(d) \\
\tilde{\boldsymbol{v}}_{Y}^{(0)}(d)
\end{array}\right]=\left[\begin{array}{llll}
\boldsymbol{K}_{11} & \boldsymbol{K}_{12} & \boldsymbol{K}_{13} & \boldsymbol{K}_{14} \\
\boldsymbol{K}_{21} & \boldsymbol{K}_{22} & \boldsymbol{K}_{23} & \boldsymbol{K}_{24} \\
\boldsymbol{K}_{31} & \boldsymbol{K}_{32} & \boldsymbol{K}_{33} & \boldsymbol{K}_{34} \\
\boldsymbol{K}_{41} & \boldsymbol{K}_{42} & \boldsymbol{K}_{43} & \boldsymbol{K}_{44}
\end{array}\right]\left[\begin{array}{l}
\tilde{\boldsymbol{v}}_{I}^{(0)}(0) \\
\tilde{\boldsymbol{v}}_{V}^{(0)}(0) \\
\tilde{\boldsymbol{v}}_{Z}^{(0)}(0) \\
\tilde{\boldsymbol{v}}_{Y}^{(0)}(0)
\end{array}\right]
$$

Substituting from (7.27) into the second block row of (7.32) and using the definition of the auxiliary variables introduced in (7.6) and (7.7),

$$
\begin{aligned}
\tilde{\boldsymbol{v}}_{Z}^{(0)}(0) & =\left.\left(\mathcal{G}_{\boldsymbol{Z}}+s \mathcal{C}_{\boldsymbol{Z}}\right)^{-1} \boldsymbol{b}_{Z} \boldsymbol{I}(0, s)\right|_{s=s_{o}} \\
& =\left(\mathcal{G}_{\boldsymbol{Z}}+s_{o} \mathcal{C}_{\boldsymbol{Z}}\right)^{-1} \boldsymbol{b}_{Z} \tilde{\boldsymbol{v}}_{I}^{(0)}(0) \\
\tilde{\boldsymbol{v}}_{Y}^{(0)}(0) & =\left.\left(\mathcal{G}_{\boldsymbol{Y}}+s \mathcal{C}_{\boldsymbol{Y}}\right)^{-1} \boldsymbol{b}_{Y} \boldsymbol{V}(0, s)\right|_{s=s_{o}} \\
& =\left(\mathcal{G}_{\boldsymbol{Y}}+s_{o} \mathcal{C}_{\boldsymbol{Y}}\right)^{-1} \boldsymbol{b}_{Y} \tilde{\boldsymbol{v}}_{V}^{(0)}(0)
\end{aligned}
$$


The required initial condition for $\tilde{\boldsymbol{v}}_{I}^{(0)}(0)$ is obtained as follows,

$$
\begin{aligned}
\tilde{\boldsymbol{v}}_{I}^{(0)}(0)=- & \left(\boldsymbol{K}_{21}+\boldsymbol{K}_{23}\left(\mathcal{G}_{Z}+s_{o} \mathcal{C}_{Z}\right)^{-1} \boldsymbol{b}_{Z}\right)^{-1} \\
& {\left[\left(\boldsymbol{K}_{22}+\boldsymbol{K}_{24}\left(\mathcal{G}_{Y}+s_{o} \mathcal{C}_{Y}\right)^{-1} \boldsymbol{b}_{Y}\right) \tilde{\boldsymbol{v}}_{V}^{(0)}(0)-\tilde{\boldsymbol{v}}_{V}^{(0)}(d)\right] }
\end{aligned}
$$

Using the initial conditions derived above, it is possible to compute $\tilde{\boldsymbol{v}}^{(0)}(x)$ from the initial conditions and the STM form

$$
\tilde{\boldsymbol{v}}^{(0)}(x)=\mathbf{\Psi}(0, x) \tilde{\boldsymbol{v}}^{(0)}(0)
$$

In the actual implementation, we obtain $\tilde{\boldsymbol{v}}^{(0)}(x)$ as a Chebyshev series through solving a system of algebraic equations similar to that shown in (7.24), but with a different right-side matrix,

$$
\left[\boldsymbol{A}_{D}+\frac{2}{d}\left(\boldsymbol{I}_{H+1} \otimes \tilde{\boldsymbol{T}}\right)\right] \tilde{\boldsymbol{\Phi}}_{0}=\Omega_{0}
$$

where

$$
\boldsymbol{\Omega}_{0}=[\tilde{\boldsymbol{v}}^{(0)}(0)^{T} \underbrace{0 \cdots 0}_{H}]^{T} .
$$

The obtained solution vector, $\tilde{\Phi}_{0}$, is then partitioned into $H+1$ block matrices as, $\tilde{\Phi}_{0}=$ $\left[\tilde{\boldsymbol{\Xi}}_{0,0}{ }^{T} \cdots \tilde{\boldsymbol{\Xi}}_{0, H}^{T}\right]^{T}$ and $\tilde{\boldsymbol{v}}^{(0)}(x)$ is obtained as a Chebyshev series with $\tilde{\boldsymbol{\Xi}}_{0, h}$ as the Chebyshev coefficient matrices, i.e.,

$$
\tilde{\boldsymbol{v}}^{(0)}(x)=\sum_{h=0}^{H} \tilde{\Xi}_{0, h} T_{h}(2 x / d-1)
$$

Obtaining $\tilde{\boldsymbol{v}}^{(i)}(0),(i>0)$ is performed through using (7.30) and the boundary conditions (7.28) in a similar manner to computing $\tilde{\boldsymbol{v}}^{(0)}(0)$. Computing the $\operatorname{STM} \Psi(0, d)$ is carried out by solving (i) using an identity matrix as an initial condition. The solution matrix is then obtained by using the algebraic system in (7.37), and the obtained solution then represents 
the Chebyshev coefficients of $\Psi(0, x)$.

\subsubsection{Expansion point and error criteria}

The issue of selecting an appropriate expansion point $s_{o}$ has been addressed in [114] in the context of MOR for lumped networks. The heuristic guidelines developed in [114] is incorporated in the proposed algorithm, where $s_{o}$ is suggested to take a positive real value around the maximum frequency point of interest. In fact, numerical results demonstrate that choosing $s_{o}$ according to this rule produces reduced systems close to the optimal size, where it was noticed that a second level reduction based on passive balancing transformations does not lead to further significant reduction.

Another important implementation issue is the problem of defining error criteria to terminate the algorithm as shown in Figure 7.1. For that purpose we have adopted a simple procedure based on comparing the admittance matrix of the reduced system, $\tilde{\boldsymbol{Y}}(s)$, with the admittance matrix of the TL, $\boldsymbol{Y}(s)$, computed using the exponential stamp, as follows,

$$
\frac{|\boldsymbol{Y}(\mathcal{S})-\tilde{\boldsymbol{Y}}(\mathcal{S})|}{|\boldsymbol{Y}(\mathcal{S})|} \leq \epsilon_{\mathcal{S}}
$$

where $\epsilon_{\mathcal{S}}$ contains the user-specified maximum relative error at each frequency point specified in the frequency set of $\mathcal{S}$. It should be noted here that the checking for (7.38) is very fast since $\boldsymbol{Y}(\mathcal{S})$ can be obtained from stamp and only computed once, and $\tilde{\boldsymbol{Y}}(\mathcal{S})$ is evaluated for the reduced-order system. The above criteria are demonstrated to be efficient by several numerical examples.

\subsubsection{Complexity analysis}

In order to implement the proposed algorithm, we need to solve (7.37) once and (7.24) $q-1$ times for a $q$-order approximation. It can be observed that we just need to do lower/upper 
matrix (L/U) decomposition once on $\left[\boldsymbol{A}_{D}+\frac{2}{d}\left(\boldsymbol{I}_{H+1} \otimes \tilde{\boldsymbol{T}}\right)\right]$, and forward/backward (F/B) substitution $q$ times. This is similar to other MOR techniques, like PRIMA. However, the

size of the matrix $\left[\boldsymbol{A}_{D}+\frac{2}{d}\left(\boldsymbol{I}_{H+1} \otimes \tilde{\boldsymbol{T}}\right)\right]$ in this case is typically smaller. More specifically, if we denote the size of the matrix by $N$, then $N=H \times\left(2 m+n_{Z}+n_{Y}\right)$, where $H$ is the highest order of Chebyshev polynomials used to approximate the $x$-dependent moments. The order $H$ is typically less than 20. $m$ is the number of conductors of the TL. $n_{Z}$ and $n_{Y}$ represent the orders of matrices used to formulate the PUL impedance and admittance networks. $n_{Z}$ and $n_{Y}$ are usually small since the orders of the rational functions used to approximate the PUL impedance and admittance, are usually small $(\leq 4)$. An alternative implementation methodology of the proposed algorithm based on the state-space representation for the FD-PUL matrices will be discussed in Appendix E.

\subsection{Numerical examples}

The proposed algorithm was implemented and applied to several transmission line networks. The simulation results are presented in this section to validate the performance of the algorithm. In the implementation, the FD-PUL parameters were handled using the proposed algorithm in Chapter 6 to be modeled by lumped PUL networks, so that the generalized TEs in (7.8) could be formulated. For a comparison purpose, the transmission lines were also modeled using the conventional methodology, i.e., series lumped models followed by PRIMA. Further, the constructed models based on the proposed algorithm were tested for transient analysis.

\subsubsection{Example 1}

The TL in this example has three conductors with length $2 \mathrm{~cm}$. The PUL resistance and inductance matrices are frequency-dependent and shown in Table 7.1. The PUL conductance 
Table 7.1: Frequency-dependent resistance and inductance matrices. The unit for the PUL parameters are $k \Omega / m$ and $\mu H / m$, respectively. $\left(R_{11}=R_{22}, L_{11}=L_{22}, R_{12}=R_{21}\right.$, $\left.L_{12}=L_{21}\right)$.

\begin{tabular}{|c|c|c|c|c|}
\hline Freq.(GHz) & $R_{11}$ & $R_{12}$ & $L_{11}$ & $L_{12}$ \\
\hline \hline $1.0000 \mathrm{e}-9$ & $2.3490 \mathrm{e}-3$ & $4.6980 \mathrm{e}-4$ & 0.4700 & 0.0940 \\
\hline $1.0000 \mathrm{e}-5$ & $2.3518 \mathrm{e}-3$ & $4.7035 \mathrm{e}-4$ & 0.4695 & 0.0939 \\
\hline $5.1795 \mathrm{e}-4$ & $2.6005 \mathrm{e}-3$ & $5.2010 \mathrm{e}-4$ & 0.4063 & 0.0813 \\
\hline $1.5999 \mathrm{e}-3$ & $2.7213 \mathrm{e}-3$ & $5.4425 \mathrm{e}-4$ & 0.3884 & 0.0777 \\
\hline $1.1514 \mathrm{e}-2$ & $3.2846 \mathrm{e}-3$ & $6.5691 \mathrm{e}-4$ & 0.3699 & 0.0740 \\
\hline $1.4564 \mathrm{e}-1$ & $8.2621 \mathrm{e}-3$ & $1.6524 \mathrm{e}-3$ & 0.3606 & 0.0721 \\
\hline $5.9636 \mathrm{e}-1$ & $1.6604 \mathrm{e}-2$ & $3.3209 \mathrm{e}-3$ & 0.3575 & 0.0715 \\
\hline $1.3895 \mathrm{e}+0$ & $2.5348 \mathrm{e}-2$ & $5.0695 \mathrm{e}-3$ & 0.3560 & 0.0712 \\
\hline $1.8421 \mathrm{e}+0$ & $2.9172 \mathrm{e}-2$ & $5.8344 \mathrm{e}-3$ & 0.3557 & 0.0711 \\
\hline $2.4421 \mathrm{e}+0$ & $3.3585 \mathrm{e}-2$ & $6.7170 \mathrm{e}-3$ & 0.3553 & 0.0711 \\
\hline $3.2375 \mathrm{e}+0$ & $3.8674 \mathrm{e}-2$ & $7.7348 \mathrm{e}-3$ & 0.3550 & 0.0710 \\
\hline $4.2919 \mathrm{e}+0$ & $4.4540 \mathrm{e}-2$ & $8.9080 \mathrm{e}-3$ & 0.3548 & 0.0710 \\
\hline $5.6899 \mathrm{e}+0$ & $5.1296 \mathrm{e}-2$ & $1.0259 \mathrm{e}-2$ & 0.3545 & 0.0709 \\
\hline $1.0000 \mathrm{e}+1$ & $6.8024 \mathrm{e}-2$ & $1.3605 \mathrm{e}-2$ & 0.3541 & 0.0708 \\
\hline
\end{tabular}

and capacitance matrices are given by

$$
\boldsymbol{C}=\left[\begin{array}{cc}
140 & -28 \\
-28 & 140
\end{array}\right] p f / m, \quad \boldsymbol{G}=\left[\begin{array}{ll}
0 & 0 \\
0 & 0
\end{array}\right]
$$

To produce a reduced-order model, the proposed algorithm was run by first fitting the variation in the $\boldsymbol{R}(s)$ and $\boldsymbol{L}(s)$ parameters using a $3 / 2$ order rational function, which was then synthesized into an equivalent lumped network. Figure 7.2 depicts a sample of the variation in those parameters versus frequency with the behavior of the equivalent network. The driving impedance for the above network was written in the form of (7.3) with a dimension $n_{Z}=25$. Similarly, the PUL admittance was written in the form of (7.5) with a dimension $n_{Y}=4$. The procedure presented in Section 7.2.2 was then used to develop the reducedorder model. With the configuration of $H=8, s_{o}=6.28 \times 10^{9}$ and $\epsilon_{\mathcal{S}}=\left\{\left.0.5 \%\right|_{s=6.5 G H z}\right\}$, a reduced system with order 34 was obtained. Figure 7.3 depicts a comparison for $\left|Y_{11}\right|$ 
parameter of the network computed from the reduced-order system and the exact value obtained through the exponential stamp. Figure 7.3 shows that the reduced system is accurate up to $10 G H z$.

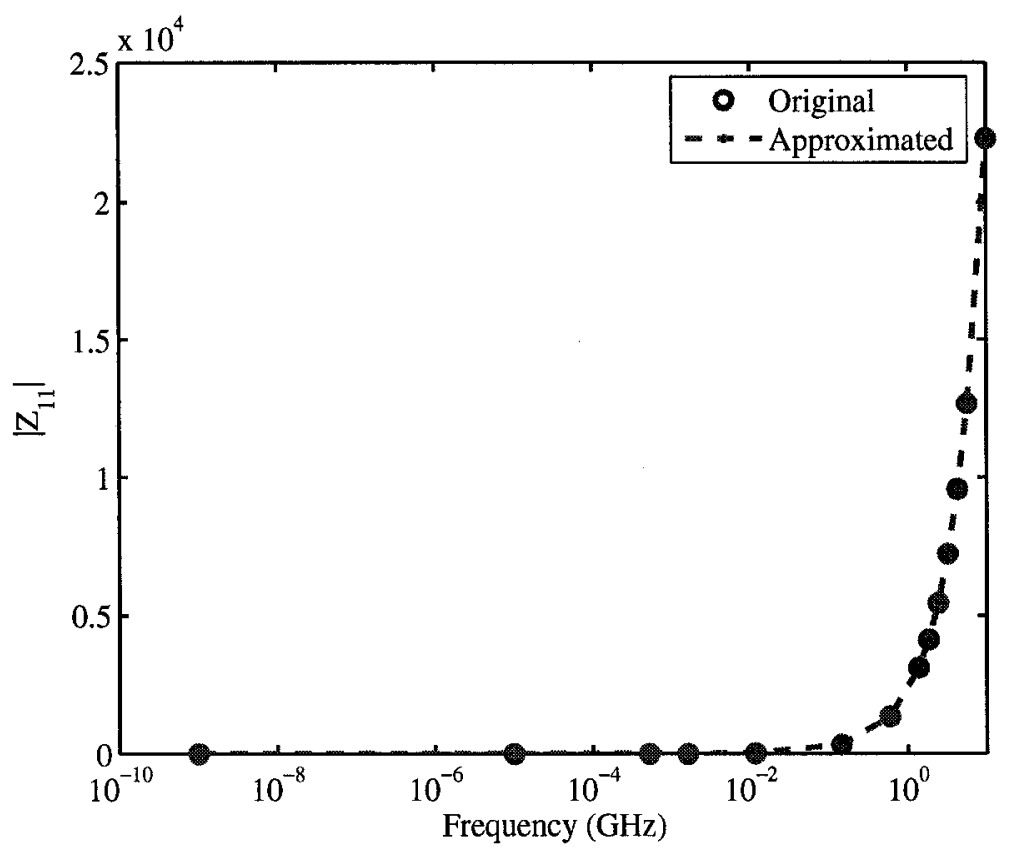

Figure 7.2: A sample of the PUL impedance behavior along with the behavior of the equivalent network.

The above TL was reconsidered using the PRIMA algorithm for the purpose of providing a meaningful comparison. First, the TL interconnect was approximated with 100 sections of RLCM components resulting in a system with order 2506 . This is compared to 264 , the size of the matrix $\left[\boldsymbol{A}_{D}+\frac{2}{d}\left(\boldsymbol{I}_{H+1} \otimes \tilde{\boldsymbol{T}}\right)\right]$ used in the proposed algorithm. A reduced-order system of size 34 was then obtained for the lumped network via the PRIMA algorithm under the same configuration as specified above. Figure 7.4 shows the relative errors incurred by the ICT-based and PRIMA-based reduced models in approximating the interconnect $\left|Y_{11}\right|$ parameter. It is clear from Figure 7.4 that for the size of the reduced system, the model based on ICT shows better approximation especially at lower frequency. The relatively "large" error for the PRIMA-based reduced-order model comes from the 


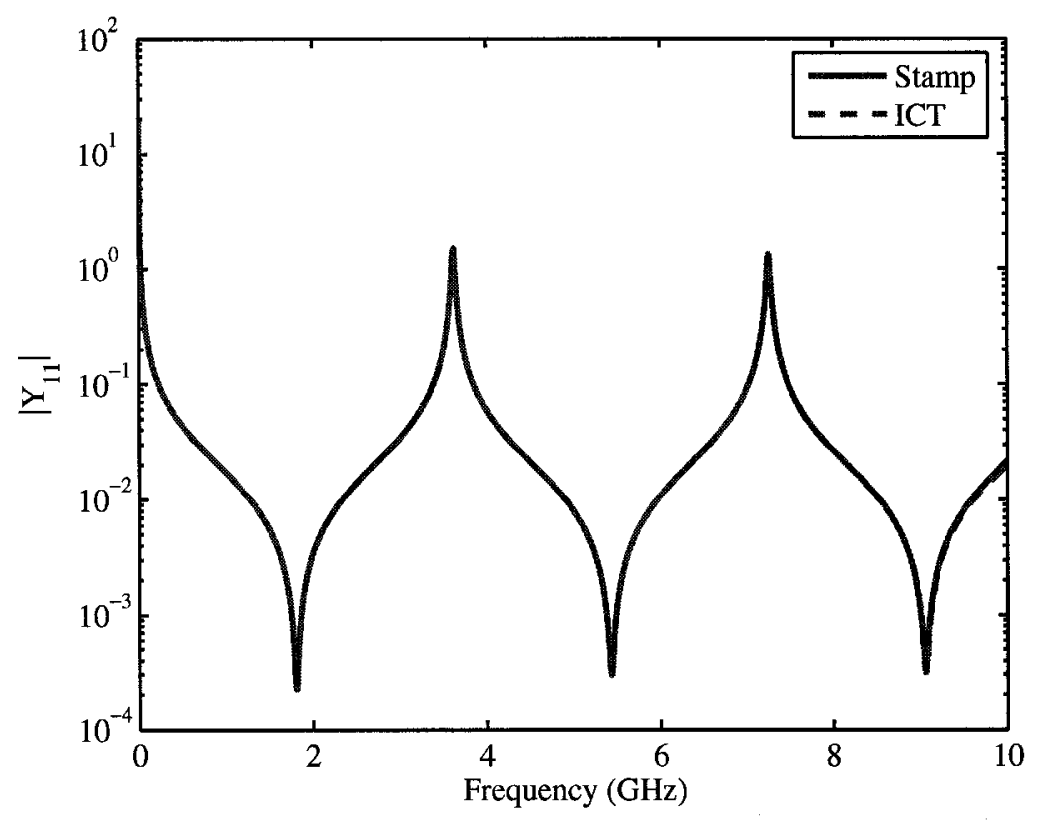

Figure 7.3: Comparison of the ICT model and the TL stamp (Example 1).

lump segmentation for the TL. This error cannot be improved by increasing the order of the reduced system for a specific lumped segmentation.

\subsubsection{Example 2}

The above TL in Example 1 was embedded in two different circuit topologies as shown in Figure 7.5 and Figure 7.6, respectively. The reduced-order model obtained from Section 7.4.1 based on the proposed approach was used to perform the time-domain transient analysis. The circuit in Figure 7.5 was excited by a unit step input voltage source with a rise time of $0.2 \mathrm{~ns}$. Figure 7.7 illustrates the time-domain response of the circuit based on the constructed ICT model. The response was compared to that based on IFFT. It is found that both responses are in good agreement. The second circuit shown in Figure 7.6 was excited by a unit input current pulse of rise/fall time of $0.2 \mathrm{~ns}$ and a pulse-width of 1ns. Figures 7.8(a) and 7.8(b) demonstrate the comparison for the time-domain responses at the far end of active and victim lines between the proposed ICT algorithm and IFFT. Two 


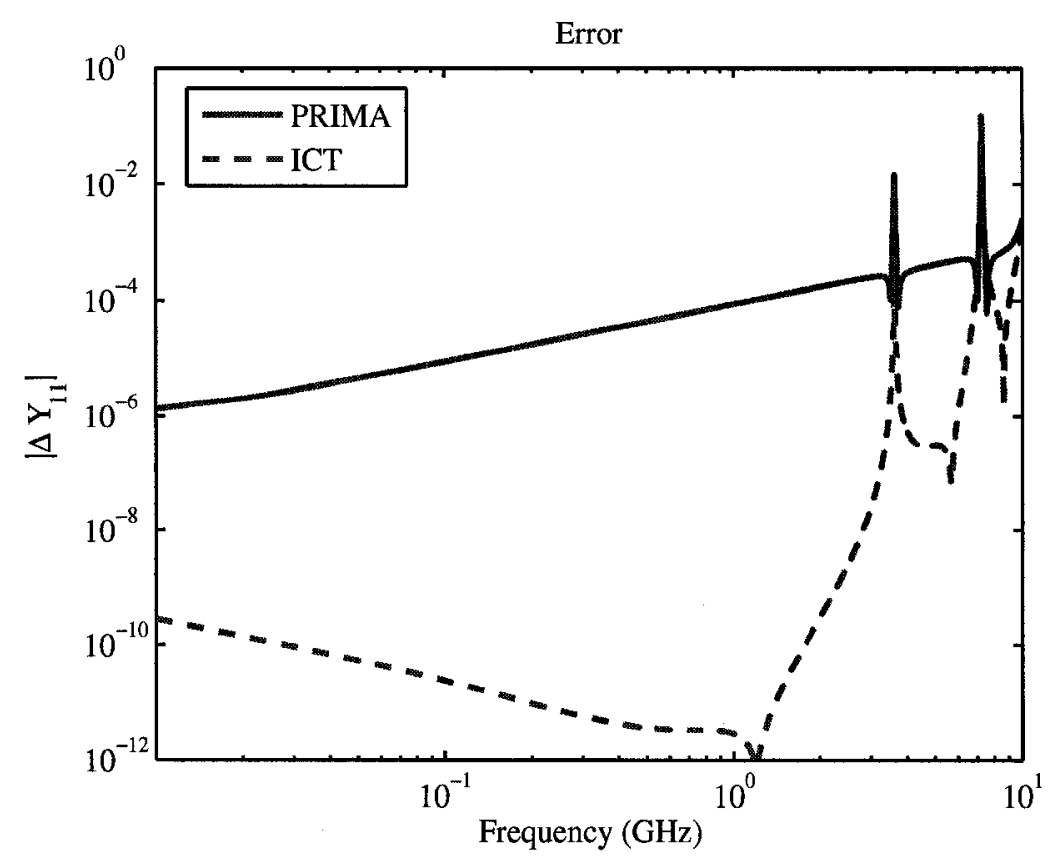

Figure 7.4: Error comparison between the PRIMA and ICT models (Example 1).

curves match well as displayed in Figure 7.8.

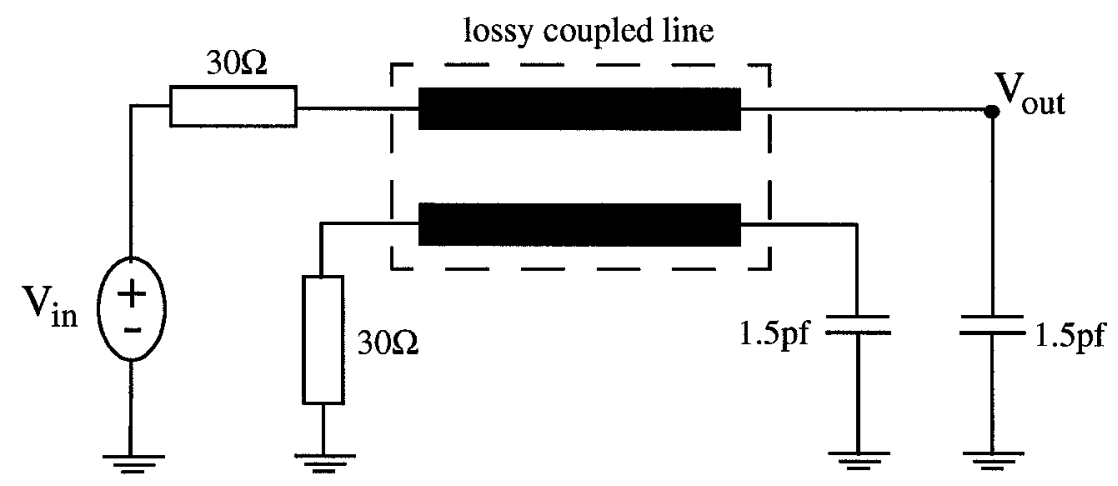

Figure 7.5: Circuit topology-1 for Example 2.

\subsubsection{Example 3}

This example deals with a PCB interconnect network. The frequency-dependent PUL parameters of the TL can be found in [85] referred to as Line 6. The frequency-dependent 


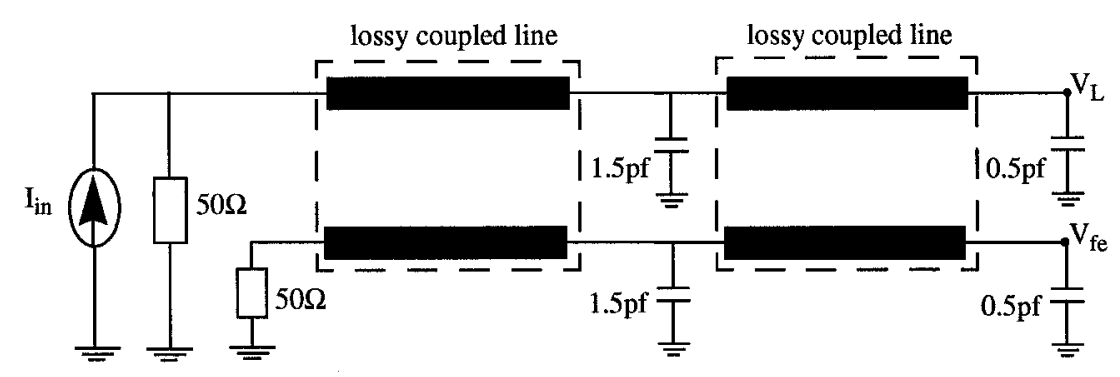

Figure 7.6: Circuit topology-2 for Example 2.

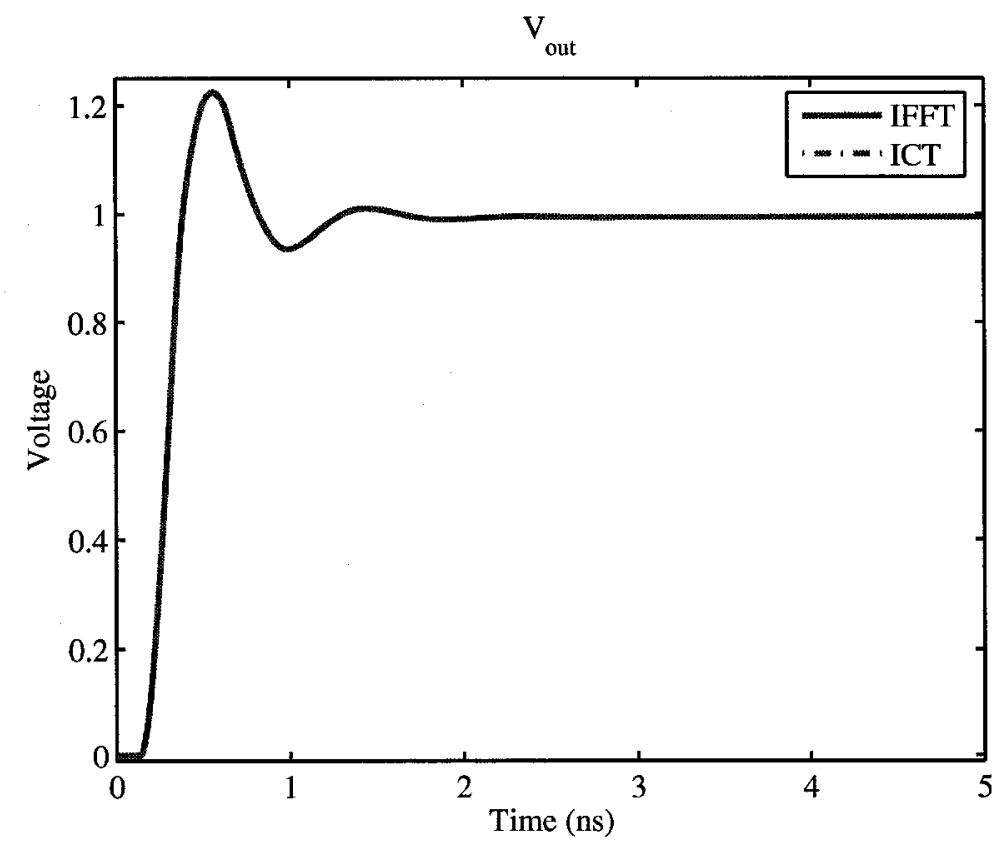

Figure 7.7: Time domain responses for the circuit network in Figure 7.5. 


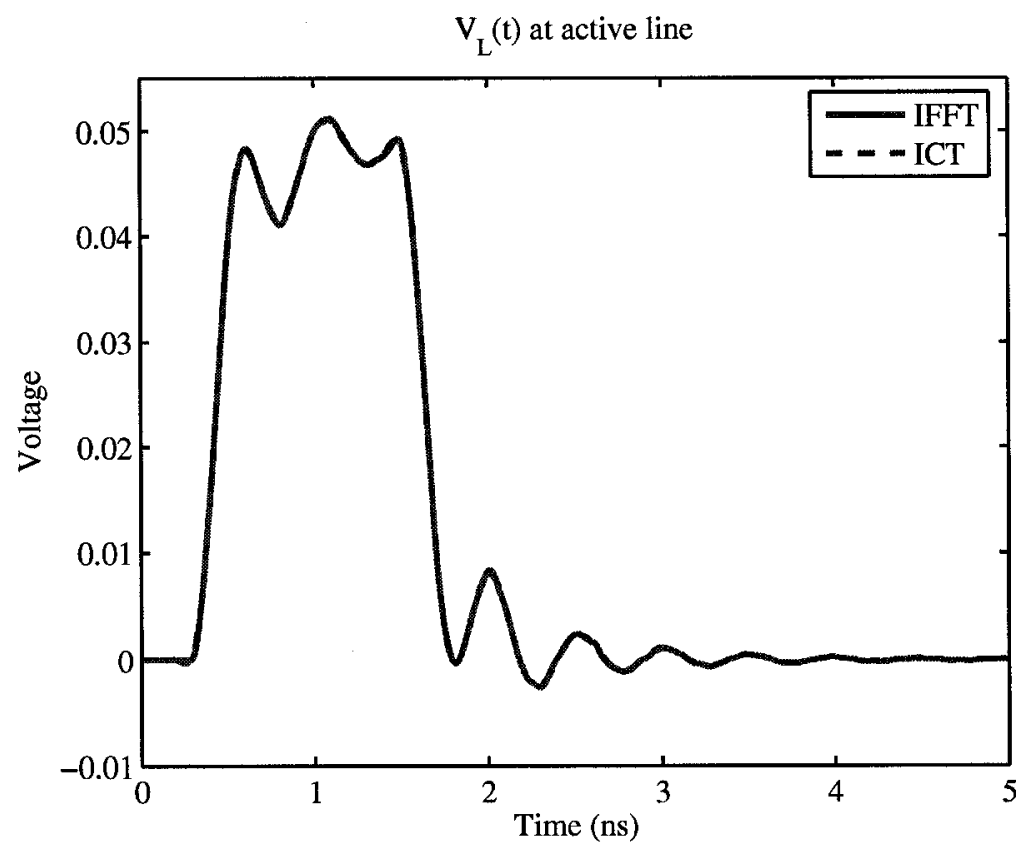

(a) Time domain responses at the active line.

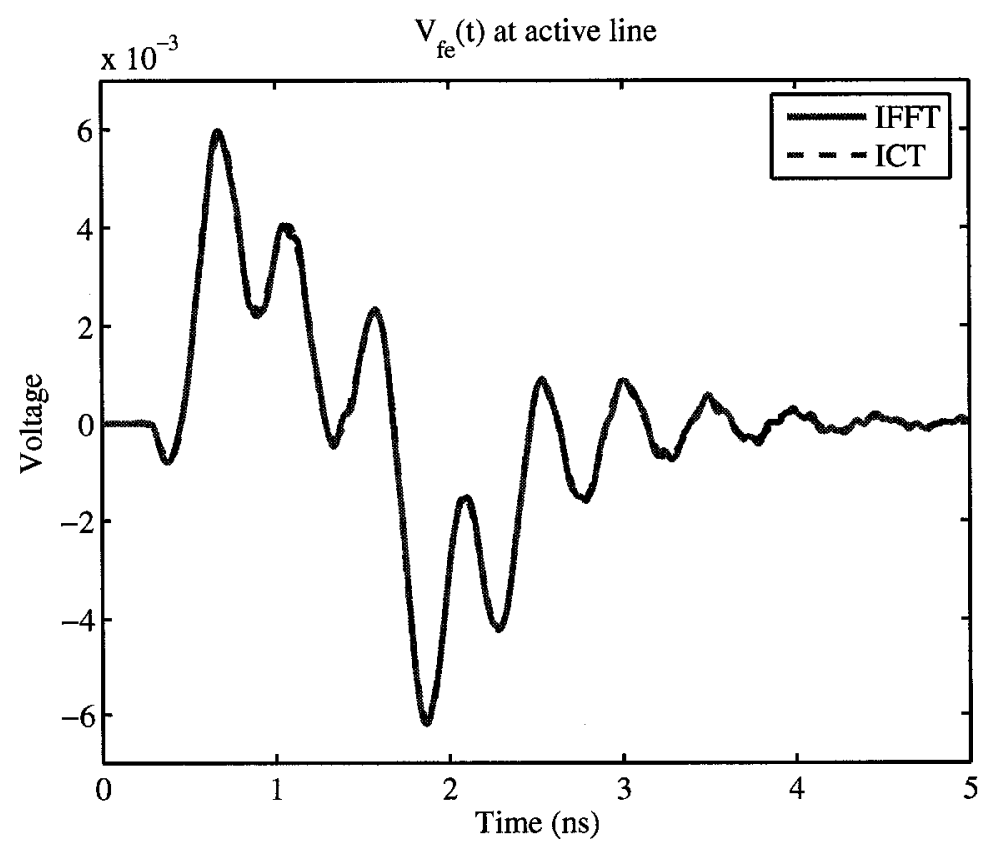

(b) Time domain responses at the victim line.

Figure 7.8: Time domain responses for the circuit network in Figure 7.6. 
PUL impedance and admittance of the TL were approximated with $4 / 3$ and $4 / 3$ order rational functions, respectively, which were synthesized into two equivalent networks. The proposed algorithm was then used to develop a reduced-order model of size 121 via using $20^{\text {th }}$ order Chebyshev polynomials in constructing the $x$-dependent orthonormal basis $\mathcal{Q}(x)$. The expansion point was given by $s_{o}=25 \times 10^{9}$, and the program terminated when the relative error at $16.5 \mathrm{GHz}$ became less than $1 \%$. The $\left|Y_{11}\right|$ parameter of the resulting model is compared to the exact value in Figure 7.9. The two curves in the figure show good agreement.

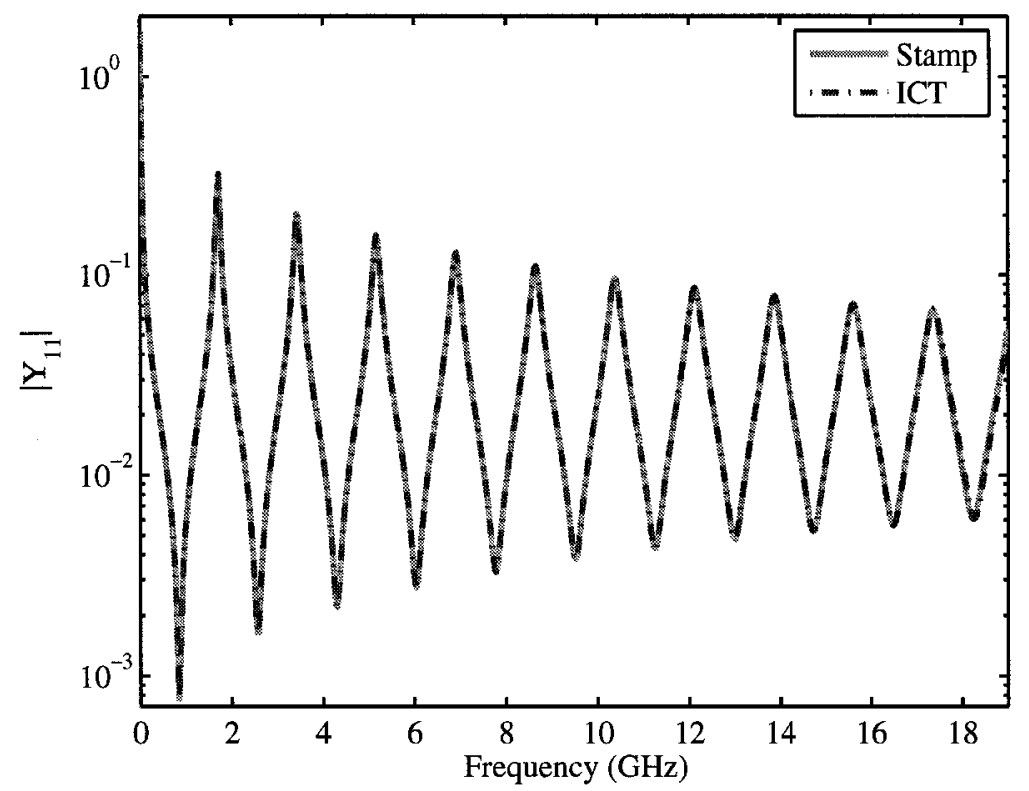

Figure 7.9: Comparison of the ICT model and the TL stamp (Example 3).

The PRIMA algorithm was again used to construct a reduced-order model for the above PCB line. By using 100 lumped sections to approximate the TL as the intermediate step and taking the same expansion point and error criterion as the proposed technique used, a reduced-order model of size 123 was resulted. Figure 7.10 shows the error comparison between the above two obtained reduced models relative to the TL stamp. The comparison 
is consistent with the one in Figure 7.4. It is noted here that the dimension of the system matrices used to construct the orthonormal basis are 4006 and 960 , in respective order, for using the PRIMA and the proposed techniques.

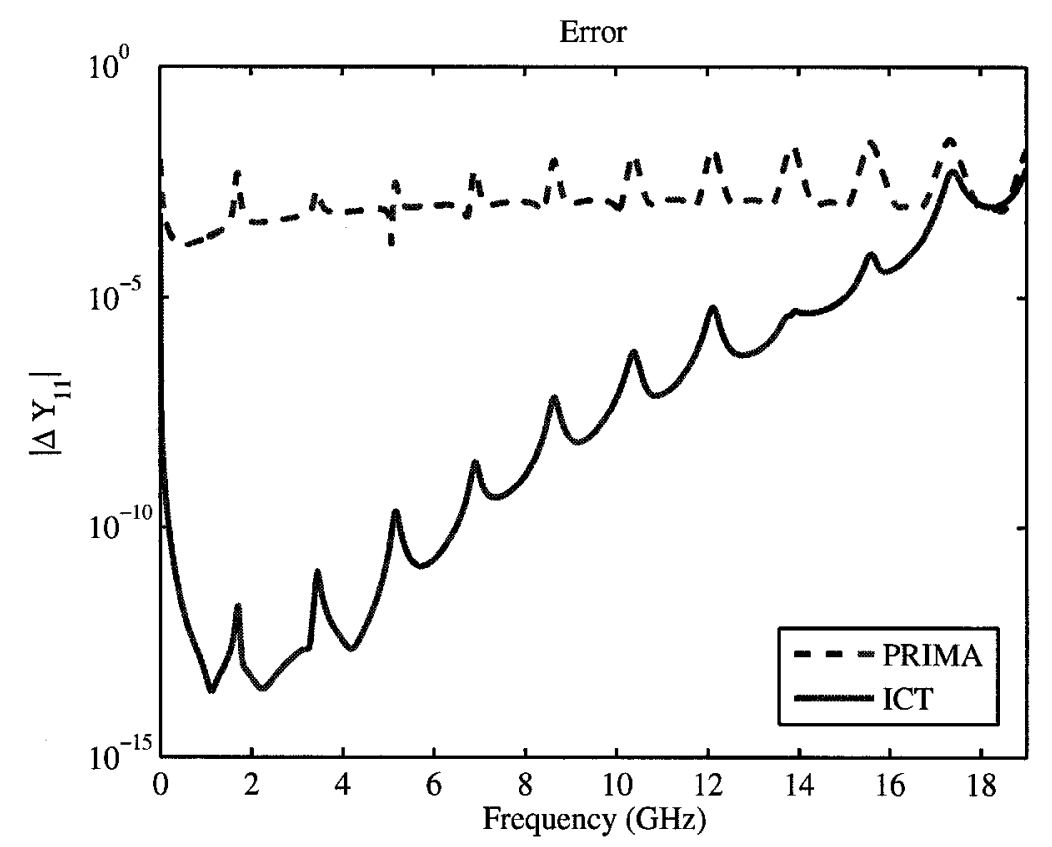

Figure 7.10: Error comparison between the PRIMA and ICT models (Example 3).

The resulting reduced-order model based on ICT was then used to perform the transient analysis for the circuit in Figure 7.11. The input voltage of the circuit network is a unit step response with a rise time of 0.035 ns. Figure 7.12(a) and Figure 7.12(b) compare the transient responses from using IFFT and the ICT model for the active and victim lines, respectively. 


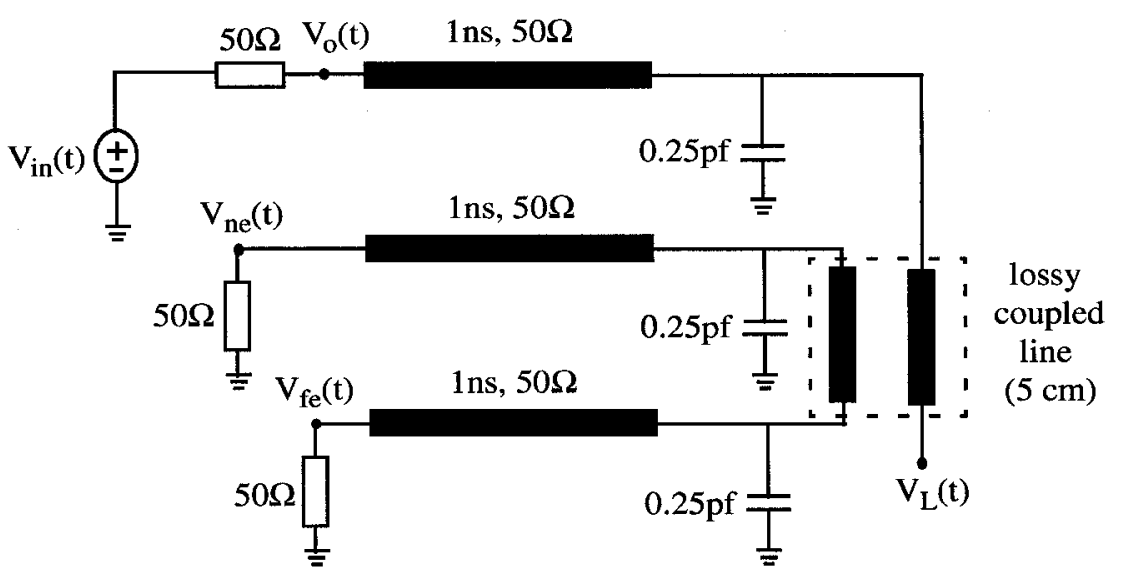

Figure 7.11: Circuit topology for Example 3. 


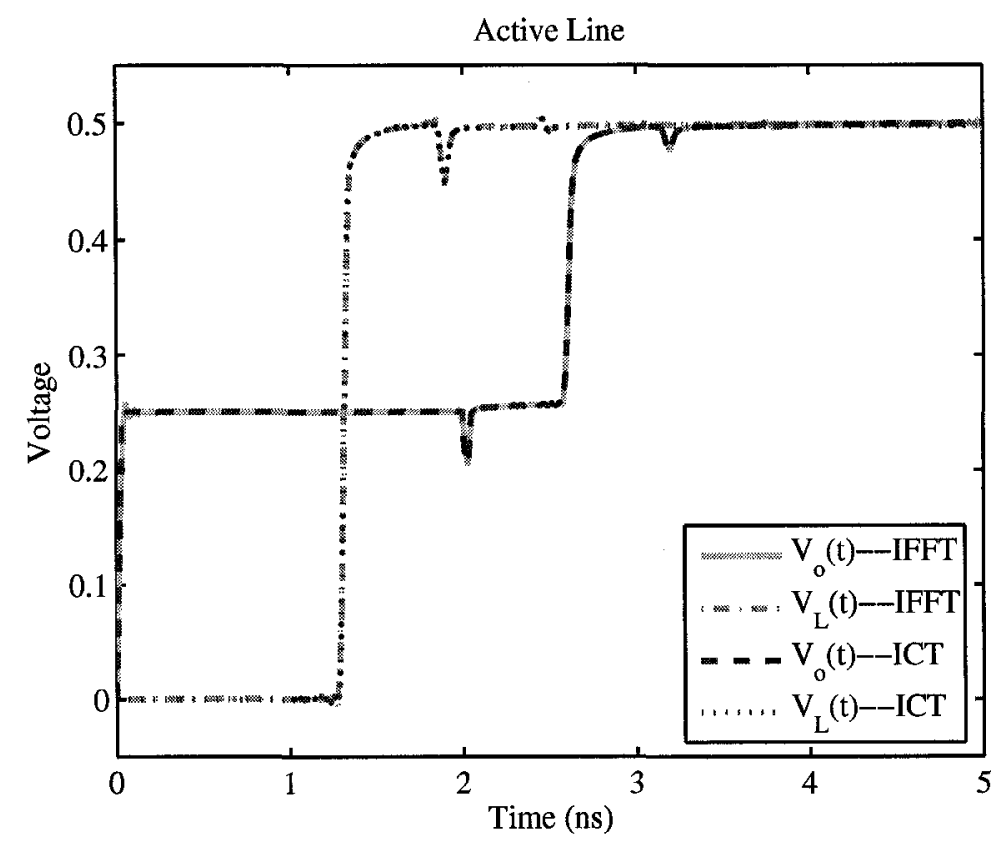

(a) Time domain responses at the active line.

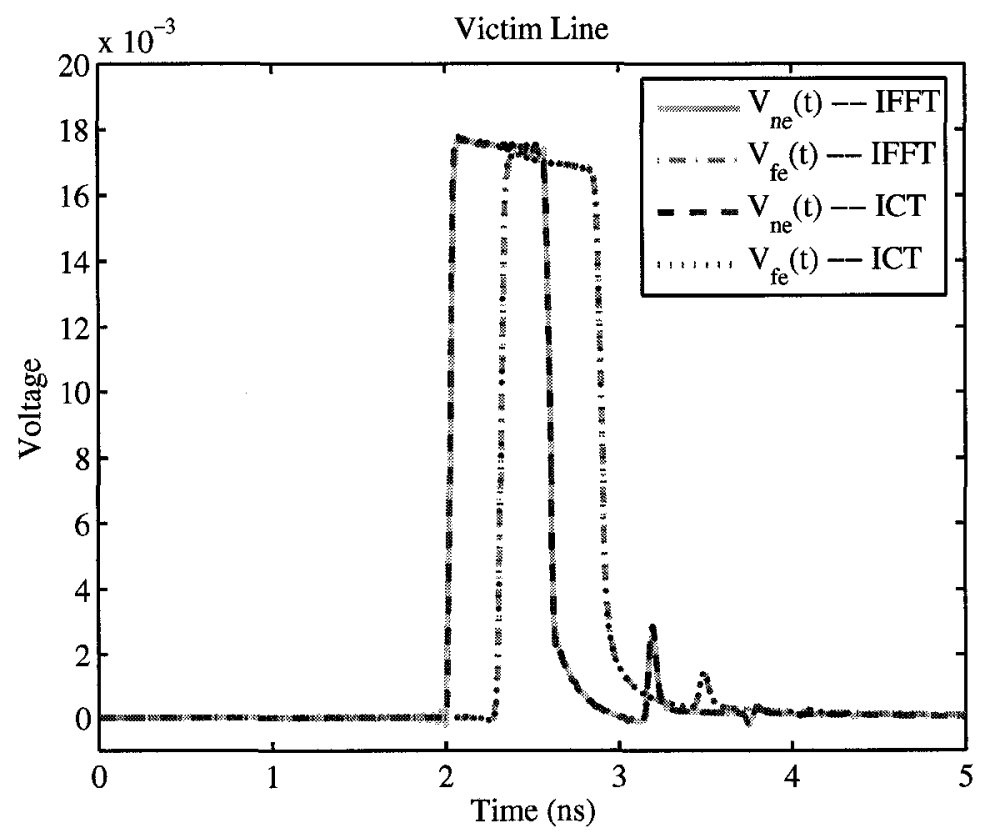

(b) Time domain responses at the victim line.

Figure 7.12: Time domain responses for the PCB interconnect network. 


\section{Chapter 8}

\section{Passive Model-Order Reduction for RLC Circuits with Delay Elements}

\subsection{Introduction}

Chapter 2 describes the Arnoldi-based MOR techniques. It is clear from the presentation that Arnoldi-based MOR in its current form cannot handle situations where the large network includes delay elements. This is evident by the fact that a lumped network is represented by a mixed set of differential-algebraic equations, whereas having delay elements embedded within the network leads to a more general formulation represented by algebraicdelay-differential equations. This situation happens when delay-extraction-based modeling approach, such as DEPACT, is adopted to model the interconnects. Two immediate problems incur when applying Arnoldi-based techniques to an ADDEs system. The foremost challenging issue is the problem of passivity preservation. MOR algorithms in their current form have been derived to tackle the problem of reducing large linear circuits, but with no delay elements. It is therefore not obvious whether the notion of MOR is feasible for networks containing delay elements or, in case it is feasible, how it can be implemented in a way that guarantees the passivity in the reduced-order system. Another important issue, 
that needs to be addressed in the context of MOR, is the computation of the orthonormal basis to be used in the reduction process. The Arnoldi algorithm has been widely used to construct such an orthonormal basis due to its robust numerical performance. The Arnoldi algorithm is essentially an adaptation of the well-known modified Gram-Schmidt process for networks having only lumped components with no delay elements. The presence of delay elements, however, does not permit a straightforward adaptation of MGS to an Arnoldi-like algorithm with same desirable numerical properties.

This chapter presents a new algorithm for MOR of networks with delay elements which addresses the above issues [40]. It is worth mentioning that the idea of conducting MOR on delay systems has been addressed in the control area. More specifically, $H_{\infty}, \mathrm{t}_{2}$ and $\mathrm{Ł}_{\infty}$ controller-based order reduction techniques were proposed recently in $[115,116]$. These techniques, however, depend on solving linear matrix inequalities, which is time consuming for large systems, in addition to the fact that a solution for LMIs can never be guaranteed. The difficulties in these techniques usually stem from defining the reduction error criterion over the entire frequency range, while in interconnects, one is typically interested in having the reduced-order model match the original system over a limited frequency band of interest. Another major disadvantage in these techniques is that they do not address the issue of passivity.

The rest of this chapter is organized as follows. Section 8.2 discusses the context in which networks with delay elements are encountered. Section 8.3 presents the outline in developing the proposed algorithm. Section 8.4 describes the proposed Arnoldi-based construction of the orthonormal basis. Section 8.5 presents some numerical examples to validate the performance of the proposed algorithm. 


\subsection{Networks with delay elements}

The situation, where delay dependent sources are part of the original network, occurs if a delay extraction-based macromodeling approach [10] has been adopted to model certain portions of the interconnects which are described as uniform transmission lines and characterized by low Ohmic losses. The delay sources arising from this macromodeling approach are typically described in the form of lossless coupled MTLs. Figure 8.1 depicts this situation schematically, where the lossless TLs embedded within the original network are typically represented with the delay sources.

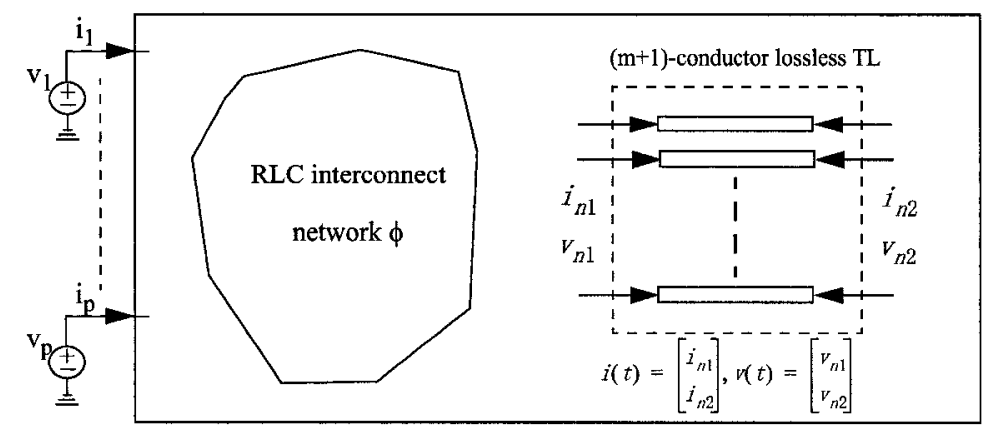

Figure 8.1: A p-port RLC network with a lossless TL.

Without loss of generality, we assume that the original network has a single set of lossless TL with $m+1$ conductors. Thus the system in Figure 8.1 can be described as follows,

$$
\begin{aligned}
\boldsymbol{C}_{\phi} \dot{\boldsymbol{x}}_{\phi}(t)+\boldsymbol{G}_{\phi} \boldsymbol{x}_{\phi}(t)+\mathfrak{S} \boldsymbol{i}_{t l}(t) & =\boldsymbol{b}_{\phi} \boldsymbol{v}_{p}(t) \\
\boldsymbol{i}_{p}(t) & =\boldsymbol{b}_{\phi}^{T} \boldsymbol{x}_{\phi}(t)
\end{aligned}
$$

where $\boldsymbol{C}_{\phi}, \boldsymbol{G}_{\phi}, \boldsymbol{b}_{\phi}, \boldsymbol{x}_{\phi}(t), \boldsymbol{v}_{p}(t)$ and $\boldsymbol{i}_{p}(t)$ have the same definitions similar to (2.23). $\mathfrak{S}$ is a selector matrix which maps port currents of the lossless MTL, $i_{t l}(t)$, into the node space of $\boldsymbol{x}_{\phi}(t)$ [1]. The fundamental formalism given in [1,9] can be used here to describe the 
relation between the voltages and currents at the ports of the lossless TL in the Laplacedomain. This gives us

$$
\left\{\begin{array}{l}
\boldsymbol{V}_{n 1}(s)=\mathcal{Z}_{o} \boldsymbol{I}_{n 1}(s)+e^{-s \sqrt{\boldsymbol{L C}} d}\left(\mathcal{Z}_{o} \boldsymbol{I}_{n 2}(s)+\boldsymbol{V}_{n 2}(s)\right) \\
\boldsymbol{V}_{n 2}(s)=\mathcal{Z}_{o} \boldsymbol{I}_{n 2}(s)+e^{-s \sqrt{L \boldsymbol{C}} d}\left(\mathcal{Z}_{o} \boldsymbol{I}_{n 1}(s)+\boldsymbol{V}_{n 1}(s)\right)
\end{array}\right.
$$

where $L$ and $C$ represent the PUL inductance and capacitance of the lossless TL with $d$ being the length of the TL. $\mathcal{Z}_{o}$ is the characteristic admittance of the TL defined by $\mathcal{Z}_{o}=$ $\boldsymbol{L} \sqrt{\boldsymbol{L} \boldsymbol{C}}^{-1} . \boldsymbol{I}_{n 1}(s)\left(\boldsymbol{I}_{n 2}(s)\right)$ and $\boldsymbol{V}_{n 1}(s)\left(\boldsymbol{V}_{n 2}(s)\right)$ represent the near_ (far_) end Laplacedomain currents and voltages, respectively, whereas their time-domain counterparts, i.e., $\boldsymbol{i}_{n 1}(t)\left(\boldsymbol{i}_{n 2}(t)\right)$ and $\boldsymbol{v}_{n 1}(t)\left(\boldsymbol{v}_{n 2}(t)\right)$, constitute the vectors $\boldsymbol{i}_{t l}(t)$ and $\boldsymbol{v}_{t l}(t)$, i.e.,

$$
\boldsymbol{i}_{t l}(t)=\left[\begin{array}{c}
\boldsymbol{i}_{n 1}(t) \\
\boldsymbol{i}_{n 2}(t)
\end{array}\right], \boldsymbol{v}_{t l}(t)=\left[\begin{array}{l}
\boldsymbol{v}_{n 1}(t) \\
\boldsymbol{v}_{n 2}(t)
\end{array}\right]
$$

Thus, $\boldsymbol{v}_{t l}(t)$ is a subset of the state variables in $\boldsymbol{x}_{\phi}(t)$ and it satisfies $\boldsymbol{v}_{t l}(t)=\mathfrak{S}^{T} \boldsymbol{x}_{\phi}(t)$.

\subsection{Development of the proposed algorithm}

The proposed algorithm can be described as a two-stage process. The first stage, which is presented in Section 8.3.1, formulates the network with delay elements as a set of ADDEs. The developed formulation represents the key idea in guaranteeing the passivity of the reduced-order system. The second stage builds on this formulation to construct the reduced-order system, which possesses the same delay based structure of the original one in the form of ADDEs. This part is given in Section 8.3.2. Section 8.3.3 presents the proof of passivity of the reduced-order model. 


\subsubsection{Formulation of ADDEs}

The following proposition describes an important step needed in deriving the desired ADDEs for the considered network.

Proposition 8.3.4 For a $(m+1)$-conductor lossless $T L$, the relation between the port currents and voltages can be described in the time-domain using the following system of difference equations,

$$
\begin{aligned}
\mathbf{0} & =\boldsymbol{A} \varrho(t)+\sum_{k=1}^{m} \boldsymbol{A}_{k} \varrho\left(t-\tau_{k}\right)+\boldsymbol{B} \boldsymbol{i}_{t l}(t) \\
\boldsymbol{v}_{t l}(t) & =\boldsymbol{B}^{T} \varrho(t)
\end{aligned}
$$

Furthermore, if we let $\hat{\Pi}(s)$ denote the transfer function of the above system, then $\hat{\Pi}(s)$ represents the impedance matrix of the MoC model for the lossless MTL and is defined by

$$
\hat{\boldsymbol{\Pi}}(s)=\boldsymbol{B}^{T}\left(-\boldsymbol{A}-\sum_{k=1}^{m} \boldsymbol{A}_{k} e^{-s \tau_{k}}\right)^{-1} \boldsymbol{B}
$$

where,

$$
\begin{aligned}
& \boldsymbol{A}_{k}=\frac{\mathfrak{M}}{2}\left[\begin{array}{cccc}
0 & -\boldsymbol{e}_{k} & \mathbf{0} & -\boldsymbol{e}_{k} \\
-\boldsymbol{e}_{k} & \mathbf{0} & -\boldsymbol{e}_{k} & \mathbf{0} \\
\mathbf{0} & \boldsymbol{e}_{k} & \mathbf{0} & \boldsymbol{e}_{k} \\
\boldsymbol{e}_{k} & \mathbf{0} & \boldsymbol{e}_{k} & \mathbf{0}
\end{array}\right] \mathfrak{M}^{-1} \mathcal{Z}_{o} \\
& \boldsymbol{A}=\left[\begin{array}{cccc}
-\frac{\mathcal{Z}_{o}}{2} & 0 & \frac{\mathcal{Z}_{o}}{2} & \mathbf{0} \\
\mathbf{0} & -\frac{\boldsymbol{Z}_{o}}{2} & \mathbf{0} & \frac{\boldsymbol{Z}_{o}}{2} \\
-\frac{\mathcal{Z}_{o}}{2} & 0 & -\frac{\mathcal{Z}_{o}}{2} & 0 \\
0 & -\frac{\mathcal{Z}_{o}}{2} & \mathbf{0} & -\frac{\mathcal{Z}_{o}}{2}
\end{array}\right], \quad \boldsymbol{B}=\left[\begin{array}{cc}
0 & 0 \\
0 & 0 \\
\mathcal{Z}_{o} & 0 \\
\mathbf{0} & \mathcal{Z}_{o}
\end{array}\right]
\end{aligned}
$$

$\boldsymbol{e}_{k}$ is an $m \times m$ zero matrix, except that the $(k, k)^{\text {th }}$ element is " 1 "; $\mathcal{Z}_{o}$ is the characteristic impedance of the $T L ; \mathfrak{M}$ is the matrix of eigenvectors of the matrix $\sqrt{L C} d$ with $\tau_{k}, k=$ $1, \ldots, m$ being the corresponding eigenvalues.

The proof of the above proposition is given in Appendix F. Proposition 8.3.4 represents the enabling idea that allows formulating the entire system (including the delay elements) 
in a single system of ADDEs. This system takes the following form,

$$
\begin{aligned}
\tilde{\mathcal{K}} \dot{\tilde{\varrho}}(t) & =\tilde{\mathcal{A}} \tilde{\varrho}(t)+\sum_{k=1}^{m} \tilde{\mathcal{A}}_{k} \tilde{\boldsymbol{\varrho}}\left(t-\tau_{k}\right)+\tilde{\mathcal{B}} v_{p}(t) \\
\boldsymbol{i}_{p}(t) & =\tilde{\mathcal{B}}^{T} \tilde{\varrho}(t)
\end{aligned}
$$

where

$$
\begin{gathered}
\tilde{\mathcal{K}}=\left[\begin{array}{ccc}
\boldsymbol{C}_{\phi} & \mathbf{0} & 0 \\
\mathbf{0} & \mathbf{0} & 0 \\
\mathbf{0} & \mathbf{0} & 0
\end{array}\right], \tilde{\mathcal{A}}=-\left[\begin{array}{ccc}
\boldsymbol{G}_{\phi} & \mathbf{0} & \mathfrak{S} \\
\mathbf{0} & -\boldsymbol{A} & -\boldsymbol{B} \\
-\mathfrak{S}^{T} & \boldsymbol{B}^{T} & \mathbf{0}
\end{array}\right], \\
\tilde{\mathcal{A}}_{k}=-\left[\begin{array}{ccc}
0 & 0 & 0 \\
0 & -\boldsymbol{A}_{k} & \mathbf{0} \\
\mathbf{0} & \mathbf{0} & \mathbf{0}
\end{array}\right], \tilde{\mathcal{B}}=\left[\begin{array}{c}
\boldsymbol{b}_{\phi} \\
\mathbf{0} \\
\mathbf{0}
\end{array}\right], \tilde{\boldsymbol{\varrho}}(t)=\left[\begin{array}{c}
\boldsymbol{x}_{\phi}(t) \\
\tilde{\boldsymbol{\varrho}}(t) \\
\boldsymbol{i}_{t l}(t)
\end{array}\right] \in \mathbb{R}^{N_{d}}
\end{gathered}
$$

with $N_{d}=N_{\phi}+6 m$. The next subsection demonstrates how to construct the reduced-order system from the ADDEs shown in (8.5).

\subsubsection{Construction of the reduced-order systems}

Rewriting the ADDEs (8.5) in the Laplace-domain results in

$$
\begin{aligned}
s \tilde{\mathcal{K}}_{\boldsymbol{}}(s) & =\tilde{\mathcal{A}} \boldsymbol{\varsigma}(s)+\sum_{k=1}^{m} \tilde{\mathcal{A}}_{k} \boldsymbol{\varsigma}(s) e^{-s \tau_{k}}+\tilde{\mathcal{B}} \boldsymbol{V}_{p}(s) \\
\boldsymbol{I}_{p}(s) & =\tilde{\mathcal{B}}^{T} \boldsymbol{\varsigma}(s)
\end{aligned}
$$

where, $\boldsymbol{\varsigma}(s), \boldsymbol{V}_{p}(s)$ and $\boldsymbol{I}_{p}(s)$ are the Laplace-domain representations for $\tilde{\varrho}(t), \boldsymbol{v}_{p}(t)$ and $i_{p}(t)$, respectively. Performing the MOR on the above system requires computing an orthonormal basis for the first $\left\lfloor\frac{n}{p}\right\rfloor$ block moments of $\varsigma(s)$, i.e., its first $\left\lfloor\frac{n}{p}\right\rfloor$ scaled derivatives w.r.t. s. If such an orthonormal basis is denoted by $\mathcal{Q} \in \mathbb{R}^{N_{d} \times q}$, then the reduced-order system would then be given by

$$
\begin{aligned}
\hat{\mathcal{K}} \dot{\hat{\varrho}}(t) & =\hat{\mathcal{A}} \hat{\varrho}(t)+\sum_{k=1}^{m} \hat{\mathcal{A}}_{k} \hat{\varrho}\left(t-\tau_{k}\right)+\hat{\mathcal{B}} \boldsymbol{v}_{p}(t) \\
i_{p}(t) & =\hat{\mathcal{B}}^{T} \hat{\varrho}(t)
\end{aligned}
$$

where,

$$
\hat{\mathcal{K}}=\mathcal{Q}^{T} \tilde{\mathcal{K}} \mathcal{Q}, \hat{\mathcal{A}}=\mathcal{Q}^{T} \tilde{\mathcal{A}} \mathcal{Q}, \hat{\mathcal{A}}_{k}=\mathcal{Q}^{T} \tilde{\mathcal{A}}_{k} \mathcal{Q}, \hat{\mathcal{B}}=\mathcal{Q}^{T} \tilde{\mathcal{B}}, \text { and } \hat{\varrho}(t) \text { is a vector }
$$


containing the state variables in the reduced domain.

With a smaller order compared to (8.5), the reduced-order system (8.7) can be solved efficiently using numerical integration techniques for the transient analysis. Construction of the orthonormal basis, $\mathcal{Q}$, represents a key issue in obtaining a compact reduced-order model. This issue will be addressed in Section 8.4.

\subsubsection{Passivity of the reduced-order system}

It is well-known that a provably passive macromodel represents the ultimate guarantee of the stability of transient time-domain simulation under arbitrary loading conditions. A system is passive if and only if its admittance/impedance (or immittance) matrix satisfies those three conditions of PR-ness listed in Section 2.2.

As discussed previously, the three conditions can be simplified to only two conditions (the second and third conditions) for systems with no delay elements. The reason behind this simplification is that in delay-free systems the immittance matrix can always be put in a rational matrix form [45]. In this particular case, having the third condition satisfied automatically implies that the first condition, i.e., the analyticity in the right-half plane, is also satisfied [14]. However, the type of system considered here in (8.5) is a delay-based system. This fact makes its characteristic polynomial of the transcendental type [93], which does not permit a rational function representation using a finite number of poles. In fact, the concept of poles can not be defined in this context. Therefore, a proof of passivity in this situation will have to proceed by considering the original three conditions without the initial simplification used in classical projection-based MOR of lumped systems. The above observation is incorporated in the following theorem and the proof of passivity provided afterwards. 
Theorem 8.3.7 The admittance matrix of the reduced-order system given by

$$
\tilde{\boldsymbol{Y}}(s)=\hat{\mathcal{B}}^{T}\left(s \hat{\mathcal{K}}-\hat{\mathcal{A}}-\sum_{k=1}^{m} \hat{\mathcal{A}}_{k} e^{-s \tau_{k}}\right)^{-1} \hat{\mathcal{B}}
$$

satisfies the three conditions of PR-ness and therefore the reduced-order system is passive.

It is easy to see that $\tilde{\boldsymbol{Y}}(s)$ satisfies the second condition by noting that matrices $\hat{\mathcal{B}}, \hat{\mathcal{K}}$, $\hat{\mathcal{A}}$ and $\hat{\mathcal{A}}_{k}$ are all real matrices. The proof that $\tilde{\boldsymbol{Y}}(s)$ satisfies the other two conditions will be given through the following theorems and their proofs.

Theorem 8.3.8 $\left[\tilde{\boldsymbol{Y}}(s)+\tilde{\boldsymbol{Y}}^{T}\left(s^{\star}\right)\right]$ is non-negative definite for all $s$ in the right half plane of s-domain and therefore satisfies the third condition of passivity.

The following lemma is needed in the proof of Theorem 8.3.8.

Lemma 8.3.9 The matrix $\left(-\boldsymbol{A}-\sum_{k=1}^{m} \boldsymbol{A}_{k} e^{-s \tau_{k}}-\boldsymbol{A}^{T}-\sum_{k=1}^{m} \boldsymbol{A}_{k}^{T} e^{-s^{\star} \tau_{k}}\right)$ is non-negative definite for all sin the right half plane of s-domain.

The proof of Lemma 8.3.9 is given in Appendix G.

Proof (Proof of Theorem 8.3.8) In order to prove that $\left[\tilde{\boldsymbol{Y}}(s)+\tilde{\boldsymbol{Y}}^{T}\left(s^{\star}\right)\right]$ is non-negative definite, we need to show that (8.9) is true for all $z \in \mathbb{R}^{n}$ and for all $s$ with $\Re\{s\}>0$.

$$
\boldsymbol{z}^{* T}\left[\tilde{\boldsymbol{Y}}(s)+\tilde{\boldsymbol{Y}}^{T}\left(s^{\star}\right)\right] \boldsymbol{z} \geq 0
$$

Substituting (8.8) into (8.9) and using some mathematical manipulations, we obtain

$$
\begin{aligned}
\boldsymbol{z}^{\star T} & {\left[\tilde{\boldsymbol{Y}}(s)+\tilde{\boldsymbol{Y}}^{T}\left(s^{\star}\right)\right] \boldsymbol{z} } \\
& =\hat{\boldsymbol{z}}^{\star T}\left[s \tilde{\mathcal{K}}-\tilde{\mathcal{A}}-\sum_{k=1}^{m} \tilde{\mathcal{A}}_{k} e^{-s \tau_{k}}+s^{\star} \tilde{\mathcal{K}}^{T}-\tilde{\mathcal{A}}^{T}-\sum_{k=1}^{m} \tilde{\mathcal{A}}_{k}^{T} e^{-s^{\star} \tau_{k}}\right] \hat{\boldsymbol{z}}
\end{aligned}
$$

where $\hat{\boldsymbol{z}}=\mathcal{Q}\left[s^{\star} \hat{\mathcal{K}}^{T}-\hat{\mathcal{A}}^{T}-\sum_{k=1}^{m} \hat{\mathcal{A}}_{k}^{T} e^{-s^{\star} \tau_{k}}\right]^{-1} \hat{\mathcal{B}} \boldsymbol{z}$. 
Let $s=\sigma+\jmath \omega, \sigma>0$, then

$$
s \tilde{\mathcal{K}}+s^{\star} \tilde{\mathcal{K}}^{T}=2 \sigma\left[\begin{array}{ccc}
C_{\phi} & 0 & 0 \\
\mathbf{0} & \mathbf{0} & \mathbf{0} \\
\mathbf{0} & \mathbf{0} & \mathbf{0}
\end{array}\right] \geq 0
$$

The above inequality means that $s \tilde{\mathcal{K}}+s^{\star} \tilde{\mathcal{K}}^{T}$ is non-negative definite matrix. This result follows as a consequence of having $C_{\phi}$ a non-negative definite matrix. Next consider the matrix, $-\tilde{\mathcal{A}}-\sum_{k=1}^{m} \tilde{\mathcal{A}}_{k} e^{-s \tau_{k}}-\tilde{\mathcal{A}}^{T}-\sum_{k=1}^{m} \tilde{\mathcal{A}}_{k}^{T} e^{-s^{\star} \tau_{k}}$, which can be put in the following block diagonal form,

$$
\begin{aligned}
-\tilde{\mathcal{A}}-\sum_{k=1}^{m} \tilde{\mathcal{A}}_{k} e^{-s \tau_{k}}-\tilde{\mathcal{A}}^{T}-\sum_{k=1}^{m} \tilde{\mathcal{A}}_{k}^{T} e^{-s^{\star} \tau_{k}} & \\
& =\left[\begin{array}{ccc}
\boldsymbol{G}_{\phi}+\boldsymbol{G}_{\phi}^{T} & \mathbf{0} & \mathbf{0} \\
\mathbf{0} & -\boldsymbol{A}-\sum_{k=1}^{m} \boldsymbol{A}_{k} e^{-s \tau_{k}}-\boldsymbol{A}^{T}-\sum_{k=1}^{m} \boldsymbol{A}_{k}^{T} e^{-s^{\star} \tau_{k}} & \mathbf{0} \\
\mathbf{0} & \mathbf{0} & \mathbf{0}
\end{array}\right]
\end{aligned}
$$

It can be easily shown that $\boldsymbol{G}_{\phi}+\boldsymbol{G}_{\phi}^{T}$ is non-negative definite. It follows from Lemma 8.3.9 that the left-side matrix in (8.12) is non-negative definite. Consequently, this proves the inequality in (8.9) which proves that $\tilde{\boldsymbol{Y}}(s)$ is PR and satisfies the third condition of passivity.

Theorems 8.3.9 and 8.3.10 presented below demonstrate that $\tilde{\boldsymbol{Y}}(s)$ is analytic in the right-half plane, and therefore satisfies the first condition of passivity.

Theorem 8.3.9 Assume that $\tilde{\boldsymbol{Y}}(s)$ is non-singular within a certain region of the s-domain, then it is also analytic in the same region.

Proof It is noted that $\tilde{\boldsymbol{Y}}(s)$ can be decomposed into a product of three matrices. Two of them are constant. The other one is the inverse of another matrix, which is a summation of analytical matrices. Hence, the proof of the theorem follows directly based on Lemmas 2.2.1-2.2.4 stated in Section 2.2. 
Theorem 8.3.10 If $\left[\tilde{\boldsymbol{Y}}(s)+\tilde{\boldsymbol{Y}}^{T}\left(s^{\star}\right)\right]$ is non-negative definite for all $s$ in the right half plane of s-domain, then it must be non-singular in that plane.

Proof This theorem is proved by contradiction, where we first assume that $\tilde{\boldsymbol{Y}}(\mathrm{s})$ is singular, and then proceed to show that this singularity contradicts the assumption that $[\tilde{\boldsymbol{Y}}(s)+$ $\left.\tilde{\boldsymbol{Y}}^{T}\left(s^{\star}\right)\right]$ is non-negative definite. The singularity assumption implies that $\tilde{\boldsymbol{Y}}(s)$ can be represented by the Laurent series expansion in a small region around the singular point, $p_{o},[49]$,

$$
\tilde{\boldsymbol{Y}}(s)=\sum_{i=0}^{\infty} \boldsymbol{a}_{i}\left(s-p_{o}\right)^{i}+\sum_{i=1}^{\infty} \frac{\boldsymbol{b}_{i}}{\left(s-p_{o}\right)^{i}}
$$

where $\boldsymbol{a}_{i}$ and $\boldsymbol{b}_{i}$ are matrices whose elements contain the expansion coefficients of each element in $\tilde{\boldsymbol{Y}}(s)$. Using a polar form representation, we may write

$$
\begin{aligned}
\left(s-p_{o}\right) & =k_{1} e^{\jmath \theta_{1}} \\
\boldsymbol{b}_{i} & =\boldsymbol{k}_{2} \cdot e^{\jmath \theta_{2}}
\end{aligned}
$$

Here, $\boldsymbol{k}_{2}$ and $\boldsymbol{\theta}_{2}$ are matrices whose elements are the magnitudes and phases, respectively, of each element in $b_{i}$ when written in polar form. Thus, the real part of the dominant term in the expansion (8.13) near the singular point $p_{o}$ will be given by [117],

$$
\Re\left\{\frac{\boldsymbol{b}_{i}}{\left(s-p_{o}\right)^{i}}\right\}=\frac{\boldsymbol{k}_{2} \cdot \cos \left(\boldsymbol{\theta}_{2}-i \theta_{1}\right)}{k_{1}^{i}}
$$

Since $\boldsymbol{k}_{2}$ and $\boldsymbol{\theta}_{2}$ are fixed for a given transfer function, and $\theta_{1}$ varies from 0 to $2 \pi$ as $s$ assumes values in a circular disc around $p_{o}$, each term in (8.16) changes signs $2 i$ times. As a result, in the small neighborhoods around $p_{o}$ in the right-half plane, the real part of $\tilde{Y}(s)$ cannot be considered neither positive nor negative definite matrix. This conclusion is in contradiction with the initial assumption made in the theorem statement, and therefore 
the theorem is proved.

Proof (Proof of Theorem 8.3.7) This proof follows directly from Theorems 8.3.8, 8.3.9 and 8.3.10, which show that $\tilde{\boldsymbol{Y}}(s)$ satisfies the first and third conditions of passivity.

\subsection{Arnoldi-based construction of the orthonormal basis}

Computation of the orthonormal basis $\mathcal{Q}$ represents a key issue in having an accurate reduced-order system over a wide frequency range. In fact, accurate orthonormal basis impacts the size of the reduced system, making it smaller, which consequently reduces the CPU time spent during the transient time-domain simulations. MGS is a general purpose procedure that can be used to construct an orthonormal basis for the subspace spanned by a set of vectors. To use MGS in constructing $\mathcal{Q}$, one would have first to compute the moments of $\varsigma(s)$. These moments can be obtained by expanding $\varsigma(s)$ in a Taylor series,

$$
\varsigma(s)=\sum_{i=0}^{\infty} \boldsymbol{M}_{i} s^{i}
$$

and then substituting from (8.17) into (8.6), using the Taylor series expansion of the exponential factors, and equating similar powers of $s$ to obtain the following recursive relation for the moments,

$$
\begin{aligned}
\boldsymbol{M}_{0} & =\boldsymbol{E}_{0}^{-1} \tilde{\mathcal{B}} \\
\boldsymbol{M}_{i} & =-\boldsymbol{E}_{0}^{-1} \sum_{j=1}^{i} \boldsymbol{E}_{j} \boldsymbol{M}^{i-j} \quad i \geq 1
\end{aligned}
$$

where $\boldsymbol{E}_{j}$ represents the coefficients of $s^{j}$ in the Taylor series of $\left(s \tilde{\mathcal{K}}-\tilde{\mathcal{A}}-\sum_{k=1}^{m} \tilde{\mathcal{A}}_{k} e^{-s \tau_{k}}\right)$,

$$
\left(s \tilde{\mathcal{K}}-\tilde{\mathcal{A}}-\sum_{k=1}^{m} \tilde{\mathcal{A}}_{k} e^{-s \tau_{k}}\right)=\sum_{j=0}^{\infty} \boldsymbol{E}_{j} s^{j}
$$


Recursive computation of the moments using the above relation incurs unacceptable numerical error, especially in high-order moments. This error typically arises from having the small round-off errors that result from finite precision calculation of the low order moments get amplified in the process of computing the high-order moments. In fact, it can be seen from (8.19) that the amplification factor is proportional in magnitude of the largest eigenvalue of the matrix $\boldsymbol{E}_{0}^{-1}$. Thus high-order moments tend to have a numerical error that is many orders of magnitude higher than the machine round-off error. This makes any effort to include high order moments in computing $\mathcal{Q}$ practically useless as it does not enhance the accuracy of the reduced-order system.

One way to tackle this problem is based on adopting the Arnoldi algorithm instead of the MGS process to carry out the orthonormalization. In fact, the Arnoldi algorithm is nothing more than an MGS process adapted to the special case of having the vectors, for which an orthonormal basis is desired, related by the following simple recursive relation,

$$
\boldsymbol{r}_{i}=\boldsymbol{\mathcal { T }} \boldsymbol{r}_{i-1}
$$

where $\mathcal{T}$ is some matrix operator. The numerical robustness of the Arnoldi algorithm stems from its stability to obtain the orthonormal basis for the moments, but without computing these moments explicitly. This point represents the key idea in eliminating the amplification of the round-off error in high-order moments.

Unfortunately, adapting MGS to an Arnoldi-like algorithm for the delay system is not a straightforward task, since the moments are not related by the simple recursive relation given by (8.21). In fact, such a relation would only exist if the system was delay-free in which case the matrix $\mathcal{T}$ would be given by $\boldsymbol{G}_{\phi}^{-1} \boldsymbol{C}_{\phi}$. This problem is handled here as follows. We first expand the exponential factors $e^{-s \tau_{k}}$ by their Taylor series expansion 
using their moments,

$$
e^{-s \tau_{k}}=\sum_{i=0}^{\infty} \frac{\left(-\tau_{k}\right)^{i}}{i !} s^{k}
$$

It is important to stress the point that the moments of $e^{-s \tau_{k}}$ are available in a closed-form, $\frac{\left(-\tau_{k}\right)^{i}}{i !}$. Therefore, their computation does not introduce errors beyond the small machinerelated finite precision errors. If we truncate the above Taylor series to only $q$ terms so that it is a good approximation for the original exponential function within a given frequency range of interest, and then use the truncated expansion to substitute into (8.6) for $e^{-s \tau_{k}}$, we obtain the following system

$$
\left[\boldsymbol{\Gamma}_{0}+\boldsymbol{\Gamma}_{1} s+\ldots+\boldsymbol{\Gamma}_{q-1} s^{q-1}\right] \tilde{\boldsymbol{\psi}}(s)=\tilde{\mathcal{B}} V_{p}(s)
$$

where,

$$
\Gamma_{i}= \begin{cases}-\tilde{\mathcal{A}}-\sum_{k=1}^{m} \tilde{\mathcal{A}}_{k} \frac{\left(-\tau_{k}\right)^{i}}{i !} & i=0 \\ \tilde{\mathcal{K}}-\sum_{k=1}^{m} \tilde{\mathcal{A}}_{k} \frac{\left(-\tau_{k}\right)^{i}}{i !} & i=1 \\ -\sum_{k=1}^{m} \tilde{\mathcal{A}}_{k} \frac{\left(-\tau_{k}\right)^{i}}{i !} & i \geq 2\end{cases}
$$

It is not difficult to see that $\tilde{\psi}(s)$ is a good approximation for $\varsigma(s)$ within the frequency range of interest. Therefore, an orthonormal basis for the first $\left\lfloor\frac{n}{p}\right\rfloor$ leading block moments of $\tilde{\psi}(s)$ would approximately span the same subspace of the first $\left\lfloor\frac{n}{p}\right\rfloor$ leading block moments of $\varsigma(s)$. Here, $n$ is a positive number so that any higher-order moment does not enhance the approximation of $\tilde{\psi}(s)$ for $\varsigma(s)$. However, the moments of $\tilde{\psi}(s)$ are not related in a way amenable to using the Arnoldi algorithm for computing the orthonormal basis. To overcome this problem, we formulate (8.23) in an augmented form known as the companion form,

$$
s \mathbb{M} \tilde{\boldsymbol{\eta}}(s)+\mathbb{N} \tilde{\boldsymbol{\eta}}(s)=\mathcal{L} V_{p}(s)
$$


where

$$
\begin{gathered}
\mathbb{N}=\left[\begin{array}{llll}
\boldsymbol{\Gamma}_{0} & & & \\
& -\boldsymbol{I}_{N_{d}} & & \\
& & \ddots & \\
& & & -\boldsymbol{I}_{N_{d}}
\end{array}\right], \quad \mathbb{M}=\left[\begin{array}{llll}
\boldsymbol{\Gamma}_{1} & \boldsymbol{\Gamma}_{2} & \ldots & \boldsymbol{\Gamma}_{q} \\
\boldsymbol{I}_{N_{d}} & & & \\
& \ddots & & \\
& & \boldsymbol{I}_{N_{d}} & \mathbf{0}
\end{array}\right], \\
\mathcal{L}=\left[\begin{array}{c}
\tilde{\mathcal{B}} \\
\mathbf{0} \\
\vdots \\
0
\end{array}\right], \quad \tilde{\boldsymbol{\eta}}(s)=\left[\begin{array}{c}
\tilde{\boldsymbol{\psi}}(s) \\
s \tilde{\boldsymbol{\psi}}(s) \\
\vdots \\
s^{q-1} \tilde{\boldsymbol{\psi}}(s)
\end{array}\right]
\end{gathered}
$$

and $\boldsymbol{I}_{N_{d}}$ is a $N_{d} \times N_{d}$ identity matrix.

Two observations can be made at this point. The first observation is that the first block of $\tilde{\boldsymbol{\eta}}(s)$ is identical to $\tilde{\boldsymbol{\psi}}(s)$. The other observation is that moments of $\tilde{\boldsymbol{\eta}}(s)$ are related recursively via the following relation,

$$
\begin{aligned}
& M_{\tilde{\boldsymbol{\eta}}, 0}=-\mathbb{N}^{-1} \mathcal{L} \\
& M_{\tilde{\boldsymbol{\eta}}, i}=\mathbb{N}^{-1} \mathbb{M}^{-1} M_{\tilde{\boldsymbol{\eta}}, i-1}
\end{aligned}
$$

which is similar to (8.21). Thus computing an orthonormal basis for $M_{\tilde{\eta}, 0}, \ldots, M_{\tilde{\eta}, i}$ can be carried out using the Arnoldi algorithm. Let $\Upsilon$ denote the orthonormal basis of the

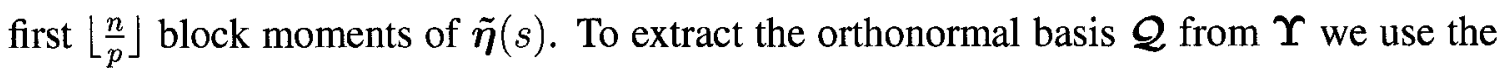
fact that the columns in the first $N_{d} \times n$ block in $\Upsilon$ can be shown to span the subspace of $\varsigma(s)$. A proof of this fact is actually similar to the proof presented in [22]. However, this block is not orthonormal and therefore computing the orthonormal $\mathcal{Q}$ can be carried out by performing another round of orthonormalization. Figure 8.2 presents a detailed pseudocode summarizing the computational steps used to obtain $\mathcal{Q}$.

It should be noted here that although the pseudocode procedure uses the inverse of the augmented matrix in computing $\mathcal{Q}$, in a practical implementation one needs only to 


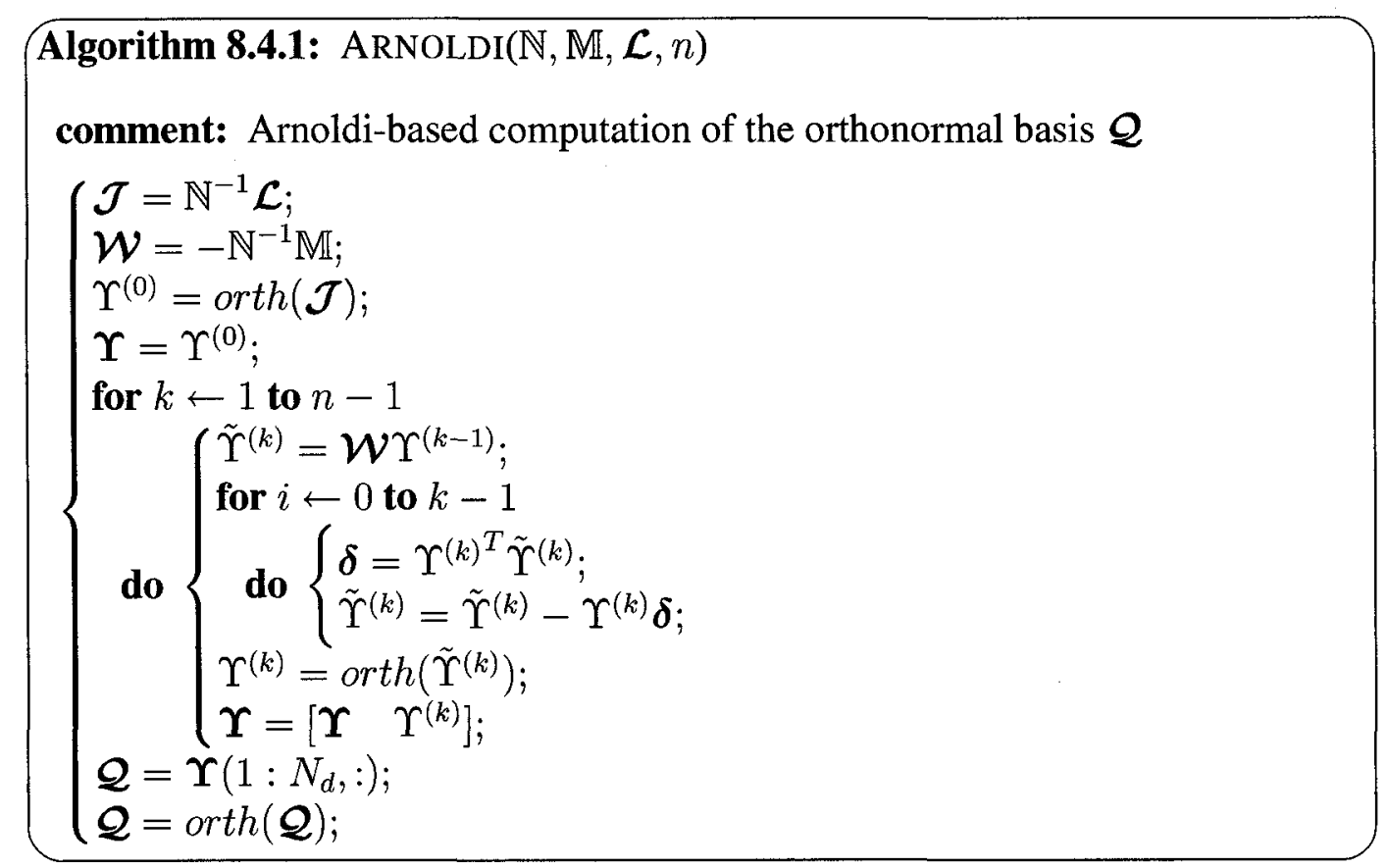

Figure 8.2: Pseudocode for computing the orthonormal basis $\mathcal{Q}$.

perform L/U decomposition on $\Gamma_{0}$, which is much smaller in size, and use F/B substitution with $\tilde{\mathcal{B}}$ (or the first block of $\mathcal{L}$ ) to compute $\mathbb{N}^{-1} \mathcal{L}$ and $\mathbb{N}^{-1} \mathbb{M}$. The $\mathrm{L} / \mathrm{U}$ decomposition will have to be done only once, whereas the F/B substitution needs to be done $q$ times.

\subsection{Numerical examples}

\subsubsection{Validation of the implicit moment technique}

The first example is used to demonstrate the advantage of using the proposed algorithm in constructing the orthonormal basis $\mathcal{Q}$ over using the explicit approach. In this example, a network composed of 20 lossless 3 -conductor TLs, representing the delay elements, and a circuit with lumped RLC components, has been considered for reduction. The size of this network is 2735 . Two approaches were then considered to obtain the reduced-order system. In the first approach, the proposed algorithm outlined in Section 8.4 was adopted to 
construct the orthonormal basis. This approach is labelled "Implicit", and the Y-parameter computed based on the resulting reduced-order system were plotted as the dashed line in Figure 8.3. The figure also shows the accurate Y-parameters computed from the original network in the solid line. The second approach used to generate the reduced-order system was based on using the relations in (8.18)-(8.19) to compute the moments explicitly, where an MGS process was used afterwards to compute the orthonormal basis. This approach was then labelled "Explicit", and the corresponding Y-parameters have been shown in dotted line in Figure 8.3. It is seen from Figure 8.3 that although the basis in both cases were of size 80 , thereby making the size of the reduced-order system 80 as well, the one constructed based on the proposed algorithm matches the original system over a much wider frequency range.

\subsubsection{One-port network}

The second example is a one-port network with lumped RLC components and a lossless 3-conductor TL. An ADDE system as described in Section 8.3.1 was first constructed to describe the entire network with a size of 418 . One reduced-order system of size 20 was obtained with 6 -th order Taylor expansion for the exponential terms. Figure 8.4(a) shows the response obtained from the reduced-order system and compares it with the response of the original system. To validate the performance of the reduced-order system for timedomain transient analysis, the reduced-order model, as well as the original network, were excited with an impulse current source. The rise/fall time, pulse width and magnitude of the source are $0.02 n s, 3 n s$ and $0.02 A$, respectively. A comparison for the voltage responses obtained from the original and reduced systems is given in Figure 8.4(b). 


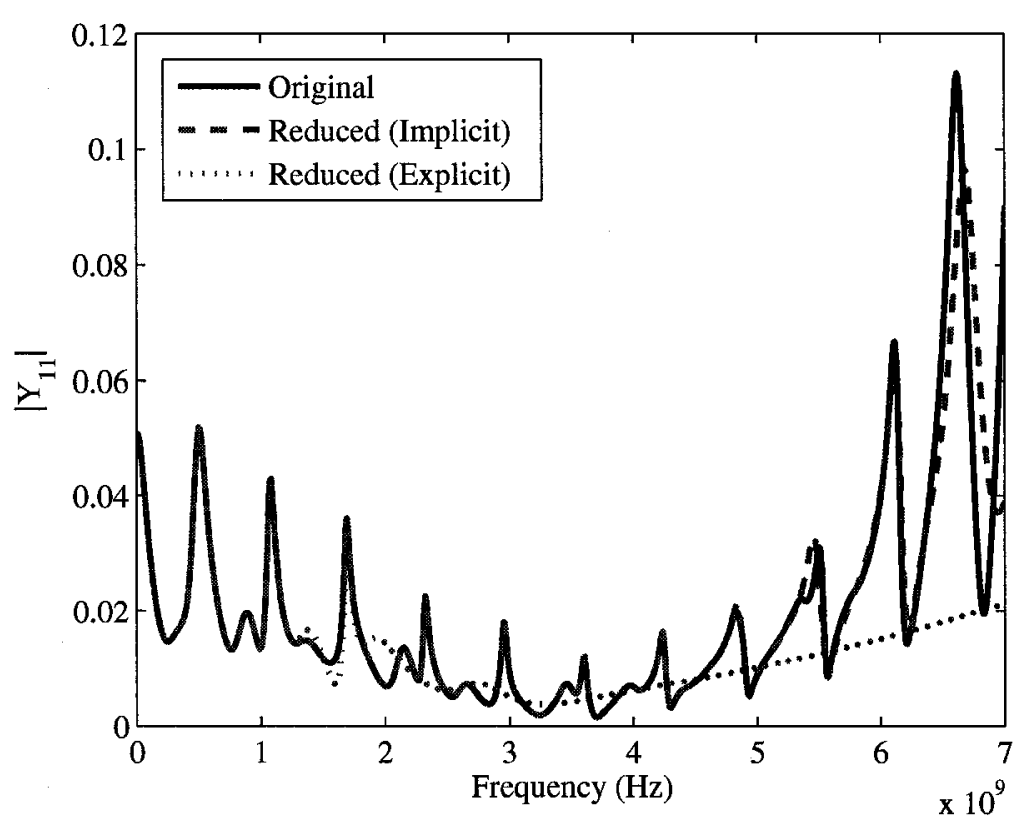

(a) Comparison of the system response $Y_{11}$.

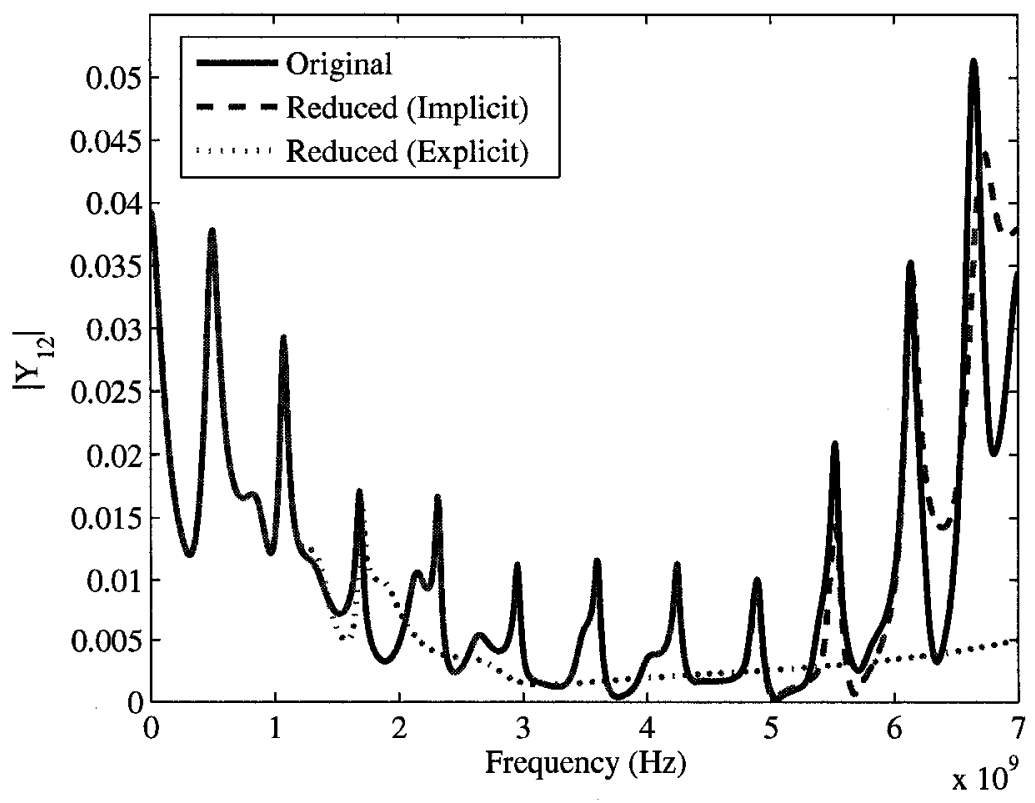

(b) Comparison of the system response $Y_{12}$.

Figure 8.3: Frequency-domain responses (Example 1). 


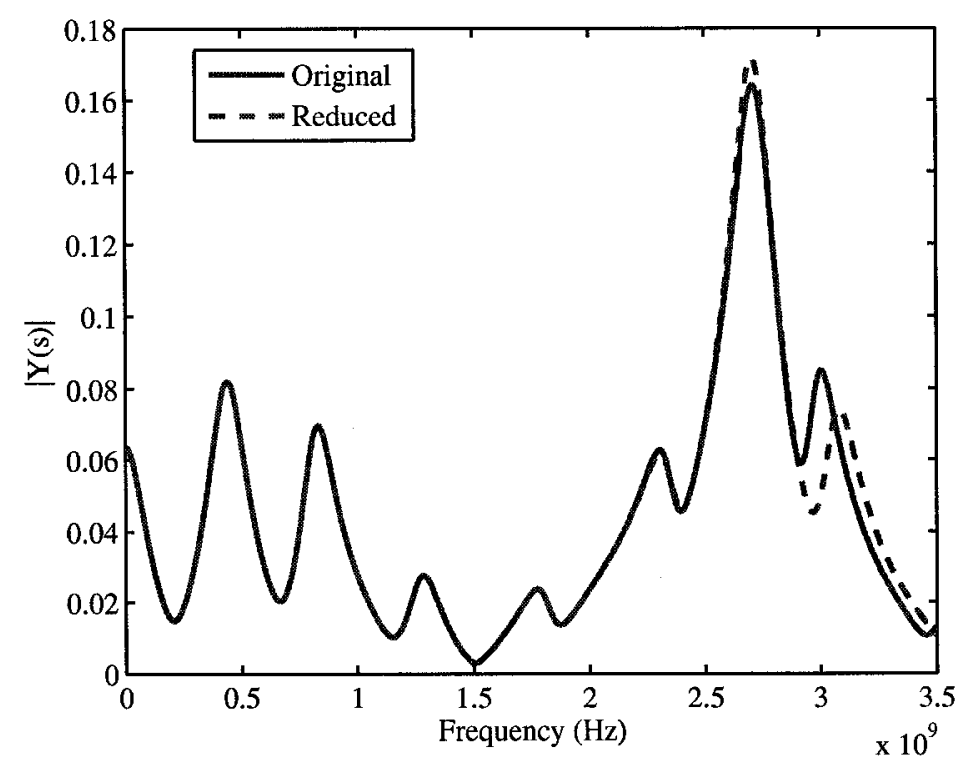

(a) Comparison of the system responses.

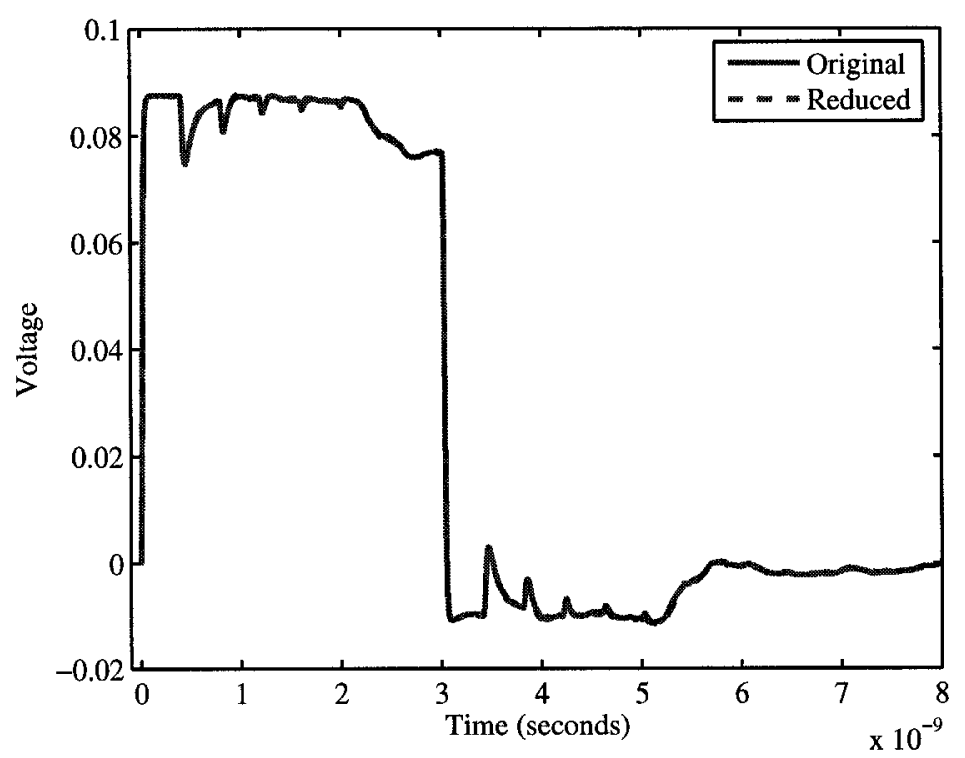

(b) Comparison of transient responses at port voltage.

Figure 8.4: Numerical performance of the reduced-order system (Example 2). 


\subsubsection{Two-port network}

A two-port network consisting of 2097 lumped components and 120 sets of lossless 2conductor TLs, as illustrated in Figure 8.5, is considered in this example. Using the formulation introduced in Section 8.3.1, a system of ADDE of order 2390 was constructed. The proposed algorithm is then used to obtain a reduced-order model for the original network. Figure 8.6 shows the performance of a reduced system of order 60 in the frequency-domain when the exponential terms are approximated by 4-th order Taylor series expansions.

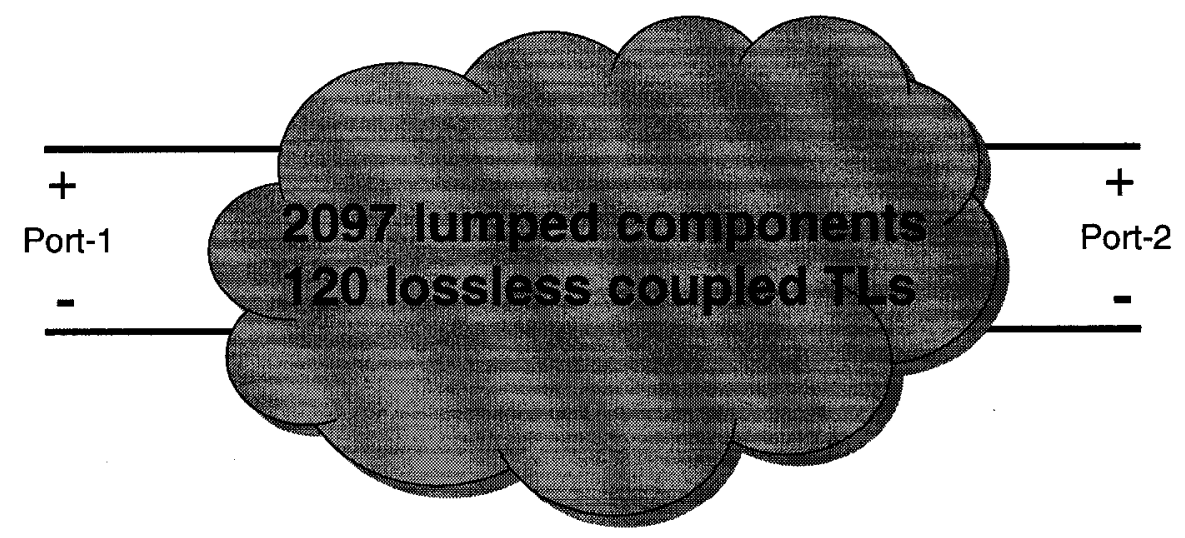

Figure 8.5: A two-port network (Example 3).

The above network was excited by an input pulse of rise/fall time of $0.05 \mathrm{~ns}$ and a pulse width of $3 \mathrm{~ns}$ at one port, while the other port was terminated with a $50 \Omega$ resistor. Figure 8.7 demonstrates the comparison for the time-domain responses obtained using the reduced-order model and the original circuit.

Table 8.1 summarizes the CPU speedup in performing the transient analysis using the proposed algorithm as compared to simulating the original system. The original system was simulated in HSPICE ${ }^{\circledR}$ while the reduced system was simulated by transient solver. Both simulations were run on a Sun Solaris platform with a UltraSPARC-IIIi processor $(1.5 \mathrm{GHz})$. It is worth to pointing out that the numerical integration for the original system 


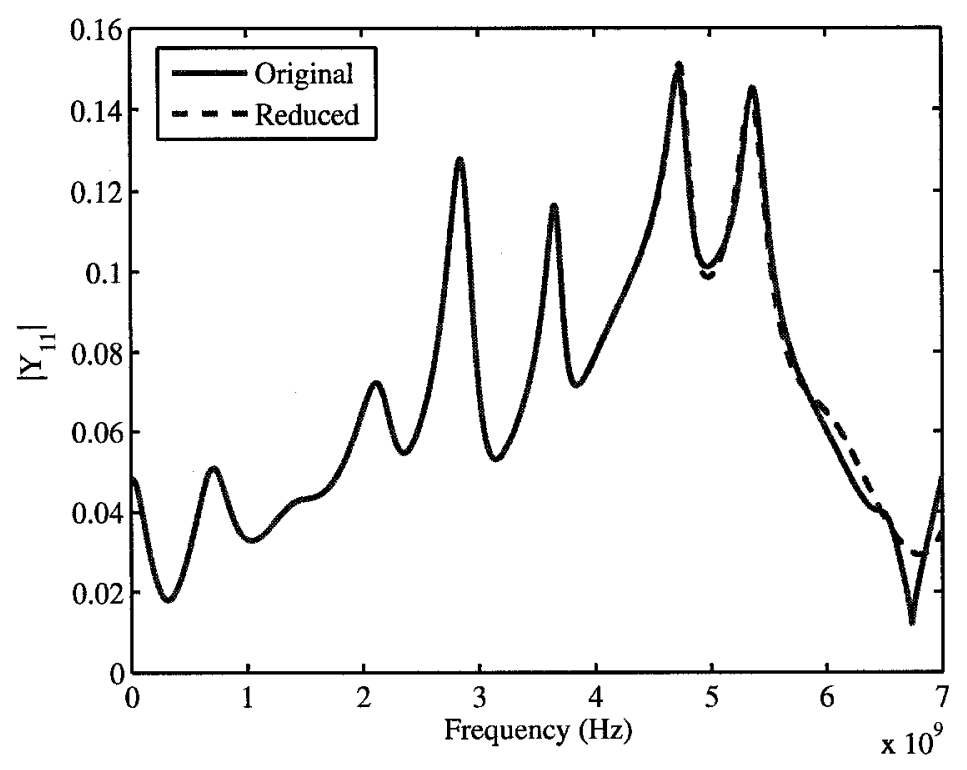

(a) Comparison of the system responses $Y_{11}$.

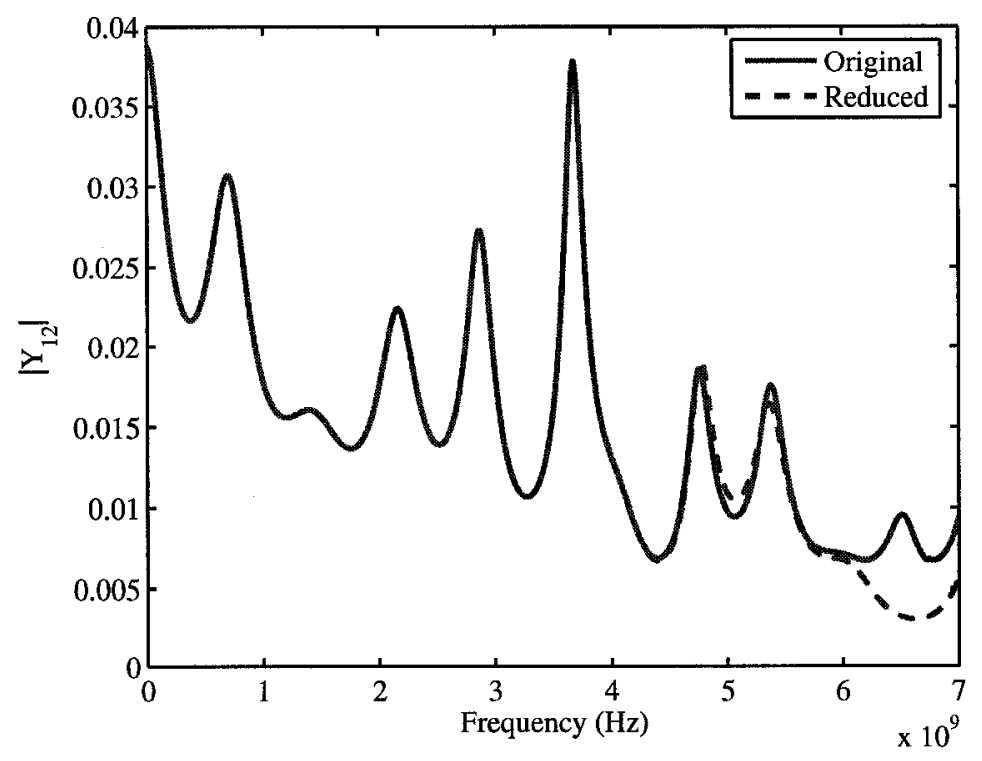

(b) Comparison of the system responses $Y_{12}$.

Figure 8.6: Frequency-domain responses (Example 3). 


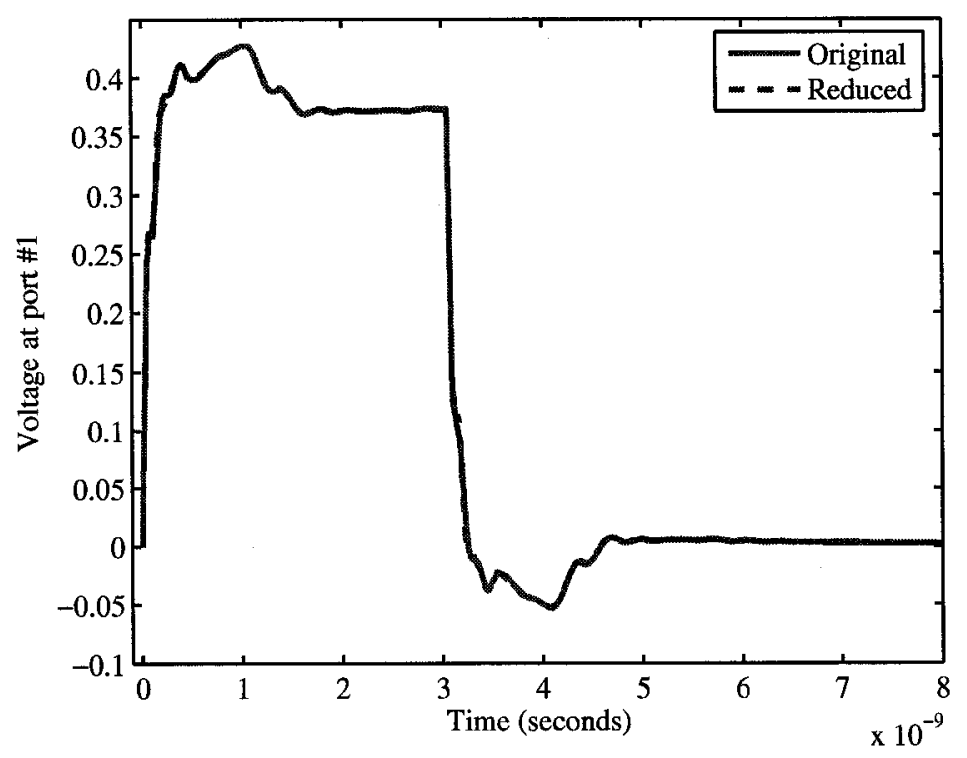

(a) Comparison of the transient responses at port 1.

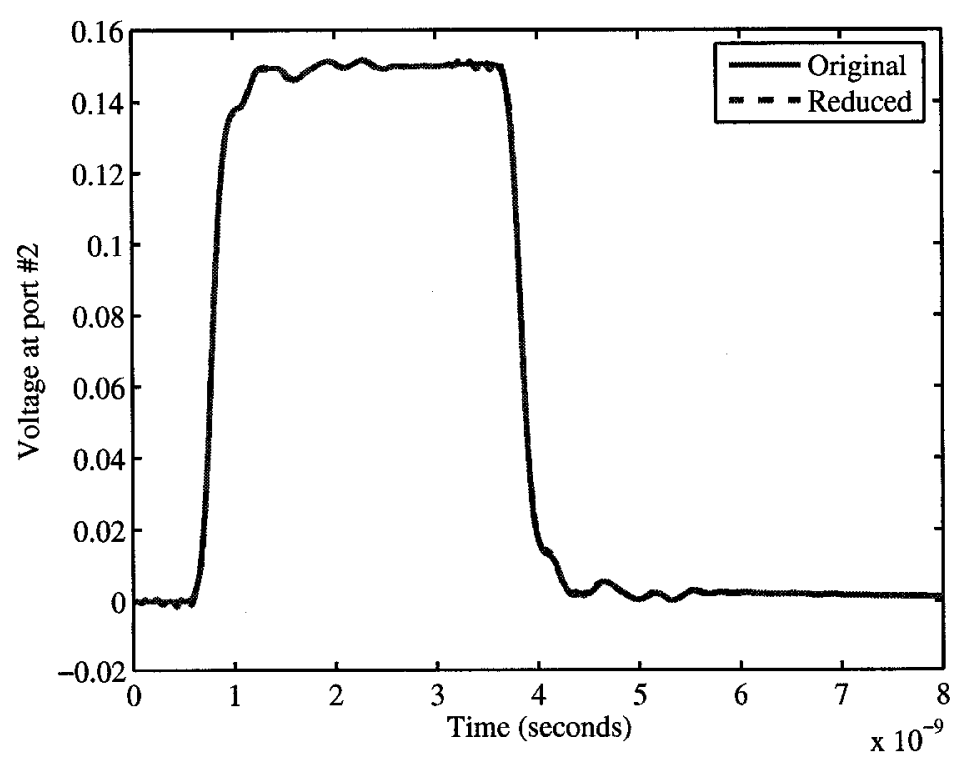

(b) Comparison of the transient responses at port 2.

Figure 8.7: Transient simulation results (Example 3). 
Table 8.1: CPU speedup (seconds / seconds).

\begin{tabular}{|c|c|}
\hline & Speedup \\
\hline \hline One-port Network & 14.67 \\
\hline Two-port Network & 16.51 \\
\hline
\end{tabular}

and the reduced-order system needs similar number of $\mathrm{L} / \mathrm{U}$ decomposition and F/B substitution due to the system structure preservation in the reduced system. However, for each L/U decomposition, $\frac{N^{3}}{3}-\frac{N}{3}$ and $\frac{n^{3}}{3}-\frac{n}{3}$ operations are required for the original system and the reduced-order system, respectively. And for each F/B substitution, $N^{2}$ and $n^{2}$ operations are required for the original system and the reduced system, respectively. Here $N$ denotes the dimension of the original system. $n$ denotes the dimension of the reduced system. Since $n$ is much smaller than $N$, there is a significant speedup in the numerical integration for the reduced system compared to the original system. 


\section{Chapter 9}

\section{Conclusions and Future Work}

This chapter presents a brief summary of the work that is presented in this thesis. In addition, future works are discussed.

\subsection{Summary}

Several novel techniques and algorithms have been presented in this thesis. The objectives set at the beginning of the thesis are fully achieved. The rest of this section summarize the developed techniques and algorithms.

A new theory is first presented to check the passivity of MoC-based macromodels. The proposed algebraic test for passivity, based on the developed theory, can verify the passivity of the $\mathrm{MoC}$ macromodel. If passivity violation occurs, the test can identify the exact locations at which this violation has occurred. Numerical experiments demonstrate that the locations of passivity violation are obtained accurately. Given that the MoC model is represented by a system of algebraic delay differential equations, the presented theory is a good candidate to tackle the issue of passivity of general dynamical systems described by ADDEs.

An efficient passivity enforcement algorithm is developed. The developed algorithm 
can correct the passivity violations for a non-passive MoC-based macromodel for a multiconductor transmission line. The resulting model can be embedded in a non-linear circuit simulator for fast signal integrity analysis. The passivity correction is achieved by compensating the negative eigenvalues of the real part of the system matrix via perturbing the residue matrix of the characteristic admittance matrix. The first-order perturbation theory is employed in the compensation. Numerical examples validate the high performance of the proposed algorithm.

An efficient circuit modeling approach is developed to model the frequency-dependent PUL parameters. The developed approach optimally approximates the FD-PUL parameters with rational functions with real poles. The PR-ness of the resulting rational functions can be easily enforced using passivity compensation techniques. A new network synthesis methodology based on Laplace elements, which is demonstrated to be more robust and efficient than the conventional lumped networks synthesis, is also proposed to transform the approximated rational functions into equivalent PUL networks, which can be adopted by many other modeling techniques.

A new algorithm, with two implementation methodologies, is developed to construct a reduced-order model for high-speed interconnects with FD-PUL parameters. The reducedorder model is created from TEs directly, without the intermediate step of approximating the TL with a large order lumped network. The developed algorithm features a robust numerical aspect. This feature comes as a result of constructing the orthonormal basis that spans the Hilbert subspace of the moments, without computing those moments explicitly. Numerical instability that results from numerical integration of the Telegrapher's equations has also been totally avoided through defining a weighted inner-product and norm mappings over the Hilbert space of the moments. This allows converting the Telegrapher's differential equations into differential algebraic equations. 
In addition, this thesis presents a new algorithm for performing model order reduction on large scale RLC circuits with delay elements. The proposed algorithm is validated by numerical experiments for RLC networks in the presence of lossless MTLs. A new formulation for the lossless MTL is proposed so that the passivity of the reduced-order system is guaranteed. The algorithm presented is useful for passive order reduction for a system represented by algebraic delay differential equations.

\subsection{Future work}

Some suggestions for the future research directions based on the developed techniques and algorithms presented in this thesis are discussed as follows.

1. The efficiency of the developed passivity verification algorithm for the method of characteristics based macromodels highly depends on the efficiency of solving the frequency-dependent generalized eigenvalue problems as stated in (4.10)-(4.11). The current solution is based on a two-step process. In the first step, we need to perform the frequency sweep for a common period to estimate the generalized eigenvalues. Developing a "frequency sweeping"-free approach is expected to increase the efficiency of the overall passivity verification algorithm.

2. The developed passivity enforcement algorithm is developed for the delay system constructed from method of characteristics based macromodels of multi-conductor transmission lines. It would be interesting if a general passivity enforcement algorithm can be developed for a general system described by algebraic delay differential equations.

3. In this thesis, integrated congruence transform has been demonstrated to be efficient in constructing reduced-order macromodels for uniform multi-conductor transmissin 
lines with frequency-dependent PUL parameters. The application of ICT to nonuniform MTLs with frequency-independnet PUL parameters has also been reported in the literature. However, the more important application of ICT on the general transmission lines with both FD-PUL parameters and non-uniform conductors at the same time is still an open topic.

4. A guaranteed passive model-order reduction technique for a general delay system is another excellent potential problem to be tackled in the future research activities. 


\section{Appendix A}

\section{Proof of Lemma 3.2.5}

It is required to prove, under the assumptions of Lemma 3.2 .5 , that $\tilde{Y}(s)$ satisfies the following three conditions,

1. $\tilde{\boldsymbol{Y}}(s)$ is analytic in $\Re(s)>0$;

2. $\tilde{\boldsymbol{Y}}\left(s^{\star}\right)=\tilde{\boldsymbol{Y}}^{\star}(s)$;

3. the third condition of PR-ness is equivalent to

$$
\boldsymbol{z}^{\star T}\left[\tilde{\boldsymbol{Y}}^{T}(-\jmath \omega)+\tilde{\boldsymbol{Y}}(\jmath \omega)\right] \boldsymbol{z} \geq 0 \quad \forall \omega \in \mathbb{R} \text { and } \forall \boldsymbol{z} \in \mathbb{C}
$$

It is straightforward observation to see that $\tilde{\boldsymbol{Y}}(s)$ satisfies the second condition by its definition. To show that $\tilde{\boldsymbol{Y}}(s)$ is analytic for $\Re(s)>0$, one needs to show that $\tilde{\boldsymbol{Y}}(s)$ satisfies Cauchy-Riemann equations $[49,50]$ with continuous partial derivatives in the right half plane. Proving that $\tilde{\boldsymbol{Y}}(s)$ satisfies C-R can be established straightforwardly by using Lemmas 2.2.1-2.2.4 and the fact that all of $\tilde{Y}_{c}(s), \tilde{P}(s)$ and $e^{s \tau}$ satisfy C-R and that $\frac{1}{1-e^{-2 s \tau} \tilde{P}^{2}(s)}$ does not have poles for $\Re(s)>0$. Since $\tilde{\boldsymbol{Y}}(s)$ has no singular points in $\Re(s)>0$ and has continuous partial derivatives, then this concludes that $\tilde{\boldsymbol{Y}}(s)$ is analytic for $\Re(s)>0$, 
and therefore satisfies the first condition of being PR. The rest of this appendix is dedicated to proving the last part of Lemma 3.2.5. More specifically, it is dedicated to prove that if $\left[\tilde{\boldsymbol{Y}}(s)+\tilde{\boldsymbol{Y}}^{\star}(s)\right]$ is non-negative definite on the $\jmath \omega$-axis $(\Re(s)=0)$ then it must be non-negative definite $\forall s \in \Re(s)>0$.

To this end, the basic argument of the minimum/maximum modulus theorem $[49,50]$. for a complex function is used. This theorem states that the minimum and maximum values for the real part of a given complex function on closed region, $\Omega$, in the complex-plane appears on its bounding contour $\mathcal{C}$ if this function is analytic throughout $\Omega$. For our purpose, we let $\Omega$ represent a semicircular area with a bounding contour $\mathcal{C}$ whose axis extends over the $j \omega$-axis and its center coincides with origin point of the complex-plan, while its circular arc lies in the right-half plane of the $s$-domain $(\Re(s)>0)$. It is obvious that as the radius of $\mathcal{C}$ becomes arbitrarily large $\Omega$ will extend to cover the right-half plane. Since $\tilde{\boldsymbol{Y}}(s)$ is analytic in $\Omega$ as proved earlier, then so is $\boldsymbol{z}^{\star T} \tilde{\boldsymbol{Y}}(s) \boldsymbol{z}$ for any vector $\boldsymbol{z}$. By the minimum/maximum modulus theorem, $\Re\left[\boldsymbol{z}^{\star T} \tilde{\boldsymbol{Y}}(s) \boldsymbol{z}\right]$ must have its minimum value occurring on $s \in \mathcal{C}$. Since $\boldsymbol{z}^{\star T} \tilde{\boldsymbol{Y}}(s) \boldsymbol{z}$ is a scalar which results in

$$
\Re\left[\boldsymbol{z}^{\star T} \tilde{\boldsymbol{Y}}(s) \boldsymbol{z}\right]=\frac{1}{2} \boldsymbol{z}^{\star T}\left(\tilde{\boldsymbol{Y}}^{T}\left(s^{\star}\right)+\tilde{\boldsymbol{Y}}(s)\right) \boldsymbol{z},
$$

then the minimum value for $\boldsymbol{z}^{\star T}\left(\tilde{\boldsymbol{Y}}^{T}\left(s^{\star}\right)+\tilde{\boldsymbol{Y}}(s)\right) \boldsymbol{z}$ in $s \in \Omega$ occurs only on $s \in \mathcal{C}$. Hence, if $\tilde{\boldsymbol{Y}}^{T}\left(s^{\star}\right)+\tilde{\boldsymbol{Y}}(s)$ is non-negative definite for $s \in \mathcal{C}$ then it must be non-negative definite in $s \in \Omega$. In other words, it is possible to check the non-negative definiteness in $\Omega$ via verification on the bounding contour $\mathcal{C}$. The rest of this appendix shows that as the radius of $\mathcal{C}$ approaches $\infty$, one only needs to check on that part of $\mathcal{C}$ that extends on the $\jmath \omega$-axis. 
Since $\tilde{P}(s)$ is a strict rational function, i.e., $\left.\tilde{P}(s)\right|_{s \rightarrow \infty}=0$, then

$$
\left.\tilde{\boldsymbol{Y}}(s)\right|_{s \rightarrow \infty}=\left[\begin{array}{cc}
\tilde{Y}_{c}(s) & 0 \\
0 & \tilde{Y}_{c}(s)
\end{array}\right]_{s \rightarrow \infty}
$$

It can therefore be deduced that if the radius of $\mathcal{C}$ approaches $\infty$, the behavior of $\tilde{\boldsymbol{Y}}(s)$ becomes asymptotically similar to that of $\tilde{Y}_{c}(s)$. Since $\tilde{Y}_{c}(s)$ is a stable rational function, its values at large values of $s$ becomes asymptotically independent of $\Re(s)$. Consequently,

$$
\left.\tilde{\boldsymbol{Y}}(s)\right|_{s \rightarrow \infty}=\left.\tilde{\boldsymbol{Y}}(s)\right|_{s \rightarrow j \infty}
$$

In other words, $\tilde{\boldsymbol{Y}}(s)$ on the circular arc of $\mathcal{C}$ approaches a limiting value which is equivalent to the limit value reached as $s \rightarrow \jmath \infty$. Given that the minimum value appears on $\mathcal{C}$, then $\boldsymbol{z}^{\star T}\left(\tilde{\boldsymbol{Y}}^{T}\left(s^{\star}\right)+\tilde{\boldsymbol{Y}}(s)\right) \boldsymbol{z} \geq 0 \quad \forall s \mid \Re(s)=0$ implies that $\boldsymbol{z}^{\star T}\left(\tilde{\boldsymbol{Y}}^{T}\left(s^{\star}\right)+\tilde{\boldsymbol{Y}}(s)\right) \boldsymbol{z} \geq$ $0 \forall s \mid \Re(s)>0$. This completes the proof of Lemma 3.2.5. 


\section{Appendix B}

\section{Proof of Proposition 3.3.1}

Based on MoC, the terminal description for the TL can be written in terms of the admittance matrix $\tilde{\boldsymbol{Y}}(s)$,

$$
\tilde{\boldsymbol{I}}(s)=\tilde{\boldsymbol{Y}}(s) \tilde{\boldsymbol{V}}(s)
$$

where $\tilde{\boldsymbol{I}}(s)=\left[\begin{array}{c}\tilde{I}_{1}(s) \\ \tilde{I}_{2}(s)\end{array}\right]$ and $\tilde{\boldsymbol{V}}(s)=\left[\begin{array}{c}\tilde{V}_{1}(s) \\ \tilde{V}_{2}(s)\end{array}\right]$ denote the approximated terminal currents and voltages of the transmission line, respectively, and $\tilde{\boldsymbol{Y}}(s)$ is defined as shown by (2.17). To prove Proposition 3.3.1, we assume that the "delay-free" matrix $P(s)$ and characteristic admittance $Y_{c}(s)$ are approximated by the following rational functions,

$$
\begin{gathered}
P(s) \approx \tilde{P}(s)=\frac{\sum_{i=0}^{n-1} a_{i} s^{i}}{\sum_{j=0}^{n} b_{j} s^{j}} \quad b_{0} \neq 0 \\
Y_{c}(s) \approx \tilde{Y}_{c}(s)=\frac{\sum_{i=0}^{q} r_{i} s^{i}}{\sum_{j=0}^{q} t_{j} s^{j}} \quad t_{0} \neq 0
\end{gathered}
$$

and introduce a set of auxiliary variables $\tilde{I}_{11}(s), I_{12}(s), I_{21}(s)$ and $\tilde{I}_{22}(s)$ defined as follows,

$$
\begin{array}{ll}
\tilde{I}_{11}(s)=\tilde{Y}_{11}(s) \tilde{V}_{1}(s), & I_{12}(s)=Y_{12}(s) \tilde{V}_{2}(s) \\
I_{21}(s)=Y_{21}(s) \tilde{V}_{1}(s), & \tilde{I}_{22}(s)=\tilde{Y}_{22}(s) \tilde{V}_{2}(s)
\end{array}
$$


where

$$
\begin{aligned}
& \tilde{Y}_{11}(s)=\tilde{Y}_{22}(s)=\frac{\phi(s)+e^{-2 s \tau} \vartheta(s)}{\alpha(s)-e^{-2 s \tau} \beta(s)} \\
& Y_{12}(s)=Y_{21}(s)=\frac{-2 e^{s \tau} \theta(s)}{\alpha(s)-e^{-2 s \tau} \beta(s)}
\end{aligned}
$$

and $\alpha(s), \beta(s), \theta(s), \phi(s)$ and $\vartheta(s)$ are polynomials in $s$ with coefficients, in respective order, $\alpha_{i}, \beta_{i}, \theta_{i}, \phi_{i}$ and $\vartheta_{i}$ given by (B.8)

$$
\begin{aligned}
\alpha_{i} & =\sum_{j=0}^{i} \sum_{k=0}^{j} b_{k} b_{j-k} t_{i-j} \\
\beta_{i} & =\sum_{j=0}^{i} \sum_{k=0}^{j} a_{k} a_{j-k} t_{i-j} \\
\theta_{i} & =\sum_{j=0}^{i} \sum_{k=0}^{j} a_{k} b_{j-k} r_{i-j} \\
\phi_{i} & =\sum_{j=0}^{i} \sum_{k=0}^{j} b_{k} b_{j-k}\left(r_{i-j}-\mu t_{i-j}\right) \\
\vartheta_{i} & =\sum_{j=0}^{i} \sum_{k=0}^{j} a_{k} a_{j-k}\left(r_{i-j}+\mu t_{i-j}\right)
\end{aligned}
$$

with $\mu$ being an arbitrary scalar value. The maximum order of the above polynomials are given by $\Delta=\max \{a, b+1, c+1, e+1, f+1\}$, where $a=2 n+q, b=2 n+q-2$, $c=2 n+q-1, e=\left\{2 n+q-1\right.$ if $\mu=\frac{r_{q}}{t_{q}}, 2 n+q$ otherwise $\}$, and $f=\left\{2 n+q-3\right.$ if $\mu=-\frac{r_{q}}{t_{q}}$, $2 n+q-2$ otherwise $\}$.

(B.4) and (B.5) can be realized in state space in form of the following set of ADDEs [100],

$$
\begin{aligned}
\mathcal{K}_{11} \dot{\boldsymbol{x}}(t) & =\mathcal{A}_{11} \boldsymbol{x}(t)+\mathcal{P}_{11} \boldsymbol{x}(t-2 \tau)+\mathcal{B}_{11} v_{1}(t) \\
\tilde{i}_{11}(t) & =\mathcal{C}_{11} \boldsymbol{x}(t)+\mathcal{Q}_{11} \boldsymbol{x}(t-2 \tau) \\
\mathcal{K}_{12} \dot{\boldsymbol{z}}(t) & =\mathcal{A}_{12} \boldsymbol{z}(t)+\mathcal{P}_{12} \boldsymbol{z}(t-2 \tau)+\mathcal{B}_{12} v_{2}(t) \\
i_{12}(t) & =\mathcal{Q}_{12} \boldsymbol{z}(t-\tau) \\
\mathcal{K}_{11} \dot{\hat{\boldsymbol{x}}}(t) & =\mathcal{A}_{11} \hat{\boldsymbol{x}}(t)+\mathcal{P}_{11} \hat{\boldsymbol{x}}(t-2 \tau)+\mathcal{B}_{11} v_{1}(t) \\
i_{21}(t) & =\mathcal{Q}_{12} \hat{\boldsymbol{x}}(t-\tau) \\
\mathcal{K}_{12} \dot{\boldsymbol{z}}(t) & =\mathcal{A}_{12} \hat{\boldsymbol{z}}(t)+\mathcal{P}_{12} \hat{\boldsymbol{z}}(t-2 \tau)+\mathcal{B}_{12} v_{2}(t) \\
\tilde{i}_{22}(t) & =\mathcal{C}_{11} \hat{\boldsymbol{z}}(t)+\mathcal{Q}_{11} \hat{\boldsymbol{z}}(t-2 \tau)
\end{aligned}
$$


where

$$
\begin{aligned}
& \mathcal{K}_{11}=\mathcal{K}_{12}=\left[\begin{array}{cccc}
1 & 0 & \ldots & 0 \\
\vdots & & \ddots & \vdots \\
0 & 0 & 1 & 0 \\
0 & 0 & \ldots & \alpha_{\Delta}
\end{array}\right] \\
& \mathcal{A}_{11}=\mathcal{A}_{12}=\left[\begin{array}{cccc}
0 & 1 & \ldots & 0 \\
\vdots & \vdots & \ddots & \vdots \\
0 & 0 & \ldots & 1 \\
-\alpha_{0} & -\alpha_{1} & \ldots & -\alpha_{\Delta-1}
\end{array}\right] \\
& \mathcal{P}_{11}=\mathcal{P}_{12}=\left[\begin{array}{cccc}
0 & 0 & \ldots & 0 \\
\vdots & \vdots & \vdots & \vdots \\
0 & 0 & \ldots & 0 \\
\beta_{0} & \beta_{1} & \ldots & \beta_{\Delta-1}
\end{array}\right] \\
& \mathcal{B}_{11}=\mathcal{B}_{12}=\left[\begin{array}{llll}
0 & \cdots & 0 & 1
\end{array}\right]^{T} \\
& \mathcal{C}_{11}=\left[\begin{array}{llll}
\phi_{0} & \phi_{1} & \ldots & \phi_{\Delta-1}
\end{array}\right] \\
& \mathcal{Q}_{11}=\left[\begin{array}{llll}
\vartheta_{0} & \vartheta_{1} & \ldots & \vartheta_{\Delta-1}
\end{array}\right] \\
& \mathcal{Q}_{12}=\left[\begin{array}{llll}
-2 \theta_{0} & -2 \theta_{1} & \ldots & -2 \theta_{\Delta-1}
\end{array}\right] \\
& \boldsymbol{x}(t)=\left[\begin{array}{lll}
x_{1}(t) & \ldots & x_{\Delta}(t)
\end{array}\right]^{T} \\
& \boldsymbol{z}(t)=\left[\begin{array}{lll}
z_{1}(t) & \ldots & z_{\Delta}(t)
\end{array}\right]^{T} \\
& \hat{\boldsymbol{x}}(t)=\left[\begin{array}{lll}
\hat{x}_{1}(t) & \ldots & \hat{x}_{\Delta}(t)
\end{array}\right]^{T} \\
& \hat{\boldsymbol{z}}(t)=\left[\begin{array}{lll}
\hat{z}_{1}(t) & \ldots & \hat{z}_{\Delta}(t)
\end{array}\right]^{T}
\end{aligned}
$$

Combining the systems of ADDEs in (B.9)-(B.12) and the fact that

$$
\left\{\begin{array}{l}
\tilde{I}_{1}(s)=\tilde{I}_{11}(s)+\mu \tilde{V}_{1}(s)+I_{12}(s) \\
\tilde{I}_{2}(s)=I_{21}(s)+\mu \tilde{V}_{2}(s)+\tilde{I}_{22}(s)
\end{array},\right.
$$

we can derive the relation between the terminal voltages $\tilde{\boldsymbol{v}}(t)$ and currents $\tilde{\boldsymbol{i}}(t)$ in an unified system of ADDEs,

$$
\left\{\begin{aligned}
\mathcal{K} \dot{\boldsymbol{\chi}}(t) & =\mathcal{A} \boldsymbol{\chi}(t)+\mathcal{P} \boldsymbol{\chi}(t-2 \tau)+\mathcal{B} \boldsymbol{v}(t) \\
\tilde{\boldsymbol{i}}(t) & =\mathcal{C}_{\boldsymbol{\chi}}(t)+\mathcal{Q}_{1} \boldsymbol{\chi}(t-\tau)+\mathcal{Q}_{2} \boldsymbol{\chi}(t-2 \tau)+\mathcal{D} \boldsymbol{v}(t)
\end{aligned}\right.
$$


where

$$
\begin{aligned}
\mathcal{K}=\left[\begin{array}{cc}
\mathcal{K}_{11} & 0 \\
0 & \mathcal{K}_{12}
\end{array}\right], & \mathcal{A}=\left[\begin{array}{cc}
\mathcal{A}_{11} & 0 \\
0 & \mathcal{A}_{12}
\end{array}\right], \\
\mathcal{P}=\left[\begin{array}{cc}
\mathcal{P}_{11} & 0 \\
0 & \mathcal{P}_{12}
\end{array}\right], & \mathcal{B}=\left[\begin{array}{cc}
\mathcal{B}_{11} & 0 \\
0 & \mathcal{B}_{12}
\end{array}\right] \\
\mathcal{C}=\left[\begin{array}{cc}
\mathcal{C}_{11} & 0 \\
0 & \mathcal{C}_{11}
\end{array}\right], & \mathcal{Q}_{1}=\left[\begin{array}{cc}
0 & \mathcal{Q}_{12} \\
\mathcal{Q}_{12} & 0
\end{array}\right], \\
\mathcal{Q}_{2}=\left[\begin{array}{cc}
\mathcal{Q}_{11} & 0 \\
0 & \mathcal{Q}_{11}
\end{array}\right], & \mathcal{D}=\left[\begin{array}{cc}
\mu & 0 \\
0 & \mu
\end{array}\right] \\
\boldsymbol{\chi}(t)=\left[\boldsymbol{x}(t)^{T} \boldsymbol{z}(t)^{T}\right]^{T} &
\end{aligned}
$$




\section{Appendix C}

\section{Proof of Propositions 4.3.2 and 4.3.3}

This appendix is dedicated to present the proof of Proposition 4.3.2. The proof for Proposition 4.3.3 follows at the end. By introducing new variables $\Pi_{1}(s), \Pi_{2}(s)$ and $\Pi_{3}(s),(4.2)$ can be redefined to be,

$$
\boldsymbol{Y}_{1}(s)=\boldsymbol{\Pi}_{1}^{-1}(s) \boldsymbol{\Pi}_{2}(s) \Pi_{3}(s)
$$

where,

$$
\begin{aligned}
& \boldsymbol{\Pi}_{1}(s)=\boldsymbol{I}_{m}+\boldsymbol{M} e^{-s \tau} \tilde{\boldsymbol{P}}(s) \boldsymbol{M}^{-1} \\
& \boldsymbol{\Pi}_{2}(s)=\boldsymbol{I}_{m}-\boldsymbol{M} e^{-s \tau} \tilde{\boldsymbol{P}}(s) \boldsymbol{M}^{-1} \\
& \boldsymbol{\Pi}_{3}(s)=\tilde{\boldsymbol{Y}}_{c}(s)
\end{aligned}
$$

The goal in the rest of the proof will be looking for two dynamical systems with delays $\left(\Sigma_{1}\right.$ and $\left.\Sigma_{2}\right)$ and one linear time invariant system $\left(\Sigma_{3}\right)$ such that they have transfer functions equal to $\Pi_{1}(s), \boldsymbol{\Pi}_{2}(s)$ and $\Pi_{3}(s)$, respectively. We rewrite $\tilde{\boldsymbol{P}}(s)$ and $\tilde{\boldsymbol{Y}}_{c}(s)$ in the 
following form:

$$
\begin{aligned}
\tilde{\boldsymbol{P}}(s) & =\left[P_{i j}(s)\right]_{m \times m}=\left[\sum_{k=1}^{N_{p}} \frac{c_{k}^{(i, j)}}{s-a_{k}}\right]_{m \times m} \\
\tilde{\boldsymbol{Y}}_{\boldsymbol{c}}(s) & =\left[Y_{c i j}(s)\right]_{m \times m}=\left[r_{0}^{(i, j)}+\sum_{k=1}^{N_{y}} \frac{r_{k}^{(i, j)}}{s-p_{k}}\right]_{m \times m}
\end{aligned}
$$

where $c_{k}^{(i, j)}, a_{k}, r_{0}^{(i, j)}, r_{k}^{(i, j)}, p_{k}$ are real constants used in representing $\tilde{\boldsymbol{P}}(s)$ and $\tilde{\boldsymbol{Y}}_{\boldsymbol{c}}(s)$ matrices, with $N_{p}$ and $N_{y}$ being the number of required poles. Substituting (C.5) and $\tau$ into (C.2), after some manipulations, yields,

$$
\boldsymbol{\Pi}_{1}(s)=\boldsymbol{I}_{m}+\boldsymbol{M}\left[\sum_{k=1}^{N_{p}} \frac{c_{k}^{(i, j)}}{s-a_{k}} e^{-s \tau_{i}}\right] \boldsymbol{M}^{-1}
$$

Let $\mathcal{A}_{p}, \mathcal{B}_{p}$ and $\mathcal{C}_{p}^{(i, j)}$ be the state-space minimal realization of $\sum_{k=1}^{N_{p}} \frac{c_{k}^{(i, j)}}{s-a_{k}}$. (Jordan Canonical form is used in our implementation. For details on how to transfer rational functions to state-space representation, please refer to [100]). The dynamical system with delay representation for $\Pi_{1}(s)$ can then be obtained as:

$$
\Sigma_{1}:\left\{\begin{aligned}
\mathcal{K}_{1} \dot{\boldsymbol{\chi}}(t) & =\mathcal{A}_{1} \boldsymbol{\chi}(t)+\mathcal{B}_{1} \boldsymbol{u}_{1}(t) \\
\boldsymbol{y}_{1}(t) & =\sum_{k=1}^{m} \mathcal{C}_{1}^{k} \chi\left(t-\tau_{k}\right)+\mathcal{D}_{1} \boldsymbol{u}_{1}(t)
\end{aligned}\right.
$$

where $\mathcal{K}_{1}$ and $\mathcal{D}_{1}$ are identity matrices with appropriate size. $\mathcal{A}_{1}$ is a $m \times m$ diagonal block matrix in the form of $\mathcal{A}_{1}=\operatorname{diag}\left\{\mathcal{A}_{p}, \ldots, \mathcal{A}_{p}\right\} . \mathcal{B}_{1}$ and $\mathcal{C}_{1}^{k}$ are defined as follows,

$$
\begin{aligned}
\mathcal{B}_{1} & =\operatorname{diag}\left\{\mathcal{B}_{p}, \ldots, \mathcal{B}_{p}\right\} M^{-1} \\
\mathcal{C}_{1}^{k} & =\boldsymbol{M C}^{k}
\end{aligned}
$$

and $\mathcal{C}^{k}$ is a $m \times m$ block matrix with only the $k^{\text {th }}$ row being non-zero blocks given by $\left[\mathcal{C}_{p}^{(k, 1)}, \ldots, \mathcal{C}_{p}^{(k, m)}\right]$. Note that $\mathcal{K}_{1}$ is explicitly kept in the system representation to emphasize that the theory developed in this paper can be applied to general algebraic systems.

Similarly, the dynamical system with delays representation for $\Pi_{2}(s)$ can be obtained 
as:

$$
\Sigma_{2}:\left\{\begin{aligned}
\mathcal{K}_{2} \dot{\psi}(t) & =\mathcal{A}_{2} \boldsymbol{\psi}(t)+\mathcal{B}_{2} \boldsymbol{u}_{2}(t) \\
\boldsymbol{u}_{1}(t) & =\sum_{k=1}^{m} \mathcal{C}_{2}^{k} \boldsymbol{\psi}\left(t-\tau_{k}\right)+\mathcal{D}_{2} \boldsymbol{u}_{2}(t)
\end{aligned}\right.
$$

where,

$$
\mathcal{K}_{1}=\mathcal{K}_{2}, \quad \mathcal{A}_{1}=\mathcal{A}_{2}, \quad \mathcal{B}_{1}=\mathcal{B}_{2}, \quad \mathcal{D}_{1}=\mathcal{D}_{2}, \quad-\mathcal{C}_{1}^{k}=\mathcal{C}_{2}^{k}
$$

$\Pi_{3}(s)$ is a regular matrix rational function [100], and hence it can be realized as,

$$
\Sigma_{3}:\left\{\begin{aligned}
\mathcal{K}_{3} \dot{\boldsymbol{Z}}(t) & =\mathcal{A}_{3} \boldsymbol{Z}(t)+\mathcal{B}_{3} \boldsymbol{u}_{3}(t) \\
\boldsymbol{u}_{2}(t) & =\mathcal{C}_{3} \boldsymbol{Z}(t)+\mathcal{D}_{3} \boldsymbol{u}_{3}(t)
\end{aligned}\right.
$$

where matrices $\mathcal{K}_{3}, \mathcal{A}_{3}, \mathcal{B}_{3}, \mathcal{C}_{3}$ and $\mathcal{D}_{3}$ are given in terms of the coefficients from the rational function of $\tilde{\boldsymbol{Y}}_{c}(s)$.

Further, it is clear that the transfer functions of $\Sigma_{1}, \Sigma_{2}$ and $\Sigma_{3}$ are equal to $\Pi_{1}(s), \Pi_{2}(s)$ and $\Pi_{3}(s)$, respectively, and defined to be,

$$
\begin{aligned}
& \Pi_{1}(s)=\mathcal{C}_{1}(s)\left(s \mathcal{K}_{1}-\mathcal{A}_{1}\right)^{-1} \mathcal{B}_{1}+\mathcal{D}_{1} \\
& \Pi_{2}(s)=\mathcal{C}_{2}(s)\left(s \mathcal{K}_{2}-\mathcal{A}_{2}\right)^{-1} \mathcal{B}_{2}+\mathcal{D}_{2} \\
& \Pi_{3}(s)=\mathcal{C}_{3}\left(s \mathcal{K}_{3}-\mathcal{A}_{3}\right)^{-1} \mathcal{B}_{3}+\mathcal{D}_{3}
\end{aligned}
$$

where

$$
\mathcal{C}_{1}(s)=-\mathcal{C}_{2}(s)=\sum_{k=1}^{m} \mathcal{C}_{1}^{k} e^{-s \tau_{k}}
$$

This completes the proof of Proposition 4.3.2.

It is clear through observing (4.2) and (4.3) that $\boldsymbol{Y}_{1}(s)$ and $\boldsymbol{Y}_{2}(s)$ possess similar structures except for having $-\mathcal{C}_{1}^{k}$ instead of $\mathcal{C}_{1}^{k}$. Similar steps as above can then be carried out 
to prove Proposition 4.3.3. 


\section{Appendix D}

\section{Numerical Allocation of Passivity Violation}

This appendix presents a numerical procedure used to locate the passivity violation regions of a non-passive MoC-based macromodel. This procedure is an extension of an earlier technique presented in Chapter 3 to handle the special case of a two-conductor TL macromodel.

Similar to Chapter 3, the proposed procedure is carried out in two steps. In the first step, a rough estimate of those intervals on the $\jmath \omega$-axis, where passivity violation is likely to occur, is obtained. We denote such a generic interval by two delimiting points $\left[\jmath \omega_{1}, \jmath \omega_{2}\right]$. The second step aims at identifying passivity violation points more accurately, and is carried out by replacing every exponential term in (4.28)-(4.29) with an approximate Chebyshev polynomial within the interval $\left[j \omega_{1}, \jmath \omega_{2}\right]$. This substitution transforms the FD-GEP problem as in (4.10) and (4.11) into two polynomial eigenvalue problems as described by the following lemma.

Lemma D.0.10 Let each exponential function in (4.28) (or (4.29)) be approximated by a 
q-th order Chebyshev polynomial of the first kind, for the interval of $\left[\jmath \omega_{1}, \jmath \omega_{2}\right]$, i.e.,

$$
\begin{aligned}
e^{-s \tau_{k}} & \approx \sum_{h=0}^{q} t_{h}^{\left(\hat{W}^{(1)}, k\right)} T_{h}(\hat{s}) \\
e^{s \tau_{k}} & \approx \sum_{h=0}^{q} t_{h}^{\left(\bar{W}^{(1)}, k\right)} T_{h}(\hat{s}) \\
e^{s\left(\tau_{k}-\tau_{l}\right)} & \approx \sum_{h=0}^{q} t_{h}^{\left(W^{(1)}, k, l\right)} T_{h}(\hat{s})
\end{aligned}
$$

where $k, l \in\{1, \ldots, m\}, t_{h}^{(\ldots)}$ are coefficients of the Chebyshev polynomials, and

$$
\hat{s}=2 \frac{s-\jmath \omega_{1}}{\jmath \omega_{2}-\jmath \omega_{1}}-1 \in[-1,1]
$$

Then values of $s \in\left[\jmath \omega_{1}, \jmath \omega_{2}\right]$ satisfying the FD-GEP in (4.10) (or (4.11)) can be approximated from,

$$
s=\frac{\jmath \omega_{2}-\jmath \omega_{1}}{2} \hat{s}+\frac{\jmath \omega_{2}+\jmath \omega_{1}}{2}
$$

where $\hat{s}$ is the solution of the following generalized polynomial eigenvalue problem,

$$
\left(\sum_{i=0}^{q} \boldsymbol{\Upsilon}_{i} \hat{s}^{i}\right) \boldsymbol{\xi}=\mathbf{0}
$$

where $\Upsilon_{i}$ is defined in the following proof.

Proof The following paragraphs show the proof for the case of (4.10). The proof for (4.11) is very similar and omitted for brevity. It is straightforward to conclude that (D.5) is true from (D.4). Hence, the only thing we need to prove is that $\hat{s}$ satisfies the GPEP in (D.6). To proceed with the proof, we substitute (D.1)-(D.3) and (D.5) into (4.10) to obtain,

$$
\left(\sum_{h=0}^{q} \boldsymbol{\Psi}_{h} T_{h}(\hat{s})\right) \boldsymbol{\xi}=\mathbf{0}
$$


where,

$$
\begin{aligned}
& \boldsymbol{\Psi}_{h}=\sum_{k=1}^{m}\left(\hat{\boldsymbol{W}}_{k}^{(1)} t_{h}^{\left(\hat{W}^{(1)}, k\right)}+\overline{\boldsymbol{W}}_{k}^{(1)} t_{h}^{\left(\bar{W}^{(1)}, k\right)}\right)+\sum_{k, l=1}^{m} \boldsymbol{W}_{k, l}^{(1)} t_{h}^{\left(W^{(1)}, k, l\right)}+\boldsymbol{\Xi}_{h} \\
& \boldsymbol{\Xi}_{h}= \begin{cases}\mathcal{V}^{(1)}-\frac{\jmath \omega_{2}+\jmath \omega_{1}}{2} \boldsymbol{K}_{1} & i=0 \\
-\frac{\jmath \omega_{2}-\jmath \omega_{1}}{2} \boldsymbol{K}_{1} & i=1 \\
\mathbf{0} & i \geq 2\end{cases}
\end{aligned}
$$

The left-side of (D.7) can be further transformed into the following polynomial eigenvalue problem in $\hat{s}$ by substituting each Chebyshev function $T_{h}(\hat{s})$ with its associated regular polynomial, i.e., $T_{h}(\hat{s})=\sum_{i=0}^{q} b_{h}^{i} \hat{s}^{i}$, where $b_{h}^{i}$ 's are constant coefficients shown in [89].

$$
\left(\sum_{i=0}^{q} \Upsilon_{i} \hat{s}^{i}\right) \boldsymbol{\xi}=\mathbf{0}
$$

where

$$
\Upsilon_{i}=\sum_{h=0}^{q} \Psi_{h} b_{h}^{i}
$$

Lemma D.0.10 indicates that if there exists a purely imaginary value for $s$, say $s=\jmath \omega_{o}$, that satisfies the GPEP specified by (D.5)-(D.6) and if $\jmath \omega_{0}$ lies within the frequency interval, $\left[\jmath \omega_{1}, \jmath \omega_{2}\right]$, then $s$ is a purely imaginary solution of the FD-GEP in (4.10). Repeating the approximation for all the frequency intervals (obtained from the first step), and solving (D.5)-(D.6) for imaginary $s$ in each approximation, enable finding all the imaginary solutions to the FD-GEP in (4.10). 


\section{Appendix E}

\section{Alternative Implementation Methodology for ICT}

\section{E.1 Introduction}

In Chapter 7, a new algorithm that extends the idea of integrated congruence transform, has been presented to construct the reduced-order macromodel for the high-speed interconnects with frequency-dependent PUL parameters. The proposed algorithm proceeds in the following steps: modeling the FD-PUL matrices with PR matrix rational functions, which are then realized in the form of lumped networks; writing the MNA formulation for the lumped networks and reformulating the classical TEs by generalized TEs based on the MNA formulation; and constructing the reduced-order model via applying the typical ICT reduction framework on the general TEs, which are in the form of DAEs. The new algorithm provides a new approach to perform implicit moment matching on a system described by a mixed set of linear differential algebraic equations. However, the passivity of the resulting reduced model is guaranteed only if $\mathcal{G}_{Z}, \mathcal{C}_{Z}, \mathcal{G}_{Y}$ and $\mathcal{C}_{Y}$ from the MNA formulation satisfy the specific conditions stated in Chapter 7 , which requires that all the components in the lumped circuit model of PUL matrices are positive. However, this is a challenging problem 
as the task of realizing positive-real matrix functions in terms of all positive lumped circuit elements can be difficult [118]. The presence of negative components in the lumped model may make the governing matrices of the ICT formulation to be nonconforming to the criteria required for guaranteed passive reduction. As a result, the reduced-order models generated by ICT may become non-passive. To address this problem, an alternative methodology is developed in this appendix, to directly incorporate state-space equations corresponding to the positive-real rational functions representing the frequency-dependence of PUL parameters, into Telegrapher's equations. The proposed formulation overcomes the need for explicit lumped circuit realization with all positive elements from matrix PR rational functions. Based on the new formulation of TEs, the ICT technique presented in Chapter 7 can still be applied to construct guaranteed passive reduced model. The numerical examples show that, the new method while providing robustness to the passive macromodeling process, also yields computationally superior results.

The rest of this appendix is organized as follows [37]. Section E.2 presents a new formulation of general TEs, incorporating the PUL impedance and admittance. The reduced model is then obtained in the same framework as introduced in Chapter 7. Section E.3 demonstrates the passivity preservation in the reduced model based on the proposed formulation. Section E.4 discusses the computational complexity in the process of reducedorder model construction. Numerical results are presented in Section E.5 to validate the performance of the proposed TEs formulation.

\section{E.2 Proposed formulation}

For the purpose of presentation, the PR rational function approximation of PUL impedance $\mathcal{Z}(s)=\boldsymbol{R}(s)+s \boldsymbol{L}(s)$ and admittance $\mathcal{Y}(s)=\boldsymbol{G}(s)+s \boldsymbol{C}(s)$ are assumed to have been 
obtained $[59,61]$ in the form

$$
\begin{aligned}
\tilde{\mathcal{Z}}(s) & =\underbrace{\sum_{i=1}^{N_{z}} \frac{\mathbb{K}_{z i}}{s-p_{z i}}+\mathbb{D}_{z}}_{\overline{\mathcal{Z}}(s)}+s \mathbb{E}_{z} \\
\tilde{\mathcal{Y}}(s) & =\underbrace{\sum_{i=1}^{N_{y}} \frac{\mathbb{K}_{y_{i}}}{s-p_{y_{i}}}+\mathbb{D}_{y}}_{\overline{\mathcal{Y}}(s)}+s \mathbb{E}_{y}
\end{aligned}
$$

such that, $\mathbb{D}_{z}, \mathbb{E}_{z}, \mathbb{D}_{y}$ and $\mathbb{E}_{y}$ are symmetric non-negative definite matrices; and $\overline{\mathcal{Z}}(s)$ and $\overline{\mathcal{Y}}(s)$ are positive-real matrix rational functions. Here, $\mathbb{K}_{z}$ and $\mathbb{K}_{y}$ are the residue matrices, $p_{z_{i}}$ and $p_{y_{i}}$ are the poles; and $N_{z}$ and $N_{y}$ are the number of poles for $\overline{\mathcal{Z}}(s)$ and $\overline{\mathcal{Y}}(s)$, respectively.

Let $\overline{\mathcal{Z}}(s)$ and $\overline{\mathcal{Y}}(s)$ be represented in the state-space form as:

$$
\begin{aligned}
& \overline{\mathcal{Z}}(s)=\boldsymbol{C}_{z}\left(s \boldsymbol{I}-\boldsymbol{A}_{z}\right)^{-1} \boldsymbol{B}_{z}+\mathbb{D}_{z} \\
& \overline{\mathcal{Y}}(s)=\boldsymbol{C}_{y}\left(s \boldsymbol{I}-\boldsymbol{A}_{y}\right)^{-1} \boldsymbol{B}_{y}+\mathbb{D}_{y}
\end{aligned}
$$

where, matrices $\boldsymbol{A}_{z}, \boldsymbol{B}_{z}, \boldsymbol{C}_{z}, \boldsymbol{A}_{y}, \boldsymbol{B}_{y}$ and $\boldsymbol{C}_{y}$ are composed of coefficients used to describe the rational functions of $\overline{\mathcal{Z}}(s)$ and $\overline{\mathcal{Y}}(s)$ in (E.1)-(E.2). There are several ways to obtain these matrices. Readers are referred to [1] for details. It is shown that directly incorporating (E.3)-(E.4) into TEs in (2.1), the corresponding ICT may not be guaranteed passive. This was handled in Chapter 7 by realizing (E.1)-(E.2) by lumped circuit realization with all positive elements. However, this can be a challenging task in the case of matrix PR functions. To avoid this difficulty, an alternative approach is presented below.

Let $\boldsymbol{Q}_{z}=Q_{z}^{T} \geq 0$ and $Q_{y}=Q_{y}^{T} \geq 0$ be the solutions of the following two linear 
matrix inequalities, respectively,

$$
\begin{gathered}
{\left[\begin{array}{cc}
-Q_{z} A_{z}-A_{z}^{T} Q_{z} & C_{z}^{T}-Q_{z} B_{z} \\
C_{z}-B_{z}^{T} Q_{z} & \mathbb{D}_{z}+\mathbb{D}_{z}^{T}
\end{array}\right] \geq 0} \\
{\left[\begin{array}{cc}
-Q_{y} A_{y}-A_{y}^{T} Q_{y} & C_{y}^{T}-Q_{y} B_{y} \\
C_{y}-B_{y}^{T} Q_{y} & \mathbb{D}_{y}+\mathbb{D}_{y}^{T}
\end{array}\right] \geq 0}
\end{gathered}
$$

According to positive-real lemma [65], the above solutions for $\boldsymbol{Q}_{z}$ and $\boldsymbol{Q}_{y}$ exist if the systems (E.3) and (E.4) are passive. Efficient methods for the computation of $\boldsymbol{Q}_{z}$ and $\boldsymbol{Q}_{y}$ satisfying (E.5) and (E.6), respectively, can be found in [119].

Next, introducing the set of auxiliary variables, $\boldsymbol{V}_{Z}(x, s)$ and $\boldsymbol{I}_{Y}(x, s)$, defined as follows

$$
\begin{aligned}
\left(s \boldsymbol{Q}_{z}-\boldsymbol{Q}_{z} \boldsymbol{A}_{z}\right) \boldsymbol{V}_{Z}(x, s) & =\boldsymbol{Q}_{z} \boldsymbol{B}_{z} \boldsymbol{I}(x, s) \\
\left(s \boldsymbol{Q}_{y}-\boldsymbol{Q}_{y} \boldsymbol{A}_{y}\right) \boldsymbol{I}_{Y}(x, s) & =\boldsymbol{Q}_{y} \boldsymbol{B}_{y} \boldsymbol{V}(x, s)
\end{aligned}
$$

and substituting (E.1)-(E.8) into (2.1) we get,

$$
\tilde{\boldsymbol{T}} \frac{\partial}{\partial x} \tilde{\boldsymbol{X}}(x, s)=-(\tilde{\mathcal{N}}+s \tilde{\mathcal{M}}) \tilde{\boldsymbol{X}}(x, s)
$$

where,

$$
\begin{aligned}
& \tilde{T}=\left[\begin{array}{cccc}
0 & \boldsymbol{I}_{m} & 0 & 0 \\
\boldsymbol{I}_{m} & 0 & 0 & 0 \\
0 & 0 & 0 & 0 \\
0 & 0 & 0 & 0
\end{array}\right], \tilde{\mathcal{M}}=\left[\begin{array}{cccc}
\mathbb{E}_{z} & 0 & 0 & 0 \\
0 & \mathbb{E}_{y} & 0 & 0 \\
0 & 0 & Q_{z} & 0 \\
0 & 0 & 0 & Q_{y}
\end{array}\right] \\
& \tilde{\mathcal{N}}=\left[\begin{array}{cccc}
\mathbb{D}_{z} & 0 & C_{z} & 0 \\
0 & \mathbb{D}_{y} & 0 & C_{y} \\
-\boldsymbol{Q}_{z} \boldsymbol{B}_{z} & \mathbf{0} & -\boldsymbol{Q}_{z} \boldsymbol{A}_{z} & \mathbf{0} \\
\mathbf{0} & -\boldsymbol{Q}_{y} \boldsymbol{B}_{y} & \mathbf{0} & -\boldsymbol{Q}_{y} \boldsymbol{A}_{y}
\end{array}\right], \tilde{\boldsymbol{X}}(x, s)=\left[\begin{array}{c}
\boldsymbol{I}(x, s) \\
\boldsymbol{V}(x, s) \\
\boldsymbol{V}_{Z}(x, s) \\
\boldsymbol{I}_{Y}(x, s)
\end{array}\right]
\end{aligned}
$$

It is important to highlight that the new formulation in (E.9) is significantly different from 
the formulation in Chapter 7 in that it permits the direct incorporation of FD-PUL statespace matrices in Telegrapher's equations in a form suitable for the application of passive ICT algorithm. Next, the reduced-order model for (E.9) can be constructed after following the procedures presented in Chapter 7 ,

$$
\begin{aligned}
(\hat{\overline{\mathcal{N}}}+s \hat{\overline{\mathcal{M}}}) \hat{\boldsymbol{X}}_{\mathcal{Q}}(s) & =\hat{\overline{\boldsymbol{b}}} \tilde{\boldsymbol{V}}(s) \\
\tilde{\boldsymbol{I}}(s) & =\hat{\overline{\boldsymbol{b}}}^{T} \hat{\boldsymbol{X}}_{\mathcal{Q}}(s)
\end{aligned}
$$

where, $\hat{\overline{\mathcal{N}}}, \hat{\mathcal{\mathcal { M }}}, \hat{\overline{\boldsymbol{b}}}$ and $\hat{\boldsymbol{X}}_{\mathcal{Q}}(s)$ are the same as defined in Chapter 7, except replacing the definitions of $\tilde{\boldsymbol{T}}, \tilde{\mathcal{N}}$ and $\tilde{\mathcal{M}}$ in (7.9)-(7.10) with the new definitions in (E.10)- (E.11). In the next section, we demonstrate that the reduced-order model (E.12) obtained using the above formulation is guaranteed to be passive.

\section{E.3 Proof of passivity preservation}

As discussed in Chapter 7, in order to prove that the reduced model is passive, we just need to prove that the admittance matrix, $\tilde{\boldsymbol{Y}}(s)$, for the network represented by (E.12), satisfies the following condition.

$$
\boldsymbol{z}^{\star T}\left[\tilde{\boldsymbol{Y}}^{T}\left(s^{\star}\right)+\tilde{\boldsymbol{Y}}(s)\right] \boldsymbol{z} \geq 0 \quad \forall s \in\{s \mid \Re\{s\}>0\} \text { and } \forall \boldsymbol{z}
$$

To prove that this condition is satisfied, we can write the above condition using (E.12) as

$$
\boldsymbol{z}^{\star T}\left[\tilde{\boldsymbol{Y}}^{T}\left(s^{\star}\right)+\tilde{\boldsymbol{Y}}(s)\right] \boldsymbol{z}=\hat{\overline{\boldsymbol{F}}}^{T}\left(s^{*}\right) \hat{\overline{\mathbf{\Phi}}}(s) \hat{\overline{\boldsymbol{F}}}(s)
$$

where $\hat{\overline{\boldsymbol{F}}}(s)=\boldsymbol{F}(s) \hat{\overline{\boldsymbol{b}}}, \boldsymbol{F}(s)=(\hat{\overline{\mathcal{N}}}+s \hat{\overline{\mathcal{M}}})^{-1}$ and $\hat{\overline{\boldsymbol{\Phi}}}(s)=\hat{\overline{\mathcal{N}}}+s \hat{\overline{\mathcal{M}}}+\hat{\mathcal{\mathcal { N }}}^{T}+s^{*} \hat{\overline{\mathcal{M}}}^{T}$. From (E.13) it is evident that, to prove the passivity of the reduced model, it is required to prove that the matrix $\hat{\overline{\mathbf{\Phi}}}(s)$ is non-negative definite. For this purpose, using the definitions of $\hat{\overline{\mathcal{N}}}_{1}$ and $\hat{\overline{\mathcal{N}}}_{2}$ in Chapter 7 and substituting $s=\sigma+\jmath \omega$, where $\sigma>0$, matrix $\hat{\overline{\mathbf{\Phi}}}(s)$ can 
be rearranged as

$$
\hat{\bar{\Phi}}(s)=\hat{\overline{\mathcal{N}}}_{1}+\hat{\overline{\mathcal{N}}}_{1}^{T}+\hat{\overline{\mathcal{N}}}_{2}+\hat{\overline{\mathcal{N}}}_{2}^{T}+\sigma\left(\hat{\overline{\mathcal{M}}}+\hat{\overline{\mathcal{M}}}^{T}\right)+j \omega\left(\hat{\overline{\mathcal{M}}}-\hat{\overline{\mathcal{M}}}^{T}\right)
$$

Next, noting that $\hat{\overline{\mathcal{N}}}_{2}=-\hat{\overline{\mathcal{N}}}_{2}^{T}, \hat{\overline{\mathbf{\Phi}}}(s)$ can be rewritten as

$$
\hat{\overline{\mathbf{\Phi}}}(s)=\hat{\mathcal{\mathcal { N }}}_{1}+\hat{\mathcal{\mathcal { N }}}_{1}^{T}+\sigma\left(\hat{\overline{\mathcal{M}}}+\hat{\overline{\mathcal{M}}}^{T}\right)+\jmath \omega\left(\hat{\overline{\mathcal{M}}}-\hat{\overline{\mathcal{M}}}^{T}\right)
$$

Further, from its definition, $\hat{\overline{\mathcal{M}}}$ can be expressed as

$$
\begin{aligned}
\hat{\mathcal{M}} & =\int_{0}^{d} \mathcal{Q}(x)^{T} \tilde{\mathcal{M}} \mathcal{Q}(x) d x \\
& =\int_{0}^{d} \mathcal{Q}(x)^{T} \underbrace{\left[\begin{array}{cccc}
\mathbb{E}_{z} & \mathbf{0} & \mathbf{0} & \mathbf{0} \\
\mathbf{0} & \mathbb{E}_{y} & \mathbf{0} & \mathbf{0} \\
\mathbf{0} & \mathbf{0} & \boldsymbol{Q}_{z} & \mathbf{0} \\
\mathbf{0} & \mathbf{0} & \mathbf{0} & \boldsymbol{Q}_{z}
\end{array}\right]}_{\text {symmetric non-negative definite }} \mathcal{Q}(x) d x
\end{aligned}
$$

Where $\mathcal{Q}(x)$ is the orthonormal basis for the Hilbert subspace of the state variable vector $\tilde{\boldsymbol{X}}(x, s)$ in (E.9) and $d$ is the length of the interconnect. Noting that $\mathcal{Q}(x)$ is real [35], it is evident from (E.16) that $\hat{\overline{\mathcal{M}}}$ is symmetric non-negative definite, since it is given by the integration of congruence transformation of a non-negative definite matrix. As a result, $\hat{\bar{\Phi}}(s)$ can be rewritten as

$$
\hat{\bar{\Phi}}(s)=\hat{\overline{\mathcal{N}}}_{1}+\hat{\overline{\mathcal{N}}}_{1}^{T}+2 \sigma \hat{\overline{\mathcal{M}}}
$$

Hence, to prove that $\hat{\bar{\Phi}}(s)$ is non-negative definite, all that remains to be proved is that $\hat{\mathcal{\mathcal { N }}}_{1}+\hat{\overline{\mathcal{N}}}_{1}^{T}$ is non-negative definite. This can be done as follows: 


$$
\begin{aligned}
& \hat{\mathcal{\mathcal { N }}}_{1}+\hat{\overline{\mathcal{N}}}_{1}^{T}=\int_{0}^{d} \mathcal{Q}(x)^{T}\left(\tilde{\mathcal{N}}+\tilde{\mathcal{N}}^{T}\right) \mathcal{Q}(x) d x \\
& =\int_{0}^{d} \mathcal{Q}(x)^{T}\left[\begin{array}{cc}
\mathbb{D}_{z}+\mathbb{D}_{z}^{T} & \mathbf{0} \\
\mathbf{0} & \mathbb{D}_{y}+\mathbb{D}_{y}^{T} \\
\boldsymbol{C}_{z}^{T}-\boldsymbol{Q}_{z} \boldsymbol{B}_{z} & \mathbf{0} \\
\mathbf{0} & \boldsymbol{C}_{z}^{T}-\boldsymbol{Q}_{z} \boldsymbol{B}_{z}
\end{array}\right. \\
& \left.\begin{array}{cc}
\boldsymbol{C}_{z}-\boldsymbol{B}_{z}^{T} \boldsymbol{Q}_{z} & \mathbf{0} \\
\mathbf{0} & \boldsymbol{C}_{y}-\boldsymbol{B}_{y}^{T} \boldsymbol{Q}_{y} \\
-\boldsymbol{Q}_{z} \boldsymbol{A}_{z}-\boldsymbol{A}_{z}^{T} \boldsymbol{Q}_{z} & \mathbf{0} \\
\mathbf{0} & -\boldsymbol{Q}_{z} \boldsymbol{A}_{z}-\boldsymbol{A}_{z}^{T} \boldsymbol{Q}_{z}
\end{array}\right] \mathcal{Q}(x) d x \\
& =\int_{0}^{d} \mathcal{Q}(x)^{T} \boldsymbol{K}^{T}\left[\begin{array}{cc}
-\boldsymbol{Q}_{z} \boldsymbol{A}_{z}-\boldsymbol{A}_{z}^{T} \boldsymbol{Q}_{z} & \boldsymbol{C}_{z}^{T}-\boldsymbol{Q}_{z} \boldsymbol{B}_{z} \\
\boldsymbol{C}_{z}-\boldsymbol{B}_{z}^{T} \boldsymbol{Q}_{z} & \mathbb{D}_{z}+\mathbb{D}_{z}^{T} \\
\mathbf{0} & \mathbf{0} \\
\mathbf{0} & \mathbf{0}
\end{array}\right. \\
& \left.\begin{array}{cc}
\mathbf{0} & \mathbf{0} \\
\mathbf{0} & \mathbf{0} \\
-\boldsymbol{Q}_{y} \boldsymbol{A}_{y}-\boldsymbol{A}_{y}^{T} \boldsymbol{Q}_{y} & \boldsymbol{C}_{y}^{T}-\boldsymbol{Q}_{y} \boldsymbol{B}_{y} \\
\boldsymbol{C}_{y}-\boldsymbol{B}_{y}^{T} \boldsymbol{Q}_{y} & \mathbb{D}_{y}+\mathbb{D}_{y}^{T}
\end{array}\right] \boldsymbol{K \mathcal { Q }}(x) d x
\end{aligned}
$$

where, $\boldsymbol{K}=\left[\begin{array}{cccc}\mathbf{0} & \boldsymbol{I}_{N_{z}} & \mathbf{0} & \mathbf{0} \\ \mathbf{0} & \mathbf{0} & \mathbf{0} & \boldsymbol{I}_{N_{z}} \\ \boldsymbol{I}_{N_{y}} & \mathbf{0} & \mathbf{0} & \mathbf{0} \\ \mathbf{0} & \mathbf{0} & \boldsymbol{I}_{N_{y}} & 0\end{array}\right]$ and $\boldsymbol{I}_{N_{z}}$ and $\boldsymbol{I}_{N_{y}}$ are identity matrices with a dimension of $N_{z}$ and $N_{y}$, respectively. Since the systems (E.3) and (E.4) are positive-real, and $\boldsymbol{Q}_{z}, \boldsymbol{Q}_{y}$ represent the non-negative definite solutions of the LMIs in (E.5)-(E.6), respectively; and $\mathcal{Q}(x)$ is real, it can be inferred that $\hat{\mathcal{N}}_{1}+\hat{\overline{\mathcal{N}}}_{1}^{T}$ is non-negative definite. Hence the reduced system (E.12) is passive. 


\section{E.4 Complexity analysis}

In this section, the complexity analysis of constructing the reduced-order model based on the proposed formulation of TEs is discussed. In order to apply the ICT algorithm on the general TEs, such as in (E.9), we need L/U decomposition once and forward/backward substitution $q$ times for a $q$-order reduction [35]. Based on the general form of TEs proposed in this appendix, the size of the associated matrix for $\mathrm{L} / \mathrm{U}$ decomposition and forward/backward substitution is $N=H\left(2 m+N_{z}+N_{y}\right)$. Here, $H$ is the highest order of Chebyshev polynomials used to approximate the moments ( $x$-dependent). $m$ is the number of signal conductors of the TL. $N_{z}$ and $N_{y}$ represent the dimensions of the state-space representations associated with the rational functions used to approximate the frequency-dependent PUL impedances and admittances, respectively. This is compared to $N=H\left(2 m+n_{Z}+n_{Y}\right)$ when the form of TEs in Chapter 7 is used, where $n_{Z}$ and $n_{Y}$ are the sizes of MNA matrices of lumped networks of PUL impedances and admittances, which are synthesized from PR rational functions. Usually, $N_{z}, N_{y}$ are either smaller than or equal to $n_{Z}, n_{Y}$. This makes the reduction process more efficient based on the general formulation proposed in this appendix (while guaranteeing the passivity). It should be noted that the computational cost of solving for $\boldsymbol{Q}_{z}$ and $\boldsymbol{Q}_{\boldsymbol{y}}$ is very small (in the range of fractions of a second), since the size of the system (E.3)-(E.4) is usually small for the state-space system obtained from the approximation of FD-PUL parameters.

\section{E.5 Numerical examples}

In order to show the effectiveness of the proposed method, a two-conductor TL with frequency-dependent PUL parameters is considered. The PUL impedance $\mathcal{Z}(s)$ and admittance $\mathcal{Y}(s)$ matrices were approximated with a 5 -th order PR rational function $\tilde{\mathcal{Z}}(s)$ 
and $\tilde{\mathcal{Y}}(s)$, as described in (E.1) and (E.2), respectively. Next, using the proposed algorithm as outlined in Section E.2, the general TEs of order $12\left(=2 m+N_{z}+N_{y}\right)$ were obtained. Further, using ICT on the resulting TEs, a reduced model of order 60 was constructed as per (E.12). Figure E.1 and Figure E.2 show the comparison of the corresponding $\left|Y_{11}\right|$ and $\left|Y_{12}\right|$, respectively, with that obtained by solving the TEs directly in the frequencydomain (exact). As seen they match well with the exact response. The corresponding plot of the eigenvalue of the real part of the admittance matrix is given in Figure E.3. As seen the eigenvalue of the proposed reduced model (shown as curve 1) remains non-negative through out the frequency spectrum, indicating that the model is passive. Passivity is also confirmed by using the alternative method of Hamiltonian matrix based formulation described in [59]. On the other hand, when the state-space equations (E.3)-(E.4) were directly included in (2.1) without using the proposed algorithm (i.e. mapping matrices $\boldsymbol{Q}_{z}$ and $Q_{y}$ are not used for pre-multiplication as in (E.7)-(E.8)) and the reduced order model (E.12) was subsequently obtained, it was found that the eigenvalue of the real part of the admittance matrix of the reduced model was negative (i.e. the model was non-passive), as demonstrated by curve 2 in Figure E.3. The proposed method required a total macromodeling time of 7.8 seconds (including 0.09 seconds for the computation of $\boldsymbol{Q}_{z}$ and 0.02 seconds for $\boldsymbol{Q}_{y}$ ) in MATLAB ${ }^{\circledR}$ on a WindowXp platform running on a PC Pentium IV $(1.5 \mathrm{GHz})$ processor. 


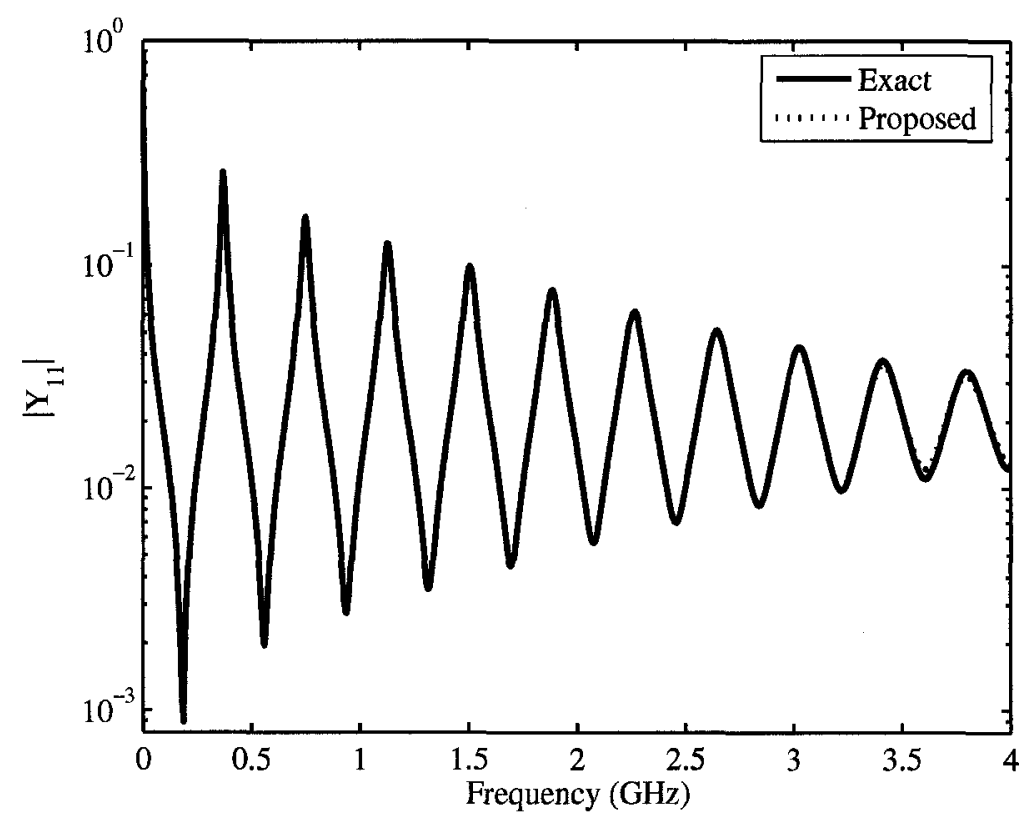

Figure E.1: Accuracy comparison of the proposed method, $\left|\boldsymbol{Y}_{11}\right|$.

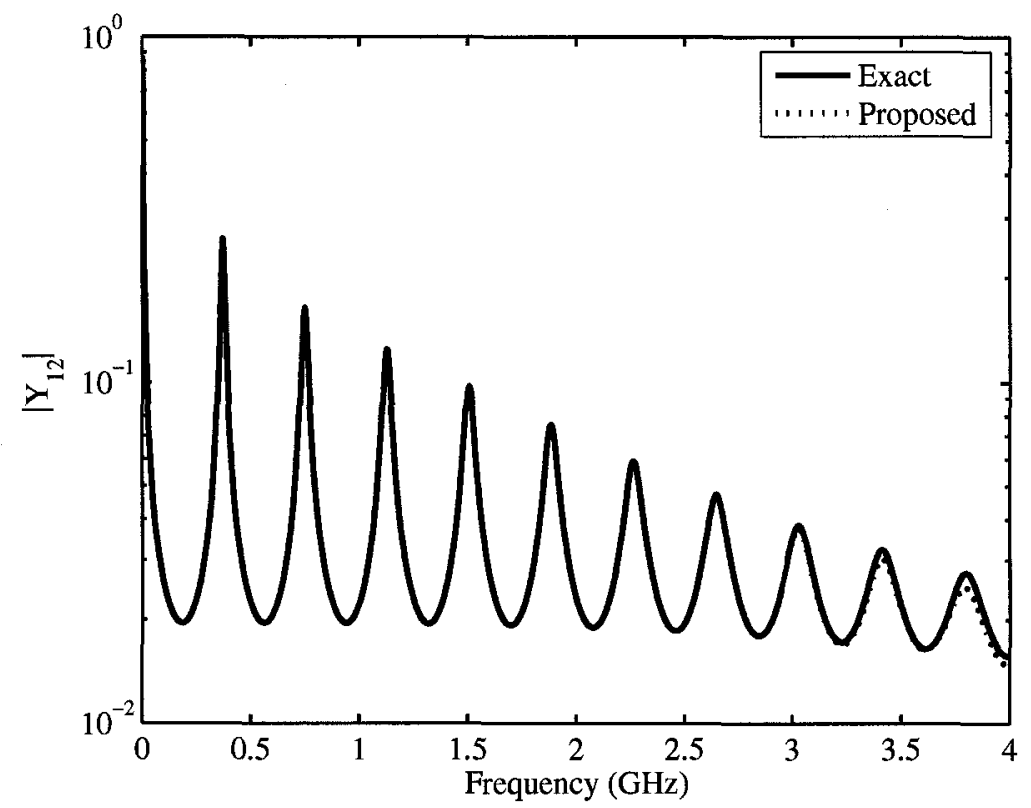

Figure E.2: Accuracy comparison of the proposed method, $\left|\boldsymbol{Y}_{12}\right|$. 


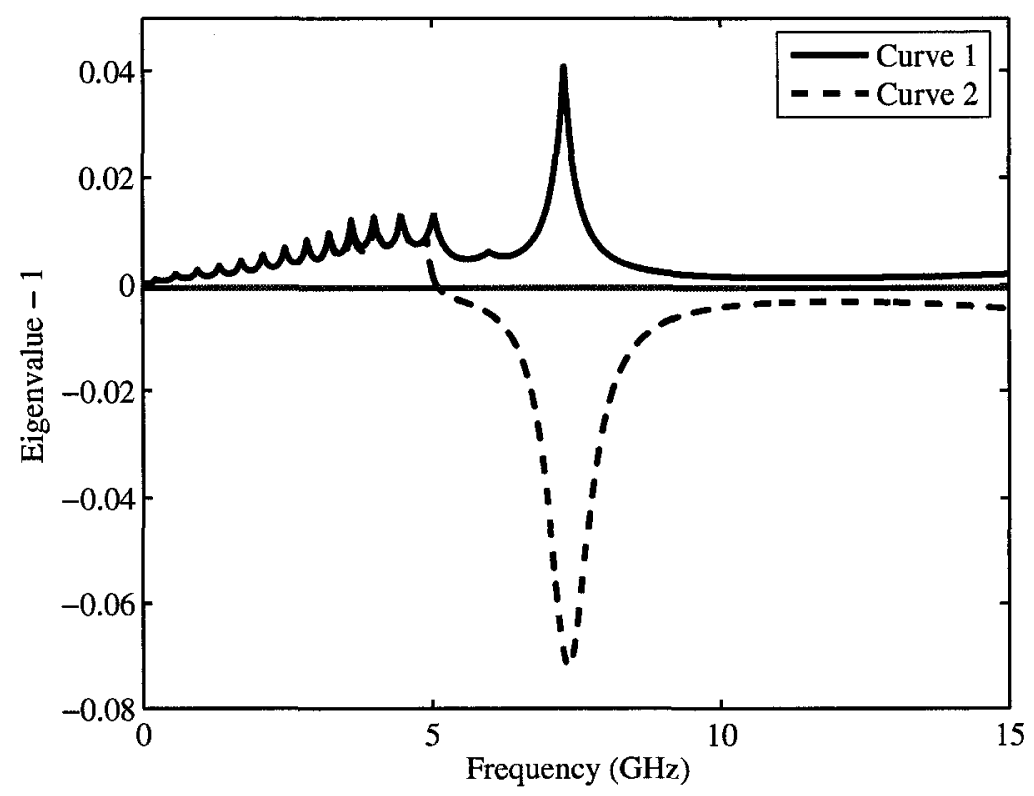

Figure E.3: Eigenvalue plot of the real part of admittance matrix. 


\section{Appendix F}

\section{Proof of Proposition 8.3.4}

The proof of Proposition 8.3.4 is stimulated from the MoC model in the Laplace-domain for a lossless $(m+1)$-conductor TL as described in (8.2), which is rewritten in the following,

$$
\left\{\begin{array}{l}
\boldsymbol{V}_{n 1}(s)=\mathcal{Z}_{o} \boldsymbol{I}_{n 1}(s)+e^{-s \sqrt{\boldsymbol{L C}} d}\left(\mathcal{Z}_{o} \boldsymbol{I}_{n 2}(s)+\boldsymbol{V}_{n 2}(s)\right) \\
\boldsymbol{V}_{n 2}(s)=\mathcal{Z}_{o} \boldsymbol{I}_{n 2}(s)+e^{-s \sqrt{\boldsymbol{L C}}}\left(\boldsymbol{\mathcal { Z }}_{o} \boldsymbol{I}_{n 1}(s)+\boldsymbol{V}_{n 1}(s)\right)
\end{array}\right.
$$

By introducing two new variables, $\boldsymbol{X}_{1}(s)$ and $\boldsymbol{X}_{2}(s)$ defined by,

$$
\left\{\begin{array}{l}
\boldsymbol{V}_{n 1}(s)=\mathcal{Z}_{o} \boldsymbol{X}_{1}(s) \\
\boldsymbol{V}_{n 2}(s)=\mathcal{Z}_{o} \boldsymbol{X}_{2}(s)
\end{array}\right.
$$

(F.1) can be rewritten as,

$$
\left\{\begin{array}{l}
-\frac{1}{2} \mathcal{Z}_{o} \boldsymbol{I}_{n 1}+\frac{1}{2} \mathcal{Z}_{o} \boldsymbol{X}_{1}-\frac{1}{2} e^{-s \sqrt{L \boldsymbol{C}} d} \mathcal{Z}_{o} \boldsymbol{I}_{n 2}-\frac{1}{2} e^{-s \sqrt{\boldsymbol{L C}} d} \mathcal{Z}_{o} \boldsymbol{X}_{2}=0 \\
-\frac{1}{2} \mathcal{Z}_{o} \boldsymbol{I}_{n 2}+\frac{1}{2} \mathcal{Z}_{o} \boldsymbol{X}_{2}-\frac{1}{2} e^{-s \sqrt{\boldsymbol{L C}} d} \mathcal{Z}_{o} \boldsymbol{I}_{n 1}-\frac{1}{2} e^{-s \sqrt{\boldsymbol{L C}} d} \mathcal{Z}_{o} \boldsymbol{X}_{1}=0
\end{array}\right.
$$

and

$$
\begin{cases}-\frac{1}{2} \mathcal{Z}_{o} \boldsymbol{I}_{n 1}-\frac{1}{2} \mathcal{Z}_{o} \boldsymbol{X}_{1}+\frac{1}{2} e^{-s \sqrt{L \boldsymbol{C}} d} \mathcal{Z}_{o} \boldsymbol{I}_{n 2}+\frac{1}{2} e^{-s \sqrt{\boldsymbol{L C}} d} \mathcal{Z}_{o} \boldsymbol{X}_{2}+\mathcal{Z}_{o} \boldsymbol{I}_{n 1}= & 0 \\ -\frac{1}{2} \mathcal{Z}_{o} \boldsymbol{I}_{n 2}-\frac{1}{2} \mathcal{Z}_{o} \boldsymbol{X}_{2}+\frac{1}{2} e^{-s \sqrt{L \boldsymbol{C}} d} \mathcal{Z}_{o} \boldsymbol{I}_{n 1}+\frac{1}{2} e^{-s \sqrt{\boldsymbol{L C}} d} \mathcal{Z}_{o} \boldsymbol{X}_{1}+\mathcal{Z}_{o} \boldsymbol{I}_{n 2}= & 0\end{cases}
$$

To simplify the presentation, the explicit function representation of " $s$ " for $\boldsymbol{I}_{n 1}(s), \boldsymbol{I}_{n 2}(s)$, $\boldsymbol{V}_{n 1}(s), \boldsymbol{V}_{n 2}(s), \boldsymbol{X}_{1}(s)$ and $\boldsymbol{X}_{2}(s)$, is neglected. (F.2)-(F.4) can then be combined and 
rewritten in the following matrix form,

$$
\begin{aligned}
\mathbf{0} & =\boldsymbol{A} \boldsymbol{\rho}(s)+\boldsymbol{A}_{s}(s) \boldsymbol{\rho}(s)+\boldsymbol{B} \boldsymbol{I}(s) \\
\boldsymbol{V}(s) & =\boldsymbol{B}^{T} \boldsymbol{\rho}(s)
\end{aligned}
$$

where $\boldsymbol{A}$ and $\boldsymbol{B}$ are defined in the proposition statement; $\boldsymbol{\rho}(s)=\left[\begin{array}{llll}\boldsymbol{I}_{n 1}^{T} & \boldsymbol{I}_{n 2}^{T} & \boldsymbol{X}_{1}^{T} & \boldsymbol{X}_{2}^{T}\end{array}\right]^{T}$;

$$
\begin{aligned}
\boldsymbol{I}_{t l}(s)=\left[\begin{array}{ll}
\boldsymbol{I}_{n 1}^{T} & \boldsymbol{I}_{n 2}^{T}
\end{array}\right]^{T} ; \boldsymbol{V}_{t l}(s)=\left[\begin{array}{lll}
\boldsymbol{V}_{n 1}^{T} & \boldsymbol{V}_{n 2}^{T}
\end{array}\right]^{T} \text { and } \\
\boldsymbol{A}_{s}(s)=\left[\begin{array}{cccc}
\mathbf{0} & -\frac{1}{2} e^{-s \sqrt{\boldsymbol{L C}} d} \mathcal{Z}_{o} & \mathbf{0} & -\frac{1}{2} e^{-s \sqrt{\boldsymbol{L C}} d} \mathcal{Z}_{o} \\
-\frac{1}{2} e^{-s \sqrt{\boldsymbol{L C}} d} \mathcal{Z}_{o} & \mathbf{0} & -\frac{1}{2} e^{-s \sqrt{\boldsymbol{L C}} d} \mathcal{Z}_{o} & \mathbf{0} \\
\mathbf{0} & \frac{1}{2} e^{-s \sqrt{\boldsymbol{L} \boldsymbol{C}} d} \mathcal{Z}_{o} & \mathbf{0} & \frac{1}{2} e^{-s \sqrt{\boldsymbol{L C}} d} \mathcal{Z}_{o} \\
\frac{1}{2} e^{-s \sqrt{\boldsymbol{L C}} d} \mathcal{Z}_{o} & \mathbf{0} & \frac{1}{2} e^{-s \sqrt{\boldsymbol{L C}} d} \mathcal{Z}_{o} & \mathbf{0}
\end{array}\right]
\end{aligned}
$$

Given the fact that $\sqrt{L C} d=\mathfrak{M} \tau \mathfrak{M}^{-1}$, (F.7) can be simplified, after some mathematical manipulations, to be,

$$
\boldsymbol{A}_{s}(s)=\frac{1}{2} \mathfrak{M}\left[\begin{array}{cccc}
\mathbf{0} & -e^{-s \tau} & \mathbf{0} & -e^{-s \tau} \\
-e^{-s \boldsymbol{\tau}} & \mathbf{0} & -e^{-s \boldsymbol{\tau}} & \mathbf{0} \\
\mathbf{0} & e^{-s \tau} & \mathbf{0} & e^{-s \tau} \\
e^{-s \tau} & \mathbf{0} & e^{-s \boldsymbol{\tau}} & \mathbf{0}
\end{array}\right] \mathfrak{M}^{-1} \mathcal{Z}_{o}=\sum_{k=1}^{m} \boldsymbol{A}_{k} e^{-s \tau_{k}}
$$

Here $\boldsymbol{A}_{k}$ is same defined as in the proposition statement. By substituting (F.8) into (F.5)(F.6), and writing in the time-domain form, we shall obtain (8.3). Therefore, the proof is complete. 


\section{Appendix G}

\section{Proof of Lemma 8.3.9}

Proof Proposition 8.3.4 demonstrates that (8.3) is the state-space representation for a lossless transmission line, with its transfer function being $\hat{\mathbf{\Pi}}(s)$ in (8.4). Given that a lossless transmission line is passive [10], $\hat{\Pi}(s)$ satisfies

$$
\boldsymbol{z}^{\star T}\left[\hat{\boldsymbol{\Pi}}(s)+\hat{\boldsymbol{\Pi}}^{T}\left(s^{\star}\right)\right] \boldsymbol{z} \geq 0 \forall s \in\{s \mid \Re\{s\}>0\} \text { and } \forall \boldsymbol{z}
$$

Substituting $\hat{\Pi}(s)$ into (G.1) yields,

$$
\begin{aligned}
& \boldsymbol{z}^{\star T}\left[\hat{\boldsymbol{\Pi}}(s)+\hat{\boldsymbol{\Pi}}^{T}\left(s^{\star}\right)\right] \boldsymbol{z} \\
= & \boldsymbol{z}^{\star T}\left[\boldsymbol{B}^{T}\left(-\boldsymbol{A}-\sum_{k=1}^{m} \boldsymbol{A}_{k} e^{-s \tau_{k}}\right)^{-1} \boldsymbol{B}+\boldsymbol{B}^{T}\left(-\boldsymbol{A}^{T}-\sum_{k=1}^{m} \boldsymbol{A}_{k}^{T} e^{-s^{\star} \tau_{k}}\right)^{-1} \boldsymbol{B}\right] \boldsymbol{z} \\
= & \hat{\boldsymbol{z}}^{\star T}\left[-\boldsymbol{A}-\sum_{k=1}^{m} \boldsymbol{A}_{k} e^{-s \tau_{k}}-\boldsymbol{A}^{T}-\sum_{k=1}^{m} \boldsymbol{A}_{k}^{T} e^{-s^{\star} \tau_{k}}\right] \hat{\boldsymbol{z}} \geq 0
\end{aligned}
$$

where $\hat{\boldsymbol{z}}=\left(-\boldsymbol{A}-\sum_{k=1}^{m} \boldsymbol{A}_{k} e^{-s \tau_{k}}\right)^{-1} \boldsymbol{B} z$. (G.2) shows that

$$
\left(-\boldsymbol{A}-\sum_{k=1}^{m} \boldsymbol{A}_{k} e^{-s \tau_{k}}-\boldsymbol{A}^{T}-\sum_{k=1}^{m} \boldsymbol{A}_{k}^{T} e^{-s^{\star} \tau_{k}}\right)
$$

is non-negative definite and the proof is done. 


\section{References}

[1] R. Achar and M. Nakhla, "Simulation of high-speed interconnects," Proceedings IEEE, vol. 89, pp. 693-728, May 2001.

[2] C. Paul, Analysis of Multiconductor Transmission Lines. New York: Wiley, 1994.

[3] T. Dhaene and D. Zutter, "Selection of lumped element models for coupled lossy transmission lines," IEEE Trans. Computer-Aided Design, vol. 11, pp. 805-815, July 1992.

[4] A. Dounavis, Passive Time Domain Macromodels for High-Speed Interconnect Networks. PhD thesis, Carleton University, 2003.

[5] F. H. Branin, Jr., "Transient analysis of lossless transmission lines," Proceedings IEEE, pp. 2012-2013, 1967.

[6] D. Kuznetsov and J. E. Schutt-Aine, "Optimal transient simulation of transmission lines,” IEEE Trans. Circuits Syst., vol. 43, pp. 110-121, Feb. 1996.

[7] A. Gruodis and C. Chang, "Coupled lossy transmission line characterization and simulation," IBM Journal of Research and Development, vol. 25, no. 1, pp. 25-41, 1981. 
[8] S. Lin and E. Kuh, "Transient simulation of lossy interconnects based on the recursive convolution formulation," IEEE Trans. Circuits Syst. I, vol. 39, pp. 879-892, Nov. 1992.

[9] S. Grivet-Talocia, H.-M. Huang, A. E. Ruehli, F. Canavero, and I. Elfadel, "Transient analysis of lossy transmission lines: An efficient approach based on the method of characteristics," IEEE Trans. Adv. Packag., vol. 27, no. 1, pp. 45-56, 2004.

[10] N. Nakhla, A. Dounavis, R. Achar, and M. Nakhla, "DEPACT: Delay extractionbased passive compact transmission-line macromodeling algorithm," IEEE Trans. Adv. Packag., vol. 28, pp. 13-23, February 2005.

[11] F. Fer, "Resolution de l'equation matricielle dU/dt $=\mathrm{pU}$ par produit infini d'exponentielles matricielles," Acad. Roy. Belg. Cl. Sci., vol. 44, no. 5, pp. 815-829, 1958.

[12] L. T. Pillage and R. A. Rohrer, "Asymptotic waveform evaluation for timing analysis," IEEE Trans. Computer-Aided Design, vol. 9, pp. 352-366, Apr. 1990.

[13] P. Feldmann and R. W. Freund, "Efficient linear circuit analysis by Padé via Lanczos process," IEEE Trans. Computer-Aided Design, vol. 14, pp. 639-649, May 1995.

[14] A. Odabasioglu, M. Celik, and L. T. Pileggi, "PRIMA: passive reduced-order interconnect macromodeling algorithm," IEEE Trans. Computer-Aided Design, vol. 17, pp. 645-654, Aug. 1998.

[15] R. W. Freund and P. Feldmann, "The SyMPVL algorithm and its applications to interconnect simulation," in Proc. Intl. Conf. on Simulation of Semiconductor Processes and Devices, pp. 113-116, Sept. 1997. 
[16] K. J. Kerns and A. T. Yang, "Preservation of passivity during RLC network reduction via split congruence transformations," IEEE Trans. Computer-Aided Design, vol. 17, pp. 582-591, July 1998.

[17] I. M. Elfadel and D. D. Ling, "A block rational Arnoldi algorithm for multipoint passive model-order reduction of multiport RLC networks," in Proc. IEEE/ACM Intl. Conf. Computer-Aided Design, pp. 66-71, 1997.

[18] J. Phillips, "Variational interconnect analysis via PMTBR," in Proc. IEEE/ACM Intl. Conf. Computer-Aided Design, pp. 872-879, Nov. 2004.

[19] J. Phillips, L. Daniel, and L. Silveira, "Guaranteed passive balancing transformations for model order reduction," IEEE Trans. Computer-Aided Design of Integrated Circuit and Systems, vol. 22, pp. 1027-1041, Aug. 2003.

[20] Q. Yu, J. M. L. Wang, and E. S. Kuh, "Passive multipoint moment matching model order reduction algorithm on multiport distributed interconnect networks," IEEE Trans. Circuits Syst. I, vol. 46, pp. 140-160, Jan. 1999.

[21] E. Gad and M. Nakhla, "Efficient analysis of non-uniform transmission lines via integrated congruent transform," IEEE Trans. Very Large Scale Integration (VLSI) Systems, vol. 12, pp. 1307-1320, Dec 2004.

[22] E. Gad and M. Nakhla, "Simulation and sensitivity analysis of nonuniform transmission lines using integrated congruence transform," IEEE Trans. Adv. Packag., vol. 28, pp. 32-44, Feb 2005.

[23] M. Celik and A. Cangellaris, "Efficient transient simulation of lossy packaging interconnects using moment-matching techniques," IEEE Trans. Components, Packaging, and Manufacturing Tech.-Part B, vol. 19, pp. 64-73, Feb. 1996. 
[24] P. Silvester, "Model network theory of skin effect in flat conductors," in Proceedings IEEE, vol. 54, pp. 1147-1151, 1966.

[25] S. Mei and Y. Ismail, "Modeling skin and proximity effects with reduced realizable RL circuits," IEEE Trans. Very Large Scale Integration (VLSI) Systems, vol. 12, pp. 437-447, April 2004.

[26] T. Wuyts and D. Zutter, "Circuit model for plane-wave incidence on multiconductor transmission lines," IEEE Trans. Electromag. Compat., vol. 36, pp. 206-212, Aug. 1994.

[27] T. Vu Dinh, B. Cabon, and J. Chilo, "Time domain analysis of skin effect on lossy interconnects," Electronics Letters, vol. 26, pp. 2057-2058, Sept. 1990.

[28] D. Saraswat, R. Achar, and M. Nakhla, "Passive reduction algorithm for RLC interconnect circuits with embedded state-space systems (PRESS)," IEEE Trans. Microwave Theory Tech., vol. 52, pp. 2215-2226, September 2004.

[29] P. Feldmann, "Model order reduction techniques for linear systems with large numbers of terminals," in Proc. Design Automation and Test in Europe (DATE), pp. 944947, 2004.

[30] E. Gad, C. Chen, M. Nakhla, and R. Achar, "Passivity verification in delay-based macromodels of electrical interconnects," IEEE Trans. Circuits Syst. I, vol. 52, pp. 2173-2187, Oct. 2005.

[31] C. Chen, E. Gad, M. Nakhla, and R. Achar, "Passivity verification in delaybased macromodels of multi-conductor electrical interconnects," IEEE Trans. Adv. Packag., vol. 30, pp. 246-256, May 2007. 
[32] E. Gad, C. Chen, M. Nakhla, and R. Achar, "A passivity checking algorithm for delay-based macromodels of lossy transmission lines," in Proc. IEEE workshop on Signal Propagation on Interconnects SPI, May 2005.

[33] C. Chen, D. Saraswat, R. Achar, E. Gad, M. Nakhla, and M. Yagoub, "Passivity compensation algorithm for method of characteristics-based multiconductor transmission line interconnect macromodels," submitted to IEEE Trans. on VLSI, 2007.

[34] C. Chen, D. Saraswat, E. Gad, R. Achar, M. Nakhla, and M. Yagoub, "Passivity enforcement for method of characteristics-based macromodels," in Int. Symposium on Signals, Systems and Electronics (ISSSE), (Montreal, Canada), July 2007.

[35] C. Chen, E. Gad, M. Nakhla, and R. Achar, "Analysis of frequency-dependent interconnects using integrated congruence transform," IEEE Trans. Computer-Aided Design, vol. 26, pp. 1139-1149, June 2007.

[36] N. Nakhla, A. Ruehlit, M. Nakhla, R. Achar, and C. Chen, "Waveform relaxation techniques for simulation of coupled interconnects with frequency-dependent parameters," IEEE Trans. Adv. Packag., vol. 30, pp. 257-269, May 2007.

[37] C. Chen, D. Saraswat, R. Achar, E. Gad, M. Nakhla, and M. Yagoub, "A robust algorithm for passive reduced-order macromodeling of MTLs with FD-PUL parameters using integrated congruence transform," Accepted for publication in IEEE Trans. on Computer-Aided Design, 2007.

[38] C. Chen, E. Gad, M. Nakhla, and Y. Guo, "Analysis of frequency dependent transmission lines using integrated congruence transform," in in Proceedings of the Annual Review of Progress in Applied Computational Electromagnetics, April 2004. 
[39] C. Chen, E. Gad, M. Nakhla, R. Achar, and W. Tseng, "Model-order reduction of frequency-dependent interconnects based on integrated congruence transform," in IEEE Int. Midwest Symposium on Circuits and Systems (MWSCAS), (Montreal, Canada), Aug. 2007.

[40] W. Tseng, C. Chen, E. Gad, M. Nakhla, and R. Achar, "Passive order reduction for RLC circuits with delay elements," IEEE Trans. Adv. Packag., 2007. Accepted for publish.

[41] C. Chen, E. Gad, W. Tseng, M. Nakhla, and R. Achar, "A passive model-order reduction algorithm for RLC networks with embedded delay elements," in Proc. IEEE workshop on Signal Propagation on Interconnects SPI, May 2006.

[42] C. Chen, E. Gad, M. Nakhla, R. Achar, and W. Tseng, "Passive model-order reduction for rlc systems with delay elements," in North American Radio Science Meeting (URSI), (Ottawa, Canada), July 2007.

[43] A. Ruehli and A. Cangellaris, "Progress in the methodologies for the electrical modeling of interconnects and electronic packages," in Proceedings of IEEE, vol. 89, pp. $740-771,2001$.

[44] D. Saraswat, "Passive macromodeling of linear subnetworks characterized by measured simulated data," Master's thesis, Carleton University, 2003.

[45] E. S. Kuh and R. A. Rohrer, Theory of Linear Active Networks. San Francisco: Holden-Day, 1967.

[46] B. Anderson and S. Vongpanitlerd, Network Analysis and Synthesis. A Modern System Theory Approach. Prentice-Hall, Inc., 1973. 
[47] L. Weinberg, Network Analysis and Synthesis. New York: McGraw-Hill,, 1962.

[48] A. E. Guillemin, Synthesis of Passive Networks. NY:John Wiley and Son Inc., 1957.

[49] J. Marsden, B. M., H. M., and R. C., Basic Complex Analysis. San Francisco: W. H. Freeman, 1973.

[50] A. Jeffrey, Complex analysis and applications. Boca Raton, FL: CRC Press, 1992.

[51] A. Dounavis, R. Achar, and M. Nakhla, "Efficient passive circuit models for distributed networks with frequency-dependent parameters," IEEE Trans. Adv. Packag., vol. 23, pp. 382-392, Aug. 2000.

[52] M. Celik, A. C. Cangellaris, and A. Yaghmour, "An all purpose transmission line model for interconnect simulation in SPICE," IEEE Trans. Microwave Theory Tech., vol. 45, pp. 1857-1867, Oct. 1997.

[53] E. Gad, A. Dounavis, M. Nakhla, and R. Achar, "Passive model order reduction of multiport distributed interconnects," in Proc. IEEE/ACM Design Automation Conf., pp. 526-531, Jul. 2000.

[54] A. Dounavis, I. Elfadel, R. Achar, M. Nakhla, A. Ruehli, and H.-M. Huang, "Lossy transmission line passive macromodeling algorithm - three case studies," in IEEE Topical Meeting on Electrical Performance of Electronic Packaging, pp. 355-358, Oct 2002.

[55] Y.-S. Guo, "Transient simulation of high-speed interconnects based on the semidiscretization of Telegrapher's equations," IEEE Trans. Computer-Aided Design of Integrated Circuit and Systems, vol. 21, pp. 799-809, July 2002. 
[56] M. Celik and A. C. Cangellaris, "Simulation of multiconductor transmission lines using Krylov subspace order-reduction techniques," IEEE Trans. Computer-Aided Design, vol. 16, pp. 485-496, May 1997.

[57] A. Dounavis, R. Achar, and M. S. Nakhla, "On passive time-domain macromodels of distributed transmission line networks," in IEEE MTT-S Intl. Microwave Symp. Dig., pp. 975-978, June 2002.

[58] N. Nakhla, A. Dounavis, M. Nakhla, and R. Achar, "Delay-extraction based sensitivity analysis of multiconductor transmission lines with nonlinear terminations," in IEEE MTT-S Intl. Microwave Symp. Dig., pp. 1427-1430, June 2005.

[59] D. Saraswat, R. Achar, and M. Nakhla, "Global passivity enforcement algorithm for macromodels of interconnect subnetworks characterized by tabulated data," IEEE Trans. Very Large Scale Integration (VLSI) Systems, vol. 45, pp. 819-832, Jul. 2005.

[60] C. Coehlo, J. Phillips, and L. Silveira, "A convex programming approach for generating guaranteed passive approximations to tabulated frequency-data," IEEE Trans. Computer-Aided Design of Integrated Circuit and Systems, vol. 23, pp. 293-301, Feb. 2004.

[61] B. Gustavsen and A. Semlyen, "Rational approximation of frequency responses by vector fitting," IEEE Trans. on Power Delivery, vol. 14, pp. 1052-1061, 1999.

[62] W. Beyene and J. Schutt-Aine, "Efficient transient simulation of high-speed interconnects characterized by sampled data," IEEE Trans. Components, Packaging, and Manufacturing Tech.-Part B, vol. 21, pp. 105-114, Feb. 1998. 
[63] M. Elzinga, K. Virga, L. Zhao, and J. Prince, "Pole-residue formulation for transient simulation of high-frequency interconnects using householder LS curve-fitting techniques," IEEE Trans. Adv. Packag., vol. 25, pp. 142-147, May 2000.

[64] S. Grivet-Talocia, "Passivity enforcement via perturbation of Hamiltonian matrices," IEEE Trans. Circuits Syst. I, vol. 51, no. 9, pp. 1755-1769, 2004.

[65] S. Boyd, L. El Ghaoui, E. Feron, and V. Balakrishnan, Linear Matrix Inequalities in System and Control Theory, vol. 15. Philadelphia, PA: SIAM, 1994.

[66] D. Saraswat, R. Achar, and M. Nakhla, "Fast passivity verification and enforcement via reciprocal systems for interconnects with large order macromodels," IEEE Trans. Very Large Scale Integration (VLSI) Systems, vol. 15, pp. 48-58, Jan. 2007.

[67] G. A. Baker Jr., Essential of Padé approximants. New York: Academic Press, 1975.

[68] E. Chiprout and M. Nakhla, Asymptotic Waveform Evaluation and Moment Matching for Interconnect Analysis. Boston: Kluwer, 1993.

[69] Q. Yu and E. Kuh, "Exact moment-matching model of transmission lines and application to interconnect delay estimation," IEEE Trans. Very Large Scale Integration (VLSI) Systems, pp. 311-322, Jun 1995.

[70] J. E. Bracken, V. Raghavan, and R. A. Rohrer, "Interconnect simulation with asymptotic waveform evaluation," IEEE Trans. Circuits Syst., vol. 39, pp. 869-878, Nov. 1990.

[71] E. Chiprout and M. Nakhla, "Analysis of interconnect networks using complex frequency hopping (CFH)," IEEE Trans. Computer-Aided Design, vol. 14, pp. 186-200, Feb. 1995. 
[72] R. Achar, M. Nakhla, and Q. Zhang, "Full-wave analysis of high-speed interconnects using complex frequency hopping," IEEE Trans. Computer-Aided Design, vol. 17, no. 10 , pp. 997-1015, 1998.

[73] R. Achar, M. Nakhla, and E. Chiprout, "Block CFH: A model-reduction technique for distributed interconnect networks," IEEE Trans. Microwave Theory Tech., vol. 42, pp. 2562-2571, Dec. 1994.

[74] T. V. Nguyen and J. Li, "Multipoint Padé approximation using a rational block lanczos algorithm," in Proc. IEEE/ACM Intl. Conf. Computer-Aided Design, pp. 72-75, 1997.

[75] E. Celledoni and I. Moret, "A Krylov projection method for systems of ODEs," Applied Numerical Mathematics, vol. 24, pp. 365-378, 1997.

[76] M. Kamon, F. Wang, and J. White, "Generating nearly optimally compact models from Krylov subspace based reduced-order models," IEEE Trans. Circuits Syst. II, vol. 47, pp. 239-248, Apr. 2000.

[77] R. Khazaka and M. Nakhla, "Addressing redundant poles in Krylov based model reduction," in Proc. IEEE workshop on Signal Propagation on Interconnects SPI, pp. 1-4, May 2001.

[78] M. Celik, L. Pileggi, and A. Odabasioglu, IC Interconnect Analysis. Kluwer Academic Publishers, 2002.

[79] B. Moore, "Principal component analysis in linear systems: controllability, observability and model reduction," IEEE Trans. Automatic Control, vol. 26, pp. 17-32, Feb 1981. 
[80] X. Chen and J. Wen, "Positive realness preserving model reduction with $\mathrm{H}_{\infty}$ norm error bound," IEEE Trans. Circuits Syst. I, vol. 42, pp. 23-29, Jan 1995.

[81] P. K. Gunupudi, M. Nakhla, and A. Ramachandra, "Simulation of high-speed distributed interconnects using Krylov-space techniques," IEEE Trans. ComputerAided Design, vol. 19, pp. 799-807, July 2000.

[82] P. Gunupudi, M. Nakhla, and R. Achar, "Simulation of high-speed distributed interconnects using krylov-space techniques," IEEE Trans. Computer-Aided Design, vol. 19, pp. 799-808, July 2000.

[83] D. Saraswat, R. Achar, and M. Nakhla, "Enforcing passivity for rational function based macromodels of tabulated data," in IEEE Topical Meeting on Electrical Performance of Electronic Packaging, pp. 295-298, Oct. 2003.

[84] A. Bellen, N. Guglilmi, and A. Ruehli, "Methods for linear systems of circuit delay differential equations of neutral type," IEEE Trans. Circuits Syst. I, vol. 46, no. 1, pp. 212-215, 1999.

[85] A. E. Ruehli, A. C. Cangellaris, and H.-M. Huang, "Three test problems for the comparison of lossy transmission line algorithms," in 11th IEEE Topical Meeting on Electrical Performance of Electronic Packaging, vol. 11, pp. 347-350, 2002.

[86] G. Lu, L. F. Yeung, D. W. C. Ho, and Y. Zhang, "Strict positive realness for linear time-invariant systems with time-delays," in Proc. IEEE Decision and Control Conf., pp. 5004-5009, Dec. 2000.

[87] E. Fridman and U. Shaked, “On delay-dependent passivity," IEEE Trans. Automatic Control, vol. 47, pp. 664-668, Apr. 2002. 
[88] A. Varga and P. Parrilo, "Fast algorithms for solving $H_{\infty}$-norm minimization problems," in Proc. IEEE Decision and Control Conf., vol. 1, pp. 261-266, Dec. 2001.

[89] M. Abramowitz and I. Stegun, Handbook of Mathematical Functions with Formulas, Graphs, and Mathematical Tables. 55, Washington, U.S. Govt. Print. Off., 1964.

[90] V. Mehrmann and D. Watkins, "Polynomial eigenvalue problems with Hamiltonian structure," Electronic Trans. on Numerical Analysis, vol. 13, pp. 106-118, 2002.

[91] F. Tisseur, "Structured pseudospectra for polynomial eigenvalue problems, with applications," SIAM J. Matrix Anal. Appl., vol. 23, no. 1, pp. 187-208, 2001.

[92] J. M. Ortega, Iterative Solution of Nonlinear Equations in Several Variables. Computer science and applied mathematics., New York: Academic Press, 1970.

[93] S. Niculescu, Delay Effects on Stability: A Robust Control Approach. Springerverlag, 2001.

[94] D. Breda, S. Maset, and R. Vermiglio, "Computing the characteristic roots for delay differential equations," IMA Journal of Numerical Analysis, vol. 24, pp. 1-19, 2004.

[95] K. Singh and Y. Ram, "Transcendental eigenvalue problem and its applications," vol. 40, pp. 1402-1407, 2002.

[96] N. Wagner and L. Gaul, "Eigenpath analysis of transcendental two-parameter eigenvalue problems," in European Congress on Computational Methods in Applied Sciences and Engineering, pp. 1-18, July 2004.

[97] K. S. Kundert, J. K. White, and A. Sangiovanni-Vincentelli, Steady-State Methods for Simulating Analog and Microwave Circuits. Boston: Kluwer Academic, 1990. 
[98] L. O.Chua and A. Ushida, "Algorithms for computing almost periodic steady-state response of nonlinear systems to multiple input frequencies," IEEE Trans. Circuits Syst., vol. 28, pp. 953-971, Oct. 1981.

[99] N. Borges de Carvalho and J. Pedro, "Novel artificial frequency mapping techniques for multi-tone simulation of mixers," in IEEE MTT-S Intl. Microwave Symp. Dig., vol. 1, pp. 455-458, 2001.

[100] K. Ogata, Modern control engineering. Upper Saddle River, N.J. : Prentice Hall, 3rd ed., 1997.

[101] G. Stewart and J. Sun, Matrix Perturbation Theory. Boston : Academic Press, 1990.

[102] K. Zhou and D. Doyle, Essentials of Robust Control. N.J.:Prentice Hall, 1998.

[103] K. Coperich, J. Morsey, A. Okhmatovski, V.I.and Cangellaris, and A. Ruehli, "Systematic development of transmission-line models for interconnects with frequencydependent losses," IEEE Trans. Microwave Theory Tech., vol. 49, pp. 1677-1685, Oct. 2001.

[104] J. Morsey and A. Cangellaris, "PRIME: passive realization of interconnect models from measured data," in IEEE Topical Meeting on Electrical Performance of Electronic Packaging, pp. 47-50, Oct. 2001.

[105] A. Dounavis, E. Gad, and M. Nakhla, "Passive model-reduction of multiport distributed networks including frequency dependent parameters," IEEE Trans. Microwave Theory Tech., pp. 2325-2334, Dec. 2000.

[106] O. Brune, "Synthesis of a finite two-terminal network whose driving-point impedance is a prescribed function of frequency," Journal of Mathematical Physics, vol. 10, pp. 191-236, 1931. 
[107] R. Bott and R. Duffin, "Impedance synthesis without use of transformers," Journal of Applied Physics, December 1948.

[108] J. Vlach, Computerized Approximation and Synthesis of Linear Networks. New York, Wiley, 1969.

[109] F. Y. Chang, "The generalized method of charactersitics fore waveform relaxation analysis of lossy coupled transmission lines," IEEE Trans. Microwave Theory Tech., vol. 37, pp. 2028-2038, Dec. 1989.

[110] D. Saraswat, R. Achar, and M. Nakhla, "A fast algorithm and practical considerations for passive macromodeling of measured/simulated data," IEEE Trans. Adv. Packag., vol. 27, pp. 57-70, Feb. 2004.

[111] A. Dounavis, "Time domain macromodels for high-speed interconnects.," Master's thesis, Carleton University, 2000.

[112] N. Nakhla, A. Ruehlit, M. Nakhla, R. Achar, and C. Chen, "Waveform relaxation techniques for simulation of coupled interconnects with frequency-dependent parameters," in IEEE Topical Meeting on Electrical Performance of Electronic Packaging, pp. 47-50, Oct 2005.

[113] A. Dounavis, X. Li, M. Nakhla, and R. Achar, "Passive closed-loop transmission line model for general purpose circuit simulators," IEEE Trans. Microwave Theory Tech., vol. 47, pp. 2450-2459, Dec. 1999.

[114] G. Shi and C.-J. Shi, "Model-order reduction by dominant subspace projection: Error bound, subspace computation, and circuit applications," IEEE Trans. Circuits Syst. I, vol. 52, pp. 975-993, May 2005. 
[115] L. Zhang and J. Lam, "Optimal weighted $l_{2}$ model reduction of delay systems," Int. J. Control, vol. 72, no. 1, pp. 39-48, 1999.

[116] S. Xu, J. Lam, S. Huang, and C. Yang, " $\mathrm{H}_{\infty}$ model reduction for linear time-delay systems: continuous-time case," Int. J. Control, vol. 74, no. 11, pp. 1062-1074, 2001.

[117] H. Baher, Synthesis of Electrical Networks. Chichester ; New York : Wiley, 1984.

[118] T. Palenius and J. Roos, “Comparison of reduced-order interconnect macromodels for time-domain simulation," IEEE Trans. Microwave Theory Tech., vol. 52, pp. 2240-2250, Sept 2004.

[119] W. Arnold and A. Laub, "Generalized eigenproblem algorithms and software for algebraic Riccati equations," Proceedings IEEE, vol. 72, no. 12, pp. 1746-1754, 1984. 